\title{
One- and Two-Dimensional Stirling Machine Simulation Using Experimentally Generated Reversing Flow Turbulence Models
}

Louis F. Goldberg

University of Minnesota

Minneapolis, Minnesota

August 1990

DISCLAIMER

Prepared for

Lewis Research Center

Under Grant NAG3-693

This report was prepared as an account of work sponsored by an agency of the United States This report any agency thereof, nor any Government. Neither the United States Government nor any agency lesal liability or responsibility for the accuracy, completeness, or usefulness of any information, apparatus, product, or process disclosed, or represents that its use would not infringe privately owned rights. Referprocess disclosed, or represents thercial product, process, or service by trade name, trademark, manufacturer, or otherwise does not necessarily constitute or imply its endorsement, recommendation, or favoring by the United States Government or any agency thereof. The views and opinions of authors expressed herein do not necessarily state or reflect those of the United States Government or any agency thereof.

\section{N/SA}

National Aeronautics and

Space Administration

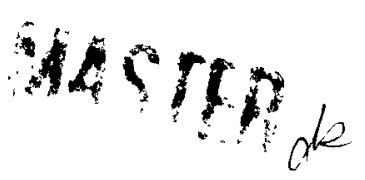




\section{DISCLAIMER}

This report was prepared as an account of work sponsored by an agency of the United States Government. Neither the United States Government nor any agency Thereof, nor any of their employees, makes any warranty, express or implied, or assumes any legal liability or responsibility for the accuracy, completeness, or usefulness of any information, apparatus, product, or process disclosed, or represents that its use would not infringe privately owned rights. Reference herein to any specific commercial product, process, or service by trade name, trademark, manufacturer, or otherwise does not necessarily constitute or imply its endorsement, recommendation, or favoring by the United States Government or any agency thereof. The views and opinions of authors expressed herein do not necessarily state or reflect those of the United States Government or any agency thereof. 


\section{DISCLAIMER}

Portions of this document may be illegible in electronic image products. Images are produced from the best available original document. 
NOTATION

CHAPTER 1: INTRODUCTION

1.1 Objectives

1.2 Project Evolution

1

1

2

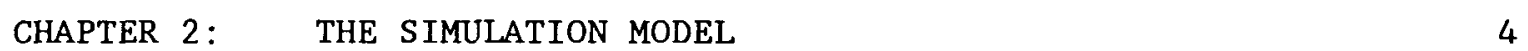

2.1 Introduction 4

2.2 The Integral Conservation Balances 4

2.3 The Turbulence Model 10

2.4 The Spatial Discretisation Scheme 15

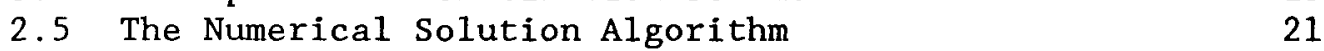

2.6 Information Propagation Models 23

$\begin{array}{lll}2.7 & \text { Boundary Advection } & 25\end{array}$

$\begin{array}{lll}2.8 \text { Closure } & 29\end{array}$

CHAPTER 3: SIMULATION OF THE SPACE POWER DEMONSTRATOR ENGINE 30

3.1 Introduction 30

3.2 Simulation Hardware 31

3.3 The SPDE System Model 33

3.4 Simulation Considerations $\quad 37$

3.5 Results $\quad 40$

3.6 Closure $\quad 56$

CHAPTER 4: COMPARISON AGAINST AN ANALYTIC SOLUTION 57

4.1 Introduction $\quad 57$

4.2 Problem Selection 58

4.3 Iberall's Analysis 59

4.4 The Simulation Mode1 60

$\begin{array}{ll}4.5 & \text { Applications }\end{array}$

$\begin{array}{lll}4.6 & \text { Interpretation } & 79\end{array}$

4.7 Numerical Accuracy Considerations 80

4.8 The Crank-Nicholson Integration Scheme 80

4.9 Results $\quad 81$

4.10 Conclusion $\quad 95$

$\begin{array}{ll}4.11 \text { Prognosis } & 96\end{array}$ 
CHAPTER 5: SIMULATION OF THE MECHANICAL ENGINEERING TEST RIG 97

$\begin{array}{lll}5.1 & \text { Introduction } & 97\end{array}$

$\begin{array}{ll}5.2 & \text { Description of the Test Rig } 98\end{array}$

$\begin{array}{ll}5.3 \text { Baseline Case Simulation Application } & 101\end{array}$

$\begin{array}{lll}5.4 & \text { Baseline Case Results } & 102\end{array}$

5.5 Comparison Case Simulation Application $\quad 144$

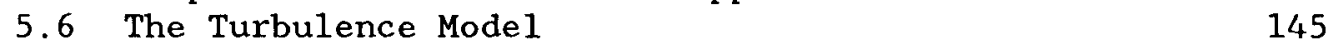

5.7 Comparison Case Experimental Data 166

$\begin{array}{lll}5.8 & \text { Comparison Case Results } & 167\end{array}$

5.9 Conclusion $\quad 184$

$\begin{array}{lll}\text { CHAPTER 6: } & \text { CONCLUSION } & 185\end{array}$

$\begin{array}{lr}\text { REFERENCES } & 187\end{array}$ 
ROMAN

\section{Italicised Lower Case}

$\begin{array}{ll}a & \text { constant } \\ b & \text { constant } \\ d & \text { diameter } \\ f & \text { frequency } \\ g & \text { scalar mass flux } \\ k & \text { turbulence kinetic energy } \\ 1 & \text { length } \\ m & \text { index limit } \\ q & \text { contact heat flux scalar } \\ r & \text { radius } \\ t & \text { time } \\ v & \text { velocity scalar } \\ \mathbf{x} & \text { displacement scalar or variable } \\ y & \text { variable }\end{array}$

\section{Italicised Upper Case}

$\begin{array}{ll}A & \text { area } \\ C & \text { heat capacity } \\ E & \text { external and mutual energy } \\ G & \text { total flux } \\ H & \text { enthalpy } \\ K & \text { constant } \\ M & \text { mass } \\ N & \text { non-dimensional parameter } \\ P & \text { pressure } \\ R & \text { gas constant } \\ T & \text { temperature } \\ U & \text { internal energy } \\ V & \text { volume }\end{array}$




\section{Bold Lower Case}

f external and mutual force

8 mass flux density

n unit outward normal

q contact heat flux

s contact force

$\mathbf{v}$ velocity

x displacement

\section{Bold Upper Case}

$\begin{array}{ll}\text { D } & \text { deformation tensor } \\ \text { I } & \text { identity matrix } \\ \text { T } & \text { extra stress tensor }\end{array}$

\section{Computer Programme Variables}

NHL index of last heater mass/energy discrete volume

NKF index of first cooler mass/energy discrete volume

NRF index of first regenerator mass/energy discrete volume

NRL index of last regenerator mass/energy discrete volume

\section{GREEK}

1. Upper Case

$\begin{array}{ll}\Lambda & \text { bulk compressibility } \\ \Phi & \text { dissipation }\end{array}$

2. Lower Case

$\begin{array}{ll}\alpha & \text { constant } \\ \beta & \text { constant } \\ \delta & \text { unit vector component } \\ \epsilon & \text { turbulence kinetic energy dissipation rate }\end{array}$




\section{HEBREW}

$*$

OPERATORS

$\mathrm{d}$

dd $t$

D

$f()$

$\lambda()$

o

$\Delta$

$\nabla$

$\int$

$\Sigma$

$\bar{\psi}=[\bar{t}]^{\psi} \psi$

[v] $\bar{\psi}$

[tv] $\bar{\psi}$

$\bar{\psi}={ }_{[t t]} \bar{\psi}$

$|\psi|$

-

:

ก

$u$

$c$ dynamic viscosity

kinematic viscosity

density

extra shear stress tensor component

generalised scalar, vector, or tensor quantity

specific dissipation rate

transformation tensor

total derivative

total derivative with respect to time

substantive derivative

function of

quantisation function

partial derivative

incremental change

divergence

integral

summation

time average of $\psi$

volume average of $\psi$

time average of volume average of $\psi$

time average of time average of $\psi$

absolute value or magnitude of $\psi$

scalar product of vectors, vector product of vector and tensor

scalar product of tensors

intersection

union

proper subset 


\section{$\underline{\text { SUBSCRIPTS }}$}

$\begin{array}{ll}a & \text { acoustic } \\ c h & \text { characteristic } \\ i & \text { index } \\ I & \text { index } \\ j & \text { index } \\ k & \text { index } \\ M & \text { index limit in two-dimensional space } \\ (m) & \text { material body } \\ n & \text { momentum discrete volume } \\ N & \text { index limit in two-dimensional space } \\ n L & \text { momentum discrete volume, left hand } \\ n r & \text { radial momentum discrete volume } \\ n R & \text { momentum discrete volume, right hand } \\ n x & \text { axial momentum discrete volume } \\ P & \text { at constant pressure } \\ P e & \text { Peclet } \\ P r & \text { Prandtl } \\ r & \text { regenerator } \\ R e & \text { Reynolds } \\ (s) & \text { system of particles } \\ T & \text { at constant temperature } \\ V a & \text { Valensi } \\ x & \text { axial } \\ \Delta t & \text { time increment }\end{array}$

\section{SUPERSCRIPTS}

$\begin{array}{ll}\text { (t) } & \text { turbulent } \\ \mathrm{T} & \text { transpose } \\ \mathrm{s} & \text { previous time step } \\ * & \text { distinguishing indicator } \\ \text {. } & \text { fluctuating component } \\ \text {. } & \text { per unit mass } \\ & \text { time rate of change }\end{array}$


The activities described in this report do not constitute a continuum but rather a series of linked smaller investigations in the general area of one- and two-dimensional stirling machine simulation. The initial impetus for these investigations was the development and construction of the Mechanical Engineering Test Rig (METR) under a grant awarded by NASA to Dr Terry Simon at the Department of Mechanical Engineering, University of Minnesota. The purpose of the METR is to provide experimental data on oscillating turbulent flows in Stirling machine working fluid flow path components (heater, cooler, regenerator, etc.) with particular emphasis on laminar/turbulent flow transitions.

Hence, the initial goals for the grant awarded by NASA were, broadly, to provide computer simulation backup for the design of the METR and to ana1yze the results produced. This was envisaged in two phases: first, to apply an existing one-dimensional stirling machine simulation code to the METR and second, to adapt a two-dimensional fluid mechanics code which had been developed for simulating high Rayleigh number buoyant cavity flows to the METR. The key aspect of this latter component was the development of an appropriate turbulence model suitable for generalised application to Stirling simulation. A final step was then to apply the two-dimensional code to an existing Stirling machine for which adequate experimental data exist.

The work described herein was carried out over a period of three years on a part-time basis. Forty percent of the first year's funding was provided as a match to the NASA funds by the Underground Space Center, University of Minnesota, which also made its computing facilities available to the project at no charge.

\subsection{OBJECTIVES}

With the advantage of a posteriori clarity, the following overall objectives guided the course of the work:

1. Apply an existing one-dimensional simulation code to the METR.

2. Adapt and apply an existing two-dimensional fluid mechanics code to the METR.

3. Use the METR experimental results to guide the development of a turbulence model appropriate for generalised application to Stirling machine simulation. 
4. Validate the two-dimensional simulation including its turbulence model against experimental data for an existing stirling engine.

\subsection{PROJECT EVOLUTION}

The basic simulation codes applied to the METR are the fully implicit, discrete volume simulations developed during the course of the author's PhD research program (Go87). The application of the one-dimensional version of the code to the METR was elementary and provided some design guidance to Simon and Seume (the graduate student conducting the METR research) in developing the final design of the rig.

Because a significant delay prior to the commissioning of the rig was expected, it was decided to proceed with applying the one- and two-dimensional simulations to a Stirling engine, the latter simulation initially without the inclusion of a turbulence model. NASA chose the Space Power Demonstrator Engine (SPDE) as the target engine. This back-to-back, free-piston design is characterised by an operating frequency of $100 \mathrm{~Hz}$ and a mean. pressurisation of 150 bars which, combined with a relatively short working fluid flow path, yield an engine characteristic number $\left(N_{c h}\right)$ (see section 2.6) of about 25. This means that there are roughly 25 complete information propagation traverses between the expansion and compression space pistons during each cycle. This may be compared with a typical characteristic number of 96 for the GM-GPU3 kinematic engine. In the case of the GM-GPU3 engine, the characteristic number proved to be large enough so that modeling of information propagation effects did not prove necessary in order to match the experimental data (Go87). However, in the case of the SPDE, such modelling of information propagation did enable agreement between the measured and simulated piston indicated works to be obtained.

This elicited some controversy not only within NASA but also among other Stirling engine analysts who expressed doubt whether information propagation effects are physically relevant at low Mach numbers. Hence, a significant deviation of the grant was initiated into an investigation of the information propagation phenomenon in the context of a physical application remote from Stirling engines. While not settling the controversy, this investigation did reveal the limitations of the simulation code by establishing a lower characteristic number limit below which the code was judged inapplicable.

Within these limits, the two-dimensional fluid dynamics code was modified and successfully applied to the SPDE. The methodology adopted was to treat the heater as a two-dimensional entity represented by a single "typical" tube in an otherwise one-dimensional system. This was intended to facilitate the application of the METR turbulence data to an actual engine (via the simulation code) since the rig would be configured to represent just such an SPDE heater tube. A significant aspect of this application was the development of a mesh generation scheme enabling a "seamless" junction between the one-dimensional rectilinear and two-dimensional cylindrical spatial discretisations. 
At this stage, the METR still did not yield quantitative data suitable for turbulence model development. Nevertheless, the two-dimensional SPDE code was ported to the METR geometry, albeit without the inclusion of a turbulence model. Some mean velocity qualification comparisons were made between the simulated and preliminary experimental data. These comparisons revealed systematic errors in the experimental data. The errors included flow anomalies (apparently caused by piston/cylinder "sticking"), the absence of ambient boundary conditions and hot-wire anemometer calibration uncertainties. Qualitatively useful data did however become available when Joerge Seume published his $\mathrm{PhD}$ thesis (Se88). Hence, since the grant period was drawing to a close, development of the turbulence model was initiated without the benefit of an experimental benchmark. Finally, a single half-cycle of quantitatively useful turbulence data (despite the continued existence of systematic errors) for the SPDE heater tube configuration was eventually delivered about three weeks prior to the termination of the grant. This allowed barely enough time to make some preliminary turbulence model evaluations and to define the critical issues in oscillating flow turbulence modelling. The larger objective of applying the turbulence model to the SPDE could not be fulfilled.

The structure of this report thus reflects the modus operandi of the grant itself, comprising a compendium of sectional reports delivered to NASA at the termination of each phase. Chapter 2 summarizes the simulation model and its theoretical foundations; chapter 3 discusses the simulation of the SPDE; chapter 4 describes an investigation of the information propagation issue based upon an analytic description of a transmission line; and chapter 5 is devoted to the simulation of the METR and the development of a turbulence model. Salient conclusions and some directions for future research arising therefrom are presented chapter 6 . 


\section{$2.1 \quad$ INTRODUCTION}

This chapter presents a narrative overview of the physics and numerics of the simulation model. Included is the postulational basis from which the conservation equations are derived as well as the definitions of the discretisation, turbulence and information propagation models. Since the symbolic development of the simulation model is voluminous, the reader is directed toward reference Go87 for a complete and rigorous derivation of all the equations presented.

The philosophical basis upon which the simulation model rests is described by Tisza (Ti66) as the 'postulational' approach in his discussion of the evolution of the concepts of thermodynamics. In summarizing the efficacy of the postulational approach, Tisza makes the following critical observation:

'First, and most important, we claim no absolute validity for our postulational basis. The validity of the postulates and the usefulness of the primitive concepts are only tentative and have to be justified by the experimental verification of the implications of the theory.'

Thus the postulational approach used to develop a symbolic description of the fluid dynamics of Stirling cycle machines ultimately can be justified only by the extent to which the results produced can be given validity by experimental observation.

\subsection{THE INTEGRAL CONSERVATION BALANCES}

The integral conservation balances forming the backbone of the simulation codes are derived from four postulates. The first postulate is based on the classical concept that matter is uniformly distributed through space. Even though this postulate is known to be unrealistic in terms of the quantised, discontinuous nature of matter, its usefulness lies in the simplicity with which macroscopic phenomena may be described. The following statement of the first postulate is adopted:

Postulate I Matter is continuous and distributed uniformly within an arbitrary bounded space.

This statement is more restrictive than those usually offered ( 181 , ZH76) since the uniform and continuous distribution of matter is postulated only within a space delineated by boundaries which is defined herein as a discrete volume. Thus a discrete volume admits the existence of discontinuities at its boundaries. This means that physical phenomena such as 
shock waves and numerical phenomena such as volume-averaged property discontinuities are accommodated within the piecewise continuum model postulated.

Having thus defined iscrete volume, the essential requirement is to describe the temporal variat of intensive properties within the discrete volume from macroscopically ciss "vable conditions.

For a generalised scalar, ctor or tensor quantity $\psi$ defined by:

$\psi=\psi(\mathbf{x}, t)$

the total temporal derivative of $\psi$ for a cohesive material body is given by:

$\operatorname{dd} t \int_{V_{(m)}} \psi \mathrm{d} V=\int_{V_{(m)}}(\partial \psi / \partial t) \mathrm{d} V+\int_{A_{(m)}} \psi(\mathrm{v} \cdot \mathrm{n}) \mathrm{d} A$

Equation (2.2) indicates that the total change of $\psi$ for the entire material body is a function of the change of $\psi$ at each fixed point within the body plus the transport of $\psi$ at the boundaries of the body. This equation, which is known as the 'transport theorem' (S181) places no restrictions on the nature of the body other than it be regarded as an autonomous entity within a given discrete volume $V_{(m)}$ and that it have the characteristics of a continuum. In particular, the degree of cohesiveness of the body is arbitrary, so that generalisation to a system of particles of arbitrary cohesiveness (that is, liquids, solids or gases) yields:

$\operatorname{dd} t \int_{V_{(S)}} \psi \mathrm{d} V=\int_{V_{(S)}}(\partial \psi / \partial t) \mathrm{d} V+\int_{A_{(S)}} \psi\left(v_{(s)} \cdot \mathrm{n}\right) \mathrm{d} A$

Equation (2.3) is known as the 'generalised transport theorem' (S181) and, in essence, is the symbolic realisation of the first postulate.

The transport theorem of equation (2.2) provides the means by which macroscopic conservation postulates may first be transformed into their microscopic or differential counterparts that apply within the discrete volume. Thereafter, the generalised transport theorem permits the differential conservation balances to be applied to a system of particles such as that comprising the working fluid of a stirling cycle machine.

The conservation postulates are expressed strictly in terms of macroscopic phenomena. Hence the macroscopic conservation of mass for an arbitrary material body is expressed by the following postulate: 
Postulate II The mass of an autonomous material body is independent of time.

Symbolically, this may be expressed as:

$\operatorname{dd} t \int_{V_{(m)}} \rho \mathrm{d} V=0$

Choosing $\psi=\rho$ (that is, mass per unit volume or density is the transport property) in equation (2.2) produces the differential conservation balance:

$\partial \rho / \partial t=-(\nabla \cdot \rho \mathbf{v})$

in a Eulerian frame of reference.

The integral mass balance applicable to a discrete volume is obtained from equations (2.5) and (2.3) and is given by:

$\mathrm{d} M_{(s)} / \mathrm{d} t=\int_{A_{(s)}} \rho\left\{\left(\mathrm{v}-\mathrm{v}_{(s)}\right) \cdot-\mathrm{n}\right\} \mathrm{d} A$

In this generalised or combined Eulerian/Lagrangian form, the rate of change of mass of a system of particles is equal to the net advection of mass across the boundaries of the system. The advection velocity is the relative velocity between the particles and the boundary itself.

A statement of the macroscopic conservation of momentum for an arbitrary material body expresses the third postulate, which is generally referred to as Euler's first law (S181):

Postulate III The time rate of change of the momentum of an autonomous material body relative to the fixed stars is equal to the sum of the forces acting on the body.

Postulate III may be expressed symbolically as:

$\operatorname{dd} t \int_{V_{(m)}} \rho \mathbf{v d V}=\int_{A_{(m)}} \mathbf{s d A}+\int_{V_{(m)}} \rho \hat{\mathrm{f}} \mathrm{d} V$ 
where $s$ denotes the contact forces per unit area and $f$ denotes the external and mutual forces.

Selecting $\psi=\rho v$ (the momentum per unit volume) in the transport theorem (equation (2.2)), allows the following Eulerian differential momentum conservation equation to be derived:

$\partial(\rho \mathbf{v}) / \partial t+\nabla \cdot(\rho \mathbf{v v})=\nabla \cdot \mathbf{T}-\nabla P+\rho \hat{\mathbf{f}}$

It should be noted that the sign convention adopted for the extra shear stress tensor $T$ is such that $T$ represents the stress acting at any point within a material body. Modifying Stokes' hypothesis (Sc79) for gaseous fluids by including a 'bulk viscosity' (BS60) allows $T$ to be expressed by:

$\mathbf{T}=\mu\left\{\nabla \mathbf{v}+(\nabla \mathbf{v})^{\mathrm{T}}\right\}+\{(\Lambda-2 \mu / 3)(\nabla \cdot \mathbf{v})\} \mathrm{I}$

Together, equations (2.8) and (2.9) represent the Navier-Stokes equations, which are usually independently derived on a more intuitive basis ( $\mathrm{Sc} 79$, BS60).

The generalised combined Eulerian/Lagrangian form of the integral momentum conservation balance is obtained by substituting equation (2.8) into equation (2.3) which produces:

$$
\begin{aligned}
\mathrm{d}\left({ }_{[\mathrm{v}]} \overline{\mathrm{v}} M_{(S)}\right) / \mathrm{d} t= & \int_{A_{(S)}} \rho \mathrm{v}\left\{\left(\mathrm{v}-\mathrm{v}_{(S)}\right) \cdot-\mathrm{n}\right\} \mathrm{d} A-\int_{A_{(S)}} P \mathrm{nd} A-\int_{A_{(S)}}(\mathrm{T} \cdot-\mathbf{n}) \mathrm{d} A \\
& +\int_{V_{(S)}} \rho \hat{\mathrm{f}} \mathrm{d} V
\end{aligned}
$$

Thus the rate of change of momentum of a system of particles is equal to the net advection of momentum across the boundaries of the system relative to the system boundary velocity plus the contact, mutual, and external forces acting on the system.

The fourth postulate is defined by the conservation of energy for a material body. In the context of a discrete volume analysis, the first law of thermodynamics which is adopted by most authors as their postulational basis (Sc79, for example) is not specific enough for a macroscopic material body (Go87). Thus the following formulation advocated by slattery (S181) is preferred: 
The time rate of change of the internal and kinetic energy of an autonomous material body relative to the fixed stars is the sum of the rate at which forces acting on the body do work on the body and the rate of energy transmission to the body.

Symbolically, this may be expressed as:

$\mathrm{dd} t \int_{V_{(m)}} \rho\left(\hat{U}+\mathrm{v}^{2} / 2\right) \mathrm{d} V=\int_{A_{(m)}}(\mathrm{v} \cdot \mathbf{s}) \mathrm{d} A+\int_{V_{(m)}} \rho(\mathrm{v} \cdot \hat{\mathrm{f}}) \mathrm{d} V+\int_{A_{(m)}} \dot{q} \mathrm{~d} A+\int_{V_{(m)}} \dot{\hat{i} E \mathrm{~d} V}$

where $s$ and $f$ are defined for equation (2.7), $\dot{q}$ denotes the contact energy transmission rate per unit area, and $\hat{E}$ denotes the external and mutual energy transmission rate. The first and second terms on the right hand side represent the work done by the corresponding force terms in equation (2.7)

Choosing the internal plus kinetic energy per unit volume as the transport property by setting $\left(\psi=\rho\left(U+v^{2} / 2\right)\right.$ ) in the transport theorem (equation (2.2)), the following Lagrangian differential energy equation may be derived (Go87):

$\rho D\left(\hat{U}+\mathrm{v}^{2} / 2\right) / D t=\rho((\mathbf{v} \cdot \hat{\mathbf{f}})+\dot{E})+\nabla \cdot(\mathrm{T} \cdot \mathbf{v})-\nabla \cdot(P \mathbf{v})-\nabla \cdot \dot{\mathbf{q}}$

This equation describes the differential conservation of thermal and mechanical energy. It can be simplified by observing that the differential conservation of mechanical energy may be determined separately using postulate III. Forming the scalar product of the Lagrangian form of equation (2.8) with $v$ yields the differential conservation of mechanical energy equation in a Lagrangian frame of reference:

$\rho D\left(v^{2} / 2\right) / D t=\nabla \cdot(T \cdot v)-(T: \nabla v)-(\mathbf{v} \cdot \nabla P)+\rho(\mathbf{v} \cdot \hat{\mathbf{f}})$

The second term on the right-hand side is a tensor scalar product which represents the irreversible conversion of mechanical energy into thermal energy, or dissipation. Subtracting equation (2.13) from equation (2.12) and expressing the result in Eulerian terms produces:

$\partial(\rho \hat{U}) \partial t+\nabla \cdot(\rho \hat{U} \mathbf{v})=\rho \dot{\hat{E}}+(\mathbf{T}: \nabla \mathbf{v})-P(\nabla \cdot \mathbf{v})-\nabla \cdot \dot{\mathbf{q}}$

This equation defines the differential conservation of thermal energy. It may be noted that equations (2.12) and (2.8) contain a redundancy that is absent from equations (2.14) and (2.8). Although either set is admissible, and both 
sets must ultimately yield identical results, the latter set is preferred because of the computational simplicity and convenience it affords in describing stirling machine fluid dynamics.

Choosing the internal energy per unit volume as the transport property in the generalized transport theorem (that is, $\psi=\rho U$ in equation (2.3)) and substituting equation (2.14) produces the general Eulerian/Lagrangian integral form:

$$
\begin{aligned}
\mathrm{d}\left({ }_{[\mathrm{v}]} \overline{\hat{U}} M_{(s)}\right) / \mathrm{d} t= & \int_{V_{(s)}}\{\dot{\hat{E}}+(\mathrm{T}: \nabla \mathrm{v})+(\mathrm{v} \cdot \nabla P)\} \mathrm{d} V+\int_{A_{(s)}}(\dot{\mathrm{q}} \cdot-\mathrm{n}) \mathrm{d} A \\
& +\int_{A_{(S)}} \rho \hat{H}\left\{\left(\mathrm{v}-\mathrm{v}_{(s)}\right) \cdot-\mathrm{n}\right\} \mathrm{d} A-\int_{A_{(S)}} P\left(\mathrm{v}_{(s)} \cdot-\mathrm{n}\right) \mathrm{d} A
\end{aligned}
$$

Thus the rate of change of internal energy of a system of particles is equal to the sum of the following components, which in left to right sequence are:

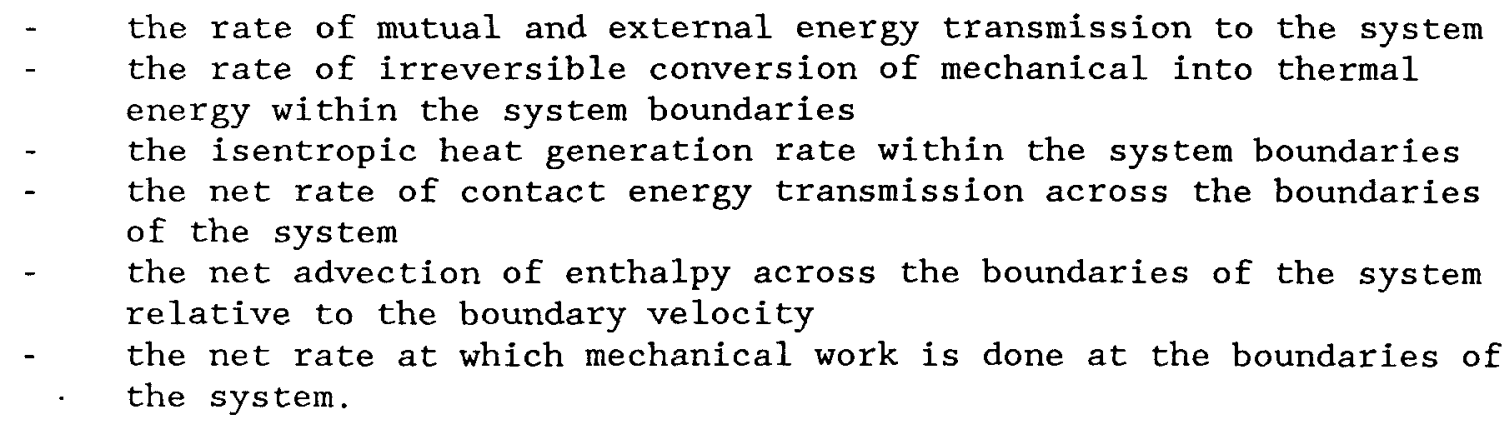

In order to implement the integral balances for gaseous fluids, an equation of state is required. In keeping with the established practice for Stirling machine analysis (Sc1871, Wa73), the ideal gas equation of state is used here, namely:

$P V=M R T$

Nevertheless, there are no intrinsic restrictions placed on the form of the equation of state; other equations describing the behavior of real gases, such as that of Redlich and Kwong (RK49), may be used. Owing to their relative complexity, however, such equations are not as numerically convenient as the ideal gas equation.

Equations (2.6), (2.10), (2.15), and (2.16) thus provide the analytic basis in terms of a discrete volume continuum model for determining the working fluid behavior of stirling cycle machines. 


\subsection{THE TURBULENCE MODEL}

The integral balances of equations (2.6), (2.10), and (2.15) are strictly applicable in the limit as $\Delta t \rightarrow 0$ (Hi75). However, when the balances are applied to systems in which $\Delta t$ is finite, then the balances are precise only for laminar flow conditions. Under turbulent flow conditions, the transport properties $\psi$ may experience random fluctuations with periods less than $\Delta t$, thus invalidating the instantaneous constancy of the temporal gradients implied by the equations as described. The instantaneous value of $\psi$ may be represented as the sum of a time-averaged component and a fluctuating component:

$\psi=\bar{\psi}+\psi^{\prime}$

Attention here is focussed on obtaining the time-averaged quantities $\bar{\psi}$ directly since the computational effort necessary to obtain $\psi$ is currently beyond the scope of practical stirling machine simulation. The most general approach to obtaining the time-averaged or turbulent transport balances is to perform the averaging process on the integral balances directly (S181). This admits fluctuating discrete volume geometries such as those occurring in Lagrangian systems. Time-averaging equations (2.6), (2.10), and (2.15) results in:

Mass :

$\mathrm{d}_{(s)} / \mathrm{d} t=\int_{A_{(s)} \rho\left\{\left(\mathrm{v}-\mathrm{v}_{(s)}\right) \cdot-\mathrm{n}\right) \mathrm{d} A}$

Momentum:

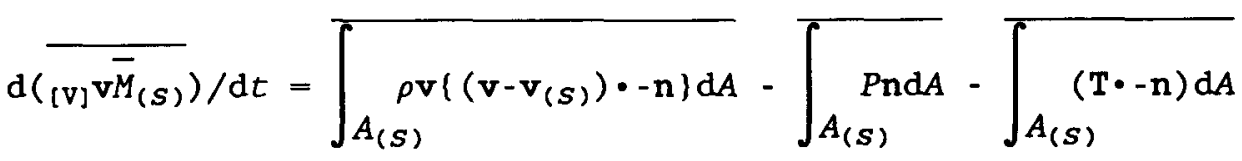

$$
\begin{aligned}
& -\int_{V(s)} \hat{\rho f d} V
\end{aligned}
$$


Energy:

$$
\begin{aligned}
\mathrm{d}\left(\overline{[\mathrm{v}]} \overline{\hat{\hat{U}} M_{(S)}}\right) / \mathrm{d} t= & \int_{V_{(S)}(\rho \dot{\hat{E}}+(\mathrm{T}: \nabla \mathrm{v})+(\mathrm{v} \cdot \nabla P) \mathrm{d} V}-\overline{\int_{A_{(S)}}(\dot{\mathrm{q}} \cdot-\mathrm{n}) \mathrm{d} A} \\
& +\overline{\int_{A_{(s)} \rho \hat{H}\left(\left(\mathrm{v}-\mathrm{v}_{(s)}\right) \cdot-\mathrm{n}\right\} \mathrm{d} A}}-\overline{\int_{A_{(s)}} P\left(\mathrm{v}_{(s)} \cdot-\mathrm{n}\right) \mathrm{d} A}
\end{aligned}
$$

Equations (2.18) through (2.20) are by definition also applicable under laminar flow conditions, since from equation $(2.17) \psi=\bar{\psi}$ when $\psi^{\prime}=0$.

The principal difficulty in solving the time-averaged integral balances is the unavailability of the fluctuating component of the transport properties. In order to obtain the time-averaged properties directly, the analytic approach adopted for dealing with the unknown fluctuating components falls into the category of 'Reynolds averaged equations' ( $\mathrm{Fe} 83$ ). In this context, since the integral balances derived include both time- and volumeaveraging, Ferziger makes the following observation:

'... The equations describing the mean field contain averages of products of fluctuating velocities and there are fewer equations than unknowns - the well-known closure problem. In fact, the set of equations can never be closed by further averaging; a closure assumption, or what is the same thing, a turbulence model, has to be introduced. The closure assumption must represent the unknown higherorder average quantities in terms of lower-order quantities that are computed explicitly.'

The minimum set of assumptions constituting the turbulence model adopted here is stated in terms of the six restrictions discussed below. These restrictions enable a time-averaged solution of the integral balances to be obtained with reasonable computing resources. It must be emphasized, however, that the turbulence model adopted is not definitive and is subject to amendment by experimental data.

\section{Restriction I}

The turbulent flow is stationary such that:

$\Delta t_{\text {turbulence characteristic }} \ll \Delta t_{\text {analytic }}$ time increment

\section{Restriction II}

The turbulent flow field is spatially homogeneous such that: 
$\Delta V_{\text {turbulence characteristic }} \ll \Delta V_{\text {discrete volume }}$

Rigorously, equations (2.21) and (2.22) are conflicting conditions for any turbulent flow because if such a flow field is homogeneous, then it is simultaneously a decaying flow field. However, if it is also stationary, then the dissipation in the field can only be balanced by a non-homogeneity in order to maintain the decaying characteristic. The following rationale offered by Hinze (Hi75) is adopted here for proceeding with the stationary, homogeneous flow field model:

'... Fortunately, the rate of decay of the mean properties is rather slow with respect to the time scale of the smaller eddies. Therefore, the actual state of non-stationarity is considered not to be a serious drawback in the experimental study of the smaller scale turbulence. For the theoretical study, this makes it possible to apply the concepts and theories of stationary random processes.'

\section{Restriction III}

The stationarity of the turbulent flow field is sufficient for the equality of the first and second order time averages, or equation (2.21) implies that:

$$
\bar{\psi}=\bar{\psi}
$$

Hence by taking the time average of equation (2.17) it immediately follows that:

$\overline{\psi^{\prime}}=0$

\section{Restriction IV}

The ergodic hypothesis is valid for scalar turbulent fields.

The ergodic hypothesis states that for a stationary and homogeneous turbulence:

$[\mathrm{v}] \bar{\psi}^{\star}=\bar{\psi}^{\star}={ }_{[\text {ensemble }]} \bar{\psi}^{\star}$

where $\psi^{*}$ represents a scalar or component of a vector. Hence from restriction IV and equa -ions (2.23) through (2.25) it follows that:

$[\mathrm{v}]_{\text {scalar }}^{\prime}=0$

Hence, in particular, for density and temperature: 
$\left[\mathrm{v} \bar{\rho}^{\prime}={ }_{[\mathrm{v}]} \bar{T}^{\prime}=0\right.$

Equations (2.27) constitute two of the explicit restrictions placed on the simulation by the turbulence model, namely, that the temporal fluctuation of the volume-averaged density and temperature are zero under the restrictions of equations (2.21) and (2.22). Since the ergodic hypothesis is applied to scalar properties only and equation (2.26) is limited to volume averages, nonzero fluctuations of volume-averaged vector fields and correlations involving non-volume-averaged scalar fields are permissible. This requires another restriction, namely:

\section{Restriction V}

The effect on the mean flow resultant from vector turbulent fields may be modelled.

In particular, the effect of the turbulent velocity field fluctuations on the time-averaged flow field may be determined using a model such as an empirical correlation (for example, that between friction factor and Reynolds number) or a two-parameter turbulence model of the $k-\epsilon$ variety.

The last restriction is defined by:

\section{Restriction VI}

The discrete volume boundaries do not experience temporal fluctuations. This may be expressed symbolically as:

$$
A^{\prime}(s)=0
$$

which in turn implies that:

$$
V^{\prime}(s)=0 \text { and } \overline{\psi \cdot n}=\bar{\psi} \cdot \mathbf{n}
$$

Equations (2.28) do not place a restriction on the Lagrangian condition $v=v_{(s)}$ as suggested by equation (2.3). Under turbulent conditions, this is achieved by setting $v_{(s)}=\bar{v}$ and admitting a turbulent flux as a function of $v-v_{(s)}$ (which is equal to $v^{\prime}$ ) across the Lagrangian boundary. In effect this converts a turbulent Lagrangian boundary into a combined Eulerian/Lagrangian boundary. In the context of Stirling machine numerical analysis, restriction VI enables generality to be maintained without the necessity of allowing turbulent discrete volume boundary movement, enabling a significant simplification of the subsequent analysis. 
At this stage, it is convenient to introduce the mass flux $g$ into the equations as a means of simplifying the numerical model. $g$ is defined by:

$\mathbf{g}=\rho \mathbf{v}$

Applying equation (2.29) and the six restrictions of the turbulence model to the time-averaged conservation balances of equations (2.18) to (2.20) yields (in combined Eulerian/Lagrangian form):

Mass:

$\mathrm{d} \bar{M}_{(s)} / \mathrm{d} t=\int \overline{\bar{A}}_{(s)}\left\{\left(\overline{\mathrm{g}}-\overline{\rho \mathrm{v}}_{(s)}\right) \cdot-\mathrm{n}\right\} \mathrm{d} \bar{A}$

Momentum:

$$
\begin{aligned}
\mathrm{d}\left({ }_{[\mathrm{tV}]} \overline{\mathrm{g}}_{\overline{\mathrm{V}}} \bar{V}_{(s)}\right) / \mathrm{d} t= & \left.\int_{\bar{A}_{(S)}} \overline{\mathrm{g}}_{(S)}\left(\overline{\mathrm{v}}-\overline{\mathrm{v}}_{(s)}\right) \cdot-\mathrm{n}\right\} \mathrm{d} \bar{A}-\int_{\bar{A}_{(s)}} \bar{P}_{\mathrm{nd} \bar{A}} \\
& -\int_{\bar{A}_{(s)}}\left\{\left(\overline{\mathrm{T}}+\mathrm{T}^{(\mathrm{t})}\right) \cdot-\mathrm{n}\right\} \mathrm{d} \bar{A}+\int_{\bar{V}_{(s)}} \overline{\hat{\mathrm{f}} \mathrm{d} \bar{V}}
\end{aligned}
$$

Energy:

$$
\begin{aligned}
& \left.\hat{C}_{V} \mathrm{~d}\left({ }_{[\mathrm{tV}]} \bar{T} \bar{M}_{(s)}\right) / \mathrm{d} t=\int_{\bar{V}(s)} \frac{\overline{\hat{i}}}{(\rho E}+(\overline{\mathrm{T}: \nabla \mathrm{v}})+(\overline{\mathrm{v} \cdot \nabla P})\right) \mathrm{d} \bar{V}+\int_{\bar{A}_{(s)}}\left(\left(\overline{\dot{\mathrm{q}}}+\mathrm{q}^{(t)}\right) \cdot-\mathrm{n}\right) \mathrm{d} \bar{A} \\
& -\int_{\bar{A}_{(s)}}\left(\overline{P \mathbf{v}}_{(s)} \cdot \mathbf{n}\right) \mathrm{d} \bar{A}+\hat{C}_{P} \int_{\bar{A}_{(s)}}\left(\bar{T}\left(\overline{\mathbf{g}}^{-\rho \mathbf{v}_{(s)}}\right) \cdot-\mathbf{n}\right) \mathrm{d} \bar{A}
\end{aligned}
$$

Expressing the equation of state (equation (2.16)) in volume-averaged terms and applying the turbulence model yields:

$$
{ }_{[\mathrm{tv}]} \bar{P}={ }_{[\mathrm{tv}]} \bar{\rho} R_{[\mathrm{tv}]} \bar{T}
$$

The Reynolds stress tensor $T^{(t)}$ in equation (2.31) is given by:

$$
T^{(t)}=\left(\overline{\mathbf{g}} \overline{\mathrm{v}}-\overline{\mathrm{g}} \overline{\mathrm{v}}_{(s)}\right)-\left(\overline{\mathrm{g} v}-\overline{\mathrm{gv}}_{(s)}\right)
$$


Analogously, the turbulent enthalpy flux vector $q^{(t)}$ in equation (2.32) is defined by:

$\left.\mathrm{q}^{(t)}=\hat{C}_{P}\left(\overline{(\mathbf{g T}}-\overline{\rho \mathbf{v}_{(s)} \mathrm{T}}\right)-\left(\overline{\mathrm{g}} \bar{T}-\overline{\rho \mathbf{v}}_{(s)} \bar{T}\right)\right\}$

Equations (2.34) and (2.35) cannot be solved since they contain additional unknowns for which there are no additional equations (the closure problem). Hence a turbulence model is required to solve these equations.

\subsection{THE SPATIAL DISCRETISATION SCHEME}

In his comprehensive review of computational fluid mechanics, Roache (Ro82) describes a variety of spatial and temporal discretisation schemes for the numerical application of the differential conservation balances which may be loosely categorised into 'coincident' and 'staggered' mesh systems. These mesh systems may be applied both spatially and temporally, thus yielding a multiplicity of schemes involving coincident and staggered mesh systems.

In a coincident mesh scheme, the three differential transport properties--density, velocity, and temperature--are evaluated at the same time and/or at the same spatial location. However, in a spatially staggered mesh, generally the density and temperature are computed at one set of grid points while the velocities are computed at an offset grid point mesh. In a temporally staggered mesh, the velocity is computed at a half time step offset from the density and temperature. In recent years, a de facto consensus has emerged that spatially staggered, temporally coincident discretisation schemes are convenient and useful for fluid flow modelling (Fe83, Pa80). Roache infers that the first use of a version of the spatially staggered mesh may be attributed to Harlow and Fromm (HF64) although its apparent reinvention over the intervening two decades is an attestation of its efficacy.

In the field of Stirling machine analysis, Urieli (Ur77) applied the temporally coincident, spatially staggered grid to a simple generic machine geometry. Schock, by contrast, used a temporally and spatially coincident grid and invoked volumetrically weighted averages for computing flow rates between grid points (Sc78). Both of these discretisation schemes involve the application of the differential conservation balances in a one-dimensional Eulerian frame of reference.

Although the spatial discretisation scheme used in the simulation model contains elements employed by Urieli and Schock, it has its origins in the mesh structure used in the 'Marker and Cell' (MAC) method of Harlow and Welch (HW65). In particular, the general precepts of a temporally coincident, spatially staggered numerical discretisation scheme suitable for the application of the differential conservation balances are applied to the timeaveraged integral balances described in section 2.3. This application admits 
a three-dimensional space in a combined Eulerian/Lagrangian frame of reference.

As the mass, momentum, and energy balances are fundamentally based upon the concept of a discrete volume, the spatial discretisation scheme naturally devolves to partitioning a given space into an assemblage of discrete volumes with coincident boundaries. All the intensive parameters are thus expressed in volume-averaged terms so that, by definition, the value of any intensive parameter at a point within a discrete volume is extrinsic to the conservation equations. Estimates of the intra-discrete volume intensive parameter distribution may be made using 'volume functions' whose complexity is a function of the boundary conditions imposed on the discrete volume.

The essence of the spatial discretisation scheme involves a method of constructing the discrete volume grid so that, within any discrete volume, scalar intensive parameters are assigned a position and vector intensive parameters are assigned a plane, respectively. This introduces the concept of a 'volumetric filtration' process which may be defined for a scalar field as the assignation of a point to a discrete volume so that volume-averaged scalar intensive parameter at that point satisfies the mean value theorem (TM72) for the discrete volume. Similarly, for a vector field, volumetric filtration assigns a plane to a discrete volume such that the value of a vector intensive parameter over the plane (and perpendicular to it) normalised with respect to the area of the plane satisfies the mean value theorem for the discrete volume.

The characteristics of the discretisation scheme may be illustrated by a two-dimensional Eulerian space using a triangular mesh as shown in figure 2.1. The mass and energy integral balances are applied to a common discrete volume while the momentum balance is applied to discrete volumes which are offset from the mass/energy discrete volume and straddle its boundaries. In this arrangement, the momentum conservation balance is applied to the net momentum, that is, to as many components as the dimensionality of the field requires (two in this case). Typically, it is convenient to resolve the momentum into components perpendicular and parallel to the plane generated by the volumetric filter.

However, although generalised, this scheme is not necessarily the most efficient numerically. Consider, for example, the Cartesian mesh shown in figure 2.2 (the same observations apply to any regular mesh such as cylindrical or spherical). In this case, the net momentum balance may be split into its vector components so that each component balance is applied to a unique offset discrete volume. As indicated by the double cross-hatched area in figure 2.2, the net momentum can be calculated by the vector addition of the momentum components determined individually for that volume. Thus this particular case still yields the net momentum over the entire mass/energy discrete volume as does the general scheme but requires half the computational effort in two dimensions. 


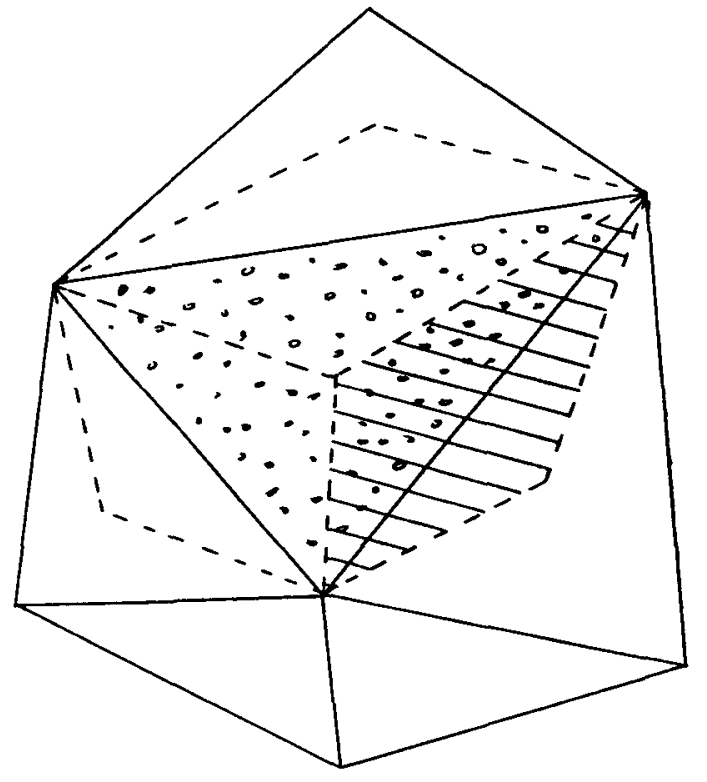

$\because \because \because \because=$ MASSIENERGY DISCRETE VOLUME

Figure 2.1 Spatial discretisation scheme characteristics.

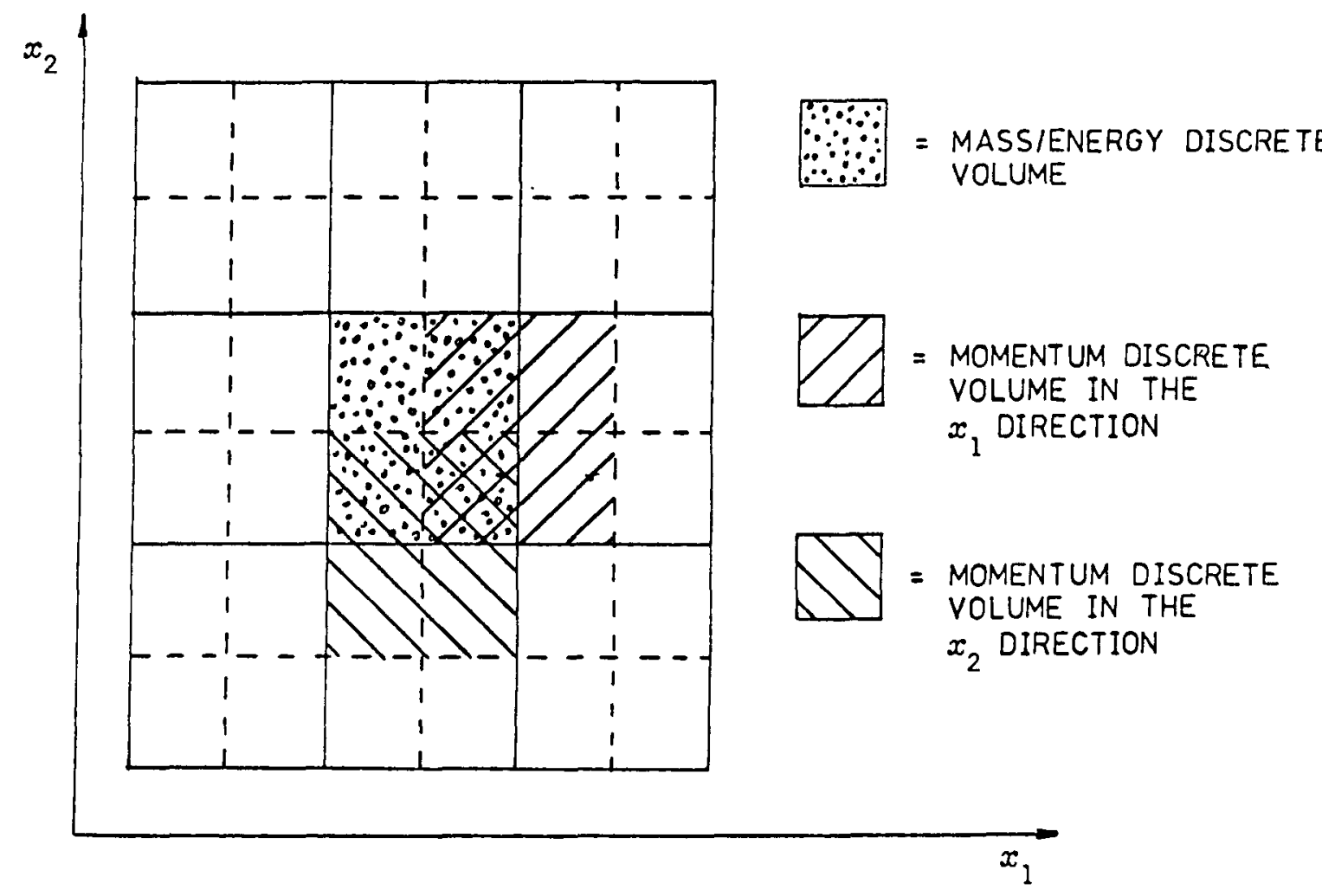

Figure 2.2 Cartesian mesh spatial discretisation. 
Generalising these concepts to a three-dimensional space with an arbitrary coordinate system, the characteristics of the staggered discrete volume grid may be expressed symbolically by denoting the mass/energy discrete volume as $V_{(s)}$ and any momentum discrete volume as $V_{n(s)}$. Then considering any adjacent mass/energy discrete volumes $V_{(s) i}$ and $V_{(s) i+1}$ separated by a boundary $A_{n(s) i+1}$ (where $i$ represents an arbitrary sequence), the following attributes may be defined for the discrete volume grid.

\section{Attribute I}

A momentum discrete volume straddles every mass/energy discrete volume boundary $A_{n(s) i+1}$ and is bounded by a convex surface containing the perimeter of the boundary $A_{n(s) i+1}$ and the volume centroids of the adjacent mass/energy discrete volumes. Symbolically this becomes:

$$
\left[\lambda\left(V_{n(s) i+1}\right) \cap\left\{\lambda\left(V_{(s) i}\right) \cup \lambda\left(V_{(s) i+1}\right)\right\}\right] \subset\left\{\lambda\left(V_{(s) i}\right) \cup \lambda\left(V_{(s) i+1}\right)\right\}
$$

\section{Attribute II}

The volume-averaged intensive parameters corresponding to a mass/energy discrete volume are located at its volume centroid. Thus $[v] \bar{\psi}$ is located at $[\mathrm{v}] \overline{\mathrm{x}}$ such that:

$$
[\mathrm{v}]^{\overline{\mathbf{x}}}=\left(\int_{V} \mathbf{x d V}\right) / V
$$

\section{Attribute III}

The volume-averaged intensive parameters corresponding to a momentum discrete volume are located on the boundary $A_{n(s) i+1}$. Therefore $\left[\mathrm{v}_{\mathrm{n}}\right]^{\bar{\psi}}$ is determined by:

$\int_{V_{\mathrm{n}(s)}} \psi \mathrm{d} V=\int_{\alpha V_{(s) i}} \psi \mathrm{d} V+\int_{\beta V_{(s) i+1}} \psi d \mathrm{~d} V$

where $\alpha, \beta<1$ satisfy equation (2.36). 


\section{Attribute IV}

The net momentum of any mass/energy discrete volume is uniquely defined everywhere in the volume by the momenta of its boundary momentum discrete volumes, or:

$$
\int_{V_{(s)}} g \mathrm{~d} V=\Sigma \int_{V_{n(s)}^{*}} \mathbf{g}_{n} \mathrm{~d} V_{n(s)}
$$

where:

$$
\Sigma \alpha\left(V_{n(s)}^{*}\right)=2\left(V_{(s)}\right)
$$

Applying these attributes to the particular Cartesian mesh of figure 2.2 simplifies equation (2.36) to:

$\alpha^{-1}\left(\lambda\left(V_{(s) i}\right) \cap \lambda\left(V_{n(s) i+1}\right)\right)=V_{(s) i} / 2$

$V_{(s) i}+V_{(s) i+1}=V_{n(s)}$

which satisfies equations (2.37) and (2.39).

Equation (2.37) implies that in the case of the one-dimensional Cartesian coordinate system which is most often used for Stirling machine analysis, a mass/energy discrete volume centroid is defined by a plane separating two adjacent discrete momentum volumes. In a two-dimensional, Cartesian system the centroid becomes a line perpendicular to the mesh surface while only in three dimensions does the centroid become a point.

The attributes of the spatial discretisation scheme enable all the nonvolume-averaged boundary terms in the time-averaged integral balances of equations (2.30) through (2.32) to be replaced with volume-averaged terms, so permitting the balances to be numerically discretised directly. The final forms of the integral balances used in the simulation model are given in combined Eulerian/Lagrangian form by:

Mass :

$\left.\mathrm{d} \bar{M}_{(s)} / \mathrm{d} t=\int \bar{A}_{n(s)}\left\{\left(\operatorname{ttv}_{\mathrm{n}}\right] \overline{\mathrm{g}}-\left[\mathrm{tv}_{\mathrm{n}}\right] \bar{\rho} \overline{\mathrm{v}}_{n(S)}\right) \cdot-\mathrm{n}\right\} \mathrm{d} \bar{A}$ 
where the subscript $n$ denotes that the relevant parameters are associated with the momentum discrete volume.

Momentum:

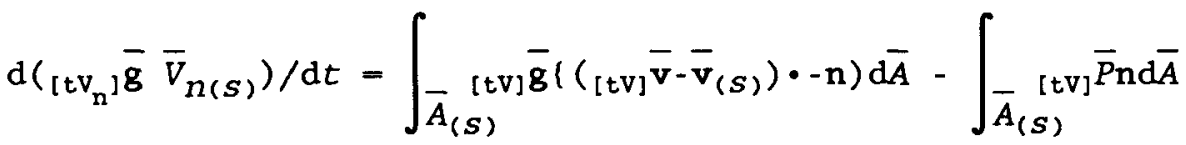

$$
\begin{aligned}
& -\int_{\bar{A}_{(s)}}\left(\left[\mathrm{v} \overline{\mathrm{T}}^{(\mathrm{t})} \cdot-\mathrm{n}\right) \mathrm{d} \bar{A}-\bar{M}_{n(s)} \mathrm{g}\right. \\
& -\int \bar{A}_{(s)}\left(\left(\left[_{[\mathrm{tv}]} \bar{\mu}\left\{\nabla_{[\mathrm{tv}]} \overline{\mathrm{v}}+\left(\nabla_{[\mathrm{tv}]} \overline{\mathrm{v}}\right)^{\mathrm{T}}\right\}\right.\right.\right. \\
& \left.\left.+\left\{\left({ }_{[t v]} \bar{\Lambda}-2_{[t v]} \bar{\mu} / 3\right)\left(\nabla \cdot{ }_{[t v]} \bar{v}\right)\right\} I\right] \cdot-n\right) d \bar{A}
\end{aligned}
$$

where $T$ in equation (2.31) is replaced by the volume and time average of equation (2.9) and the Reynolds stress tensor is given by the correlation:

$[v]^{T^{(t)}}=-\overline{[v] \bar{g}^{\prime}[v]^{\prime}}$

Energy:

$$
\begin{aligned}
& \hat{C}_{V} \mathrm{~d}([\mathrm{tV}] \bar{T} \bar{M}(s)) / \mathrm{d} t=\bar{V}_{(s)}\left\{_{[\mathrm{tV}]} \overline{\overline{\dot{\hat{E}}}}+{ }_{[\mathrm{tV}]} \overline{(\mathrm{T}: \nabla \mathrm{v})}+{ }_{[\mathrm{V}]} \bar{\Phi}^{(\mathrm{t})}\right. \\
& \left.+\left({ }_{[t v]} \bar{v} \cdot \nabla_{[t v]} \bar{P}\right)\right)-{ }_{[t v]} \bar{P} \mathrm{~d} \bar{V}_{(s)} / \mathrm{d} t \\
& -\int_{\bar{A}_{n(s)}\left[t v_{n}\right]} \bar{\kappa}\left(\nabla_{\left[t v_{n}\right]} \bar{T} \cdot-n\right) \mathrm{d} \bar{A}-\int_{\bar{A}_{n(s)}}\left({ }_{\left[\mathrm{lv}_{\mathrm{n}}\right]} \overline{\mathrm{q}}^{(\mathrm{t}) \cdot-\mathrm{n}) \mathrm{d} \bar{A}}\right. \\
& \left.+\hat{C}_{P} \int_{\bar{A}_{n(s)}\left[\mathrm{tv}_{\mathrm{n}}\right]} \bar{T}\left\{\left(\mathrm{Ltv}_{\mathrm{n}}\right] \overline{\mathrm{g}}-\left[\mathrm{tv}_{\mathrm{n}}\right] \mathrm{\rho} \overline{\mathrm{v}}_{n(s)}\right) \cdot-\mathrm{n}\right\} \mathrm{d} \bar{A}
\end{aligned}
$$


where ${ }_{[v]} \bar{\Phi}^{(t)}$ defines the turbulent dissipation tensor which is modelled and the turbulent flux vector takes the form:

$\left[v_{n}\right] \bar{q}^{(t)}=\hat{C}_{P}\left(\overline{\left[v_{n}\right]^{\bar{\rho}}\left[v_{n}\right]^{\prime}{ }_{\left[v_{n}\right]} \bar{T}}-\overline{\bar{\rho}\left[v_{n} \bar{v}^{\prime}\right.}\left[\mathrm{tv}_{n}\right]^{\bar{T}}\right)$

\subsection{THE NUMERICAL SOLUTION ALGOR THM}

An implicit (or advanced time) numerical algorithm is used to temporally integrate the total time-differential conservation balances of equations (2.41) through (2.43). The algorithm has its origins in the Implicit Continuous-fluid Eulerian (ICE) technique of Harlow and Amsden (HA71) and the Semi-Implicit Method for Pressure-Linked Equations, Revised (SIMPLER) of Patankar $(\mathrm{Pa} 80)$.

Central to the algorithm is the notion (HA71) that a change in the pressure field is a function of the information propagation rate, or:

$\Delta P=(\partial P / \partial \rho) T^{\Delta \rho}$

where $(\partial P / \partial \rho)_{T}$ is the square of the isothermal speed of sound for a fluid with constant specific heats. Substituting the equation of state (equation (2.33)) and discretising yields:

$\left({ }_{[\mathrm{tV}]} \bar{P} \bar{V} / R_{[\mathrm{tV}]} \bar{T}-\bar{M}^{\mathrm{s}}\right) / \Delta t \approx \mathrm{d} \bar{M}_{(s)} / \mathrm{d} t$

after multiplying through by $\bar{V} / \Delta t$. Equating with equation (2.41) produces:

$\left.\left.{ }_{[\mathrm{tV}]} P \bar{V} / R_{[\mathrm{tV}]} \bar{T} \Delta t=\bar{M}^{s} / \Delta t+\int_{\bar{A}_{\mathrm{n}(s)}}\left\{\left({ }_{[\mathrm{tv}}\right]_{\mathrm{n}} \overline{\mathrm{g}}-{ }_{[\mathrm{tv}}\right]_{\mathrm{n}} \bar{\rho} \overline{\mathrm{v}}_{n(s)}\right) \cdot-\mathrm{n}\right\} \mathrm{d} \bar{A}$

Equation (2.42.1) may be discretised and rearranged to produce:

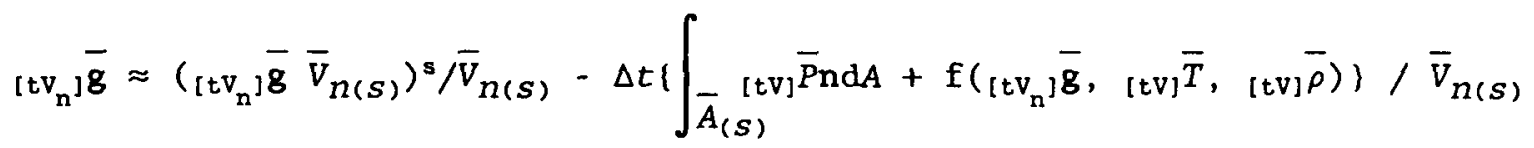


Substituting into equation $(2.46)$ for $\left[\mathrm{tv}_{n}\right]^{\overline{8}}$ and rearranging:

$$
\begin{aligned}
& { }_{[\mathrm{tv}]} \bar{P} \bar{V} / R_{[\mathrm{tv}]} \bar{T} \Delta t+\left(\Delta t / \bar{V}_{n(s)}\right) \int_{\bar{A}_{\mathrm{n}(s)}}\left(\int_{\bar{A}_{(s)}[\mathrm{tv]}} \bar{P} \mathrm{nd} \bar{A} \cdot-\mathrm{n}\right) \mathrm{d} \bar{A}
\end{aligned}
$$

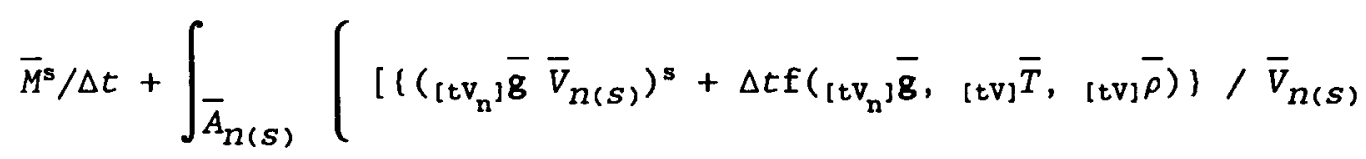

$$
\begin{aligned}
& \left.\left.-\left[\mathrm{tv}_{\mathrm{n}}\right] \bar{\rho} \overline{\mathrm{v}}_{n(s)}\right] \cdot-\mathrm{n}\right) \mathrm{d} \bar{A}
\end{aligned}
$$

Equation (2.47) yields an advanced time or implicit pressure field which is obtained by linking the pressure terms in the conservation of mass and momentum balances, hence the 'pressure-1inked' terminology. However, equation (2.47) can only be solved given the advanced time temperature and density fields which in turn depend primarily on the advanced time mass flux field. This dilemma is resolved by using an iterative solution scheme based on an estimated or guessed mass flux field ${ }_{\left[\mathrm{tv}_{\mathrm{n}}\right]} \overline{\mathrm{g}}^{\star}$ as used in the SIMPLER algorithm.

The set of steps comprising the algorithm may be described broadly as follows (see Go87 for specifics):

1. Guess the mass flux field $\left[\operatorname{tv}_{n}\right]^{\bar{g}^{\star}}$.

2. Explicitly compute the discrete volume masses from equation (2.41) and then infer ${ }_{[\mathrm{t} V]} \bar{\rho}$ from known values of $\bar{V}_{(S)}$.

3. Implicitly compute the temperature field using equations (2.43).

4. Implicitly compute the pressure field from equation (2.47).

5. Implicitly compute the mass flux field from equations (2.42) using the pressure field determined in step 4.

6. Compare $\left[\operatorname{tv}_{n}\right]^{\bar{g}}$ (computed) with $\left[\operatorname{tv}_{n}\right]^{\bar{g}^{*}}$ (guessed) and return to step 2 with $f\left(\left[t v_{n}\right] \bar{g},\left[t v_{n}\right]^{*}\right) \rightarrow\left[v_{n}\right] \bar{g}^{\star}$ if the mass flux field is insufficiently converged.

As with all implicit schemes, this algorithm requires the repeated numerical inversion of matrices. Hence the cost-effectiveness of the algorithm for transient compressible fluid flow simulation is limited by the 
size of the matrices generated. This arises because a break-even point is eventually reached when the cost of matrix inversion becomes equal to the cost of using an explicit algorithm with time steps small enough to satisfy the Courant criterion (CF67). However, for all the problems described in this report, the algorithm is constrained to operate within the cost-effectiveness break-even point by limiting the spatial discretisation (particularly in the two-dimensional problems).

It should be noted that equations (2.45) and (2.47) are discretised using a simple first order temporal difference. In general, this is not necessary and other higher order temporal discretisation schemes may be used (see section 4.7).

\subsection{INFORMATION PROPAGATION MODELS}

The viability of an implicit analysis for compressible fluid flows depends on the extent to which the analysis properly accounts for information propagation both on a cyclic equilibrium as well as on a transient basis. In an explicit analysis, accurate information propagation modelling requires that the Courant criterion (CF67) is met at each discrete volume. This imposes limitations on the integration time increment for a given spatial discretisation. The issue then is to find a similar criterion for selecting the time increment in an implicit analysis. Consider equation (2.47) in the following reduced form:

$$
\sum_{j=i-m}^{i+m} K_{j[t v]} \bar{P}_{j}=\alpha_{i}
$$

where $i$ denotes the individual mass/energy discrete volumes of which the flow area is comprised, $m$ is dependent on the dimensionality of the problem, and $K_{j}$ and $\alpha_{i}$ depend on $\Delta t$. On a transient basis for arbitrary $\Delta t$, equation (2.48) can be applied to a series of pressure domains, one for each discrete mass/energy volume as illustrated (for a particular two-dimensional Eulerian field) in figure 2.3.

Each pressure domain has an extent $\sum_{i} f\left(v \pm v_{a} I\right)_{i} \Delta t$ determined by the information propagation characteristics where $\left(v_{a}\right)_{i}$ is the sonic velocity within each discrete mass/energy volume comprising a particular pressure domain. Hence by this process of partitioning, a 'pressure domain splitting' (PDS) algorithm may be structured to model information propagation phenomena. This simplified explanation ignores the complexities arising from defining the pressure domain boundaries under supersonic or sonic flow conditions (that is, when $\left.|v|_{i} \geq\left(v_{a}\right)_{i}\right)$. However, it may be mentioned that under these conditions the PDS algorithm essentially devolves to a standard approach such as the 'region-to-region' method (Jo69). Unfortunately, the PDS approach is computationally quite expensive and hence may not be practical for Stirling machine analysis. 


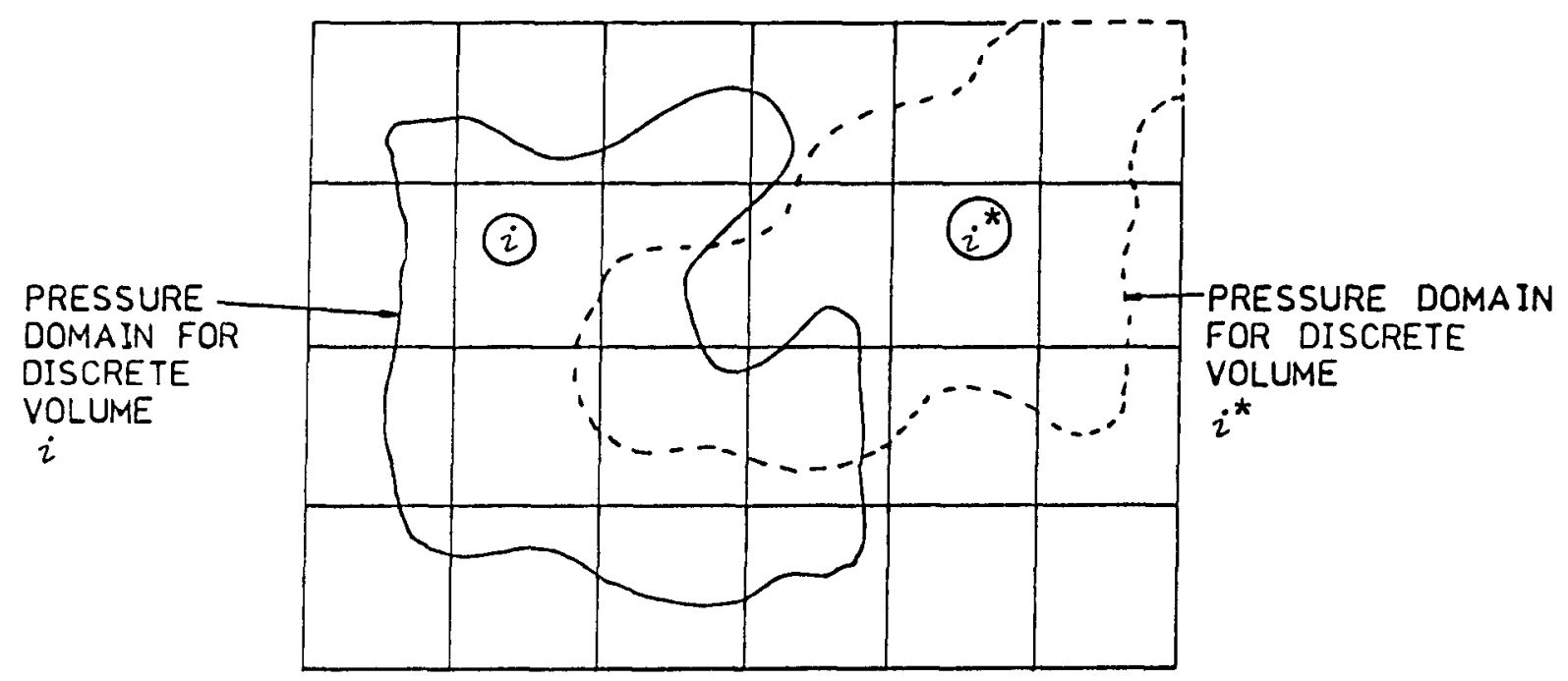

Figure 2.3 Structure of the pressure domain splitting algorithm.

However, the principal focus of Stirling machine simulation is the portrayal of cyclic equilibrium conditions rather than the details of a specific transient cycle. This suggests a significant simplification by applying equation (2.43) to the entire flow field (or treating the flow field as a unitary pressure domain). Two approaches to obtaining the cyclic equilibrium solution under these conditions may be hypothesized:

1. Infinite information propagation.

This hypothesis states that, at cyclic equilibrium, sufficient time has passed such that every point in the flow field has received information from every other point in the flow field for all instants over the cyclic period. The hypothesis may be implemented by arbitrarily selecting an integration time increment that is much less than the smallest information propagation time characteristic of a particular machine. The time characteristic may be defined as the interval required for a pressure wave to exactly traverse the unitary pressure domain once. Henceforward, the infinite information propagation hypothesis is distinguished by referring to its implementation as the 'equilibrium algorithm'.

2. Characteristically determined integration time increment.

Here the integration time increment is treated as a dependent variable which is instantaneously equal to the machine time characteristic. This yields the spatially limiting case of the PDS algorithm and as such approximates the full information propagation transient solution. The implementation of the characteristically determined integration time increment hypothesis is termed the 'unitary pressure domain' (UPD) algorithm. 
The characteristic number $N_{c h}$ is a convenient way of describing the information propagation characteristics of a Stirling machine. $N_{c h}$ is defined as the number of complete pressure traverses between the expansion and compression space piston faces occurring over a cycle or:

$N_{c h}=1 / f \sum_{i}\left[I_{i} / \min \left\{\left|v_{i}+\left(v_{a}\right)_{i}\right|,\left|v_{i}-\left(v_{a}\right)_{i}\right|\right\}\right]$

Hence the larger $N_{c h}$, the more accurate the equilibrium algorithm is likely to be in predicting cyclic equilibrium performance.

\subsection{BOUNDARY ADVECTION}

In the light of the attributes of the spatial discretisation scheme and, in particular, the area normalisation requirement for the volume-averaged momentum, the interpretation of the momentum advection term in equation (2.42.1) is not self-evident. The usual approaches to boundary advection do not translate directly into the discrete volume integral framework where boundary discontinuities are admissible although not necessary. Several approaches to dealing with boundary advection are possible. These include a simple one-dimensional equilibrium approach, a multi-dimensional volume function analysis and flux corrected transport methods such as those of Book, Boris and Hain (BB75) and MacCormack (Mc82). In the simulation model described in this report, the simple one-dimensional equilibrium approach has been adopted as a baseline and modified by empirical models where necessary.

Physical insight to the form of the boundary advection of momentum may be obtained by solving equation (2.42.1) under equilibrium conditions ignoring mutual and external forces and turbulent momentum fluxes, that is:

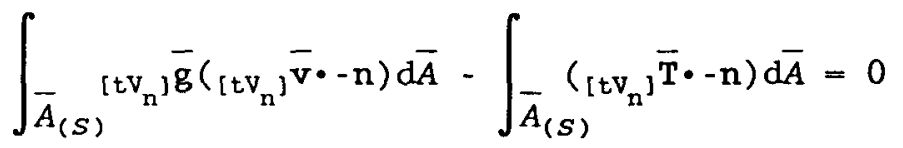

Considering the pair of adjacent discrete momentum volumes shown in figure 2.4 in one dimension, equation (2.50) has the general solution (dropping the averaging notation for clarity):

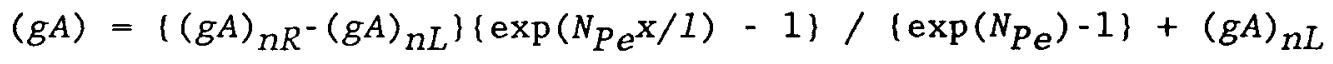




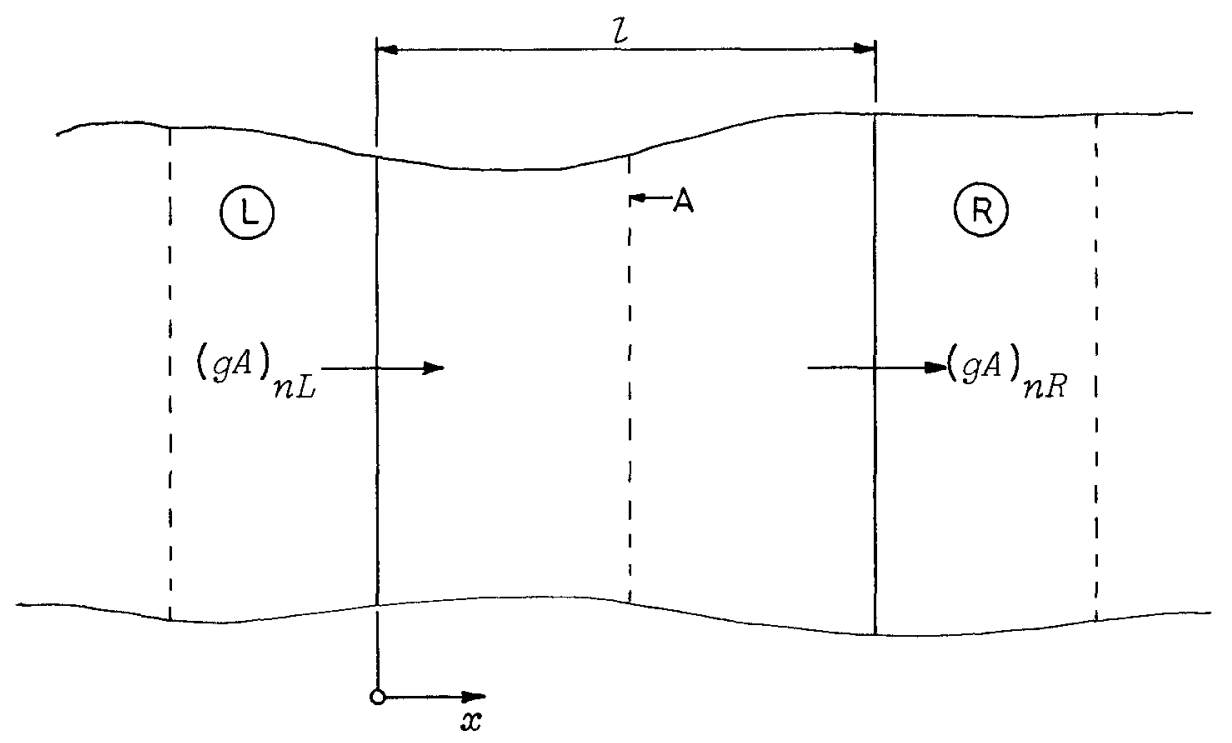

Figure 2.4 Adjacent one-dimensional momentum discrete volumes.

where $N_{P e}$ is the Peclet number at the centroid of the discrete mass/energy volume of length 1 given by:

$N_{P e}=3 \rho v 1 / 4 \mu$

Following Patankar ( $\mathrm{Pa} 80$ ) and denoting the net boundary momentum flow (advection plus diffusion) across boundary $A$ in figure 2.4 as $G$, equations (2.51) produce:

if $N_{P e}=0$ then:

$$
G=(4 \mu / 3 \rho 1)\left((g A)_{n L^{-}}(g A)_{n R}\right)
$$

if $N_{P e} \neq 0$ then:

$$
G=v\left[(g A)_{n L}+\left\{(g A)_{n L}-(g A)_{n R}\right\}\left\{\exp \left(N_{P e}\right)-1\right\}\right]
$$

These equations provide a physically meaningful methodology for determining the boundary advected momentum flux. Consider a plot of $G$ as a function of $N_{P e}$ as shown in figure 2.5. Since $N_{P e}$ expresses the ratio between the advection and diffusion of momentum across a discrete momentum volume boundary, figure 2.5 shows that even at very low $N_{P e}$ the advection term dominates. When $N_{P e}=0$ there is no advection while in the intermediate range 


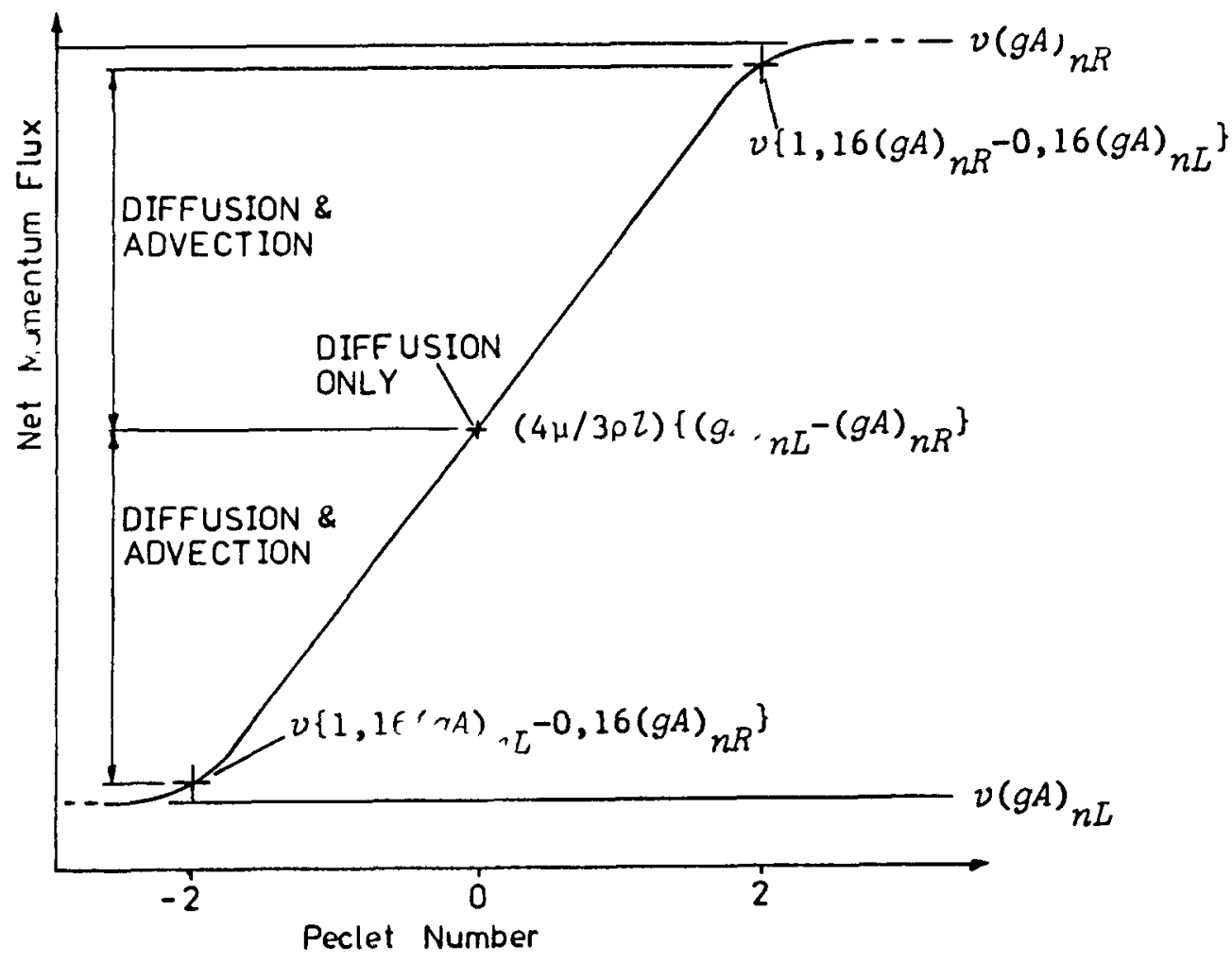

Figure 2.5 Net momentum flux dependence on Peclet number.

the momentum flow is partly diffusive and partly advective. Furthermore, in the 1imit:

As $N_{P e} \rightarrow \infty, G \rightarrow v(g A)_{n L}$

As $N_{P e} \rightarrow-\infty, G \rightarrow v(g A)_{n R}$

Thus figure 2.5 provides a model for determining the boundary advection of momentum. In keeping with the transient nature of Stirling machine fluid flow, the diffusion is kept as a discrete term in equation (2.42.1) and is not lumped together with the advection term. Hence for a discrete momentum volume boundary contained within a discrete mass/energy volume $i$, the advected mass flux is defined in terms of the relative velocity $v_{i}^{*}$ perpendicular to the boundary where:

$v^{*}-[t v]^{\bar{v}}-\bar{v}_{(s)}$

Hence : 
If $v_{i}^{*} \geq 0$ then:

$$
\left.\left.\int \bar{A}_{(s)}[\mathrm{tv}] \bar{g}\left(\mathrm{v}^{*} \cdot-\mathrm{n}\right) \mathrm{d} \bar{A}\right|_{i}=\left(\mathrm{v}^{*}{ }_{[\mathrm{tv}}\right] \overline{\mathrm{g}} \bar{A}_{n(s)}\right)_{i}
$$

If $v_{i}^{*}<0$ then:

$$
\left.\left.\int \bar{A}_{(s)}[\mathrm{tv}] \overline{\mathrm{g}}\left(\mathrm{v}^{*} \cdot-\mathrm{n}\right) \mathrm{d} \bar{A}\right|_{i}=v_{i}^{*}\left({ }_{[\mathrm{tv}}\right] \overline{\mathrm{g}} \bar{A}_{n(s)}\right)_{i+1}
$$

where $i$ and $i+1$ denote adjacent discrete momentum volumes.

Equations (2.54) intrinsically perform the area normalisation required for equation (2.42.1) as mandated by the attributes of the discretisation scheme. This occurs because the transport term is $g A$ (or the mass flow rate) which allows $\left[\operatorname{tv}_{n}\right] \overline{8}$ to be normalised by the appropriate flow area.

It may be noted that equations (2.54) represent a convoluted integral version of the 'second upwind differencing' method proposed by Gentry, Martin, and Daly (GM66). An analysis of this method shows that while it is transportive (as with the classical or first upwind difference), it is also second order accurate for the advection field (Ro82).

The boundary advection of enthalpy in equation (2.43.1) is determined analogously to that described for the momentum equation with a similar end result. Thus for a discrete mass/energy volume boundary contained within a discrete momentum volume $i$, the advected enthalpy flux is defined in terms of the relative boundary mass flux $g_{i}$ perpendicular to the boundary where:

$\mathrm{g}^{*}=\left(\left[\mathrm{tv}_{\mathrm{n}}\right]_{\overline{8}}-{ }_{\left[\mathrm{t} \mathrm{v}_{\mathrm{n}}\right]} \bar{\rho} \overline{\mathrm{v}}_{n(s)}\right)$

Hence:

If $g_{i}^{*} \geq 0$ then:

$$
\left.\int \bar{A}_{\left.n_{1} ;\right)}\left[v_{n}\right] \bar{T}(g \cdot-n) d \bar{A}\right|_{i}=\left(g^{*}[t v] \bar{A}_{n(s)}\right)_{i[t v]} \bar{T}_{i-1}
$$

If $g_{i}^{*}<0$ then: 


$$
\left.\int \bar{A}_{n(s)}^{[\mathrm{tv}}{ }_{\mathrm{n}} \bar{T}(\mathrm{~g} \cdot-\mathrm{n}) \mathrm{d} \bar{A}\right|_{i}=\left(\mathrm{g}^{*}{ }_{[\mathrm{tV}]} \bar{T} \bar{A}_{n(s)}\right)_{i}
$$

where $i-1$ and $i$ denote adjacent discrete mass/energy volumes.

By nature of its simplicity, the approach adopted for modelling transient advection does not resolve completely the 'false diffusion' problem (Ro82) engendered by any upwind scheme. However, in terms of stirling machine simulation, this problem only becomes predominant for the enthalpy advection in the regenerator. Thus in this case, an empirical model is used to overcome the false diffusion deficiency of the advection scheme adopted (section 3.5). More advanced advection modelling schemes based on volume functions have been developed and indeed do yield better results, but in terms of stirling machine simulation, at least in one dimension, it is doubtful whether the improvement in accuracy (which is small when the empirical model is correctly adjusted) is worth the additional cost of computation.

\subsection{CLOSURE}

The foregoing discussion has described the manner in which the fundamental postulates are transformed via a turbulence model and a discretisation scheme into equations suitable for direct implementation in a computer code. The derivation of the final equations places no restrictions on the dimensionality of the implementation--the same equations are applicable to one-, two- and three-dimensional problems. The information propagation model and implicit numerical algorithm developed allow the equations to be integrated temporally so that the influence of information propagation may be bounded. This permits the impact of information propagation effects on the performance of Stirling machines to be at least qualitatively investigated. 
CHAPTER 3

SIMULA T ION O OF THE S PACE POWER

DEMONSTRATOR ENGINE

\section{$3.1 \quad$ INTRODUCTION}

The primary objective in simulating the Space Power Demonstrator Engine (SPDE) was to provide a test bed for validating the turbulence model developed from the Mechanical Engineering Test Rig (METR) experimental results. The SPDE was selected by NASA as the target engine because of its topical relevance to their Stirling engine program and also because it is a more complex device in terms of fluid dynamics than previous generations of freepiston Stirling engines (such as the Sunpower RE1000). This provides a more severe test of the turbulence model and hence increases its potential application envelope.

The simulation approach adopted has been to model the SPDE as a onedimensional system with the option of replacing the one-dimensional heater module with a two-dimensional module. The heater was chosen as the twodimensional replacement module since it was targeted for fluid dynamic similarity with the METR. The purpose of performing a two-dimensional simulation of the heater is to determine on a systems basis the effect of using one-dimensional friction factor and heat transfer coefficient correlations in a Stirling heat exchanger in comparison with a model that does not require such first order empiricism. Nevertheless, the requirement for second order empiricism, in particular the use of turbulence models, remains. The development of such a model is described in chapter 5.

In view of the long lead times expected (and later realised) in producing validation-quality METR experimental data upon which an oscillating flow turbulence model could be based, prudence dictated, that the SPDE simulation codes be developed first using standard friction factor and heat transfer coefficient correlations for the one-dimensional components. Furthermore, the initial two-dimensional heater module simulations would not include any turbulence modelling, that is, the flow is assumed to be laminar. This provides the necessary baseline against which the effect of a turbulence model (as well as any improved one-dimensional correlations based thereon) may be compared.

A comparison of one- and two-dimensional heater module simulations also provides = basis against which the efficacy of two-dimensional simulation of Stirling machines can be judged, that is, whether the improved simulation accuracy is worth the increased cost of computation. Such a judgement is not obvious because it can be hazardous to isolate the effects of oscillating flow in Stirling machine heat exchangers on the overall machine performance. This 
arises since the cyclic energy balance is the product of the synergistic interaction of several factors including:

- porous flow in the regenerator,

- compressibility effects, particularly the influence of information propagation, and

- multi-dimensional flow fields in the working space cylinders and heat exchanger plena.

Hence it is possible that in a systems context, the advantages of single component two-dimensional simulation are nullified by the transport boundary conditions imposed by the rest of the closed system. This is an important consideration in attempting to apply any correlations experimentally derived from the METR directly to Stirling hardware because of the boundary condition dissimilarities.

Thus, the intention of the one- and two-dimensional simulations of the SPDE discussed hereafter is to provide an analytic foundation based on actual hardware performance for later application of the METR experimental data. The validity of this foundation is assessed by a comparison of the baseline oneand two-dimensional simulation predictions against the cyclic performance data produced by the SPDE test program. Unfortunately, for the reasons noted in chapter 1 , it did not prove possible to include the turbulence model developed in chapter 5 into the SPDE two-dimensional simulation, nor was it possible to go one step further and develop one-dimensional correlations from either the simulated or analytic turbulence data.

\subsection{SIMULATION HARDWARE}

A schematic of the SPDE is shown in figure 3.1. The engine consists of a pair of back-to-back, beta-configuration, free-piston Stirling engines which share a common expansion space. Work is extracted via linear alternators attached to the pistons. As the component free-piston engines are symmetrical, only half the SPDE need be simulated when using a one-dimensional system model even with the inclusion of a two-dimensional heater module. The SPDE has a design oscillating frequency of $105 \mathrm{~Hz}$ and a pressurization of 150 bars.

The expansion and compression spaces of the SPDE are quite complex in comparison with those of typical Stirling machines such as the Sunpower RE1000 (Sc83) and GM-GPU3 (Th79) engines. Part of this complexity stems from a restriction of the maximum piston and displacer amplitudes to about $12.7 \mathrm{~mm}$. The resulting bore-to-stroke ratios predicate that the displacer and piston function more as flat-plate oscillators than as conventional pistons. For this reason, the flow in the expansion space / heater plenum (figure 3.1) is strongly two-dimensional with a significant radial gradient of axial velocity at the heater entrance. The compression space also exhibits notable twodimensional momentum boundary conditions in addition to an unusual geometry, 


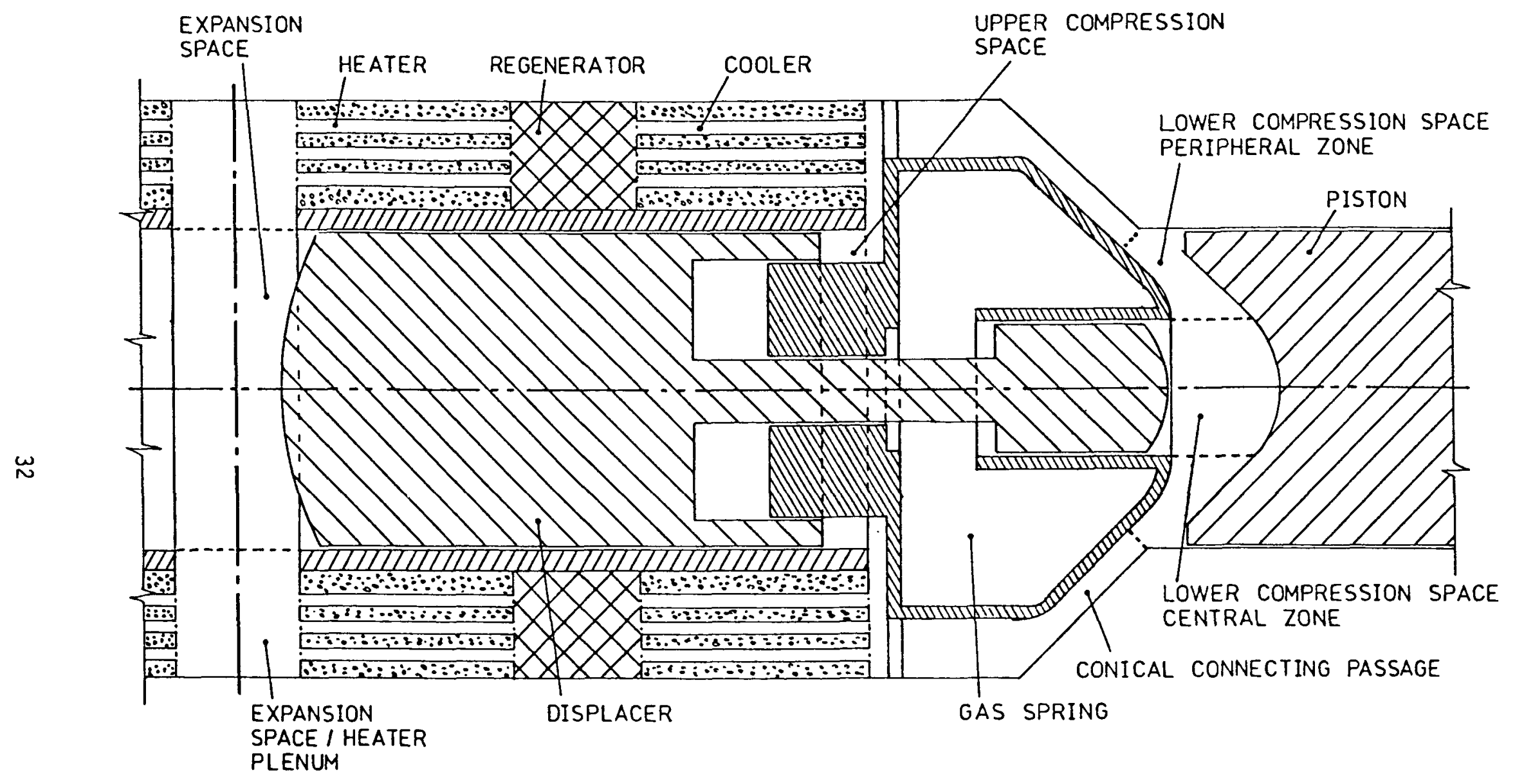

Figure 3.1 Schematic of the Space Power Demonstrator Engine. 
namely, two variable volume components joined by a conical annular passage. Therefore, in the light of these complications and the importance of correctly accounting for two-dimensional momentum boundary conditions (Go87), a strictly one-dimensional system model is not a satisfactory description of the SPDE.

From a fluid dynamics perspective, the balance of the SPDE is of fairly conventional design. A tubular heater and cooler are separated by a square mesh, woven screen regenerator. The displacer is supported via a gas spring while the piston oscillates against a bounce space. Gas bearings are used throughout and a bearing gas supply system is built into the engine. As these details are not essential for implementing the simulation (which relies on predefined displacer and piston harmonic motions), the reader is referred to reference $\mathrm{Br} 87$ for further information.

\subsection{THE SPDE SYSTEM MODEL}

The overall system model of the simulated SPDE is shown in figure 3.2 while a listing of the specific geometry used is given in table 3.1. The regenerator and cooler are treated as one dimensional while the heater may be one or two dimensional. The expansion space is split into a cylinder cavity and a heater plenum. The compression space is divided into upper and lower variable volume components joined by a conical connecting passage which is also given a one-dimensional discretisation.

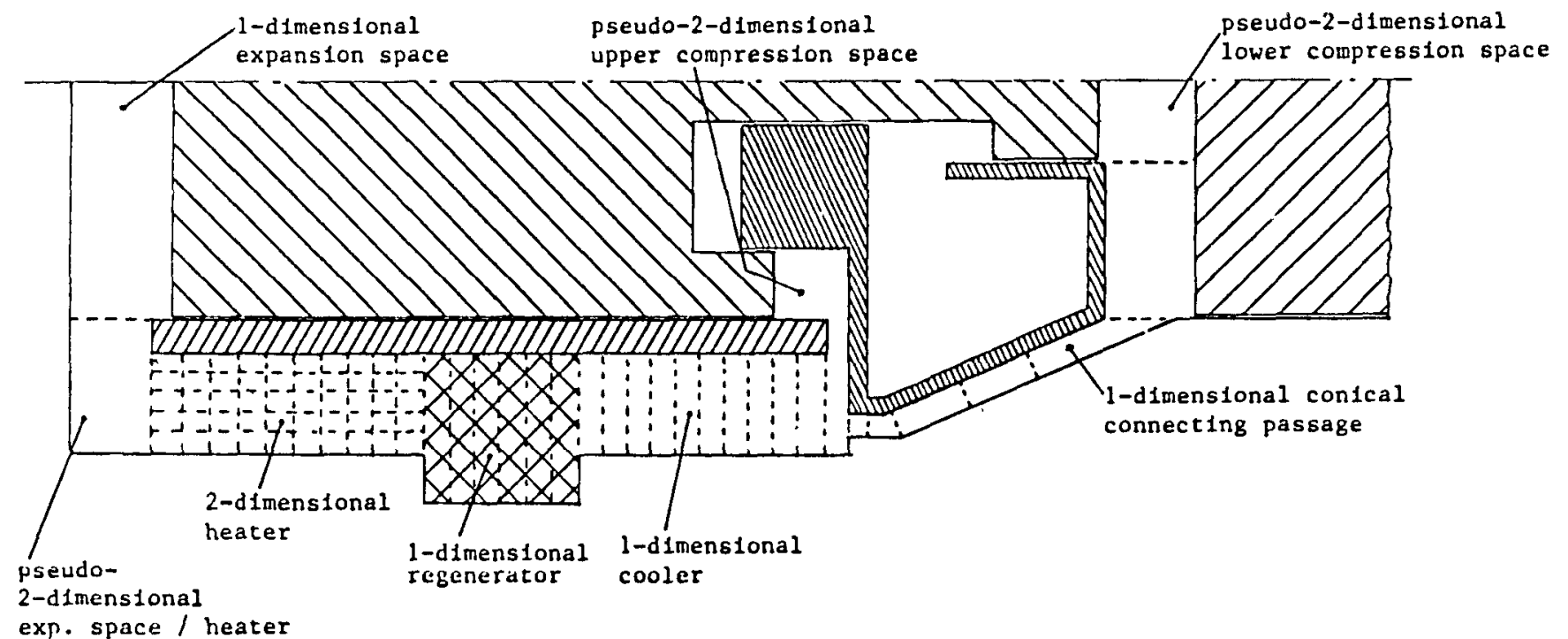

exp. space / heater

plenum

Figure 3.2 SPDE system simulation model. 
Table 3.1 SPDE simulation geometry.

\section{Expansion Space:}

Length

Diameter

Wetted area

Volume at displacer datum

Heater Plenum:

Axial length

Axial flow area

Axial wetted area

Volume

Radial length

Radial flow area

Radial wetted area

Radial entrance area

Volume within radial momentum control volume

\section{Heater:}

No. of tubes

1632

Length

Hydraulic diameter

Regenerator:

Casing inner diameter

Casing outer diameter

Length

No. of layers in gauze stack

Gauze mesh

Gauze wire diameter

Volumetric porosity

Gauze density

Gauze specific heat capacity

\section{Cooler:}

No. of tubes

Length

Hydraulic diameter

Upper Compression Space:

Length

Hydraulic diameter

Midpoint flow area

Upstream boundary flow area

Wetted area

Displacer area

Volume at displacer datum

Axial momentum volume

\section{Flange Passage:}

Length

Hydraulic diameter

Midpoint flow area

Upstream boundary flow area

Wetted area

Volume
$57.24 \mathrm{~mm}$

$114.4016 \mathrm{~mm}$

$12.86 \mathrm{~cm}^{2}$

$57.1 \mathrm{~cm}^{3}$

1c. $16 \mathrm{~mm}$

$289.44 \mathrm{~cm}^{2}$

$71.344 \mathrm{~cm}^{2}$

$298.92 \mathrm{~cm}^{3}$

$54.515 \mathrm{~mm}$

$53.944 \mathrm{~cm}^{2}$

$268.77 \mathrm{~cm}^{2}$

$36.544 \mathrm{~cm}^{2}$

$123.32 \mathrm{~cm}^{3}$
$90.17 \mathrm{~mm}$

$1.27 \mathrm{~mm}$

$139.6746 \mathrm{~mm}$

$223.5708 \mathrm{~mm}$

$25.4 \mathrm{~mm}$

350

200 ins $^{-1}$

$0.04064 \mathrm{~mm}$

0.7105

$7833.03 \mathrm{~kg} / \mathrm{m}^{3}$

$502.42 \mathrm{~J} / \mathrm{kg} . \mathrm{K}$

1584

$95.25 \mathrm{~mm}$

$1.524 \mathrm{~mm}$

$3.81 \mathrm{~mm}$

$21.656 \mathrm{~mm}$

$238.3 \mathrm{~cm}^{2}$

$115.95 \mathrm{~cm}^{2}$

$41.462 \mathrm{~cm}^{2}$

$46.259 \mathrm{~cm}^{2}$

$89.791 \mathrm{~cm}^{3}$

$89.791 \mathrm{~cm}^{3}$

$24.13 \mathrm{~mm}$

$15.457 \mathrm{~mm}$

$33.042 \mathrm{~cm}^{2}$

$33.042 \mathrm{~cm}^{2}$

$293.71 \mathrm{~cm}^{2}$

$84.102 \mathrm{~cm}^{3}$ 
Table 3.1 (continued) SPDE simulation geometry.

\begin{tabular}{ll}
\hline Linking Annulus: & $10.846 \mathrm{~mm}$ \\
Length & $14.566 \mathrm{~mm}$ \\
Hydraulic diameter & $40.66 \mathrm{~cm}^{2}$ \\
Midpoint flow area & $32.086 \mathrm{~cm}^{2}$ \\
Upstream boundary flow area & $121.11 \mathrm{~cm}^{2}$ \\
Wetted area & $44.101 \mathrm{~cm}^{3}$ \\
Volume & \\
\hline Joining Ring Passage: & $61.488 \mathrm{~mm}$ \\
\hline Length & $11.683 \mathrm{~mm}$ \\
Hydraulic diameter & $32.086 \mathrm{~cm}^{2}$ \\
Midpoint flow area & $32.086 \mathrm{~cm}^{2}$ \\
Upstream boundary flow area & $656.73 \mathrm{~cm}^{2}$ \\
Wetted area & $191.81 \mathrm{~cm}^{3}$ \\
Volume & \\
\hline Peripheral Compression Space: & $266.31 \mathrm{~mm}$ \\
Midpoint flow diameter & $35.156 \mathrm{~cm}^{2}$ \\
Midpoint flow area at piston datum & $266.31 \mathrm{~mm}$ \\
Upstream flow diameter & $35.156 \mathrm{~cm}^{2}$ \\
Upstream flow area at piston datum & $455.72 \mathrm{~mm}$ \\
Wetted surface diameter & $355.58 \mathrm{~cm}^{2}$ \\
Wetted surface area at piston datum & $108.83 \mathrm{~cm}^{2}$ \\
Piston area & $169.73 \mathrm{~cm}^{3}$ \\
Volume at piston datum & \\
\hline Central Compression Space: & $42.385 \mathrm{~mm}^{2}$ \\
\hline Length area & $144.98 \mathrm{~cm}^{2}$ \\
Wetted area & $55.851 \mathrm{~cm}^{2}$ \\
Displacer area & $56.438 \mathrm{~cm}^{2}$ \\
Piston area & $242.91 \mathrm{~cm}^{3}$ \\
Volume at piston and displacer datums & \\
\hline
\end{tabular}

Initially, attempts were made at using a strictly one-dimensional description of the upper and lower compression spaces as well as the expansion space. However, as expected (Go87), this approach was not successful in enabling the experimental cyclic performance data to be matched. Hence a "pseudo-two-dimensional" method of including the actual two-dimensional momentum boundary conditions was developed. This method is illustrated in figure 3.3. Figure 3.3.1 shows an expanded view of the expansion space cylinder cavity and heater plenum. The mass fluxes at planes $A$ and $B$ are evaluated using sequential one-dimensional momentum control volumes. However, these orthogonal mass flux vectors are advectively and diffusively decoupled from each other in compliance with the two-dimensional topology. Similarly, the one-dimensional boundary condition within the cylinder for the mass flux computed at plane A is $g_{r}=0$. Similar principles hold in figure 3.3.2 for the upper compression space in which radial and axial velocity and mass flux components are maintained in their proper vectorial relationship. The pseudotwo-dimensional discretisation of the lower compression space shown in figure 3.3.3 is achieved by dividing the space into peripheral and central zones. A one-dimensional mass flux is thus computed within the lower compression space based on physically appropriate boundary conditions. 

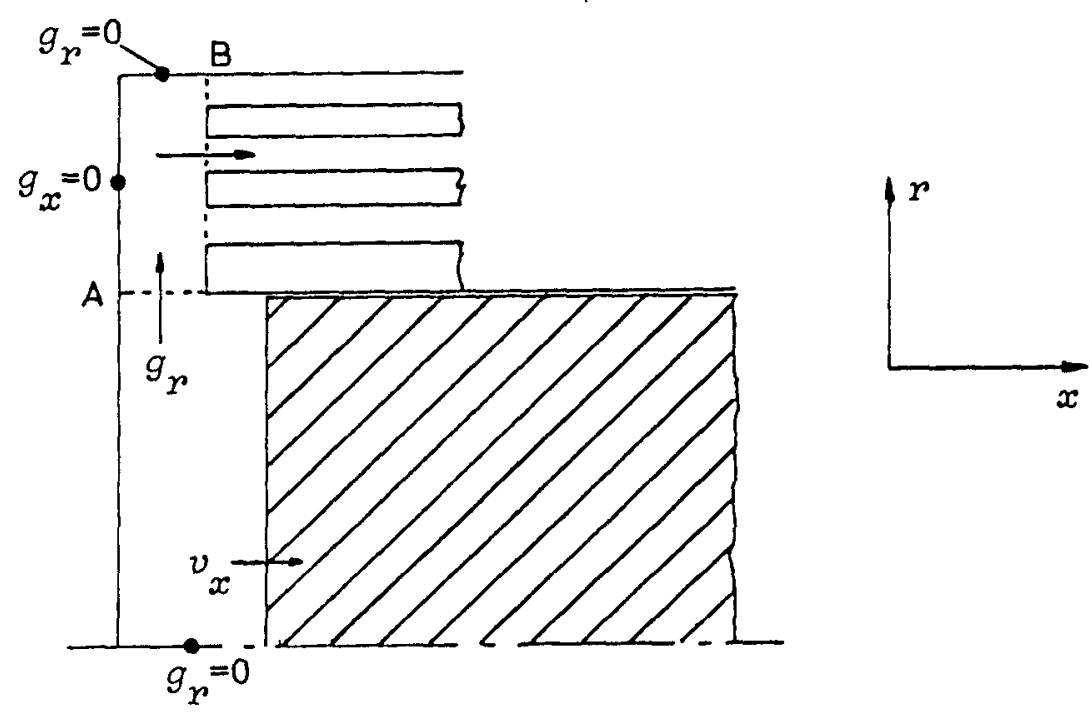

Figure 3.3.1 Expansion space / heater plenum pseudo-two-dimensional discretisation.
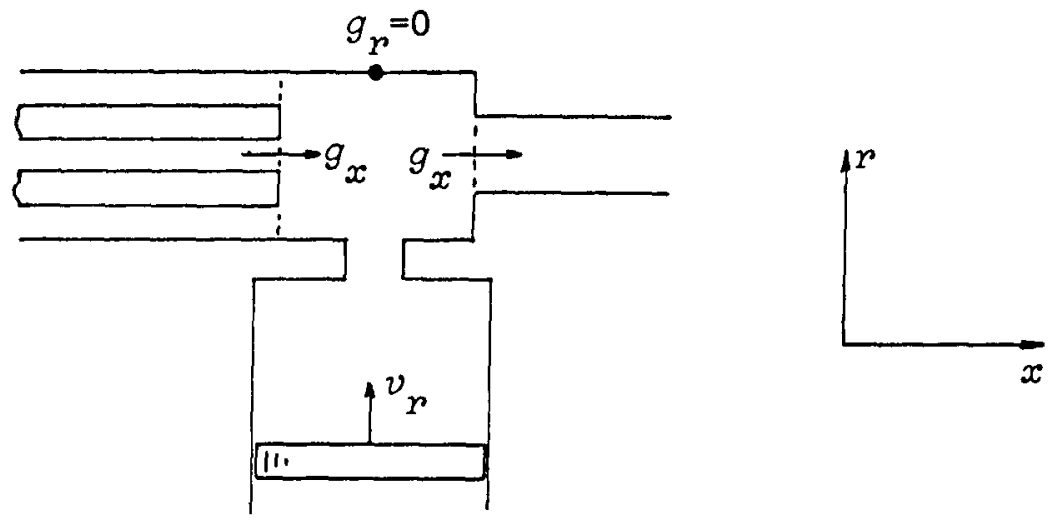

Figure 3.3.2 Upper compression space pseudo-two-dimensional discretisation.

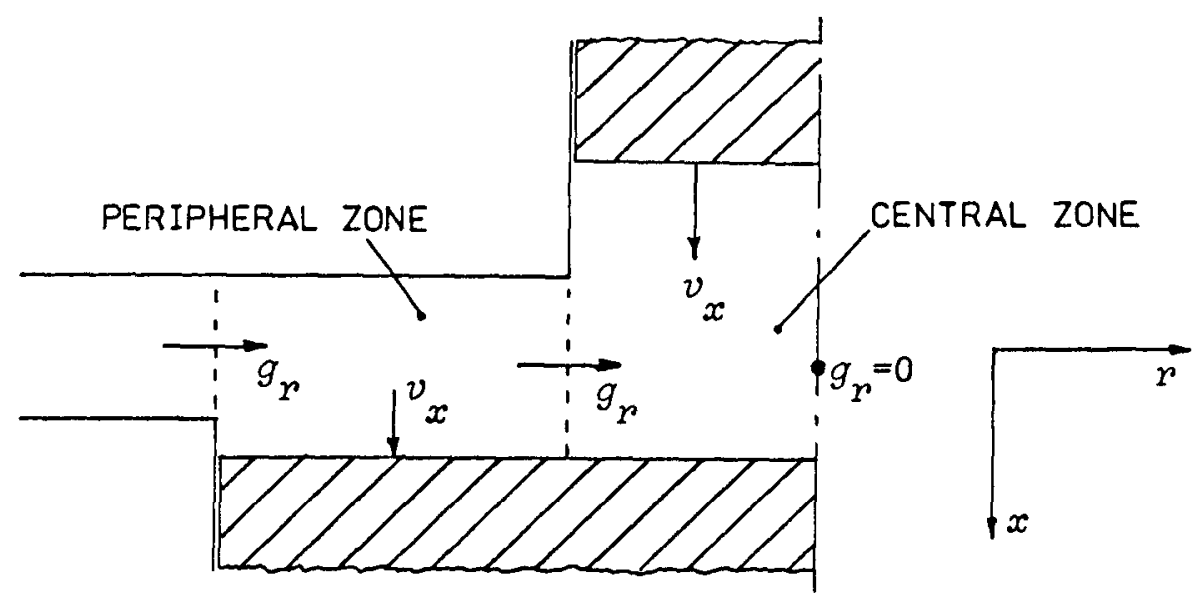

Figure 3.3.3 Lower compression space pseudo-two-dimensional discretisation. 
In the two-dimensional heater module, the heater is described as a twodimensional parallel aggregation of the 1632 separate tubes of which it is composed. Hence the two-dimensional flow parameters calculated for any control volume in the single aggregated tube are assumed to prevail in all the tubes. This assumption is nnt really satisfactory, in view, for example, of the radial gradient of axial locity that exists in the heater plenum for positive gas flows (expansior. to compression space). This implies that the flow in the inner rows of tubes i likely to be rather different from those in the outer rows of tubes, particul. 1y in terms of turbulence triggering effects. However, the better altes lative of specifying several parallel twodimensional heater flow paths was $n t t$ a pragmatic alternative owing to the preliminary nature of this investigation combined with the large computation costs involved. Such an approach deserves to be tested in the future.

The system is discretised spatially by assigning 12, 7, and 3 control volumes to the regenerator, cooler, and conical connecting passage, respectively. The heater is modelled using 7 axial and 6 radial control volumes while all the remaining components are represented by single control volumes.

\subsection{SIMULATION CONSIDERATIONS}

The standard set of equations described in section 2.4 is used. In two dimensions, the equations are mapped onto a cylindrical coordinate system in the heater module. Hence, all the control volume parameters in the twodimensional heater module naturally reflect its inherent radial symmetry as shown in figure 3.4 .

Every mass/energy control volume is associated with two axial ( $x$ ) and two radial $(r)$ momentum control volumes in accordance with the discretisation attributes listed in section 2.4. A linear radial spatial discretisation (constant $\Delta r$ ) was selected because, computationally, it is a more severe test of the simulation than other physically more attractive schemes (such as a logarithmic radial discretisation with the radius decreasing towards the tube wal1). This arises because not biasing the radii to discretise more accurately the steeper boundary layer velocity gradients stresses the volumeaveraging procedure inherent in the integral equations more severely, thus increasing the likelihood of any errors in the simulation becoming manifest. This is particularly true in terms of testing the generality and viability of a turbulence model in an integral framework. 


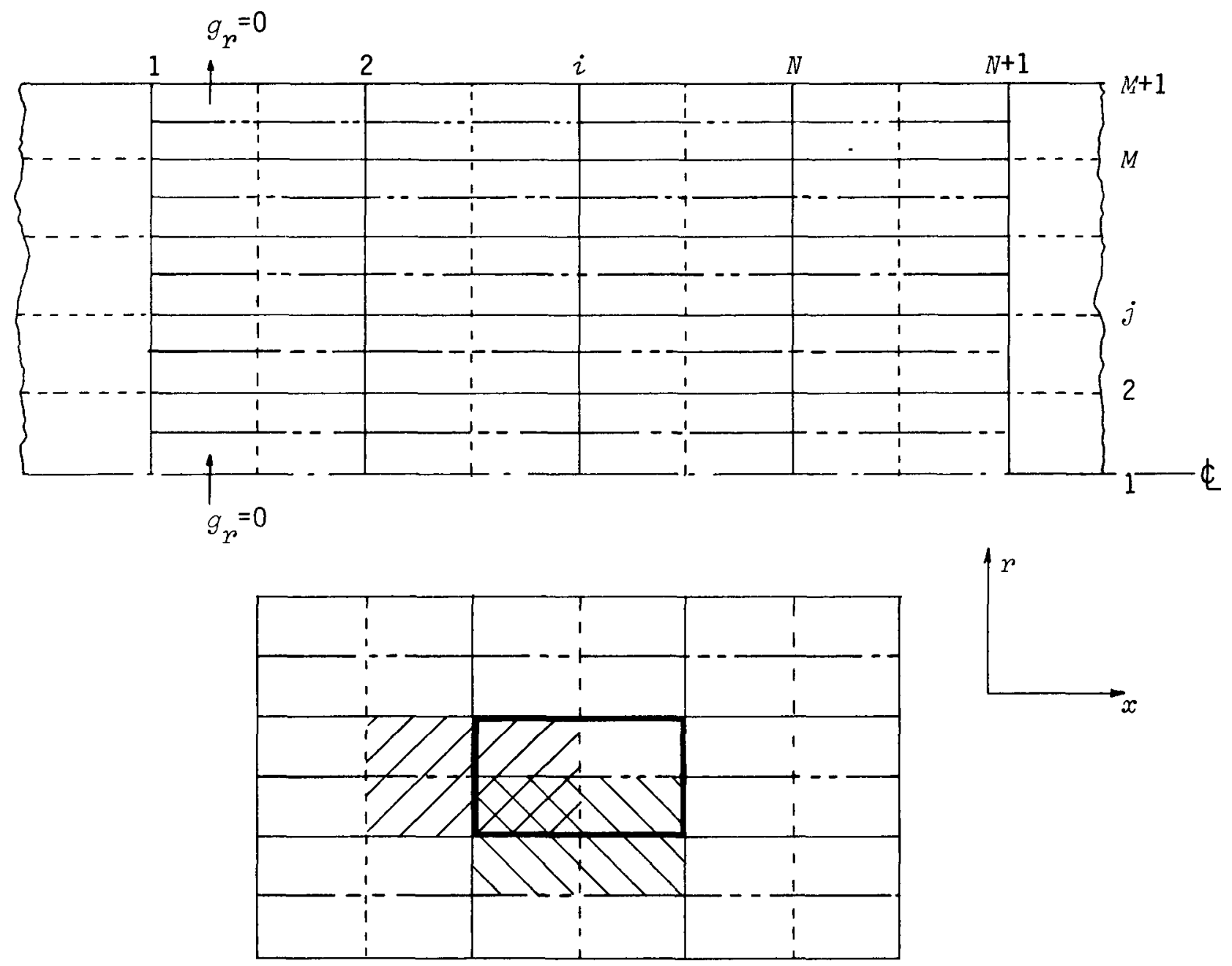

$\square=$ MASS/ENERGY DISCRETE VOLUME $P C A=x$ MOMENTUM DISCRETE VOLUME $\Delta D=r$ MOMEntum oiscrete volume

Figure 3.4 Two-dimensional heater module spatial discretisation. 
The radial momentum flux boundary values are defined as follows:

Tube wal1: $\left(g_{n r}\right)_{i, M+1}=0$

Axis of symmetry: $\left(g_{n r}\right)_{i, 1}=0$

LHS boundary:

$$
\begin{aligned}
\text { if } . & 5\left\{\left(g_{n x} A_{n x}\right)_{1, j}+\left(g_{n x} A_{n x}\right)_{1, j-1}\right\} \geq 0: \\
& \left(g_{n r}\right)_{1, j}=0 \\
\text { if } . & 5\left\{\left(g_{n x} A_{n x}\right)_{1, j}+\left(g_{n x} A_{n x}\right)_{1, j-1}\right)<0: \\
& \left(g_{n r}\right)_{1, j}-f\left\{\left(g_{n r}\right)_{1, j}\right\}
\end{aligned}
$$

RHS boundary:

$$
\begin{aligned}
& \text { if } .5\left\{\left(g_{n x} A_{n x}\right)_{N+1}, j+\left(g_{n x} A_{n x}\right)_{N+1}, j-1\right\}<0: \\
& \quad\left(g_{n r}\right)_{N+1, j}=0 \\
& \text { if } .5\left\{\left(g_{n x} A_{n x}\right)_{N+1}, j+\left(g_{n x} A_{n x}\right)_{N+1}, j-1\right\} \geq 0: \\
& \left(g_{n r}\right)_{N+1, j}=f\left\{\left(g_{n r}\right)_{N+1, j}\right\}
\end{aligned}
$$

The interface between the one-dimensional and two-dimensional meshes is shown in figure 3.5 in terms of an aggregate two-dimensional control volume on the expansion side of the heater (the regenerator side interface is a mirror image of the one shown).

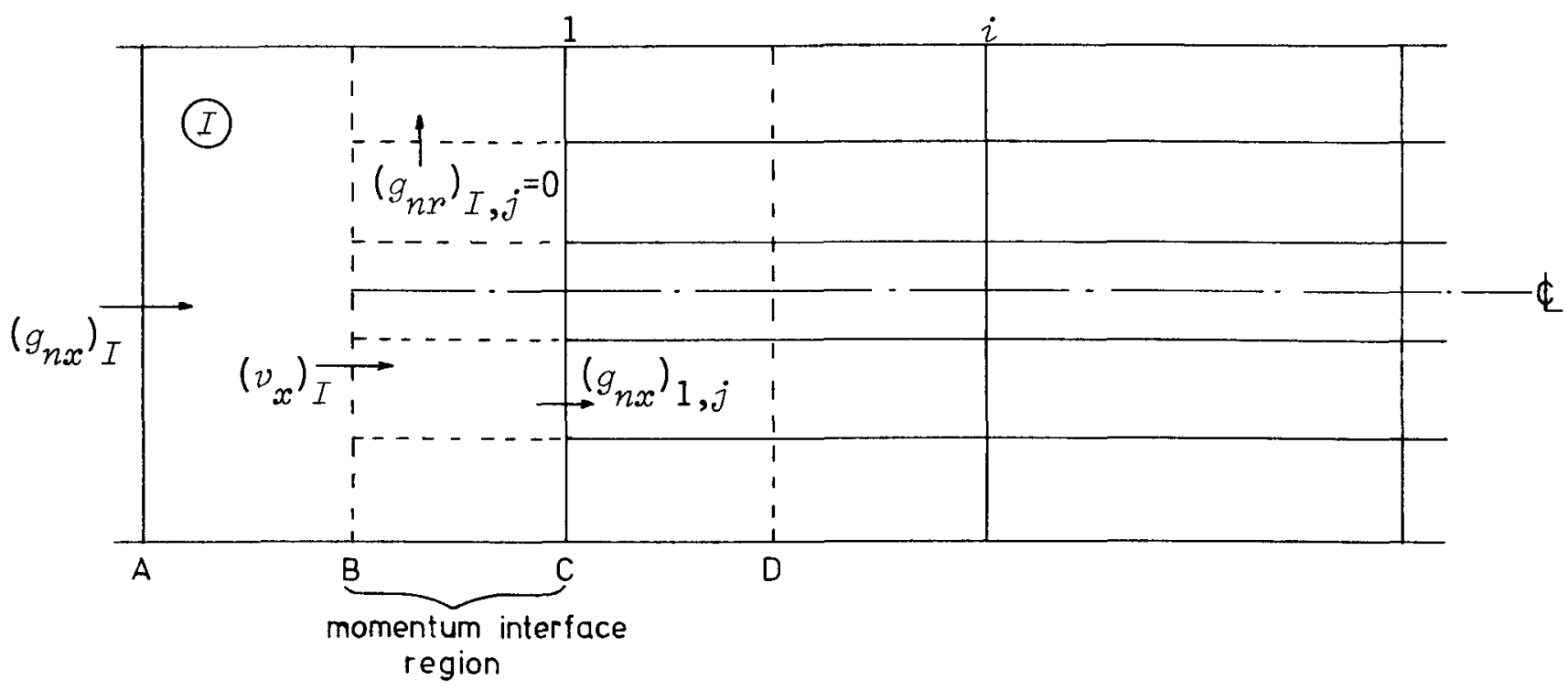

Figure 3.5 Interface between the one- and two-dimensional meshes. 
The mass and energy transport interfaces between the one-dimensional control volume $I$ and the two-dimensional control volumes $I+1, j$ (where $I+1 \Leftrightarrow\{i=1\}$ ) are accomplished naturally in terms of the two-dimensional advective fluxes $\left(g_{n x}\right)_{1, j}$ at plane $C$ and the diffusive fluxes defined by the properties at planes $B$ and $D$ and the distance between them perpendicular to plane $C$. This arises from the discretisation attributes (section 2.4) which locate the volume-averaged intensive properties of control volume $I$ at plane $B$ (as opposed to a point, which would only occur, by definition, in a threedimensional discretisation). The radial momentum interface advective fluxes are defined by equations (3.1) and by noting that $\left(g_{n r}\right)_{I, j} \equiv 0$ in the onedimensional control volume $I$. Diffusive radial momentum interface fluxes are ignored because of their smallness (compared with the advective fluxes) and because of the arbitrariness of the definition $\left(g_{n r}\right)_{I, j} \equiv 0$ (which is, of course, untrue in reality).

The $\mathbf{x}$ momentum interface is more problematic because the definition of the advective velocities $\left(v_{\boldsymbol{x}}\right)_{I, j}$ in the one-dimensional control volume $I$ is somewhat arbitrary. The diffusive flux is computed naturally from the gradient between $\left(g_{n x}\right)_{1, j}$ at plane $C$ and $\left(g_{n x}\right)_{I}$ at plane A. After numerical experimentation, the approach finally adopted for the advective flux interface is defined via $\left(v_{x}\right)_{I, j}$ for the general case $\left\{\left(A_{n x}\right)_{I} \neq\left(A_{x}\right)_{I} \neq\left(A_{n x}\right)_{I+1}\right\}$ by:

$\left.\left(v_{x}\right)_{I, j}=\left(A_{n x}\right)_{1, j}\left\{\left(A_{n X}\right)_{I}\left(g_{n x}\right)_{I} /\left(A_{n x}\right)_{I+1}+\left(g_{n x}\right)_{1, j}\right)\right\} / 2 \rho_{I}\left(A_{x}\right)_{I, j}$

This formulation is mass conservative while allowing $\left(v_{\mathbf{x}}\right)_{I, j}$ to vary radially. The other basic formulation is also mass conservative but assigns a single value to all $\left(v_{x}\right)_{I, j}$. This approach did not fare as well as the approach adopted in tracking the boundary layer growth.

Standard Kays and London (KL64) friction factor and heat transfer correlations are used in all the one-dimensional control volumes (including those in the one-dimensional heater module) while all the turbulent terms in the two-dimensional equations are zeroed, that is:

$\left[V \bar{T}^{(t)}={ }_{\left[V_{n}\right.} \bar{q}^{(t)}={ }_{[V]} \bar{\Phi}^{(t)}=0\right.$

\section{$3.5 \quad$ RESULTS}

The two experimental test points used for the validation exercise are defined by the parameters listed in table 3.2. Test 46 represents a lower power point while test 42 approaches the maximum power output of the SPDE. As the test configuration consists of two back-to-back engines, the wall temperatures as well as the piston and displacer amplitudes are taken as the mean of the left-and right-hand engine parameters. 
Table 3.2 Simulation input parameters.

SPDE Test 46

SPDE Test 42

$(14 / 3 / 86)$

$(11 / 9 / 86)$

\begin{tabular}{|c|c|c|}
\hline Working fluid & Helium & Helium \\
\hline Frequency $(\mathrm{Hz})$ & 99.385 & 99.569 \\
\hline Charge pressure (bar) & 149.67 & 150.29 \\
\hline Heater wall temperature ${ }^{a}(\mathrm{~K})$ & 574.555 & 677.89 \\
\hline Cooler wall temperature ${ }^{a}(\mathrm{~K})$ & 308.052 & 345.11 \\
\hline Displacer amplitude ${ }^{a}(\mathrm{~mm})$ & 6.927 & 7.8016 \\
\hline Piston amplitude $(\mathrm{mm})$ & 6.9255 & 9.1276 \\
\hline
\end{tabular}

Notes:

a. Taken to be the mean of the left- and right-hand engine test parameters.

Three simulations were carried out for each test point, UPD and equilibrium algorithm simulations for the one-dimensional heater module and an equilibrium algorithm simulation for the two-dimensional heater module. The non-dimensional parameters and correction factors associated with the simulation runs are listed in table 3.3. All the friction factor and heat transfer coefficient multipliers operating on the Kays and London correlations are at their baseline values of unity with the exception of those for the regenerator matrix friction factor. These latter multipliers reduce the nominal steady-state friction factor in the regenerator by $35 \%$ and $45 \%$ for tests 46 and 42 , respectively, in order to match the experimental data according to the validation protocol developed in Go87. This is thought to be a consequence of radially non-uniform mass fluxes in the regenerator (owing to entrance effects) and the high frequency of the flow oscillation.

In view of the large Reynolds numbers resultant from the high frequency and pressurisation of the SPDE flow field, the false diffusion problem in the enthalpy advection computation (equations (2.55)) becomes problematic in the regenerator and leads to significant errors in the simulated heater and cooler heat transfers. This indicates the appropriateness of activating a regenerator enthalpy transport model of the kind defined by figure 3.6. In this model, linear upwind spatial extrapolations of the volume-averaged temperature field lead to a better approximation of the actual advected temperatures within the regenerator than those determined from equations (2.55). The particulars of the model are given by equations (3.4) as follows (the averaging notation has been dropped for clarity): 
Table 3.3 Simulation non-dimensional parameters and empirical correlation factors.

\begin{tabular}{|c|c|c|c|c|c|c|}
\hline & \multicolumn{3}{|c|}{ SPDE Test $46(3 / 14 / 86)$} & \multicolumn{3}{|c|}{ SPDE Test $42(9 / 11 / 86)$} \\
\hline Characteristic no. & 24.3 & - & - & 25.7 & - & - \\
\hline Maximum Mach no.a & 0.020 & 0.020 & 0.020 & 0.033 & 0.032 & 0.032 \\
\hline Maximum Reynolds no." & 312944.7 & 310148.1 & 309751.4 & 361334.5 & 350231.2 & 349619.3 \\
\hline $\begin{array}{l}\text { Global tube friction } \\
\text { factor multiplier }\end{array}$ & 1. & 1. & 1. & 1. & 1. & 1. \\
\hline $\begin{array}{l}\text { Regenerator matrix friction } \\
\text { factor multiplier }\end{array}$ & 0.65 & 0.65 & 0.65 & 0.55 & 0.55 & 0.55 \\
\hline $\begin{array}{l}\text { Regenerator matrix heat transfer } \\
\text { coefficient multiplier }\end{array}$ & 1. & 1. & 1. & 1. & 1. & 1. \\
\hline $\begin{array}{l}\text { Regenerator matrix porous advection } \\
\text { transport coefficient }\end{array}$ & 0.95 & 0.95 & 0.95 & 0.95 & 0.95 & 0.95 \\
\hline
\end{tabular}

Notes:

a. Over the entire working fluid path, not just the heater. 


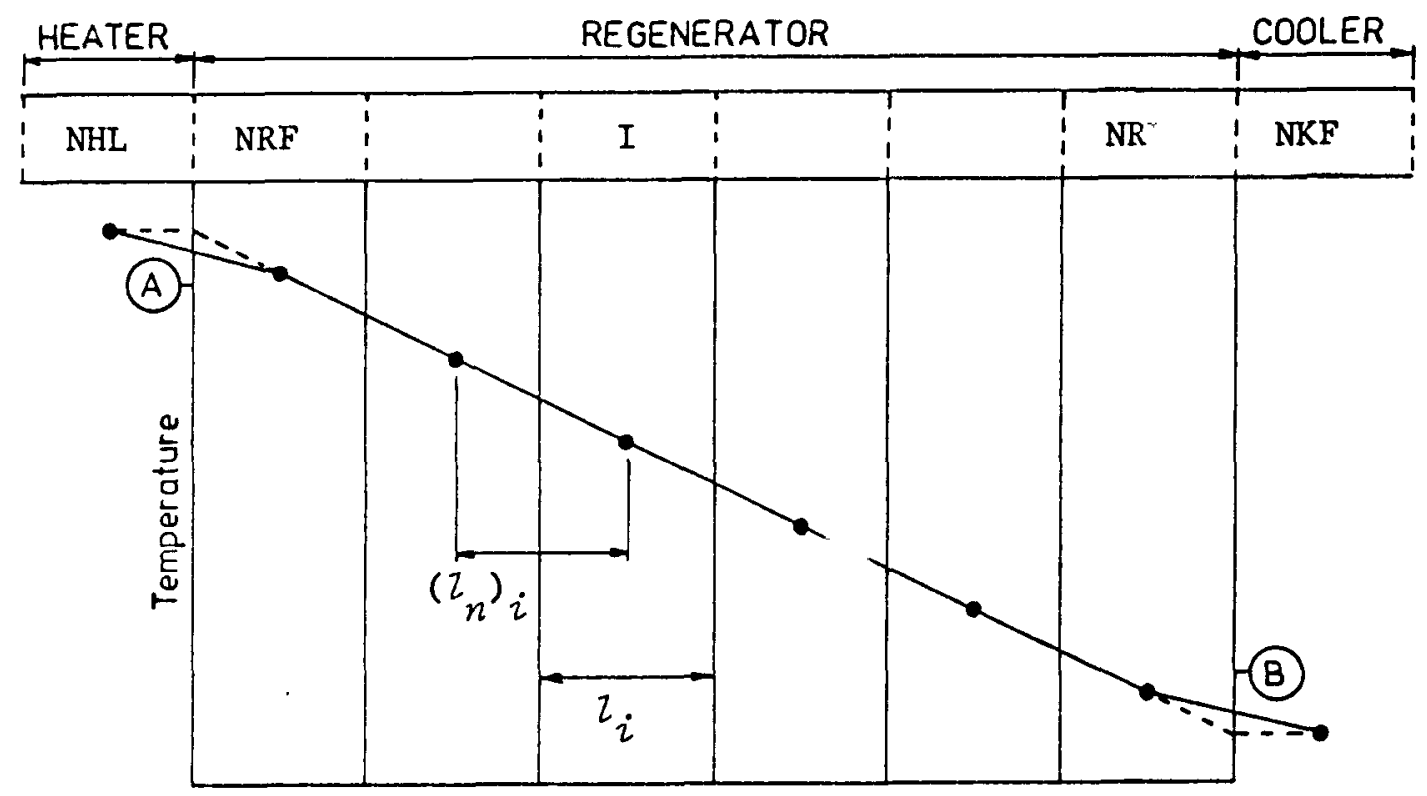

Figure 3.6 Regenerator enthalpy transport model.

if $\left(g_{n}\right)_{i} \geq 0$ then:

$$
\left(T_{n}\right)_{i} \approx T_{i-1}\left(1+0.5 K_{r}\left(1 / 1_{n}\right)_{i-1}\right)-0.5 K_{r}\left(1 / 1_{n}\right)_{i-1} T_{i-2}
$$

if $\left(g_{n}\right)_{i}<0$ then:

$$
\left(T_{n}\right)_{i} \approx T_{i}\left(1+0.5 K_{r} I_{i} /\left(I_{n}\right)_{i+1}\right)-0.5 K_{r}\left(I_{i} /\left(I_{n}\right)_{i+1}\right) T_{i+1}
$$

The upwind extrapolation format of these equations is necessary to maintain the transportive property of equations (2.55). The porous advection coefficient $K_{r}$ is introduced as a means of accounting for the deviation of actual regenerator behavior (for example, non-linear matrix temperature profiles and oscillating flow effects) from the ideal behavior suggested. Equations (3.4) apply at all the regenerator discrete momentum volume centroids (NRF to NRL+1) with the following exceptions:

if $\left(g_{n}\right)_{\mathrm{NRF}} \geq 0$ then: $\left(T_{n}\right)_{\mathrm{NRF}}-T_{\mathrm{NHL}}$

if $\left(g_{n}\right)_{\mathrm{NRL}+1}<0$ then: $\left(T_{n}\right)_{\mathrm{NRL}+1}-T_{\mathrm{NKF}}$

It may be noted that at discrete momentum volume centroid $N R F+1$, the adjacent heater temperature would be used for $T_{i=2}$ in equation (3.4.1). Similarly, at discrete momentum volume centroid NRL, $T_{\mathrm{NKF}}$ is substituted for $T_{i+1}$ in equation (3.4.2). This is felt to be physically more consistent than the alternative of assuming that $T_{\mathrm{NHL}}$ occurs at $\mathrm{A}$ and $T_{\mathrm{NKF}}$ occurs at $\mathrm{B}$ in figure 3.6 . In practice though, the difference between the two approaches is minimal. 
Owing to the magnitude of the maximum Reynolds numbers occurring over the flow field (table 3.3) in the SPDE, it was found necessary to set $k_{r}$ almost at unity to obtain agreement with the experimental heat transfer data. However, it is important to note in the context of comparing the one- and twodimensional heater module simulations that no modifications are made to the nominal Kays and London empirical correlations in the heater.

The maximum Mach and Reynolds numbers are similar for all three simulations. The smallness of the Mach numbers attests to the absence of any choking while the magnitude of the Reynolds numbers is indicative of the level of flow turbulence. Of particular importance are the low characteristic numbers $\left(N_{C h}\right)$ of 24 to 26 (compared, for example, with a $N_{C h}$ range of 60 to 250, depending on operating parameters, for the GM-GPU3 engine (Go87)). This indicates that with only about 25 complete information traverses per cycle, ignoring information propagation effects in a simulation may not be automatically justified. This is borne out by the comparison of the experimental and simulation data given in table 3.4 for. the two experimental test points.

Generally, the one-dimensional UPD simulation and experimental results are in agreement. The maximum energy balance discrepancy is less than 5.58 (external heat supply for test 42) while the simulated and measured expansion and compression space mean cyclic temperature and pressure parameters are in reasonable agreement. In contrast, the one-dimensional equilibrium simulation shows an overall discrepancy of about 308 in the indicated piston work. The major source of this error is the mismatch between the measured and simulated compression space pressure profile phase angles (which are given relative to the piston displacement). Since the indicated wor:: is proportional to the sine of the phase angle, a $1.2^{\circ}$ discrepancy makes a 198 contribution towards the indicated piston work discrepancy. It should be noted that no energy balance errors are reported for the simulations owing to a data output processing error discovered in the code. This error was discovered and corrected during the METR simulation runs reported in chapter 5 . The corrected code typically yields energy balance errors of the order of .018.

Hence it is possible that the indicated work discrepancy between the equilibrium and UPD algorithms is a consequence of information propagation effects. This is suggested by the relative agreement between all the remaining experimental and one-dimensional equilibrium simulation parameters with the exception of the mean compression space temperature. This is higher in the simulation because of the larger predicted pressure profile phase angle. However, the overall energy balance simulation results produced by the equilibrium algorithm (for test 46 at least) apparently agree with those produced by other simulation codes ( $\mathrm{Te} 88$ ) such as the Gedeon GLIMPS (Ge86) and NASA Lewis SNAP (Te83) codes. In contrast, the MTI harmonic analysis code appears to conform to the UPD algorithm predictions ( $\mathrm{Te} 88$ ). However, this superficia" inter-code comparison is probably only of anecdotal significance because, in this context, a detailed irreversibility comparison at least is necessary in order to understand how well the codes compare with each other. Unfortunately, such data is not yet available. 
Table 3.4 Experimental/simulation data comparison for the one- and two-dimensional heater modules.

SPDE Test $46(3 / 14 / 86)$

\begin{tabular}{cccc}
\hline Experi- & 1-Dim. & 1-Dim. & 2-Dim. \\
mental $^{2}$ & Heater: & Heater: & Heater: \\
& UPD & Equil. & Equil. \\
& Algor. & Algor. & Algor.
\end{tabular}

External heat supplied (J)

External heat rejected (J)

Piston indicated work $(J)$

Energy balence error (\%)

Indicated efficiency based

on piston ind. power (\%)

$\begin{array}{ccccccccc}295.11 & 295.41 & 295.2 & 303.02 & 460.86 & 436.78 & 437.66 & 453.74 \\ 242.56 & 244.18 & 220.84 & 219.66 & 337.88 & 349.61 & 316.22 & 315.27 \\ 57.546 & 56.910 & 75.34 & 75.48 & 89.654 & 90.809 & 119.69 & 118.35 \\ 3.4920 & -b & -6 & -6 & 12.006 & -b & -6 & -6 \\ 19.5 & 19.3 & 25.5 & 24.6 & 19.5 & 20.8 & 27.4 & 26.1\end{array}$

SPDE Test $42(9 / 11 / 86)$

\begin{tabular}{cccc}
\hline $\begin{array}{c}\text { Experi- } \\
\text { mental }\end{array}$ & $\begin{array}{c}\text { 1-Dim. } \\
\text { Heater: } \\
\text { UPD } \\
\text { Algor. }\end{array}$ & $\begin{array}{c}\text { 1-Dim. } \\
\text { Heater: } \\
\text { Equil. } \\
\text { Algor. }\end{array}$ & $\begin{array}{c}\text { 2-Dim. } \\
\text { Heater: } \\
\text { Equil. } \\
\text { Algor. }\end{array}$ \\
\hline 460.86 & 436.78 & 437.66 & 453.74 \\
337.88 & 349.61 & 316.22 & 315.27 \\
89.654 & 90.809 & 119.69 & 118.35 \\
12.006 & -6 & -6 & .6 \\
19.5 & 20.8 & 27.4 & 26.1 \\
& & & \\
\hline
\end{tabular}

\begin{tabular}{lllllll}
\hline $\begin{array}{l}\text { External heat supply } \\
\text { discrepancy }(x)\end{array}$ & - & 0.10 & 0.03 & 2.68 & -1.55
\end{tabular}

External heat rejection

discrepancy $(x)$

Piston indicated work

discrepancy $(x)$

\begin{tabular}{|c|c|c|c|c|c|c|c|c|}
\hline Mean exp. space temp. $\left({ }^{\circ} \mathrm{C}\right)$ & 296.83 & 291.79 & 296.08 & 289.20 & 404.69 & 390.88 & 395.69 & 387.00 \\
\hline Mean comp. space temp. $\left({ }^{\circ} \mathrm{C}\right)$ & 23.708 & $23.408^{c}$ & $30.325^{c}$ & $30.94^{\mathrm{C}}$ & 55.908 & $56.225^{\circ}$ & $64.82^{c}$ & $64.77^{6}$ \\
\hline $\begin{array}{l}\text { Comp. space pressure } \\
\text { ampl itude (bar) }\end{array}$ & 11.803 & $12.420^{c}$ & $12.468^{c}$ & $12.459^{\circ}$ & 15.472 & $16.526^{\mathrm{c}}$ & $16.496^{\mathrm{c}}$ & $16.389^{c}$ \\
\hline $\begin{array}{l}\text { Comp. space pressure } \\
\text { angle (deg) }\end{array}$ & 7.8233 & 7.5 & 9.0 & 9.0 & 7.0510 & 6.9 & 9.0 & 9.0 \\
\hline
\end{tabular}

Notes:

a. Taken to be the mean of the left- and right-hand engine test results.

b. Calculated energy balance errors are too large owing to a data output processing error; errors produced by corrected code are about .01\% at most (see table 5.3).

c. Mean of peripheral and central lower compression space values. 
In the face of this dilemma, several other suggestions have been made to explain the inaccuracy of the equilibrium algorithm, for example, gas leakage between the expansion and compression spaces which is not explicitly modelled. In particular, if the inclusion of gas leakage were to eliminate the equilibrium algorithm error, then it may be concluded that information propagation is not important and that the apparent accuracy of the UPD algorithm is a spurious numerical effect.

In the light of the uncertainty about the information propagation issue and the apparent agreement between the equilibrium algorithm, GLIMPS and NASA codes for the SPDE, NASA and the principal investigator felt that the twodimensional module simulation should proceed using the equilibrium algorithm only pending further work on the information propagation issue, which is discussed in chapter 4.

The predictions of the equilibrium simulations for the one- and twodimensional heaters are similar, at least from an overall cyclic performance perspective. For test 46, the simulated external heat supplied using twodimensional heater module is 2.68 larger than that predicted using the onedimensional heater module (with the latter value being within .038 of the measured external heat supply). In contrast, for test 42 , the two-dimensional simulated external heat supplied is in closer agreement with the measured value than the corresponding one-dimensional prediction. The expansion space mean cyclic temperature (which is strongly influenced by the heat supplied in the heater) does not exhibit contradictory behavior since, for both tests, the two-dimensional simulated value is less than the one-dimensional simulated value which in turn is less than the experimental value.

In this light, a more detailed view of the influence of two-dimensional effects may be discussed in terms of figures 3.7 to 3.10 . Figures 3.7 and 3.8 show the cumulative heater wall/fluid heat transfer as a function of angle for tests 46 and 42 respectively while figures 3.9 and 3.10 show the volumeaveraged midpoint heater fluid temperature profiles. In all cases, the onedimensional heater UPD and equilibrium algorithm profiles are in close agreement. The two-dimensional heater heat transfer profiles reflect lower cumulative heat transfers throughout the cycle, converging rapidly towards and then exceeding the one-dimensional profiles beyond $320^{\circ}$. The two-dimensional volume-averaged heater midpoint temperatures are less than their onedimensional counterparts within a range of about 5 to $10 \mathrm{~K}$ over the cycle. Hence it is evident that the mechanism of heat transfer simulated in the onedimensional heater using empirical correlations is different from that simulated in the two-dimensional heater without such empiricism. In the light of the high cyclic Reynolds numbers and resultant turbulence, the numerical discrepancy is an expected result since the two-dimensional simulation assumes laminar flow in the heater. Thus inclusion of a turbulence model in the heater should at least reduce the instantaneous numerical discrepancy, although the extent of the narrowing depends not only on the efficacy of 


\section{HEATER WALL/FLUID HEAT TRANSFER}

\section{SPDE TEST 46 OF 3/14/86}

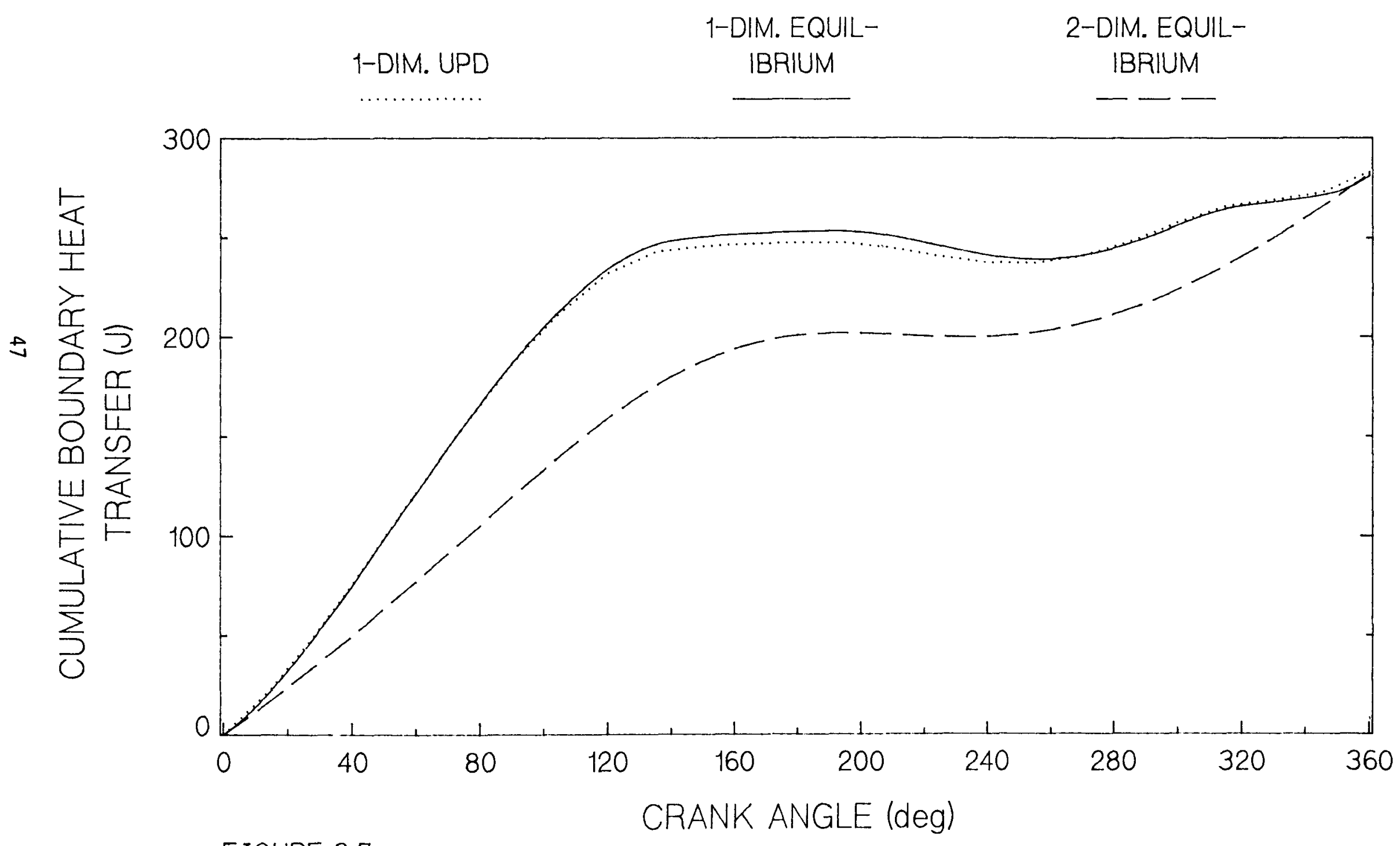

FIGURE 3.7 


\section{HEATER WALL/FLUID HEAT TRANSFER}

SPDE TEST 42 OF 9/11/86

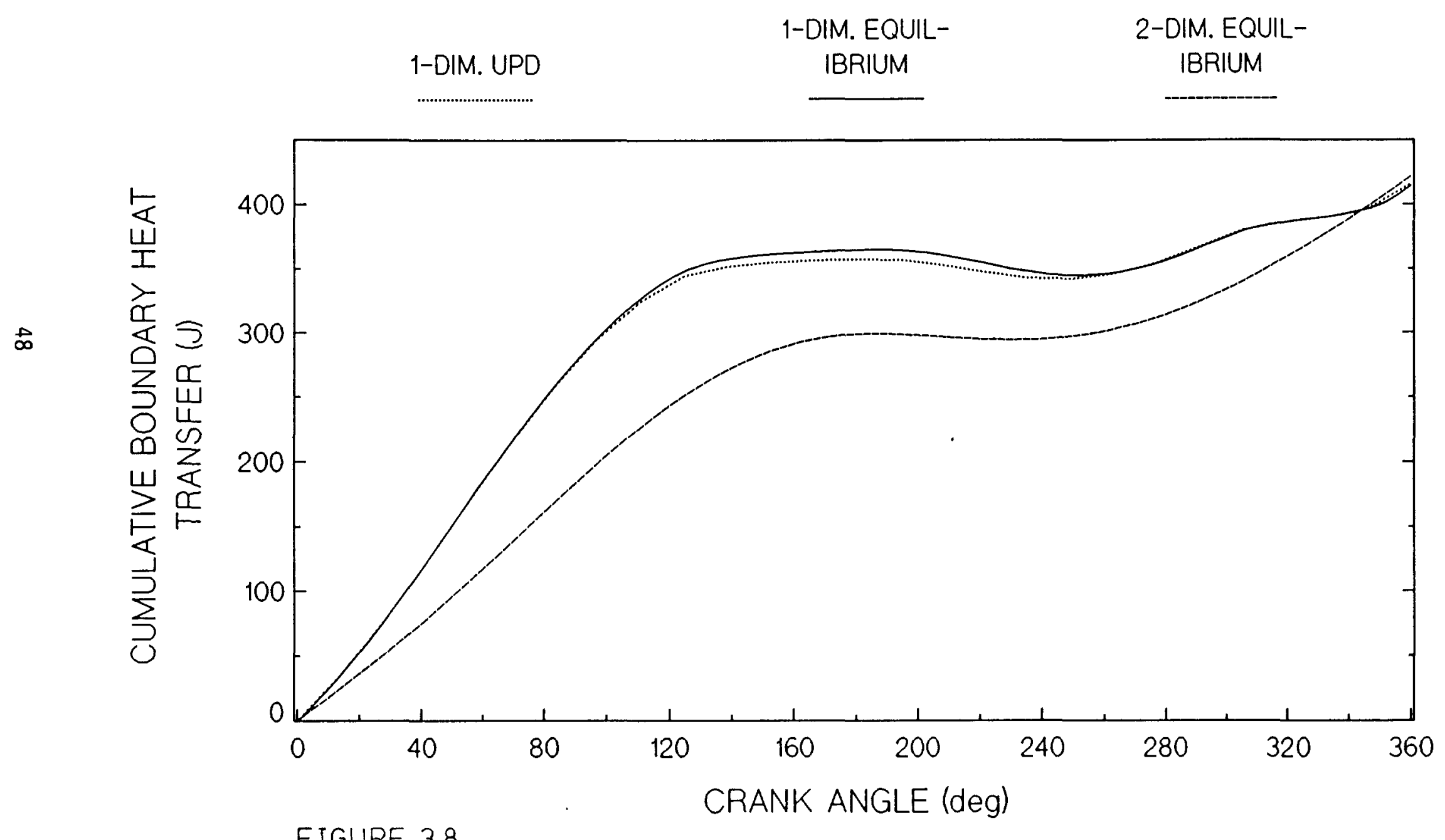

FIGURE 3.8 


\section{HEATER MIDPOINT TEMPERATURE}

SPDE TEST 46 OF 3/14/86

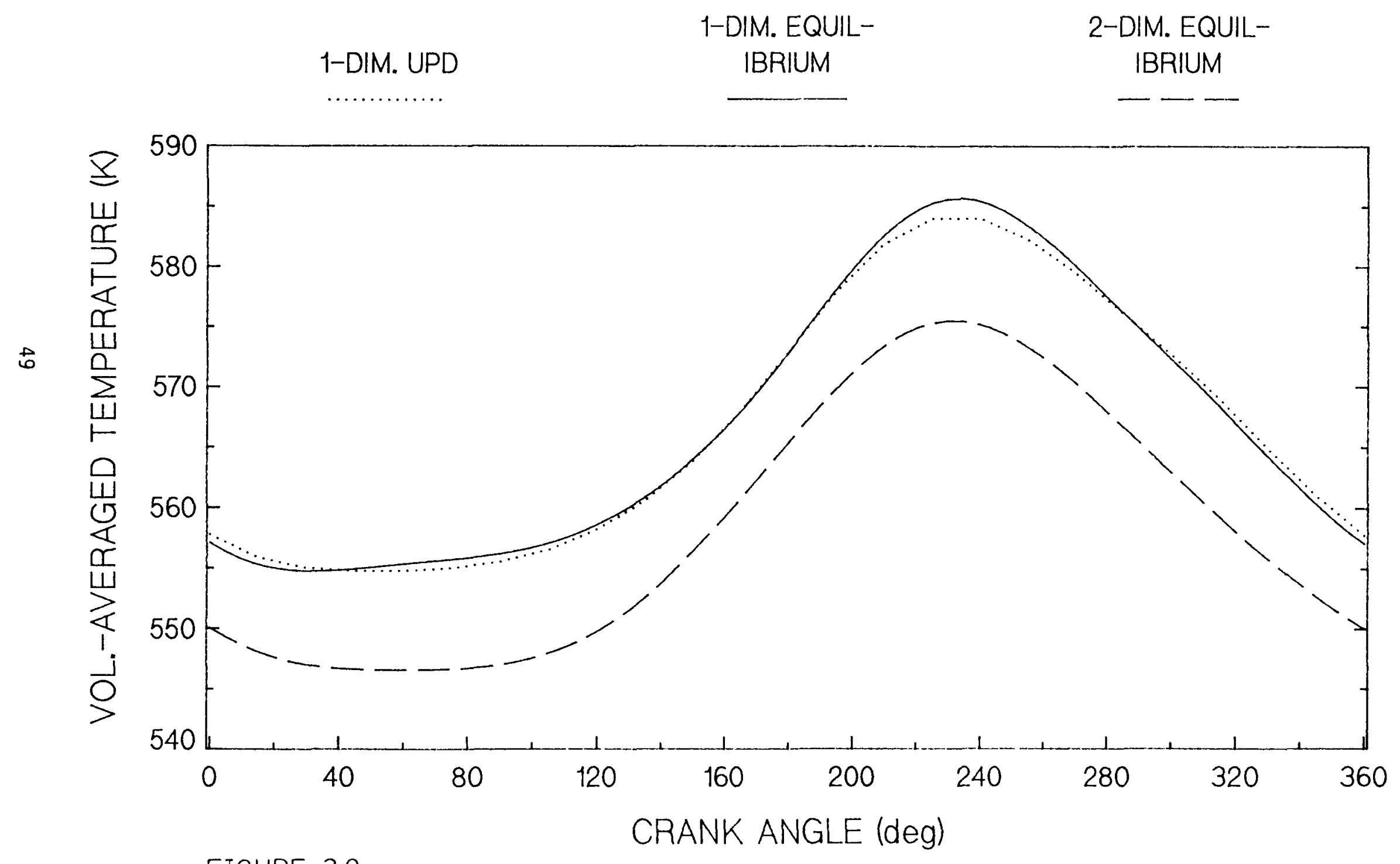

FIGURE 3.9 


\section{HEATER MIDPOINT TEMPERATURE}

SPDE TEST 42 OF 9/11/86

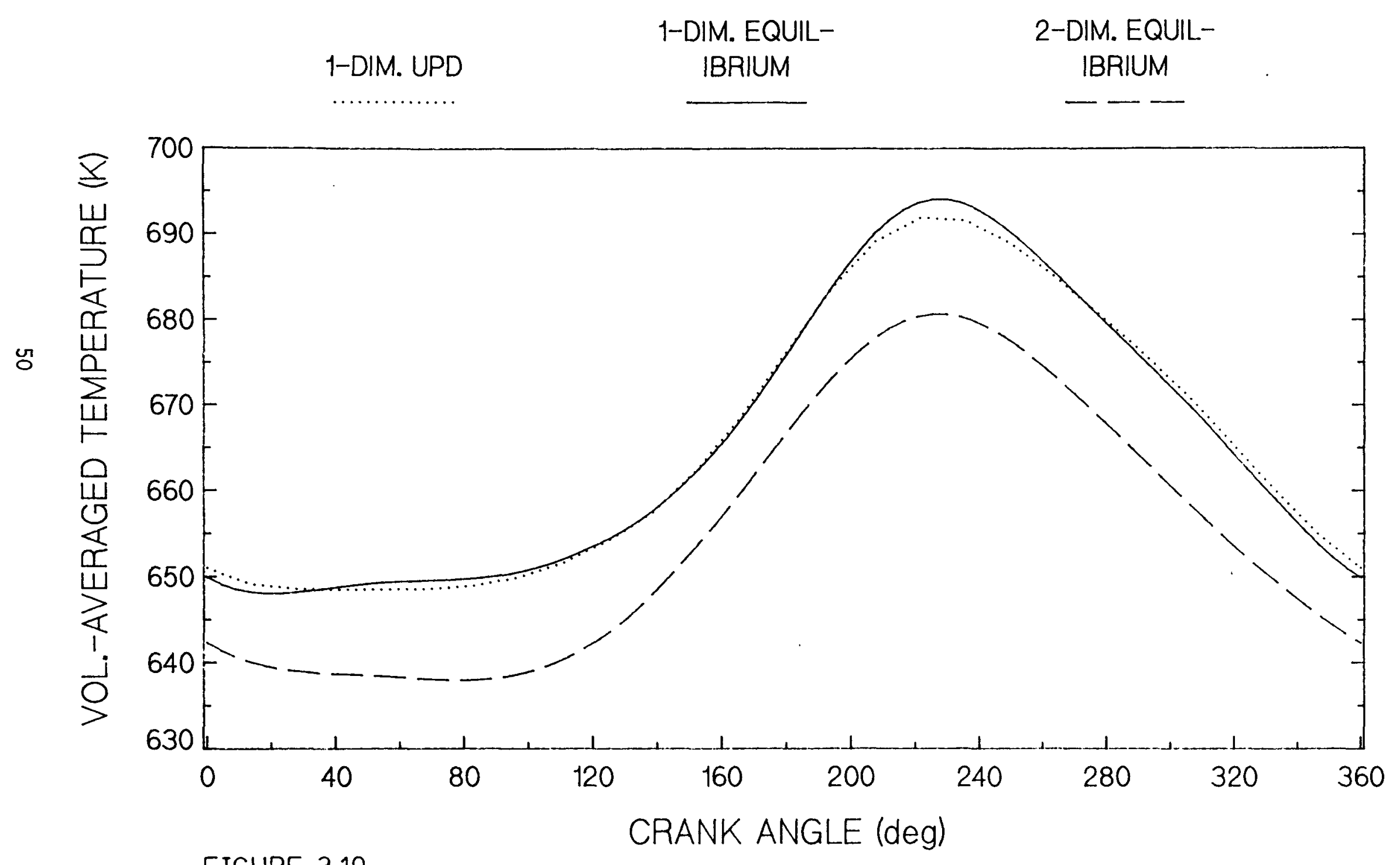

FIGURE 3.10 
the turbulence model but also on the errors inherent in using a steady-state correlation. These issues can only be satisfactorily addressed by an experimentally based investigation such as that of the METR (see chapter 5). However, it must be noted that from an overall or systems cyclic energy balance perspective, the one- and two-dimensional heater module simulations do not show any appreciable differences. This emphasizes the limitations of inferring the validity of a simulation code purely from time-averaged parameter and cyclic energy balance data. Apparent simulation validation based on such criteria may be a result of error cancellation effects rather than an indication of actual 'e accuracy.

The aggregate system velocıly fields produced for test 42 for the oneand two-dimensional heater modules are depicted in figures 3.11 and 3.12 . In each case, the cyclic angle is plotted on the $X$ axis while the location along the one-dimensional working fluid path is denoted by the $Y$ axis. In terms of the sign convention of figure 3.1, the expansion space is located on the left $(Y \approx 0)$ and the lower compression space on the right, the two spaces being separated by the heater, regenerator, cooler, upper compression space and conical connecting passage. The dashed lines indicate negative velocities. Figures 3.11 and 3.12 exhibit no qualitative differences and are similar in shape. Quantitatively, there are small differences between the minimum and maximum velocities amounting to $3 \%$ at most. The test 42 one- and twodimensional heater module simulation aggregated temperature fields shown in figures 3.13 and 3.14 exhibit comparative behavior similar to that of the velocity fields. The notable, yet consistent, exception is that the maximum temperature in the one-dimensional heater module is $9 \mathrm{~K}$ greater than that in the two-dimensional heater module as shown in figure 3.10 .

These aggregate system parameter profiles again show that no major qualitative differences are introduced into the simulation by using a twodimensional heater module even on a transient basis when assuming laminar flow in the heater. Quantitative effects are also relatively small and limited to the heater itself. Therefore, it seems reasonable to suppose that systemimposed boundary conditions on the two-dimensional heater module force the two-dimensional flow in the module to conform on aggregate to that simulated using a one-dimensional heater module. This in turn suggests that twodimensional component simulation may not be an effective way of improving the system accuracy of stirling machine simulation. Such improved accuracy is probably only realisable by simulating most (if not all) of the working fluid path in two-dimensions. Details of the two-dimensional flow field in the aggregated heater tube are not presented here since they do not bear directly upon the systems nature of this discussion. It is sufficient to note that these profiles are in agreement with laminar oscillating flow results published in the literature. Details of the two-dimensional flow field are presented in chapter 5 where they are evaluated against experimental data produced by the METR. 


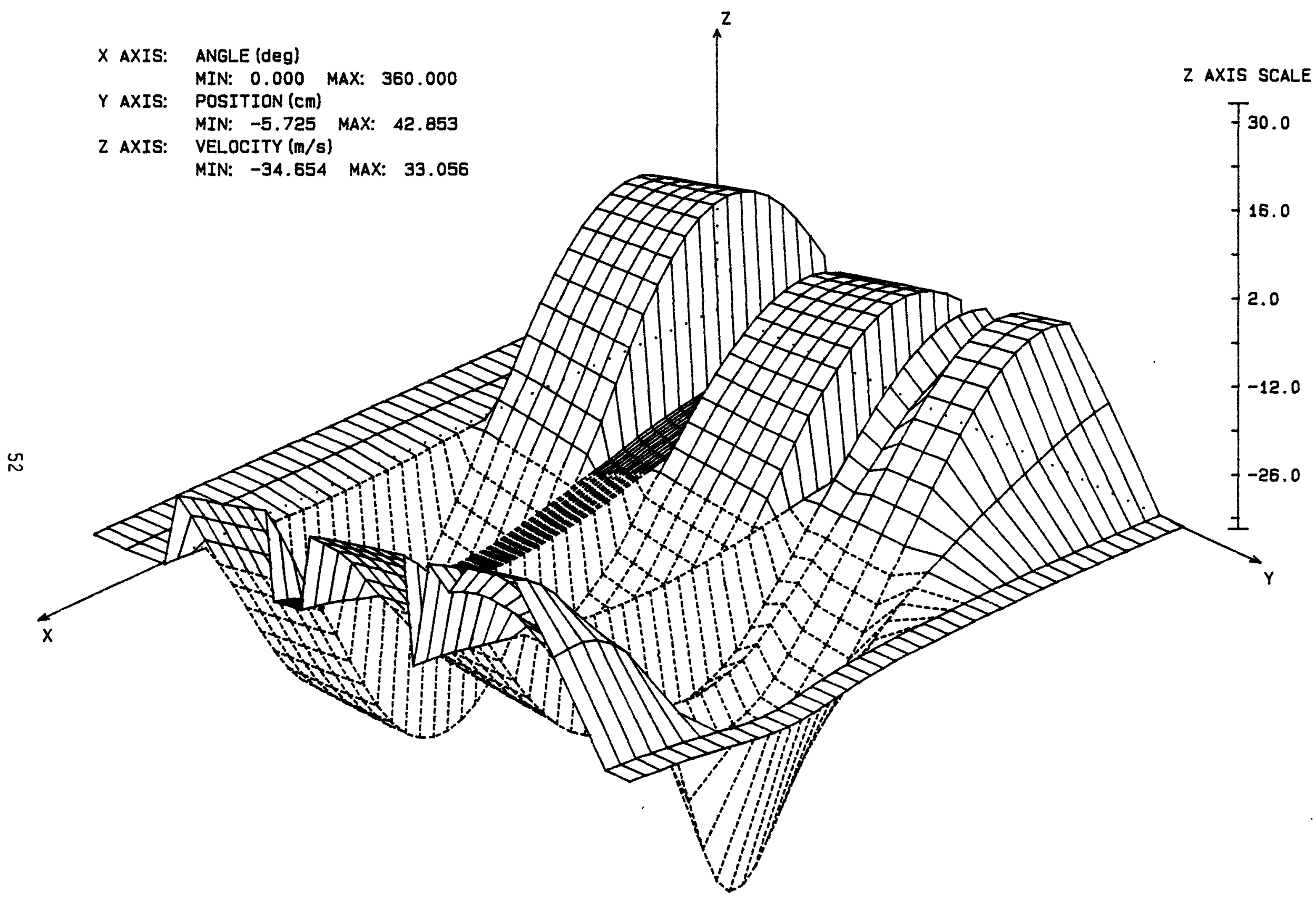

Figure 3.11 SPDE velocity field (equilibrium algorithm. 1-dim. heater) 


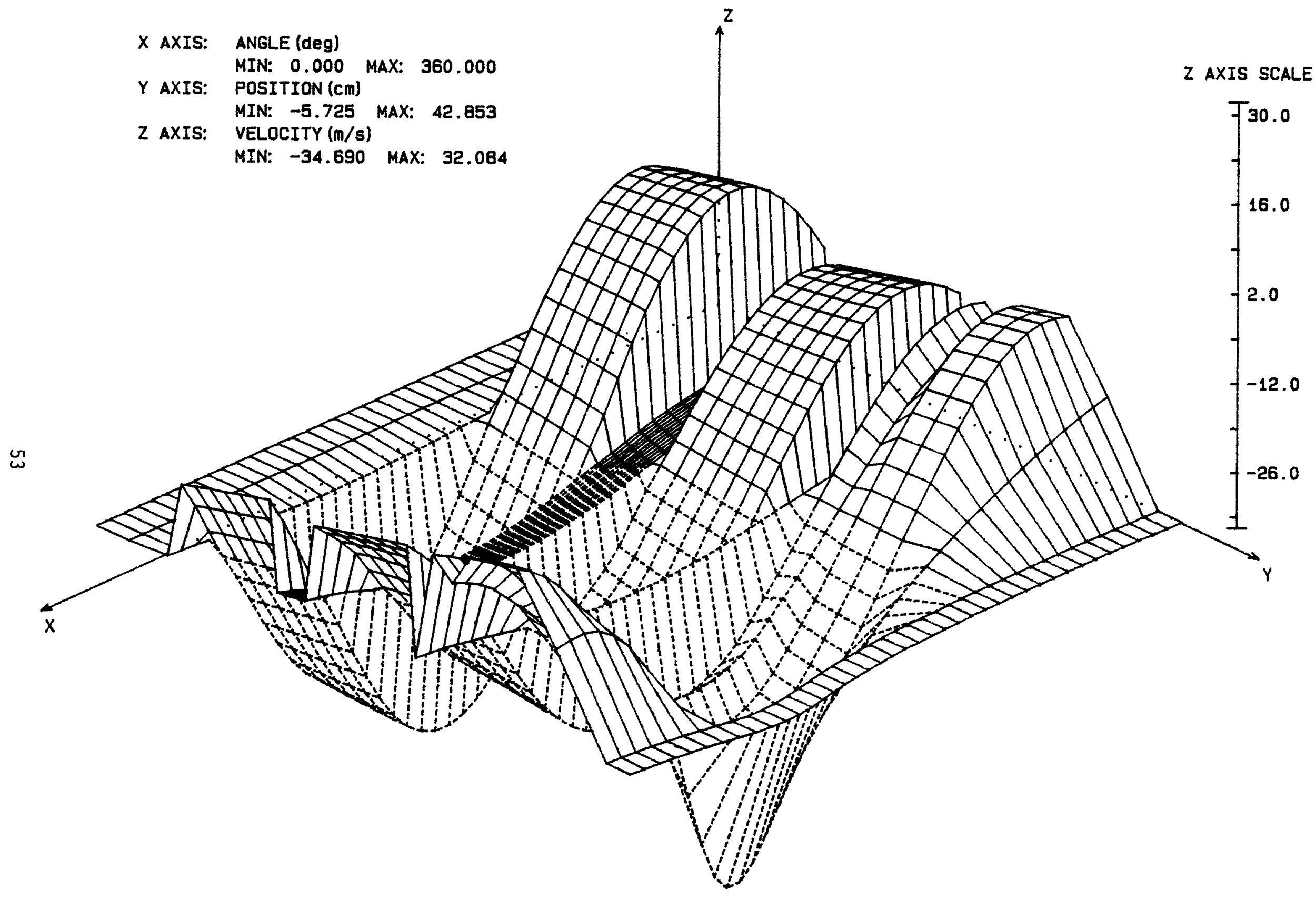

Figure 3.12 SPDE velocity field (equilibrium algorithm, 2-dim. heater) 


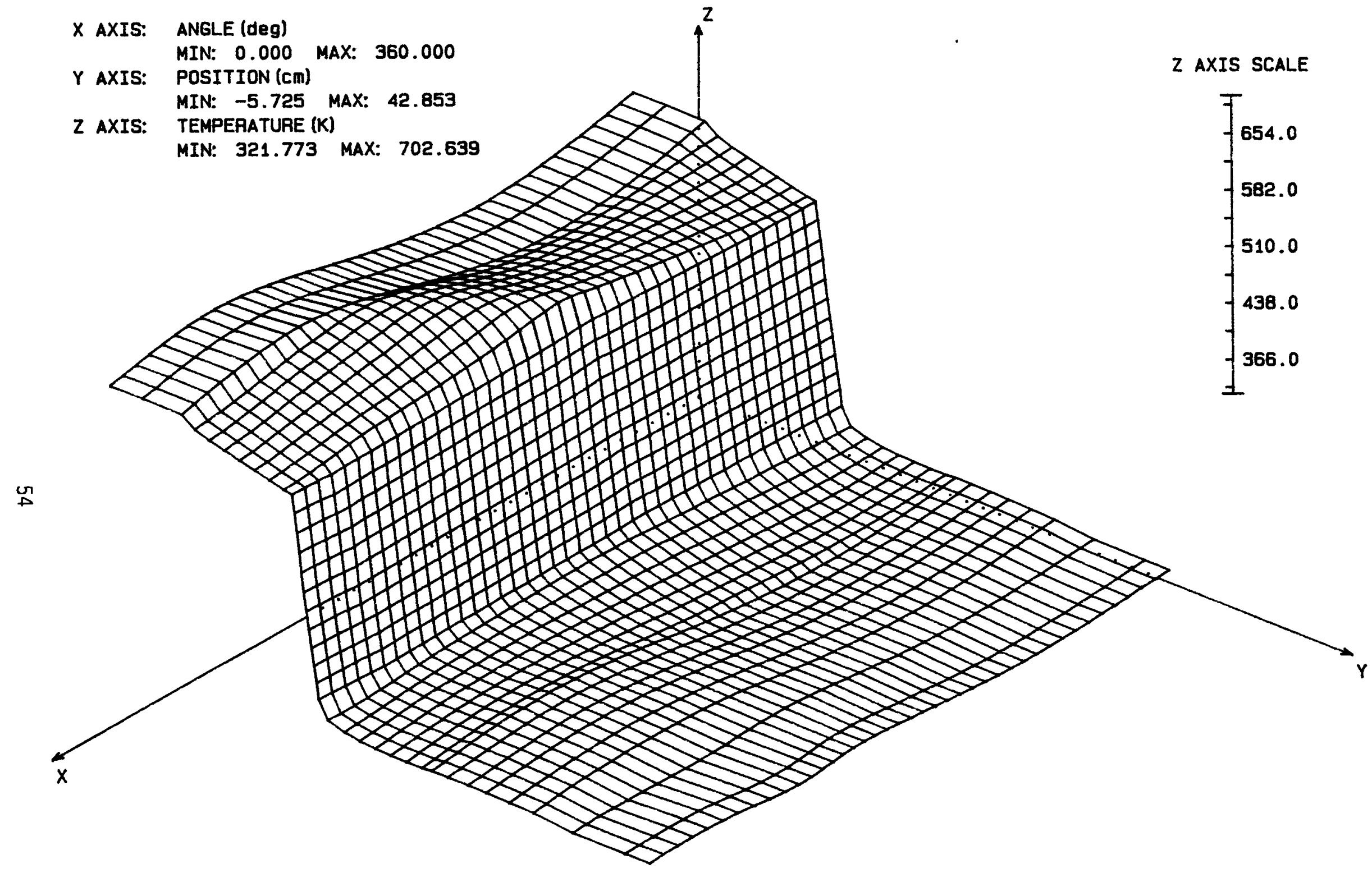

Figure 3.13 SPDE temperature field (equilibrium algorithm, 1-dim. heater) 


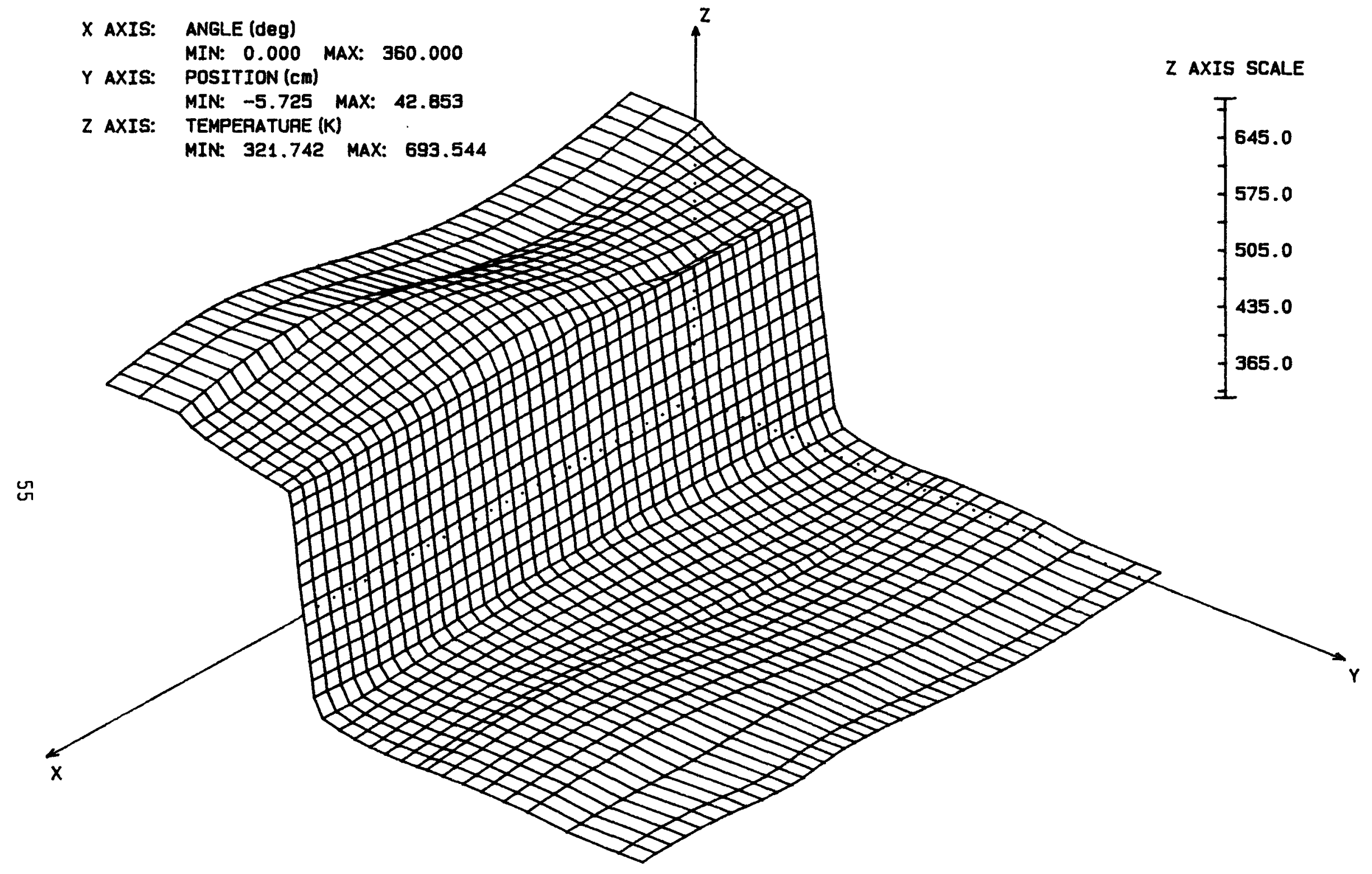

Figure 3.14 SPDE temperature field (equilibrium algorithm, 2-dim. heater) 


\subsection{CLOSURE}

The baseline comparison of the one- and two-dimensional heater module simulated and experimental data shows that two-dimensional flow effects do impact the transient heat transfer predictions in the heater itself. However, these effects are not proportionately manifest on the system level where they only minimally affect the cyclic energy balance. Turbulence effects are likely to be the major cause of the lack of quantitative correspondence between the simulated flows in the one- and two-dimensional heater modules since the former nominally includes the impact of turbulence via heat transfer coefficient and friction factor correlations. While this justifies isolated component studies of oscillating turbulent flow, it does not alter the contention that an overall improvement in Stirling machine simulation accuracy probably is achievable only if a major portion of the fluid flow path (at least the heater, regenerator, and cooler assembly) is simulated in twodimensions.

The information propagation issue has been established as a possible performance issue in SPDE class (high frequency, high pressurisation) Stirling engines. This subject is by no means new to stirling machine simulation and has been dealt with by others (such as Organ (Or82) and Taylor (Ta84)) although chiefly in the context of method of characteristics simulations. The information propagation issue has engendered much contention among Stirling machine analysts with some suggesting that it is not physically relevant. If the results produced here serve no other purpose than to stimulate debate and definitive research to resolve the information propagation issue, they will have served NASA well. 


\subsection{INTRODUCTION}

One of the conclusions from the simulation of the SPDE has been that, apparently, an accounting of information propagation effects permits convergence between the simulated and measured cyclic energy performance parameters to be achieved. In particular, the simulated piston indicated work can be made to agree with its measured counterpart only when the Unitary Pressure Domain (UPD) algorithm is used. Invocation of the equilibrium algorithm consistently leads to an over-prediction of the piston indicated work. This over-prediction is principally related to the phase angle between the piston displacement and compression space pressure profiles. The equilibrium algorithm produces an over-estimate of the phase angle which, although small in absolute terms ( 1 to 2 degrees), is significant because the indicated work is proportional to the sine of the phase angle. This is the major contributor towards the observed simulation/experimental discrepancies of about 308 .

The following postulates may be used to explain the apparent fidelity of the UPD algorithm:

- The information propagation effect is physically significant and is being portrayed accurately by the UPD algorithm.

- The information propagation effect is physically significant and its portrayal by the UPD algorithm is a spurious numerical effect.

- Information propagation is not significant and the apparent accuracy of the UPD algorithm is fortuitous.

A feasible means of testing these postulates is to apply the UPD and equilibrium algorithms to a problem that has a well-defined and validated closed-form, analytic solution. Ideally, this problem should closely correspond with the boundary conditions prevailing in Stirling machines in general and in the SPDE in particular.

The process adopted has been to apply the existing UPD and equilibrium algorithms as used in the one-dimensional SPDE simulation to the selected problem. This enables an assessment of whether information propagation effects offer a physically reasonable explanation for the observed behavior of the algorithms when applied to the SPDE. Thereafter, the numerical accuracy of the algorithms is assessed in order to investigate whether spurious numerical effects are occurring. 


\subsection{PROBLEM SELECTION}

A general class of analytically soluble problems involving information propagation effects revolves around the description of acoustic transmission phenomena in plain tubes. A classic treatise on these problems by Lord Rayleigh (St26) encompasses analytic solutions including non-1inear effects such as viscous dissipation and finite boundary heat transfer.

Using Rayleigh's development of Kirchoff's equations of sound, Iberall (Ib50) developed a first order analytic solution for the transmission line problem. This problem is defined geometrically by a length of tube connecting an infinite cavity with a rigid receiver volume. The analysis seeks to define the phase lag and amplitude ratio of the pressure profile in the receiver volume with respect to a sinusoidal pressure variation within the cavity. Physically, the problem is representative of a transmission line connecting a signal source to a pressure transducer. The desired outcome of the analysis is a means of providing design guidelines on the length and diameter of the transmission line so as to ensure adequate measurement accuracy of the pressure transducer.

In another investigation, Chester (Ch64) analyzed the behavior of resonant oscillations in closed tubes. The prescribed boundary conditions are that one end of the tube is closed while the other is excited by a piston oscillating at near-resonant frequencies. The objective of the analysis is to investigate the impact of compressive viscosity and boundary shear viscosity on the gas oscillations in a well-defined frequency band around resonance in which shock waves occur.

In another analysis, Jimenez (Ji73) extends Chester's analysis to a case in which the closed end of the tube is replaced with an arbitrary closure condition ranging from fully open to fully closed. By expanding the momentum equations in terms of a Mach number series, both the amplitude and form of the oscillations are predicted in order to show that in both the fully open and fully closed cases, shock waves are needed to describe the observed resonant behavior.

Although the problem described by Chester and Jimenez is physically more in conformity with the SPDE geometry, their analyses do not correspond with the general flow situation prevailing in Stirling machines, particularly owing to their appropriate neglect of heat transfer effects. Furthermore, these analyses are focussed on resonant effects rather than on conditions far from resonance where the classical linearised theory is assumed to prevail. Hence, despite its lack of boundary condition conformity with Stirling machines, Iberall's analysis is preferred as a means of investigating the validity of the aforementioned postulates. This is justified by the inclusion of a more complete fluid dynamic treatment in the analysis and its yield of information more amenable to intuitive interpretation because of the practical engineering relevance of the transmission line problem. Furthermore, several experimental validations of Iberall's analysis have been performed (Wa65, Go68) which lend credence to using the analysis as a benchmark against which the simulation may be compared. 
The geometry of the transmission line is shown in figure 4.1.

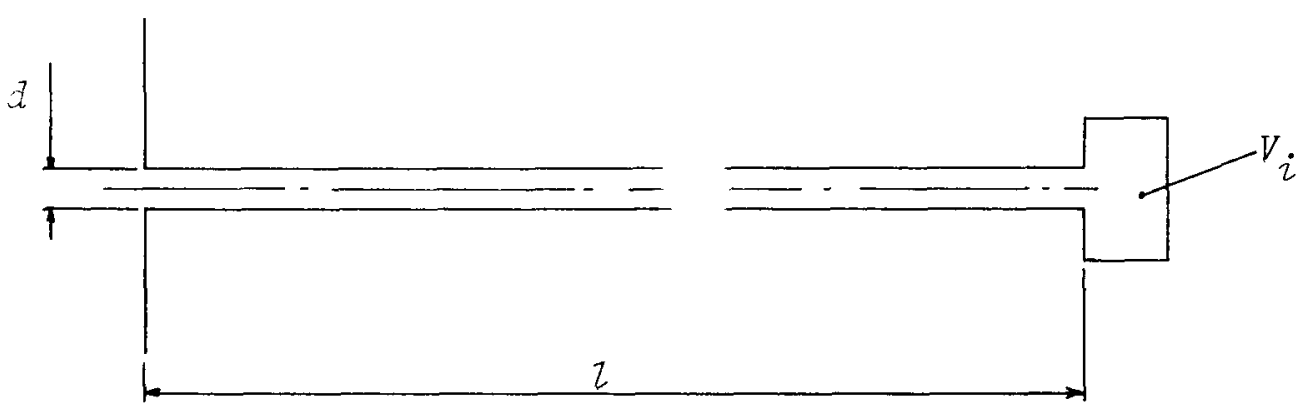

Figure 4.1 Transmission line geometry.

A tube of length 1 and diameter $d$ is connected between a cavity and an instrument volume $V_{i}$. The following assumptions that are significant to the comparison exercise are made:

1. The walls of the instrument volume are flexible so that the actual volume may be replaced by a larger equivalent volume that will store the same mass of fluid per unit of pressure change.

2. The flow is laminar throughout the tube.

3. The gas expands and contracts isothermally in the instrument cavity.

4. The excitation oscillatory pressure is sinusoidal.

Within these constraints, the following factors are taken into account in the analysis:

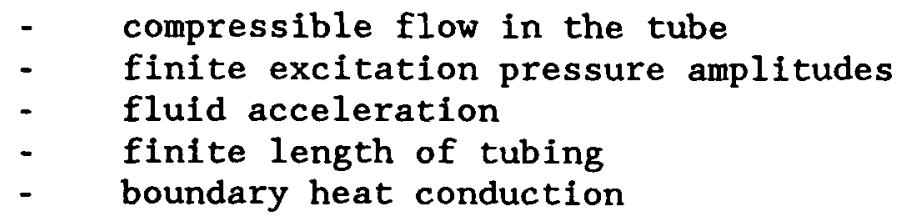

The analytic solution is represented by equation (116) (not repeated here) in Ib50. Owing to its complexity, the equation may be conveniently solved via a computer program. A key part of the solution involves the computation of Bessel functions. In the computerized implementation of the analysis offered by Watts (Wa65), a normal series solution is used for kinetic Reynolds (or Valensi) numbers less than 200, while an asymptotic series solution is used otherwise. Goldschmied (Go68), however, apparently uses a normal series solution throughout. In the implementation used here, Watts' 
approach is preferred due to its demonstrated superior numerical accuracy. Howevex, while Watts performs the calculations in real arithmetic, complex arithmetic is used throughout here (following Goldschmied) in order to avoid what appears to be a coding inconsistency in Watts' implementation.

In both Watts' and Goldschmied's implementations, no details of the method used to determine the excitation pressure amplitude are given. As the amplitude is a critical parameter from a simulation point of view, the methodology adopted here has been to choose approximately the largest amplitude that produces simulated laminar flow throughout the tube (Reynolds numbers less than 2000). Under certain conditions (particularly at higher frequencies) this still yields excitation pressure amplitudes that are very small in comparison with the mean pressure. This produces some unavoidable truncation errors in the simulation, which although minimized through the use of double precision arithmetic, must be borne in mind when interpreting the results produced.

\subsection{THE SIMULATION MODEL}

The standard SPDE simulation programs have been applied without alteration to the geometry defining a transmission line. The codes have been modified to accept a pressure profile and a rigid cavity as boundary conditions which replace the piston/cylinder boundaries of the SPDE codes. The fluid dynamics of the instrument cavity are modelled in full so that the isothermal assumption made in Iberall's analysis is not replicated by the simulation. The standard Kays and London (KL64) friction factor and heat transfer coefficient correlations used in the SPDE simulations are applied without any empirical corrections. Care has been taken to ensure that both Iberall's analysis and the simulation use identically the same thermodynamic initial conditions. This has required some reworking of the constants in Iberall's analysis to reflect the use of temperature rather than density as an initial condition specifier.

In view of the varying temporal resolution produced by the UPD and equilibrium algorithms, extracting amplitude and phase information from the simulated pressure profiles can be subject to large errors, particularly at low characteristic numbers (less than about 20). Several approaches have been tested including sine transforms, sine quadratures and globally implicit cubic spline fits. The last approach has produced the most reliable results and has therefore been adopted. At each data point, the known excitation amplitude and phase angle have been used to check the integrity of the cubic spline fits.

\subsection{APPLICATIONS}

Two case studies have been performed to compare the analytical and simulation results. The first geometry corresponds to that used by Watts (Wa65) to experimentally validate Iberall's analysis. This geometry is preferred to that of Goldschmied (Go68) in view of the good agreement of 
Watts' experimental data with that calculated, especially under conditions of resonance.

The second geometry represents a crude approximation of the SPDE in which the flow passage between the expansion and compression spaces is modelled as a length of tube with a diameter equal to the average of the heater and cooler tube diameters. The end effects of the variable volume spaces are neglected so that the tube termination geometry is identical to that used in Watts' experiment.

\subsubsection{Watt' Experimental Rig}

The invariant parameters used to describe the geometry and initial conditions of the transmission line are listed in table 4.1. These parameters correspond with those given in Wa65 to describe the 4-ft transmission line tested. The additional input parameters required, namely, the excitation pressure amplitude and frequency, are treated as variables.

Table 4.1 Transmission line parameters.

\begin{tabular}{ll}
\hline Transmission line length (mm) & 1220 \\
Transmission line internal diameter(mm) & 7.818 \\
Instrument cavity diameter (mm) & 14 \\
Instrument cavity volume (cm^3) & .414 \\
Working fluid & Air \\
Mean excitation pressure (bar) & .785 \\
Mean system temperature (deg C) & 25.745
\end{tabular}

Using a constant excitation pressure amplitude of 0.016 bars, Iberall's analysis yields the results plotted in figures $4.2,4.3$, and 4.4. Figure 4.2 shows the exponential decrease in characteristic number as a function of excitation frequency. Figure 4.3 depicts the variation in amplitude ratio (that is, the ratio of the instrument cavity pressure profile amplitude to that of the excitation pressure profile) with excitation frequency. Figure 4.4 shows the corresponding variation in the phase lag of the instrument cavity pressure profile to that of the excitation pressure. The amplitude ratio profile replicates that reported by Watts and hence shares the experimental validity of Watts' data (Watts does not report any phase lag comparison data). The data span an excitation frequency range centered upon 
TRANSMISSION LINE: IBERALL'S ANALYSIS

CHARACTERISTIC NUMBER PROFILE

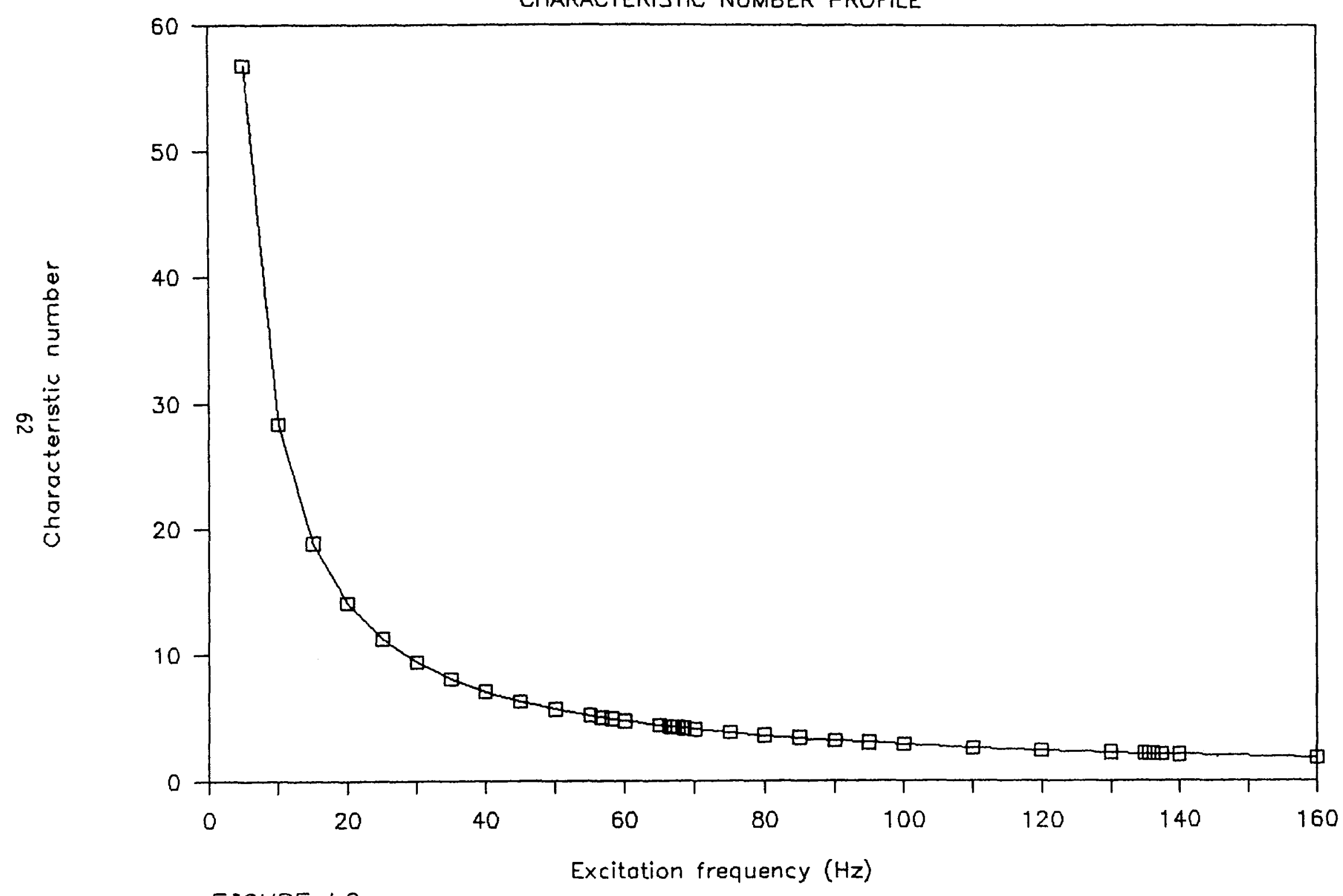

FIGURE 4.2 
TRANSMISSION LINE: IBERALL'S ANALYSIS

INS. CAVITY/EXCITATION PRES. AMP. RATIO

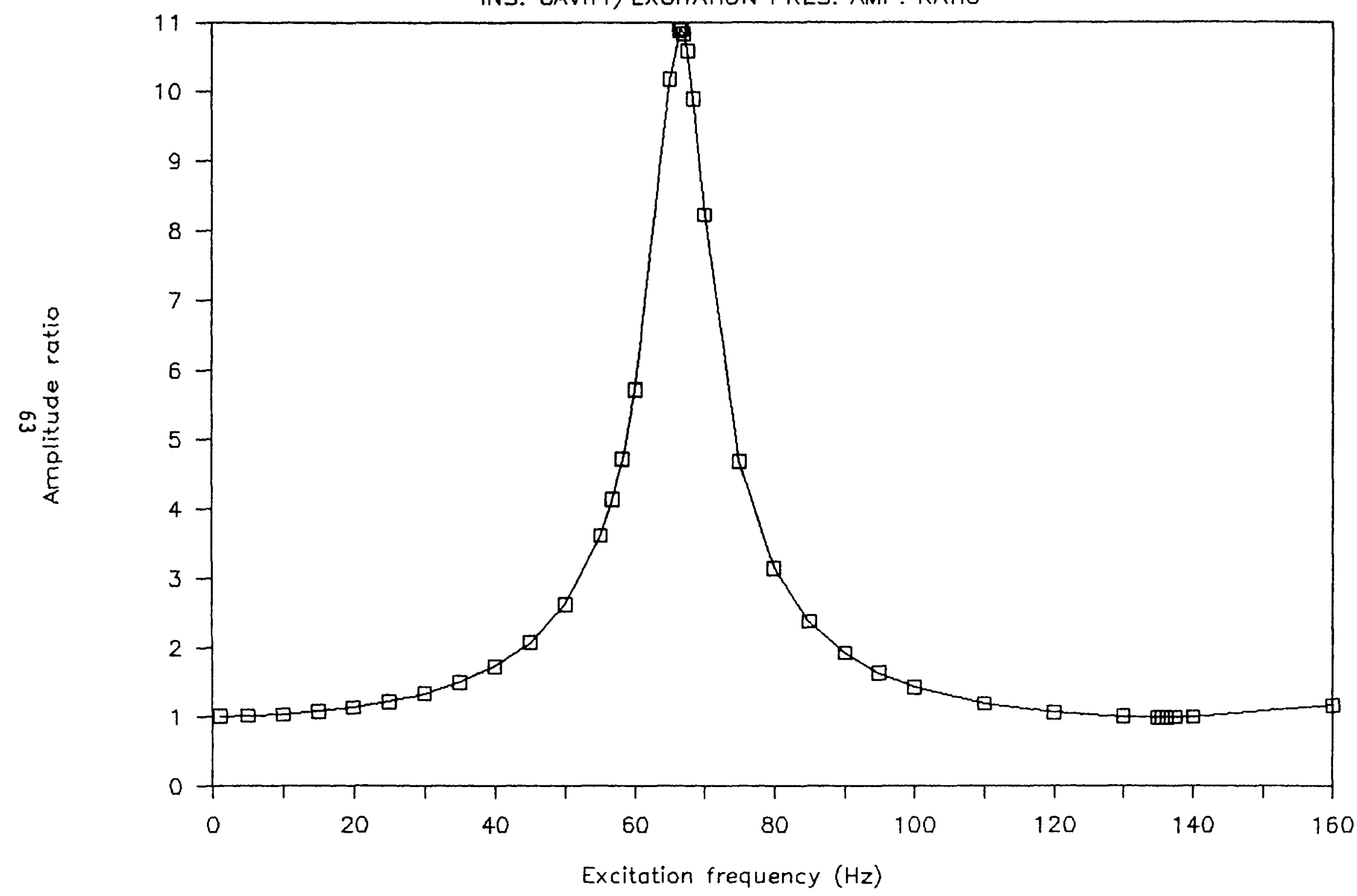

FIGURE 4.3 


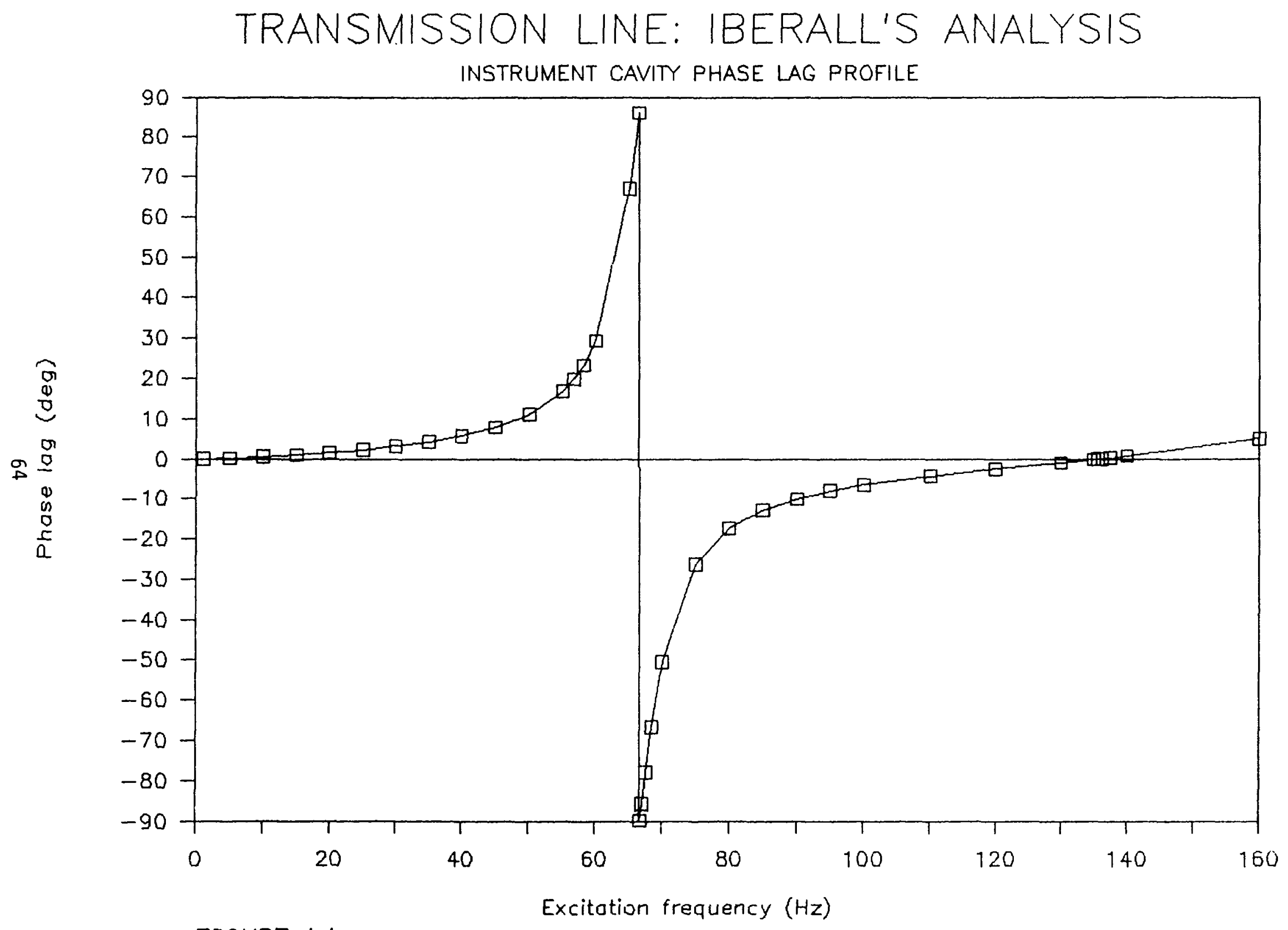

FIGURE 4.4 
the first resonant peak. The topologies of figure 4.3 and 4.4 are typical of acoustic phenomena occurring in tubes. Of particular note is that the first resonance occurs at an excitation frequency of approximately $66.6 \mathrm{~Hz}$, which corresponds to a characteristic number of about 4.3 . This may be compared against a characteristic number of 2 (Ch64) predicted by acoustic theory. The difference in characteristic numbers shows the impact of the irreversibilities and non-linearities included in Iberall's analysis.

Using figures 4.2 and 4.3 as a guide, the simulation has been exercised at analytic characteristic numbers of $5.6,10.0$, and 24.1 . These values correspond, respectively, to conditions close to resonance, midway between resonance and quiescence, and far from resonance. The comparison between the simulation and analysis at a characteristic number of 5.6 is summarized by figures 4.5 and 4.6. In these figures, the amplitude ratio and phase lag are plotted as a function of the number of integration increments per cycle. In this context, the lower bound of the variation range corresponds to the number of integration increments produced by the UPD algorithm (that is, the first or leftmost point plotted is generated by the UPD algorithm) while all the other points are generated using the equilibrium algorithm. The demarcated profiles are generated by the simulation while the unmarked horizontal lines depict the analytical values.

Figure 4.5 clearly shows that the simulated amplitude ratio asymptotes toward about 2.3 with increasing temporal discretisation (or number of increments per cycle). At 200 increments/cycle the discrepancy between the simulated and analytical amplitude ratio amounts to 148 . However, the UPD algorithm ( 6 increments/cycle) produces an amplitude ratio discrepancy of almost 548. An examination of figure 4.6 shows a similar trend for the phase angle behavior in which the simulated values asymptote toward about $5.8 \mathrm{deg}$, yielding a discrepancy of 48.48 with the 11.251 deg phase 1 ag produced by Iberall's analysis. The UPD algorithm produces phase lags of 38 and 34 deg at the instrument cavity pressure profile minimum and maximum, respectively, indicating a mean discrepancy of 2208 .

These comparison data generated under conditions close to resonance indicate that two mechanisms are operating simultaneously, one numerical and one physical. Numerically, at low characteristic numbers (below 20), the numerical accuracy of the UPD algorithm is insufficient to represent adequately the transient phenomena taking place. In other words, there is a certain minimum temporal discretisation below which use of the UPD algorithm is not warranted as a result of its numerical accuracy limitations. Physically, information propagation effects similar to those described by Jimenez (Ji73) are occurring. These effects result in a progressive steepening of the pressure wave until such time as at the onset of resonance, a discontinuity, or shock, forms. Because both the equilibrium and UPD information propagation algorithms do not describe shock formation phenomena, the algorithms are invalid in the presence of pressure wave discontinuities (Go87). Hence, should resonance-induced pressure wave discontinuities exist in the SPDE, for example, then neither the UPD nor the equilibrium algorithm could, by definition, correctly model the information propagation. However, in view of the SPDE having $N_{c h}>20$, the existence of such discontinuities is considered unlikely. 


\section{TRANSMISSION LINE: SIMULATION}

CHARACTERISTIC NO. $=5.6$

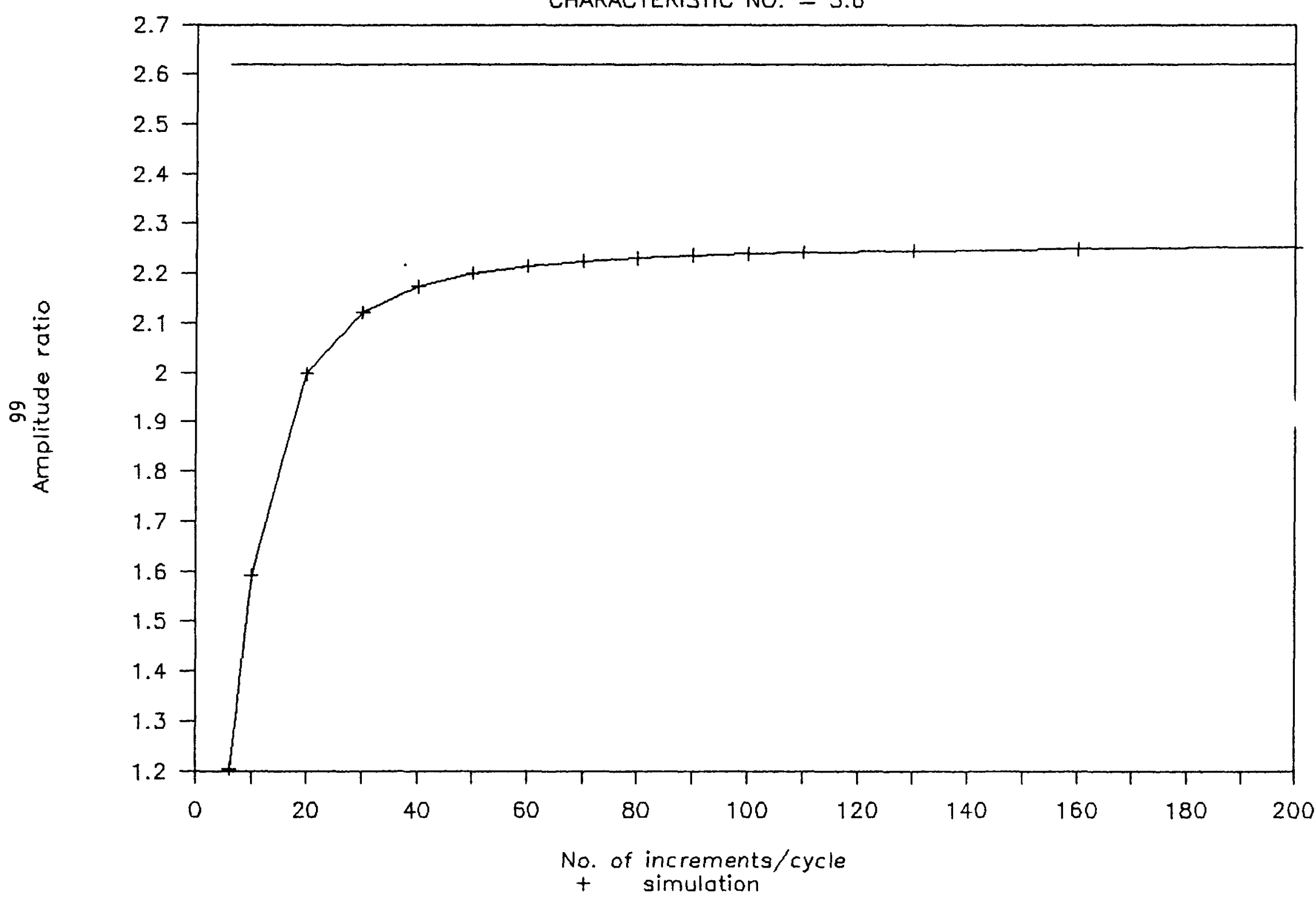

FIGURE 4.5 
TRANSMISSION LINE: SIMULATION

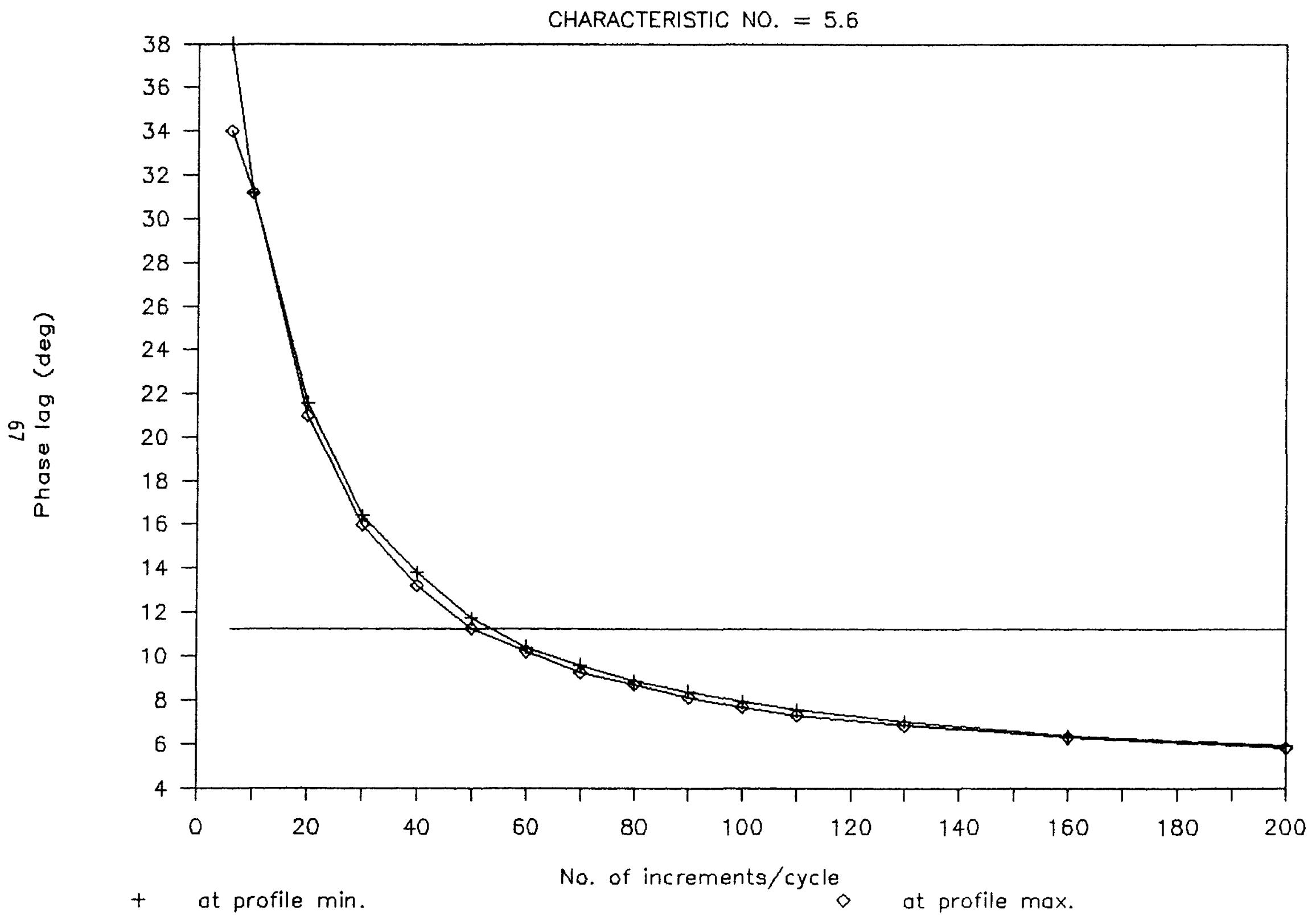

FIGURE 4.6 
Figures 4.7 and 4.8 depict the amplitude ratio and phase lag behavior at a characteristic number of 10 (excitation amplitude and frequency of .022 bars and $23.8 \mathrm{~Hz}$ respectively). The profiles show the same topologies as those of figures 4.5 and 4.6 except that the phase lags at the instrument cavity pressure profile maximum and minimum appear to be more divergent because of the plot scaling. The asymptotic phase lag discrepancy is reduced to about 338 from the 488 noted under conditions closer to resonance.

Similarly, the asymptotic amplitude ratio discrepancy is reduced to 48 . Hence the observations made above are applicable under these conditions as well with the additional note that the influence of information propagation effects becomes less pronounced the further the operating point from resonance.

Lastly, at a characteristic number of 24.1 (excitation amplitude and frequency of .05 bars and $11.7 \mathrm{~Hz}$, respectively), figures 4.9 and 4.10 show that conditions are sufficiently far from resonance that only numerical effects remain. In figure 4.9 , the amplitude ratio discrepancy varies between 1.18 and .98 for the UPD and equilibrium algorithms, respectively. Figure 4.10 effectively shows that the simulated phase lags bound the analytic phase lag in a somewhat random fashion which, owing to their smallness, is symptomatic of numerical effects. As the SPDE also yields a characteristic number of 24, these data show that the temporal accuracy of the UPD algorithm is just adequate to portray the information propagation behavior of the SPDE with some qualitative confidence although, quantitatively, the representational accuracy of the results is probably somewhat less than that suggested by the small SPDE simulation energy balance errors achieved.

\subsubsection{Pseudo-SPDE Geometry}

The invariant parameters used to define the pseudo-SPDE geometry are listed in table 4.2 .

Table 4.2 Pseudo-SPDE parameters.

\begin{tabular}{lc}
\hline Transmission line length (mm) & 321.25 \\
Transmission line internal diameter(mm) & 1.397 \\
Instrument cavity diameter (mm) & 14 \\
Instrument cavity volume (cm^3) & .414 \\
Working fluid & Helium \\
Mean excitation pressure (bar) & 150.29 \\
Mean system temperature (deg C) & 100
\end{tabular}




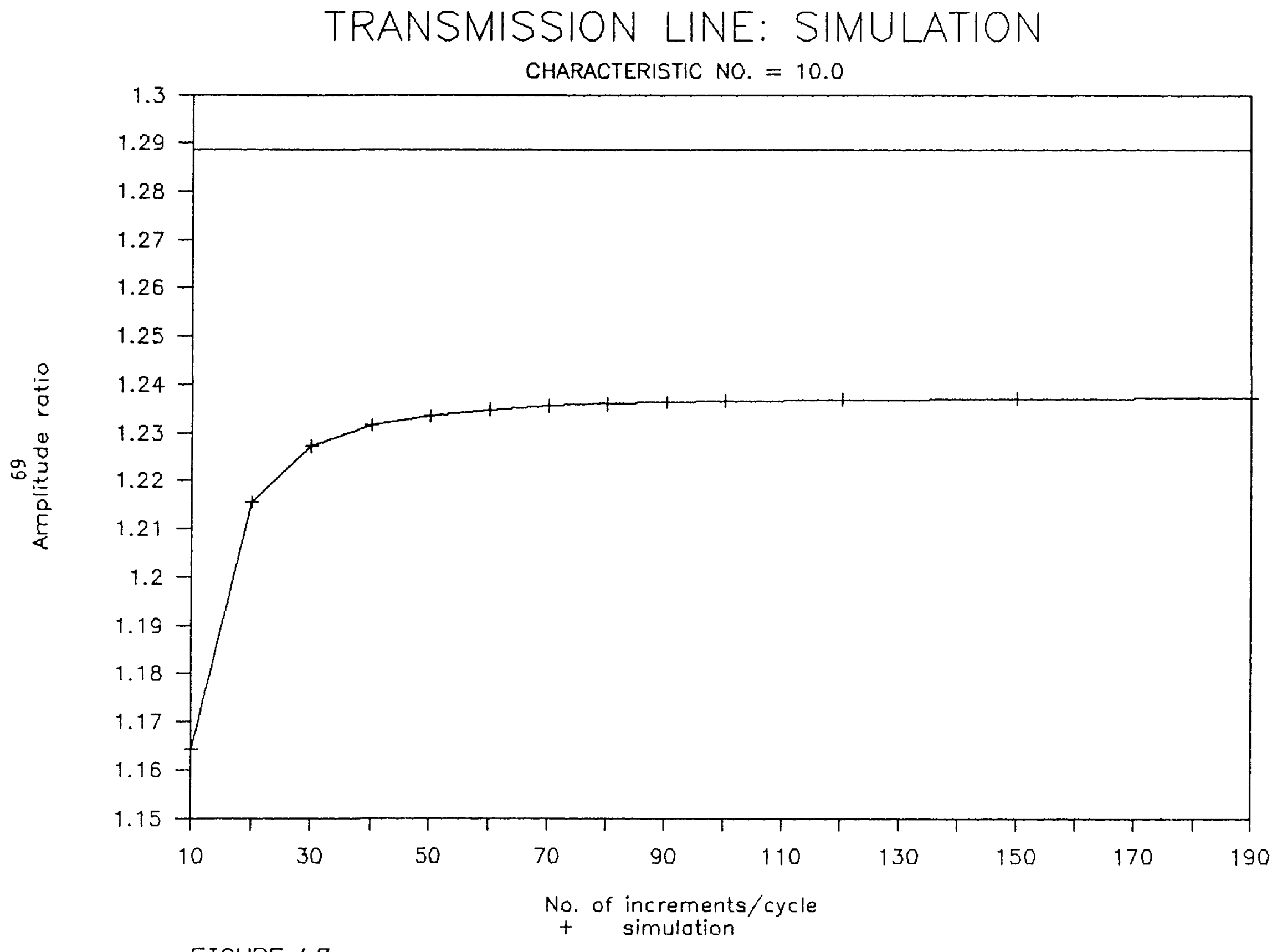

FIGURE 4.7 


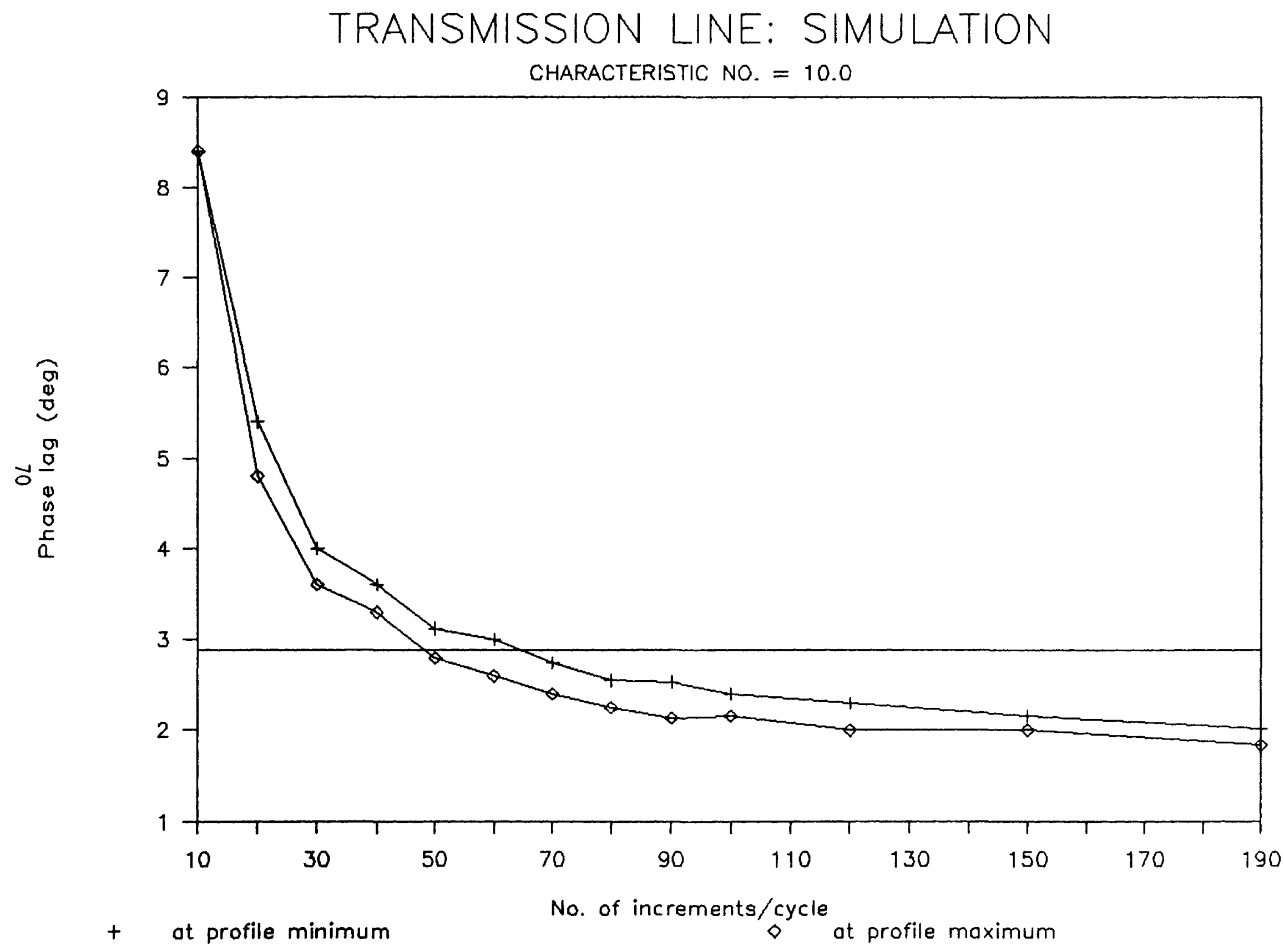

FIGURE 4.8 
TRANSMISSION LINE: SIMULATION

CHARACTERISTIC NO. $=24.1$

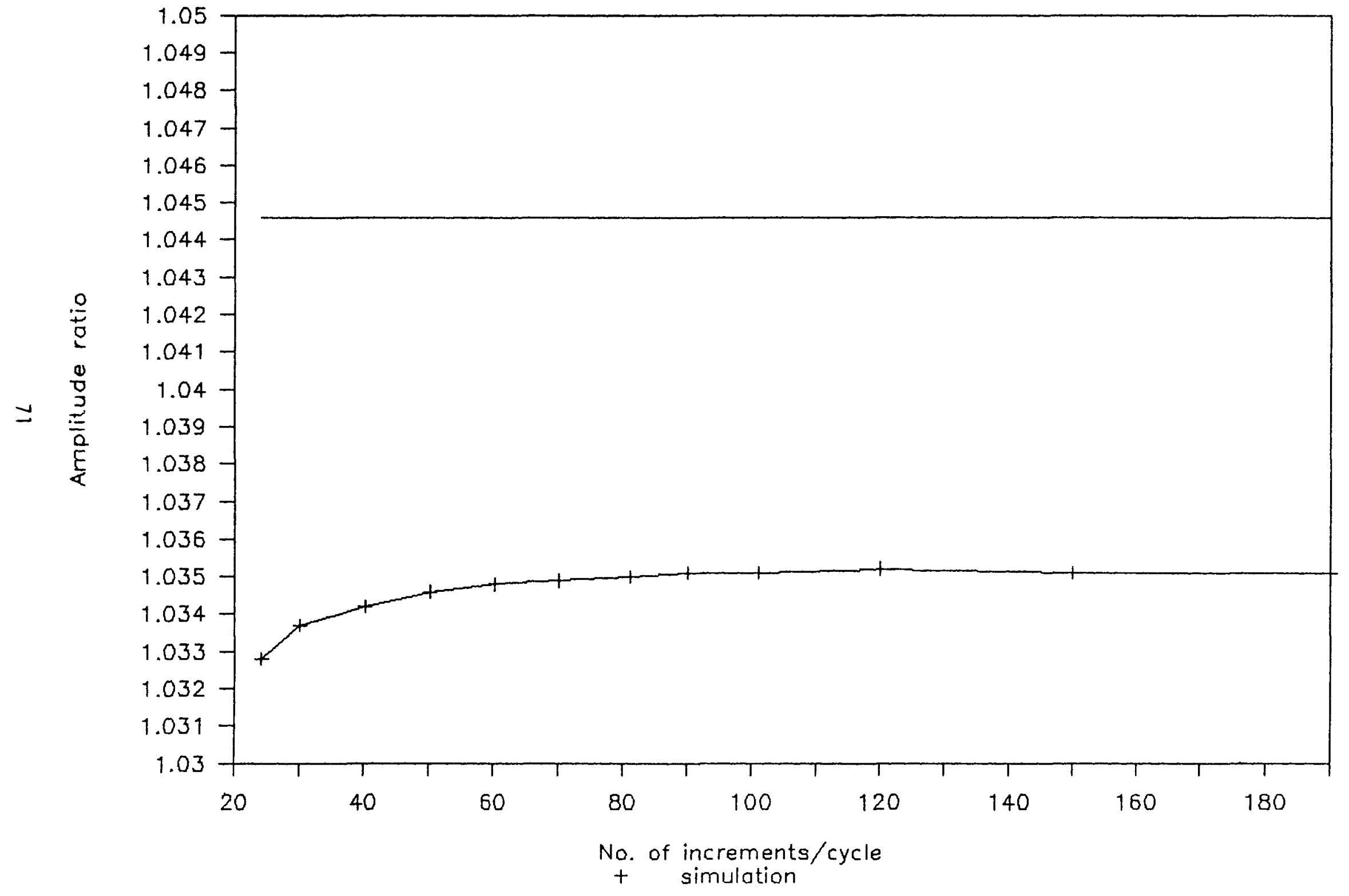

FIGURE 4.9 


\section{TRANSMISSION LINE: SIMULATION}

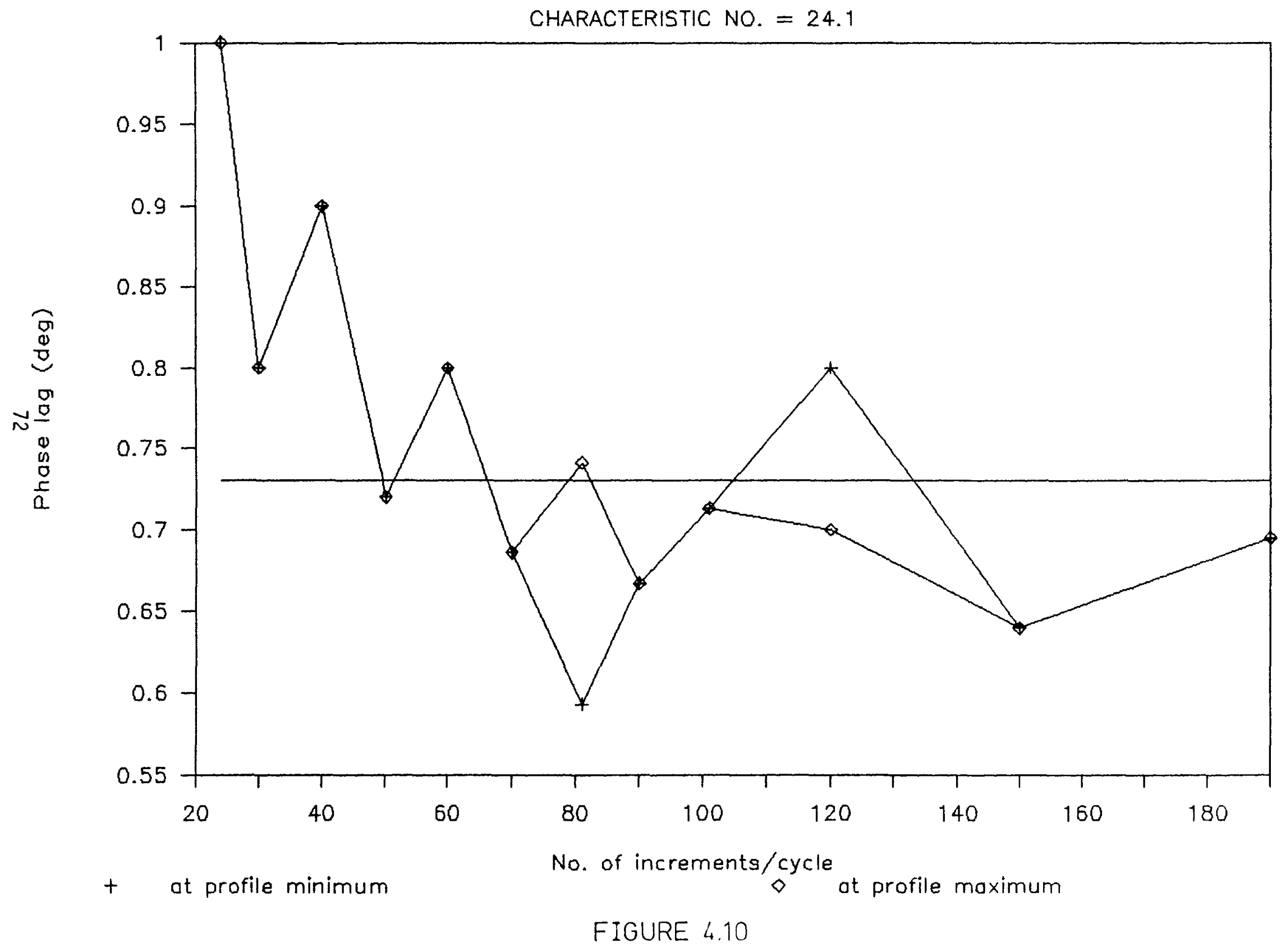


In selecting this geometrical configuration, no attempt has been made to take account of the effect of either the expansion or the compression space by increasing the volume of the instrument cavity. Although Iberall and Goldschmied report data for volume ratios of as much as 50 to 1 , in the case of the SPDE expansion space for example, using the tube data of table 2, the cavity-to-tube volume ratio amounts to about 522 to 1 . In terms of the validation data reported by Watts and Goldschmied as well as the assumptions made in Iberall's analysis (see section 4.3), there does not appear to be any solid basis for extrapolating the analysis by an order of magnitude in volume ratio terms. Furthermore, the mechanism of pressure wave generation in the SPDE is very different from that used by Iberall. Hence the main value of Iberall's analysis in terms of understanding the gas dynamics of the SPDE is to test whether closed tube resonance effects are a likely cause of the observed phenomena. In this context, it thus seems desirable to maintain the congruency of the tube boundary conditions with those that produced the high level of agreement between Watts' experimental data and Iberall's analysis.

Using an excitation amplitude of .9 bars, the results of applying Iberall's analysis to the pseudo-SPDE geometry are shown in figures 4.11 to 4.13. Figures 4.11 and 4.12 show that the first resonant peak occurs at an excitation frequency of $504.8 \mathrm{~Hz}, 5.1$ times larger than the nominal $100 \mathrm{~Hz}$ operating frequency. At $100 \mathrm{~Hz}$, the amplitude ratio amounts to 1.05 while the phase lag is .327 deg (figure 4.13) and the characteristic number is 35.2 . Hence even though the pseudo-SPDE geometry characteristic number is greater than that of the actual SPDE (approximately 24), these results show that closed tube resonant effects are not likely to be the cause of the observed SPDE phase angle behavior (the difference in characteristic numbers serves to increase the conservative nature of this inference).

A comparison of the simulated and analytical data for the pseudo-SPDE geometry is given in figures 4.14 and 4.15. The comparison is performed at a frequency of $99.569 \mathrm{~Hz}$ (taken from an MTI test) and an excitation amplitude of .9 bars. Figure 4.14 yields an amplitude ratio discrepancy of .558 for the UPD algorithm, increasing to .658 for the equilibrium algorithm at 90 increments per cycle. It is interesting to note that in this case the simulation over-predicts the amplitude ratio while in the previous case (in which the transmission line is 3.8 times longer) the simulation under-predicts the amplitude ratio. This is a probable result of the neglect of the advection terms in the momentum equation which Iberall uses to produce equation (116) ( Ib50).

[In the second paragraph of section 4 on page 100 of Ib50, Iberall makes reference to the fact that his analysis is based on Kirchoff's equations of sound. Tracing this to the relevant equation quoted by Rayleigh (St26, page 315 , equation 13), in the class of problems being considered, Iberal1 apparently agrees with Rayleigh's assertion (St26, page 3) that "...Whenever the motion is very small, the (advective) terms $u d u / d x$, etc., diminish in relative importance, and ultimately, $\mathrm{D} / \mathrm{Dt}=\mathrm{d} / \mathrm{dt}$ (or the substantive and total derivatives are equal)." This assumption does not apply to SPDE conditions nor does it lend credence to cases in which large instrument cavities produce fluxes big enough to produce significant momentum transport. These 


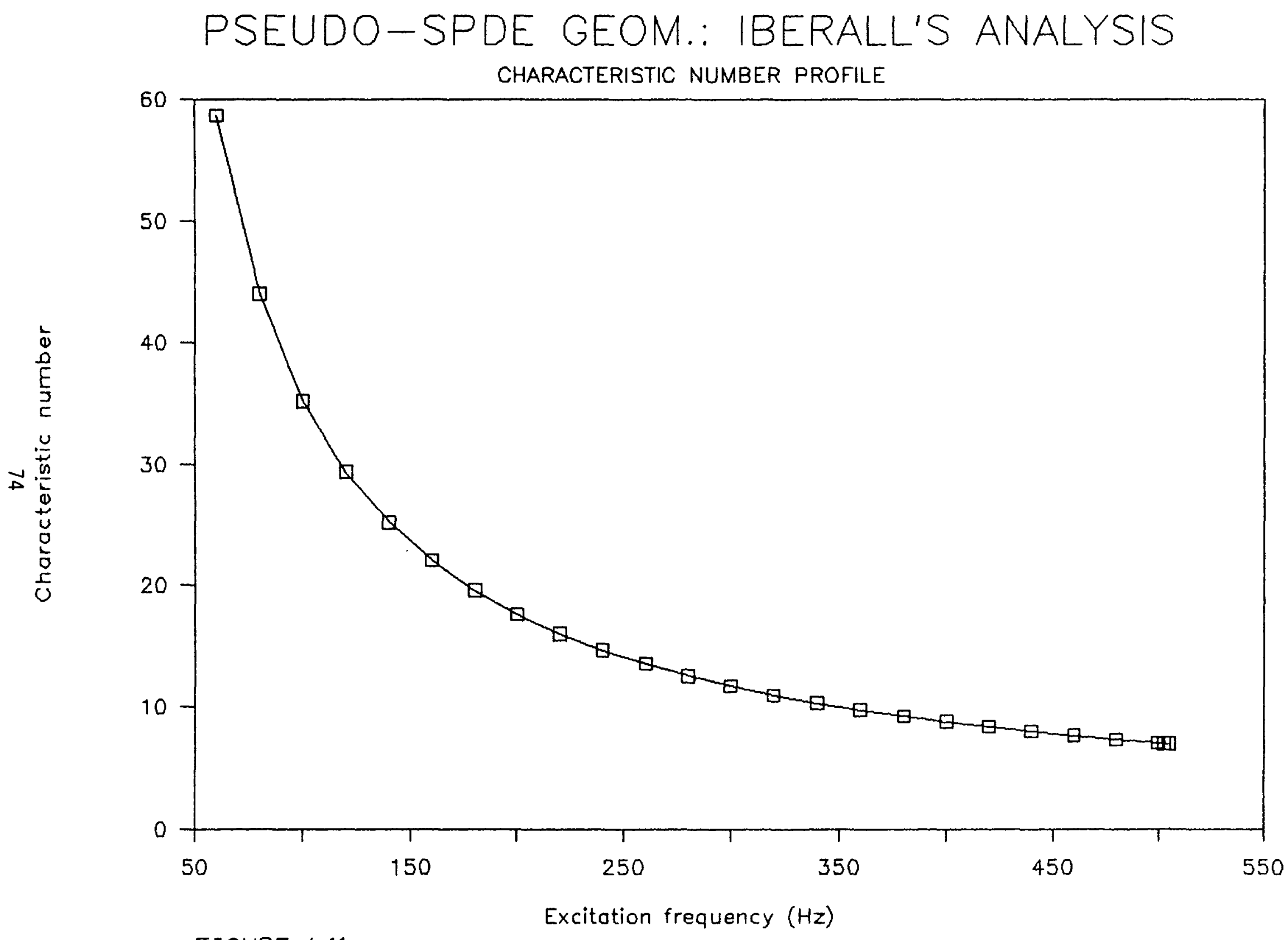

FIGURE 4.11 


\section{PSEUDO-SPDE GEOM.: IBERALL'S ANALYSIS}

INS. CAVITY/EXCITATION PRES. AMP. RATIO

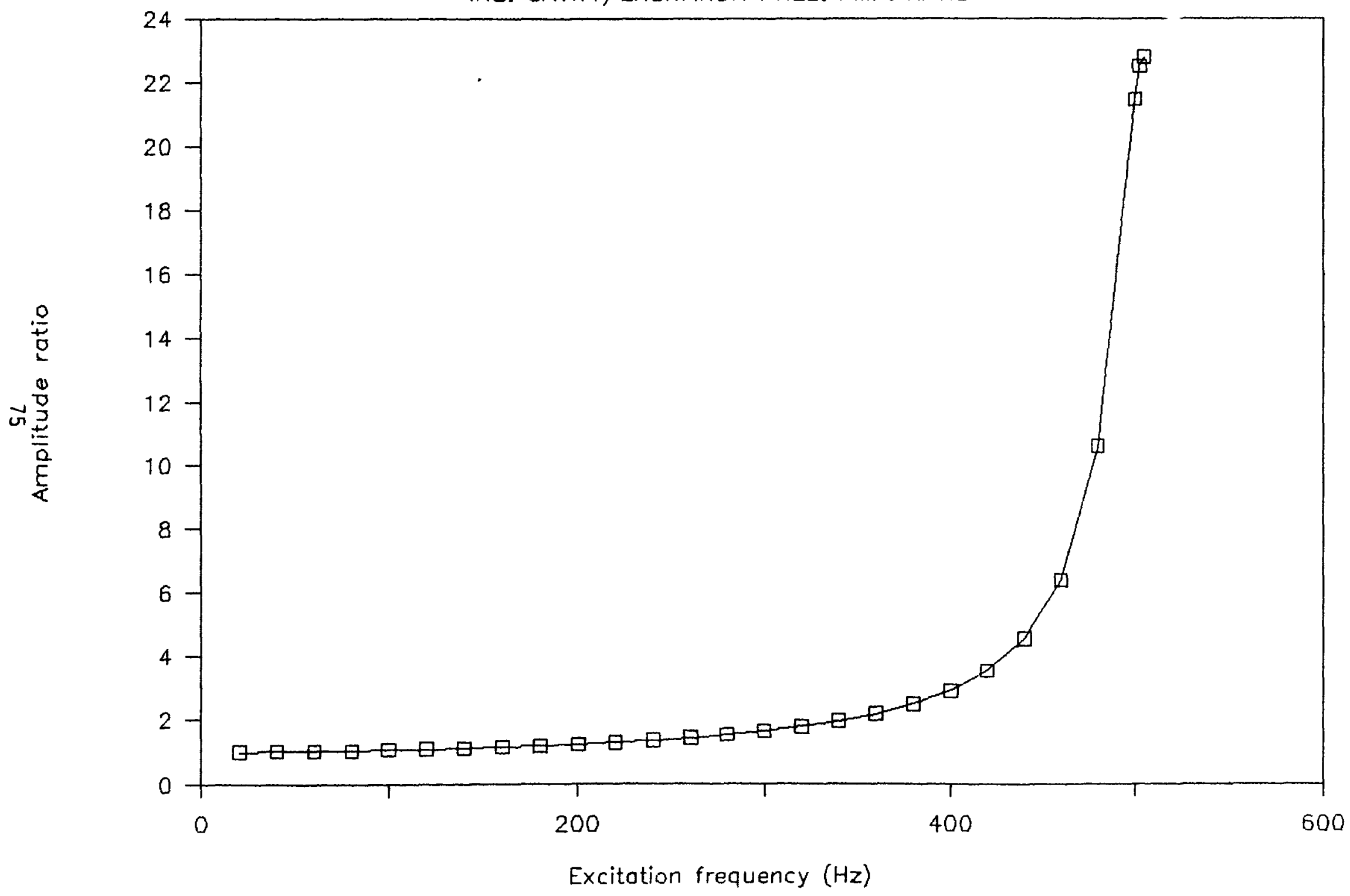

FIGURE 4.12 


\section{PSEUDO-SPDE GEOM.: IBERALL'S ANALYSIS}

INSTRUMENT CAVITY PHASE LAG PROFILE

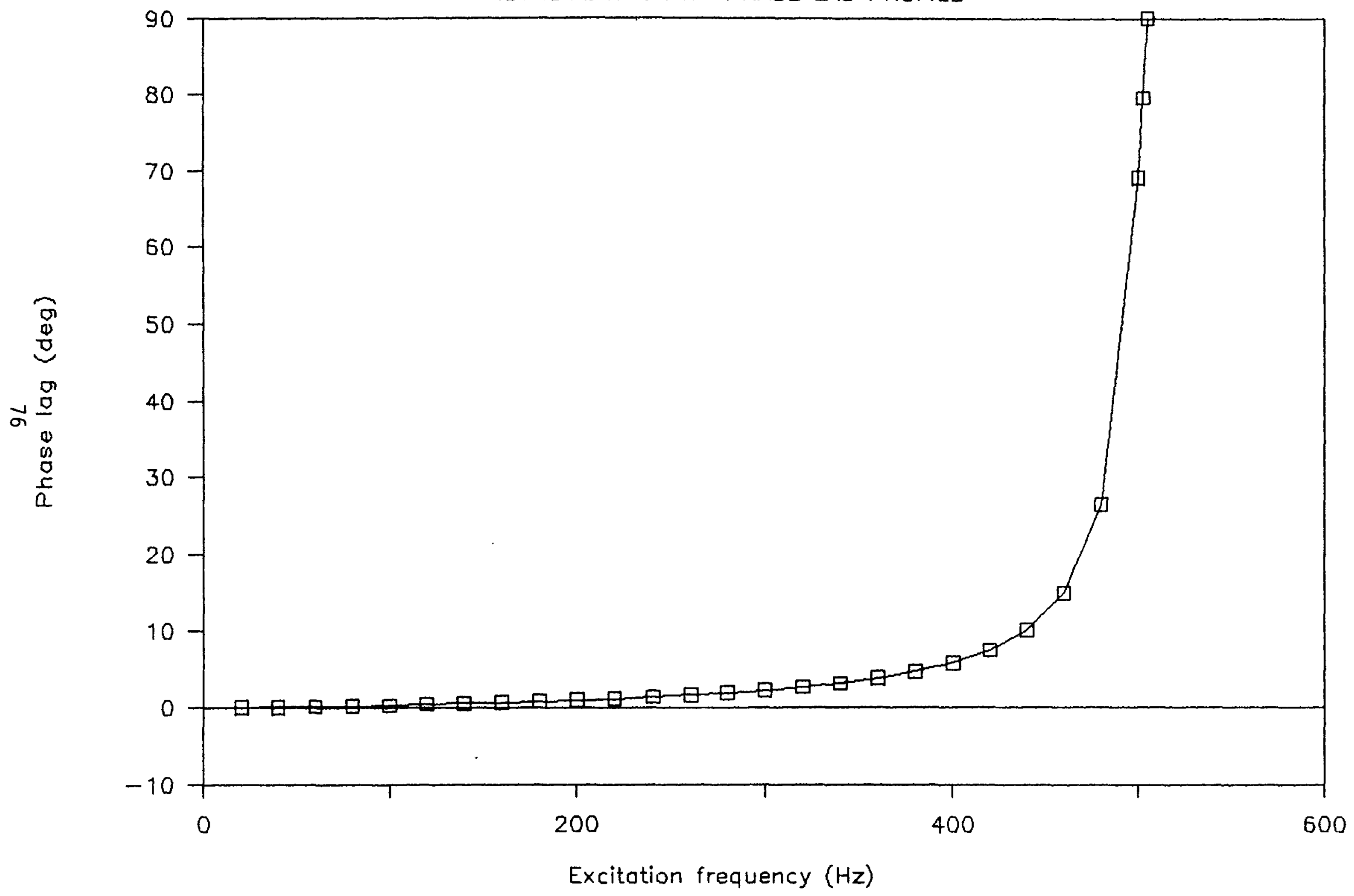

FIGURE 4.13 


\section{PSEUDO-SPDE GEOMETRY: SIMULATION \\ CHARACTERISTIC NO. $=35.3$}

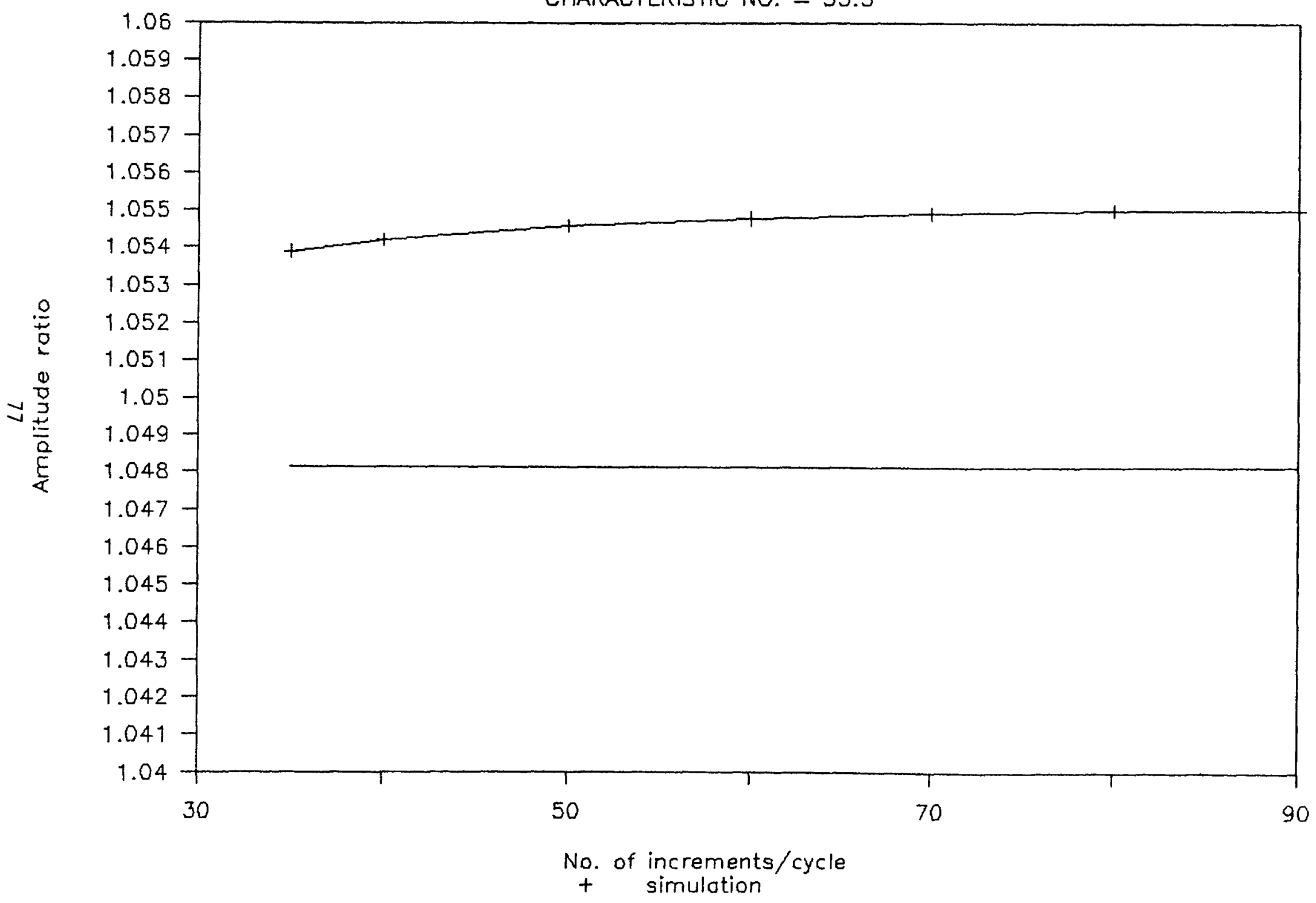

F IGURE 4.14

No. of increments/cycle 


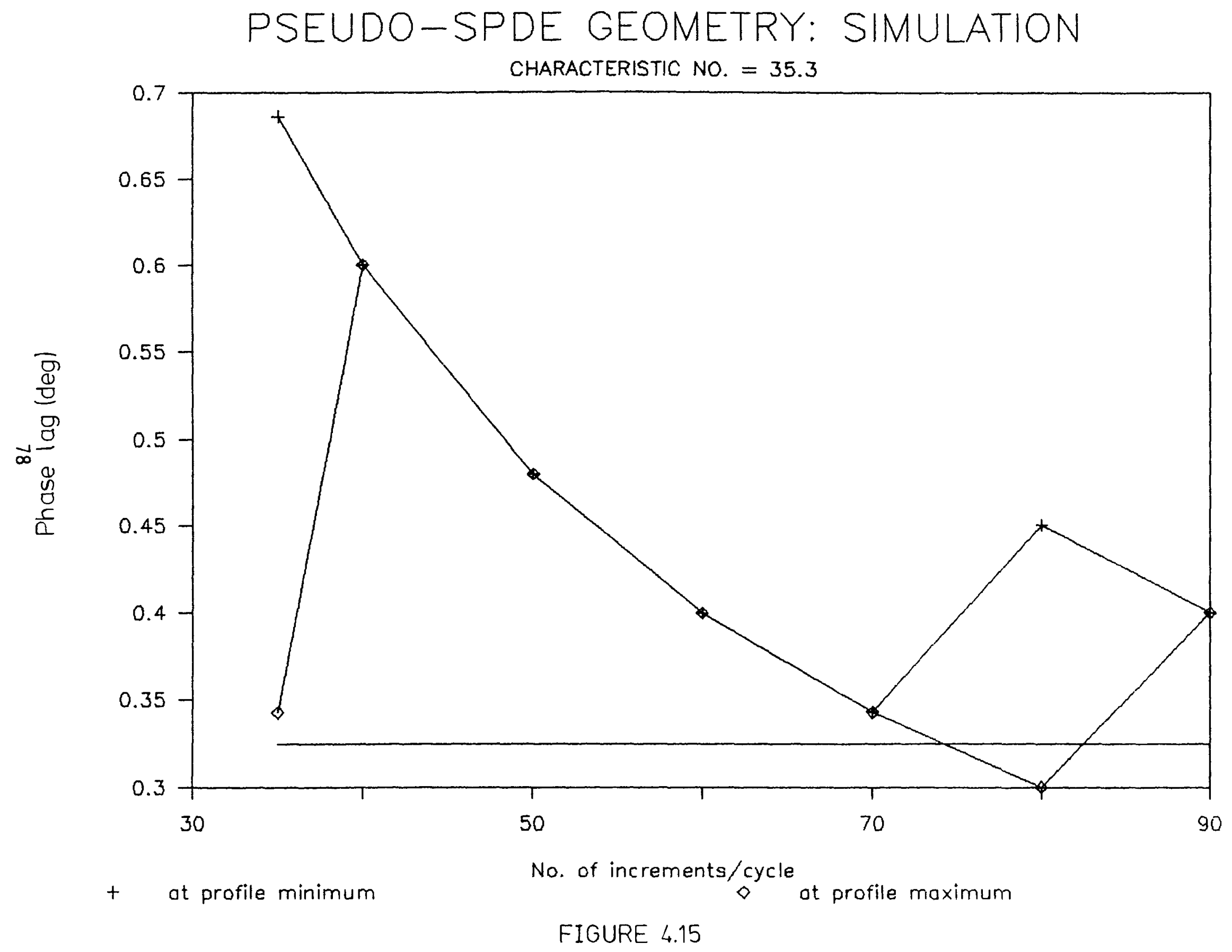


limitations should be borne in mind when comparing the simulated results (which include all the advective terms) with their analytic counterparts.]

Due to the smallness of the phase lags, figure 4.15 is inconclusive in revealing any trends that cannot be ascribed to systematic numerical effects, in particular those produced by the cubic spline fitting procedure.

\subsection{INTERPRETATION}

Under resonant conditions in which information propagation effects are responsible for the observed transmission line behavior, the inadequacy of the equilibrium information propagation hypothesis is demonstrated. This is manifested by the equilibrium algorithm producing results that asymptote towards values which are not in agreement with those produced by Iberall's analysis. Hence, should such effects exist in the SPDE, they would not be properly accounted for by an equilibrium information propagation hypothesis.

As both the UPD and equilibrium algorithms are based on a temporal integration scheme which is only first order accurate, the comparison data show that the algorithms quickly lose their physical validity at temporal resolutions below 20 to 30 increments per cycle. Hence, use of the UPD algorithm to simulate the SPDE at an effective temporal resolution of 23 to 24 increments/cycle is somewhat risky as the integration algorithm is operating in a region of marginal temporal accuracy. Therefore, the comparison exercise is inconclusive in determining whether the UPD algorithm accurately can portray the information propagation behavior of the SPDE in a quantitative sense.

In other words, the data show that the numerical errors produced by a first order accurate, implicit integration algorithm at time steps large enough to satisfy the UPD hypothesis at low characteristic numbers do not permit an assessment of the physical accuracy of the UPD algorithm to be made. This conclusion in effect reflects the limitations of the pressure-linking algorithm itself, which is defined in essence by equation (2.47). Since the off-diagonal pressure term coefficients are given as a product of the time step $\Delta t$ and a geometrical factor $A^{2} / V$, these coefficients are positive definite and are independent of the working fluid state. Therefore, an implicit solution of the pressure equation (2.47) implies infinite information propagation irrespective of the time step. Thus, satisfying the UPD hypothesis is necessary physically in order to ensure that the actual and numerical information propagation rates match. Decreasing the time step towards the equilibrium limit thus effectively increases the information propagation rate towards infinity. Therefore the data show that the numerical effect of the time step size predominates, submerging the simultaneously occurring physical effect.

Thus before any further assessment of the physical relevance of the information propagation hypothesis is attempted, the impact of numerical accuracy on the UPD algorithm in particular needs investigation. 


\subsection{NUMERICAL ACCURACY CONSIDERATIONS}

The physical adequacy of the first order temporal accuracy of the simulation code at low characteristic numbers may be evaluated by using a more accurate temporal integration scheme. The issue of significance in this endeavour is to find a temporally more accurate scheme which does not destroy the hypothesized information propagation modelling characteristics of the UPD algorithm in particular. After studying the Gedeon GLIMPS methodology (Ge86) and further review of the literature, the following observations can be made:

a.

The GLIMPS code is a particular case of a general class of numerical algorithms which may be conveniently referred to as 'multistep implicit' methods. In these methods, a given time increment is resolved into $m$ sub-steps so that each sub-step is solved implicitly via a single matrix inversion, with both $m$ and the magnitude of the time increment being arbitrary. Each sub-step $m$ may be further divided into $n$ increments so that the order of the temporal gradient approximation used in each sub-step is restricted only by the requirement that $k \leq n$, where $k$ is the number of increments required to implement a particular integration scheme. For example, a 5th order backward difference approximation would require $n \geq k=6$.

Hence the GLIMPS code uses a time increment equal to the cycle period with one sub-step (that is, $m=1$ ) with $n$ apparently being in the range of 6 to 10 and $k=3$. In contrast, the 'UPD simulation' has $n=k=1$ with $m$ set equal to the integer nearest the characteristic number. Hence, if the numerical accuracy of multi-step implicit methods is a monotonically increasing function of $n$, then a generalised version of the GLIMPS' code (without the cyclic equilibrium closure restriction, in particular) theoretically should be physically valid for characteristic numbers $\geq 1$. However, as a general proposition, multi-step implicit methods should be valid for all characteristic numbers, although it is hard to conceive of Stirling machines being built with characteristic numbers approaching 1 .

b. It is possible to construct a fully implicit temporal integration procedure based on the Crank-Nicholson scheme, which is second order accurate, while retaining the information propagation characteristics of the UPD algorithm.

Another approach to testing the numerical accuracy of the algorithms is to implement the full pressure domain splitting (PDS) algorithm discussed in section 2.6. However, in view of the large increment in computational effort associated with the PDS algorithm and, therefore, NASA's reluctance to pursue this approach, it was decided to limit the numerical accuracy investigation of the 'UPD simulation' to an implementation of the Crank-Nicholson integration scheme.

\subsection{THE CRANK-NICHOLSON INTEGRATION SCHEME}

A generalised two-step temporal integration scheme may be defined by: 
If $K=0$, equation (4.1) represents an explicit scheme of $0(\Delta t)$; if $K=$ 1 , equation (4.1) is an implicit scheme of $O(\Delta t)$; and, if $K=.5$ then equation (4.1) represents a Crank-Nicholson scheme of $O\left(\Delta t^{2}\right)$.

Thus, choosing $K=.5$ and discretising:

$\psi_{(t+\Delta t)} / \Delta t-.5 \mathrm{~d} \psi / \mathrm{d} t_{(t+\Delta t)} \approx \psi_{(t)} / \Delta t+.5 \mathrm{~d} \psi / \mathrm{d} t(t)$

Hence by setting $d \psi / d t(t)$ of the current time step equal to $d \psi / d t(t+\Delta t)$ of the previous time step (which by definition is implicitly determined), the Crank-Nicholson scheme retains much of the stability of the fully implicit scheme as confirmed by von Neumann stability analysis (Ro82), although the method is not unconditionally stable. Stability problems arise when $\Delta t$ becomes large enough for some Fourier modes to overshoot, in which case, the $O\left(\Delta t^{2}\right)$ error terms for the Crank-Nicholson scheme will exceed the $O(\Delta t)$ error terms for the implicit scheme. Furthermore, equation (4.2) retains the information propagation characteristics of the first order implicit scheme used in the UPD simulation.

\subsection{RESULTS}

The parameters describing the transmission line used as the basis for the comparison exercise are listed in table 4.3. These parameters correspond to those given in Go68 table $\mathrm{V}$ and Wa65 figure 6 .

Table 4.3 Transmission line parameters.

\begin{tabular}{lc}
\hline Transmission line length (mm) & 1220 \\
Transmission line internal diameter (mm) & 4.66 \\
Instrument cavity length (mm) & 1 \\
Instrument cavity volume (cm^3) & .414 \\
Gas & Air \\
Mean excitation pressure (bar) & .77 \\
Excitation pressure amplitude (bar) & .00077 \\
Mean system temperature (deg C) & 28.98
\end{tabular}

The computerised implementation of Iberall's analysis has been carefully rechecked against Watts' results given in Wa65 table 6 to ensure exact agreement.

The simulation parameters used are given in table 4.4 
Table 4.4 Simulation parameters.

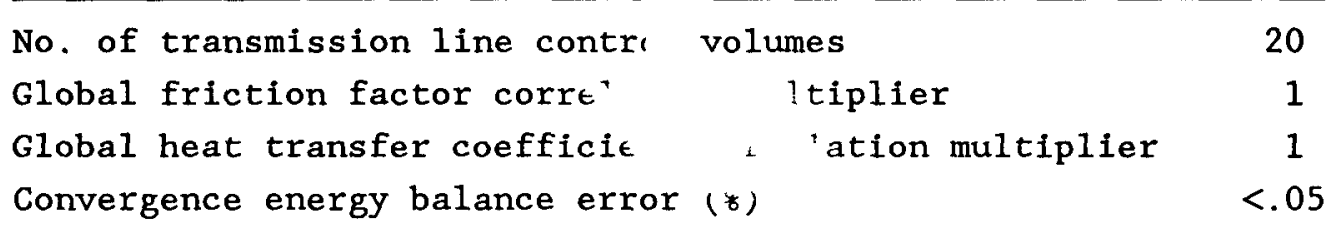

The results of exercising the UPD algorithm using both the implicit and Crank-Nicholson integration schemes against Iberall's analysis are listed in table 4.5 as a function of frequency (and, therefore, of $C:$ aracteristic number since $N_{c h}$ is inversely proportional to frequency). The tabulated data are plotted in figures 4.16 to 4.19 .

In all cases, the amplitude ratio errors produced by the CrankNicholson scheme (CN-s) are less than those produced by the implicit scheme (I-S). The CN-s phase angle errors are also less than their I-s counterparts for $N_{c h}>8.15$. Below this, the time steps are large enough so that the $\mathrm{CN}-\mathrm{s}$ truncation errors exceed those of the $I-s$ as noted in section 4.8 above.

It is also evident that the phase angle differences between the $\mathrm{CN}-\mathrm{s}$ and I-s are larger than the amplitude ratio differences, which is consistent with the notion that information propagation effects are more apparent, numerically, in the phase angles.

If one accepts that a 108 discrepancy represents a reasonable level of validation (see Go87, section 7.7.4), then based on the errors reported in table 4.5, the CN-s is valid for $N_{c h} \geq 13.27$ while the I-s is valid for $N_{c h} \geq$ 20.38 . The accuracy differences are also far more pronounced for the phase angles than for the amplitude ratios as shown by figures 4.17 and 4.19.

Based on this discussion, the UPD and equilibrium algorithms are compared at $N_{c h}$ of $8.15,13.27$, and 20.38 , respectively corresponding to cases in which neither the $\mathrm{CN}-\mathrm{s}$ nor the I-s schemes are valid, only the $\mathrm{CN}-\mathrm{s}$ is valid, and both schemes are valid. The results are given in tables 4.6 and plotted in figures 4.20 through 4.31 .

In all cases, amplitude ratio errors decrease with decreasing integration time step (increasing number of increments per cycle). However, the amplitude ratios asymptote to a constant value beyond 50 increments per cycle. With the exception of figures 4.29 and 4.31 , the phase angle errors also reveal this asymptotic behavior except that the limiting error is about 508 for $N_{c h}<20$. The behavior of the $\mathrm{CN}-\mathrm{s}$ in figures 4.29 and 4.31 may be inconsistent and requires further study before comment. 


\section{UNITARY PRESSURE DOMAIN ALGORITHM Amplitude Ratio Comparison}

$\begin{array}{ccc}\text { lberall } & \text { Crank-Nicholson } & \text { Implicit } \\ \text { * } & -+- & --\not X--\end{array}$

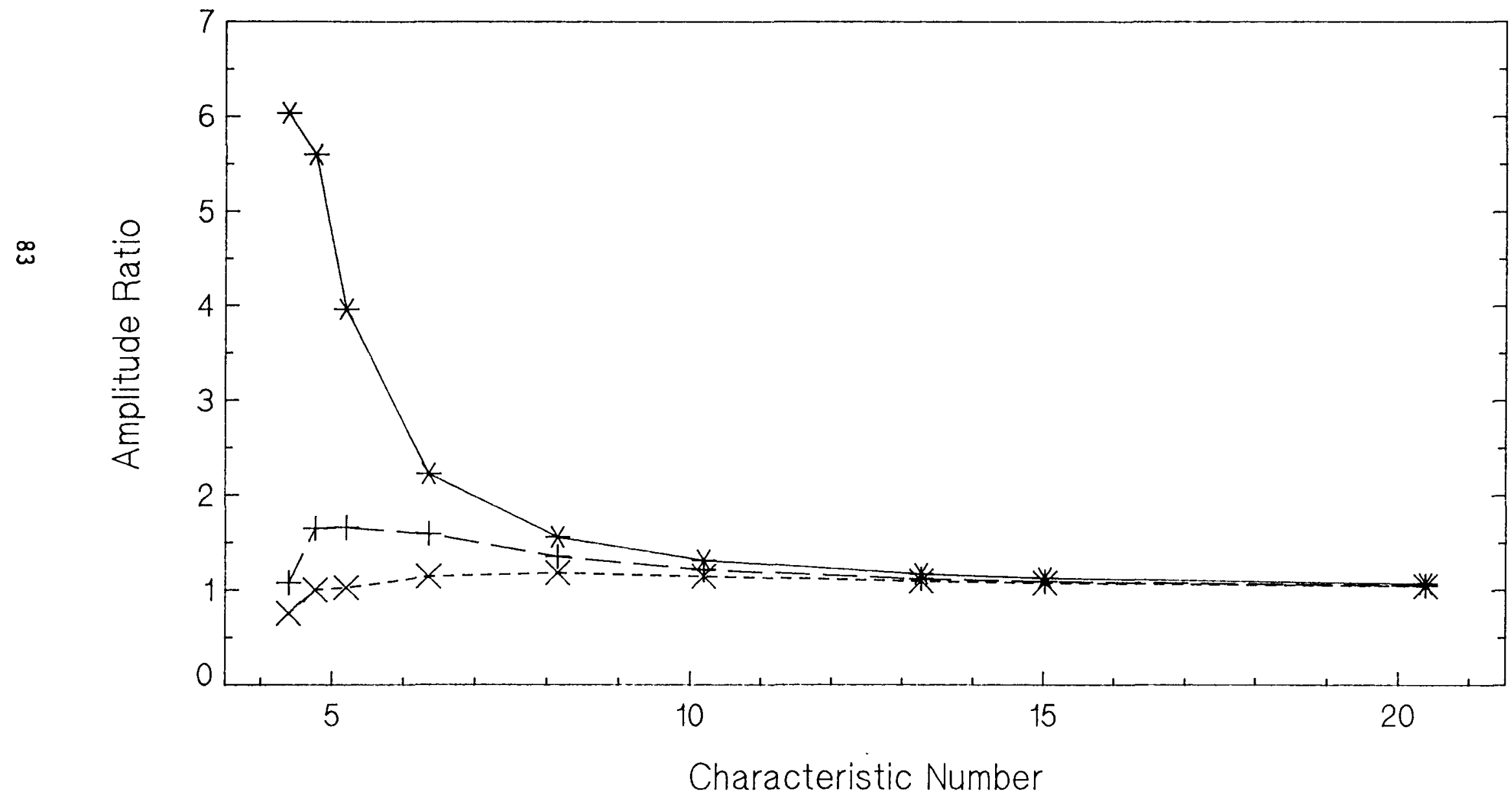

FIGURE 4.16 


\section{UNITARY PRESSURE DOMAIN ALGORITHM Amplitude Ratio Errors}

Crank-Nicholson

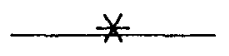

Implicit

$-\neq$

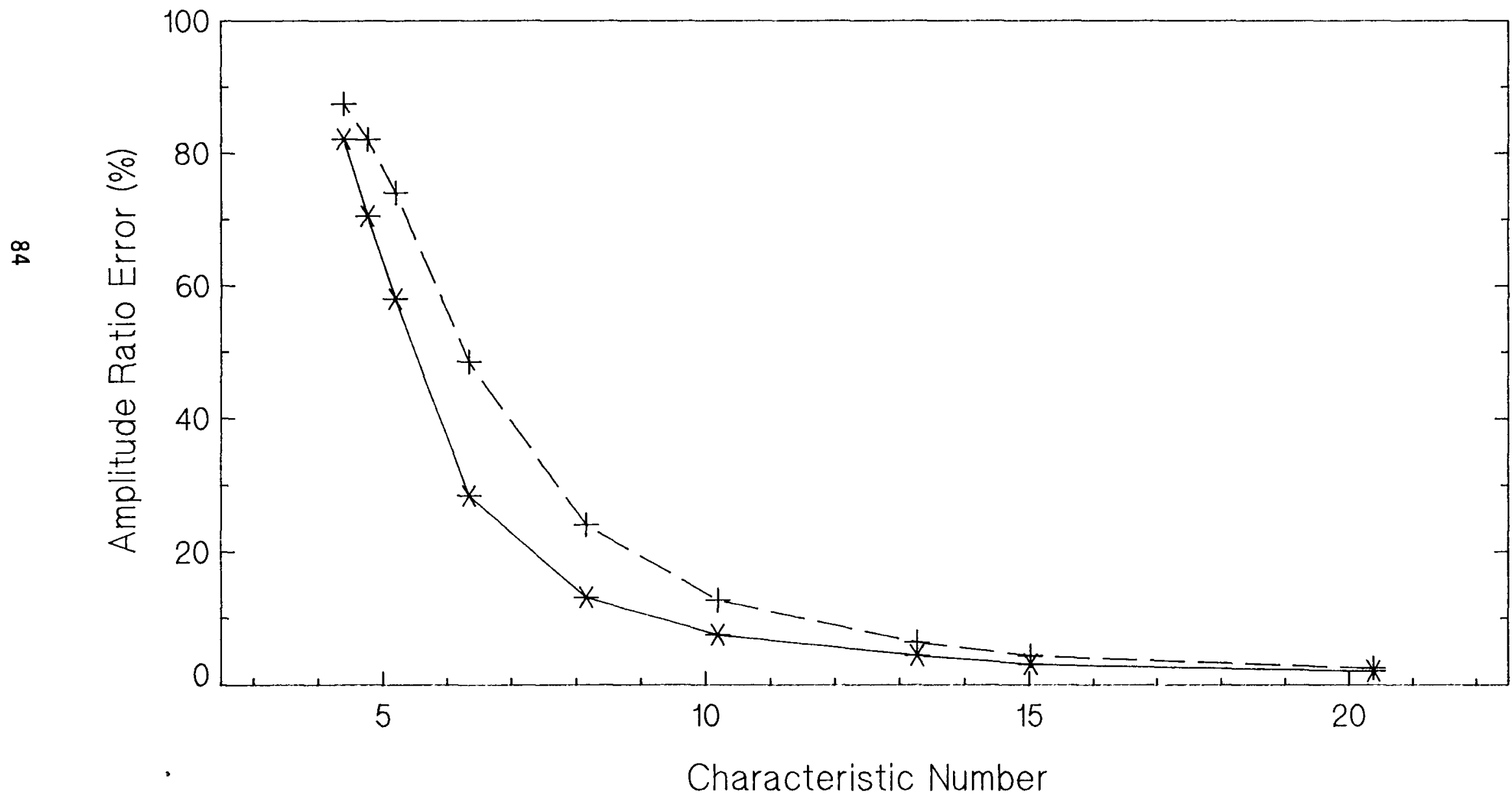

FIGURE 4.17 


\section{UNITARY PRESSURE DOMAIN ALGORITHM}

Phase Angle Comparison

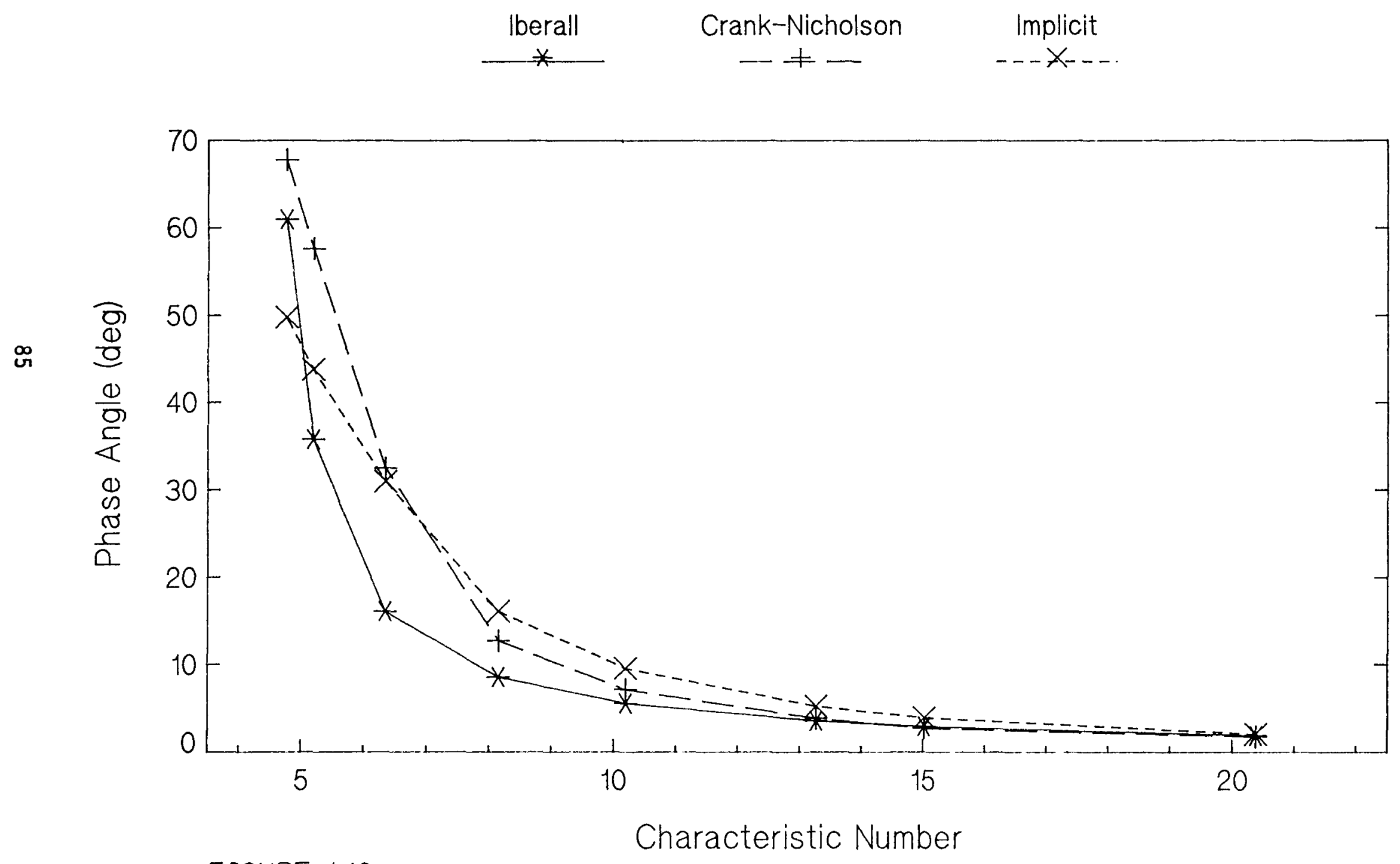

FIGURE 4.18 


\section{UNITARY PRESSURE DOMAIN ALGORITHM Phase Angle Errors}

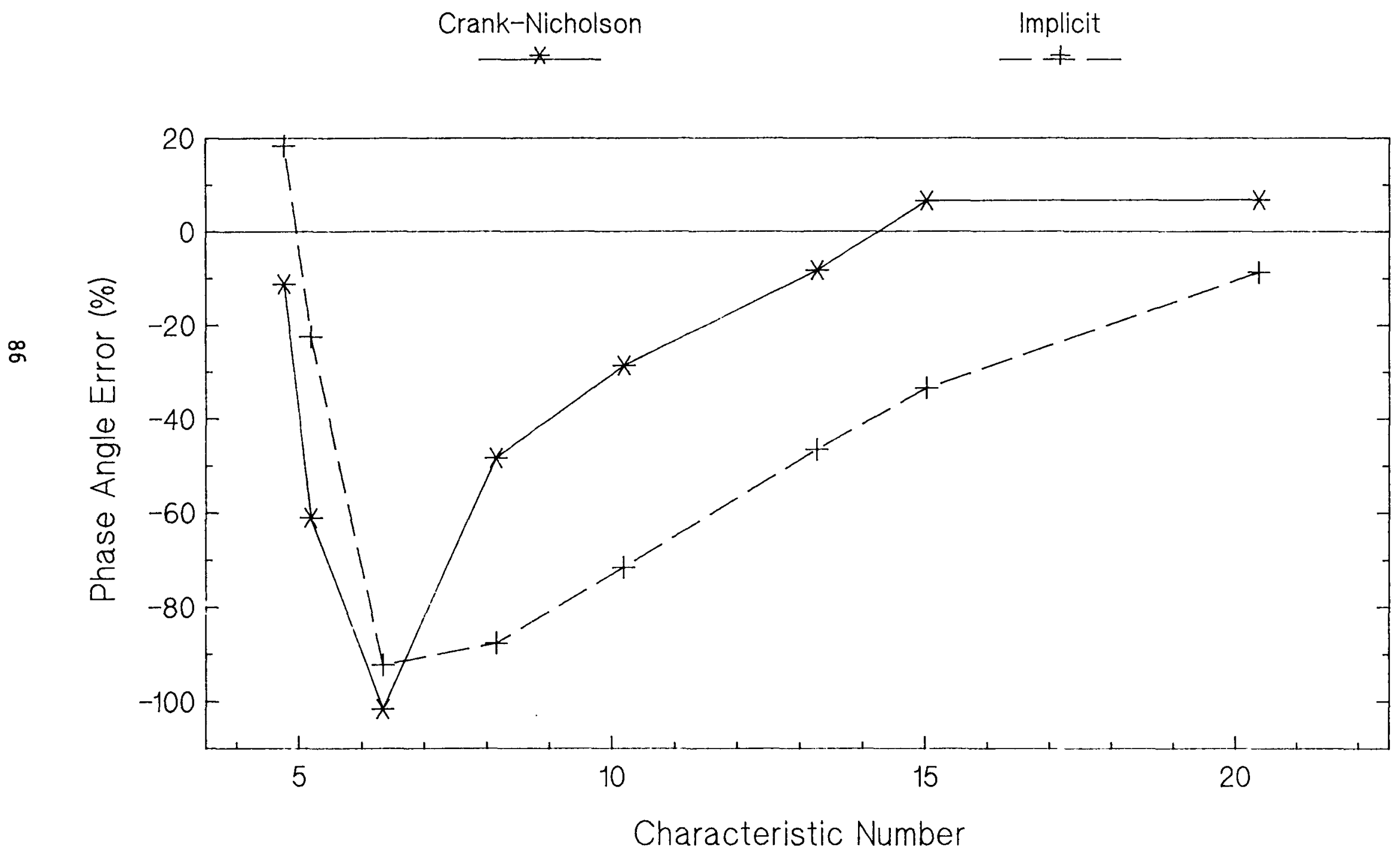

FIGURE 4.19 
Table 4.5 UPD algorithm: Comparison of Crank-Nicholson and implicit integration routines. Data: Watts figure 6; Goldschmeid table $V$

\begin{tabular}{|c|c|c|c|c|c|c|}
\hline \multirow[t]{2}{*}{$\begin{array}{l}\text { Frequency } \\
\text { (Hz) }\end{array}$} & \multirow[t]{2}{*}{$\begin{array}{c}\text { Characteristic } \\
\text { No. }\end{array}$} & \multicolumn{3}{|c|}{$\begin{array}{c}\text { Ampl itude } \\
\text { Ratio }\end{array}$} & \multicolumn{2}{|c|}{ Error } \\
\hline & & Iberall & Crank-Nic. & Implicit & Crank-Nic. & Implicit \\
\hline $\begin{array}{c}14 \\
19 \\
21.5 \\
28 \\
35 \\
45 \\
55 \\
60 \\
65\end{array}$ & $\begin{array}{l}20.38 \\
15.02 \\
13.27 \\
10.19 \\
8.15 \\
6.34 \\
5.19 \\
4.76 \\
4.39\end{array}$ & $\begin{array}{c}1.0719 \\
1.13 \\
1.175 \\
1.318 \\
1.56 \\
2.228 \\
3.9611 \\
5.5996 \\
6.035\end{array}$ & $\begin{array}{c}1.0495 \\
1.0941 \\
1.1219 \\
1.2175 \\
1.3548 \\
1.5943 \\
1.6619 \\
1.65 \\
1.0776\end{array}$ & $\begin{array}{l}1.0446 \\
1.0799 \\
1.0987 \\
1.1497 \\
1.1844 \\
1.1454 \\
1.0288 \\
1.0038 \\
0.7562\end{array}$ & $\begin{array}{c}2.09 \\
3.18 \\
4.52 \\
7.63 \\
13.15 \\
28.44 \\
58.04 \\
70.53 \\
82.14 \\
\end{array}$ & $\begin{array}{c}2.55 \\
4.43 \\
6.49 \\
12.77 \\
24.08 \\
48.59 \\
74.03 \\
82.07 \\
87.47 \\
\end{array}$ \\
\hline
\end{tabular}

\begin{tabular}{|c|c|c|c|c|c|c|}
\hline \multirow{2}{*}{$\begin{array}{l}\text { Frequency } \\
(\mathrm{Hz})\end{array}$} & \multirow{2}{*}{$\begin{array}{l}\text { Characteristic } \\
\text { No. }\end{array}$} & \multicolumn{3}{|c|}{$\begin{array}{l}\text { Phase } \\
\text { (deg) }\end{array}$} & \multicolumn{2}{|c|}{ Error } \\
\hline & & Iberall & Crank-Nic. & Implicit & $\frac{(\zeta)}{\text { Crank-Nic. }}$ & Imolicit \\
\hline 14 & 20.38 & 1.932 & 1.8 & 2.1 & 6.83 & -8.70 \\
\hline 19 & 15.02 & 2.999 & 2.8 & 4.0 & 6.64 & -33.38 \\
\hline 21.5 & 13.27 & 3.624 & 3.923 & 5.308 & -8.25 & -46.47 \\
\hline 28 & 10.19 & 5.6 & 7.2 & 9.6 & -28.57 & -71.43 \\
\hline 35 & 8.15 & 8.6 & 12.75 & 16.13 & -48.26 & -87.56 \\
\hline 45 & 6.34 & 16.129 & 32.5 & 31 & -101.50 & -92.20 \\
\hline 55 & 5.19 & 35.783 & 57.6 & 43.8 & -60.97 & -22.40 \\
\hline 60 & 4.76 & 60.983 & 67.8 & 49.8 & -11.18 & 18.34 \\
\hline 65 & 4.39 & -78.605 & 83.25 & 48 & & \\
\hline
\end{tabular}


Table 4.6 Equilibrium algorithm: Comparison of Crank-Nicholson and implicit integration routines.

Table 4.6.1 Frequency $=35 \mathrm{~Hz}$ Characteristic no. $=8.15$

\begin{tabular}{|c|c|c|c|c|c|c|c|c|}
\hline \multirow{2}{*}{$\begin{array}{l}\text { Increments } \\
\text { per Cycle }\end{array}$} & \multicolumn{4}{|c|}{ Amplitude Ratio } & \multicolumn{4}{|c|}{ Phase Angle } \\
\hline & \multicolumn{2}{|c|}{ Magnitude } & \multicolumn{2}{|c|}{ Error (\%) } & \multicolumn{2}{|c|}{ Magni tude (deg) } & \multicolumn{2}{|c|}{ Error (\%) } \\
\hline $\begin{array}{c}8 \\
16 \\
32 \\
64 \\
128 \\
256 \\
\end{array}$ & $\begin{array}{c}\text { Crank-Nic. } \\
1.3548 \\
1.3916 \\
1.4028 \\
1.4071 \\
1.4091 \\
1.41\end{array}$ & $\begin{array}{c}\text { Implicit } \\
1.1844 \\
1.3294 \\
1.3824 \\
1.4001 \\
1.4064 \\
1.4088\end{array}$ & $\begin{array}{c}\text { Crank-NiC. } \\
13.15 \\
10.79 \\
10.08 \\
9.80 \\
9.67 \\
9.62\end{array}$ & $\begin{array}{c}\text { Implicit } \\
24.08 \\
14.78 \\
11.38 \\
10.25 \\
9.85 \\
9.69\end{array}$ & $\begin{array}{c}\text { Crank-Nic. } \\
12.75 \\
8.25 \\
6.375 \\
5.297 \\
4.852 \\
4.571\end{array}$ & $\begin{array}{c}\text { Implicit } \\
16.13 \\
12 \\
8.344 \\
6.375 \\
5.32 \\
4.969\end{array}$ & $\begin{array}{c}\text { Crank-Nic. } \\
-48.26 \\
4.07 \\
25.87 \\
38.41 \\
43.58 \\
46.85\end{array}$ & $\begin{array}{c}\text { Implicit } \\
-87.56 \\
-39.53 \\
2.98 \\
25.87 \\
38.14 \\
42.22\end{array}$ \\
\hline
\end{tabular}

Table 4.6.2 Frequency $=21.5 \mathrm{~Hz}$ Characteristic no. $=13.27$

\begin{tabular}{|c|c|c|c|c|c|c|c|c|}
\hline \multirow{3}{*}{$\begin{array}{l}\text { Increments } \\
\text { per Cycle }\end{array}$} & \multicolumn{4}{|c|}{ Amplitude Ratio } & \multicolumn{4}{|c|}{ Phase Angle } \\
\hline & \multicolumn{2}{|c|}{ Magni tude } & \multicolumn{2}{|c|}{ Error (\%) } & \multicolumn{2}{|c|}{ Magnitude (deg) } & \multicolumn{2}{|c|}{ Error $(\overline{\%})$} \\
\hline & Crank-NiC. & Implicit & Crank-Nic. & Implicit & Crank-NiC. & Imolicit & Crank-Nic. & Implicit \\
\hline $\begin{array}{l}13 \\
26 \\
52 \\
104 \\
208 \\
300\end{array}$ & $\begin{array}{l}1.1219 \\
1.1273 \\
1.1294 \\
1.1302 \\
1.1305 \\
1.1305\end{array}$ & $\begin{array}{l}1.0987 \\
1.1185 \\
1.1259 \\
1.1289 \\
1.1302 \\
1.1305\end{array}$ & $\begin{array}{l}4.52 \\
4.06 \\
3.88 \\
3.81 \\
3.79 \\
3.79\end{array}$ & $\begin{array}{l}6.49 \\
4.81 \\
4.18 \\
3.92 \\
3.81 \\
3.79\end{array}$ & $\begin{array}{c}3.923 \\
3.116 \\
2.538 \\
2.192 \\
2.163 \\
1.77\end{array}$ & $\begin{array}{c}5.308 \\
3.923 \\
3.115 \\
2.481 \\
2.135 \\
2.15\end{array}$ & $\begin{array}{l}-8.25 \\
14.02 \\
29.97 \\
39.51 \\
40.31 \\
51.16\end{array}$ & $\begin{array}{l}-46.47 \\
-8.25 \\
14.05 \\
31.54 \\
41.09 \\
40.67\end{array}$ \\
\hline
\end{tabular}

Table 4.6.3 Frequency $=14 \mathrm{~Hz}$ Characteristic no. $=20.38$

\begin{tabular}{|c|c|c|c|c|c|c|c|c|}
\hline \multirow{3}{*}{$\begin{array}{l}\text { Increments } \\
\text { per Cycle }\end{array}$} & \multicolumn{4}{|c|}{ Amplitude Ratio } & \multicolumn{4}{|c|}{ Phase Angle } \\
\hline & \multicolumn{2}{|c|}{ Magnitude } & \multicolumn{2}{|c|}{ Error (\%) } & \multicolumn{2}{|c|}{ Magnitude (deg) } & \multicolumn{2}{|c|}{ Error (\%) } \\
\hline & Crank-Nic. & Implicit & Crank-Nic. & Implicit & Crank-Nic. & Implicit & Crank-Nic & Implicit \\
\hline $\begin{array}{c}20 \\
35 \\
70 \\
140 \\
230 \\
\end{array}$ & $\begin{array}{l}1.0495 \\
1.0508 \\
1.0517 \\
1.0526 \\
1.0535\end{array}$ & $\begin{array}{l}1.0446 \\
1.0486 \\
1.0505 \\
1.0516 \\
1.0525\end{array}$ & $\begin{array}{l}2.09 \\
1.97 \\
1.88 \\
1.80 \\
1.72 \\
\end{array}$ & $\begin{array}{l}2.55 \\
2.17 \\
2.00 \\
1.89 \\
1.81 \\
\end{array}$ & $\begin{array}{c}1.8 \\
1.8 \\
1.672 \\
1.745 \\
1.865\end{array}$ & $\begin{array}{c}2.1 \\
1.972 \\
1.757 \\
1.715 \\
1.696 \\
\end{array}$ & $\begin{array}{c}6.83 \\
6.83 \\
13.46 \\
9.68 \\
3.47 \\
\end{array}$ & $\begin{array}{c}-8.70 \\
-2.07 \\
9.06 \\
11.23 \\
12.22 \\
\end{array}$ \\
\hline
\end{tabular}


EQUILIBRIUM ALGORITHM

Amplitude Ratio Comparison at $35 \mathrm{~Hz}$
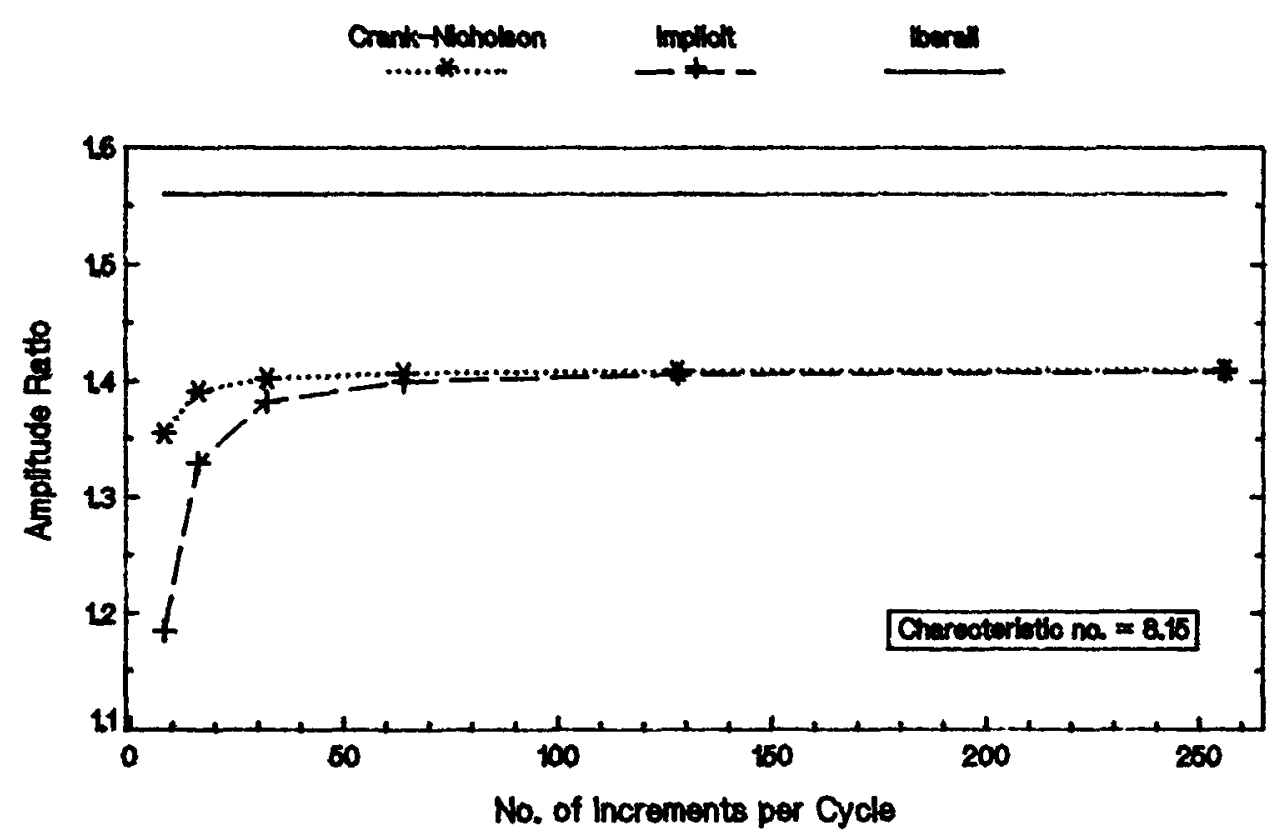

Figure 4.20

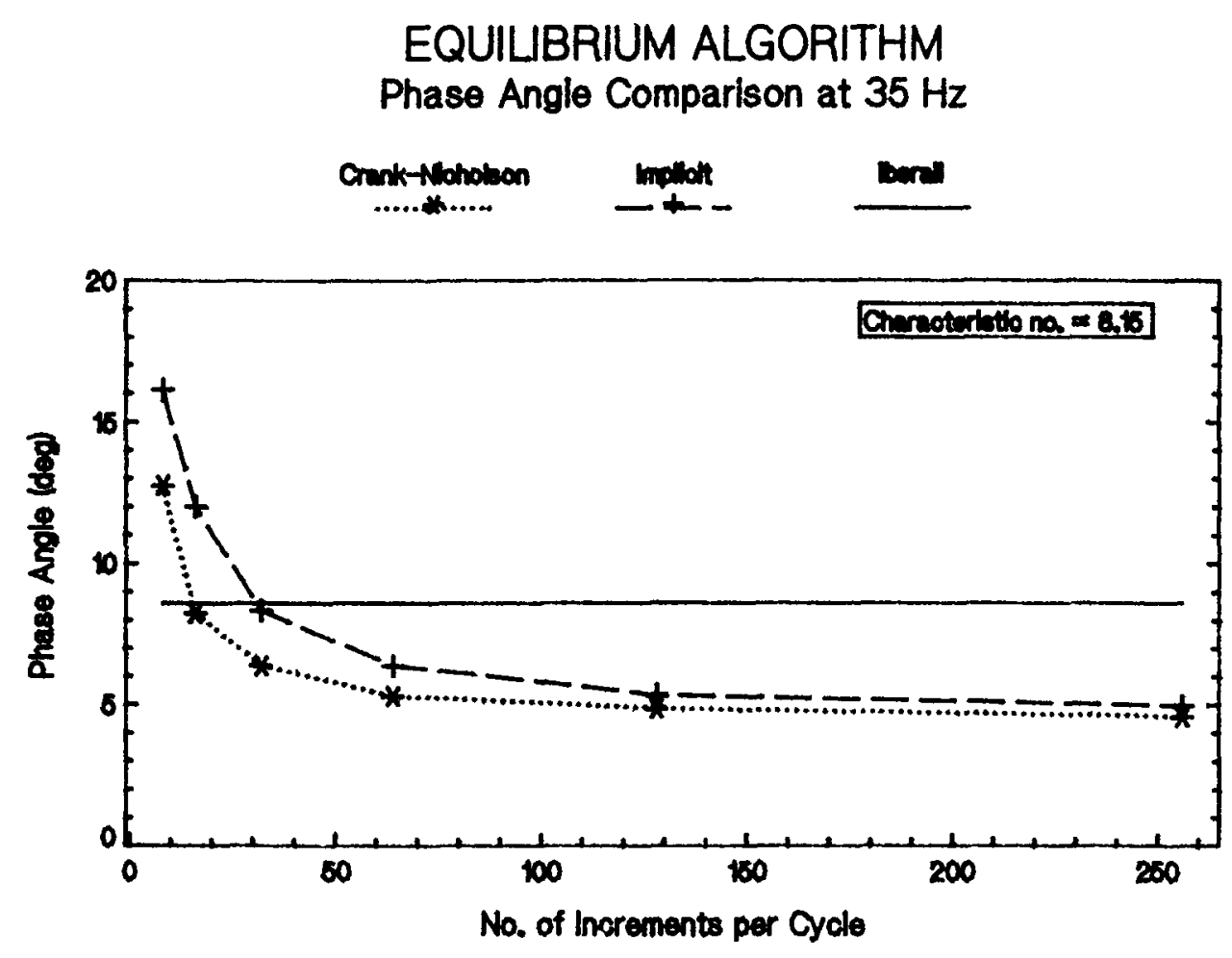

Figure 4.21 
EQUILIBRIUM ALGORITHM

Amplitude Ratio Errors at $35 \mathrm{~Hz}$

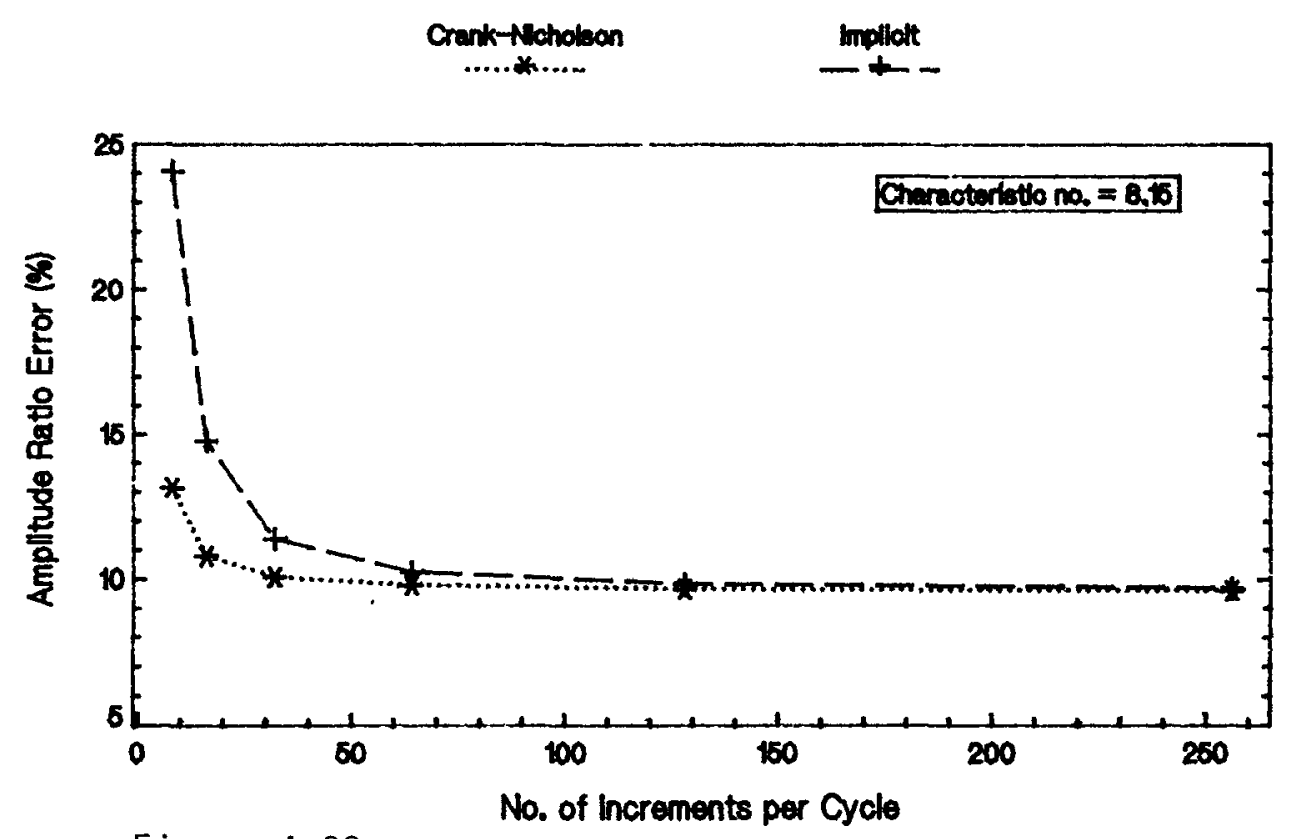

Figure 4.22

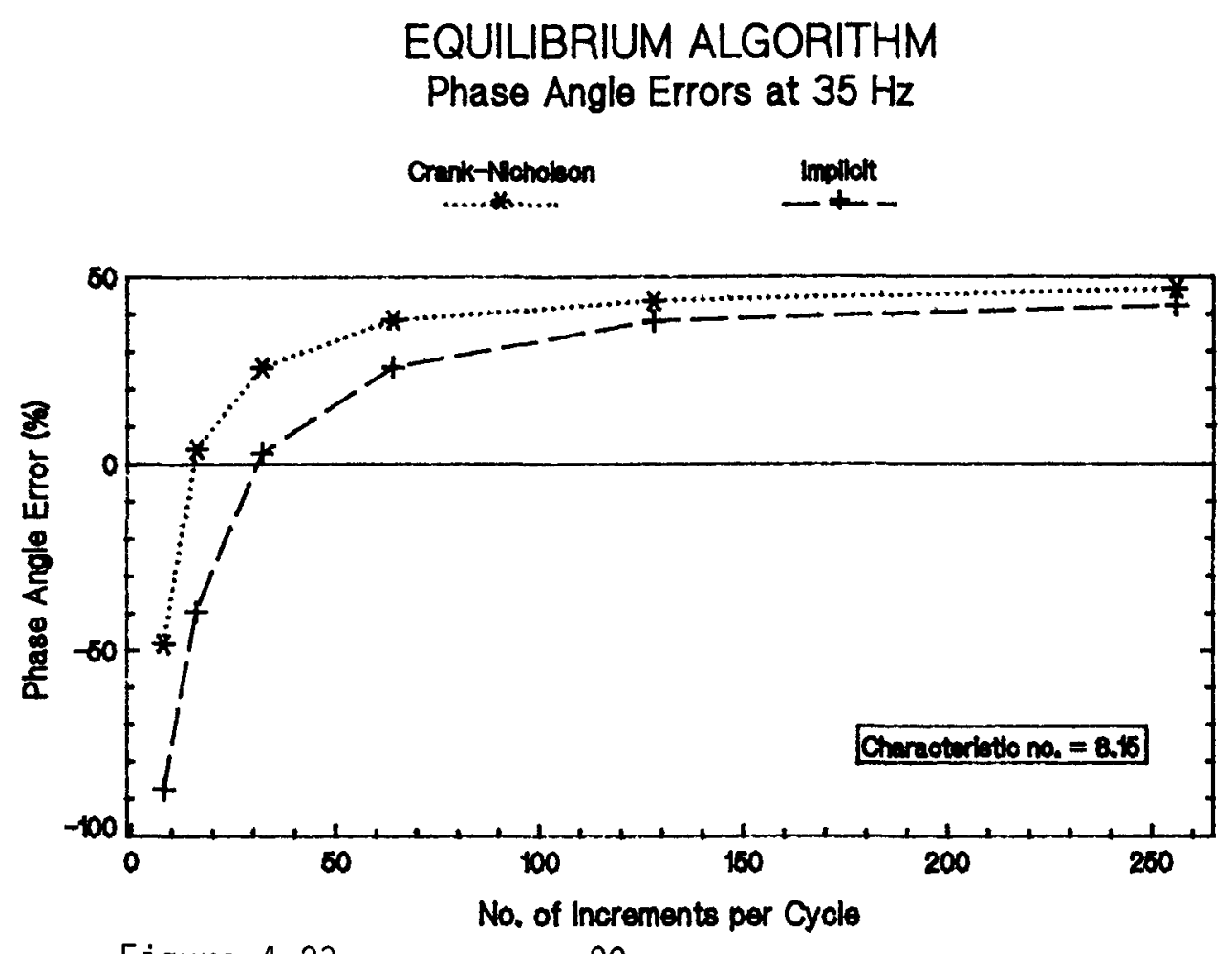

Figure 4.23 90 
EQUILIBRIUM ALGORITHM

Amplitude Ratio Comparison at $21.5 \mathrm{~Hz}$

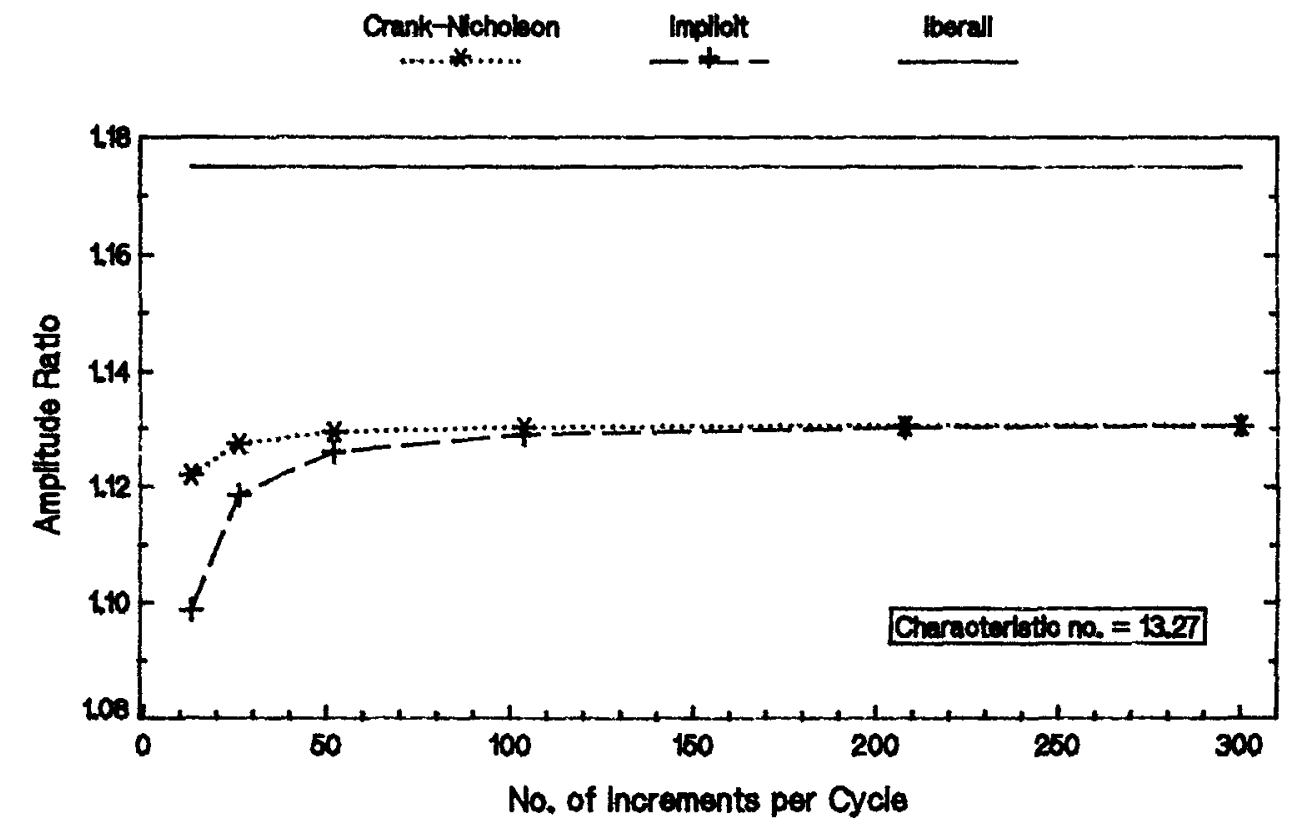

Figure 4.24

EQUILIBRIUM ALGORITHM

Phase Angle Comparison at $21.5 \mathrm{~Hz}$

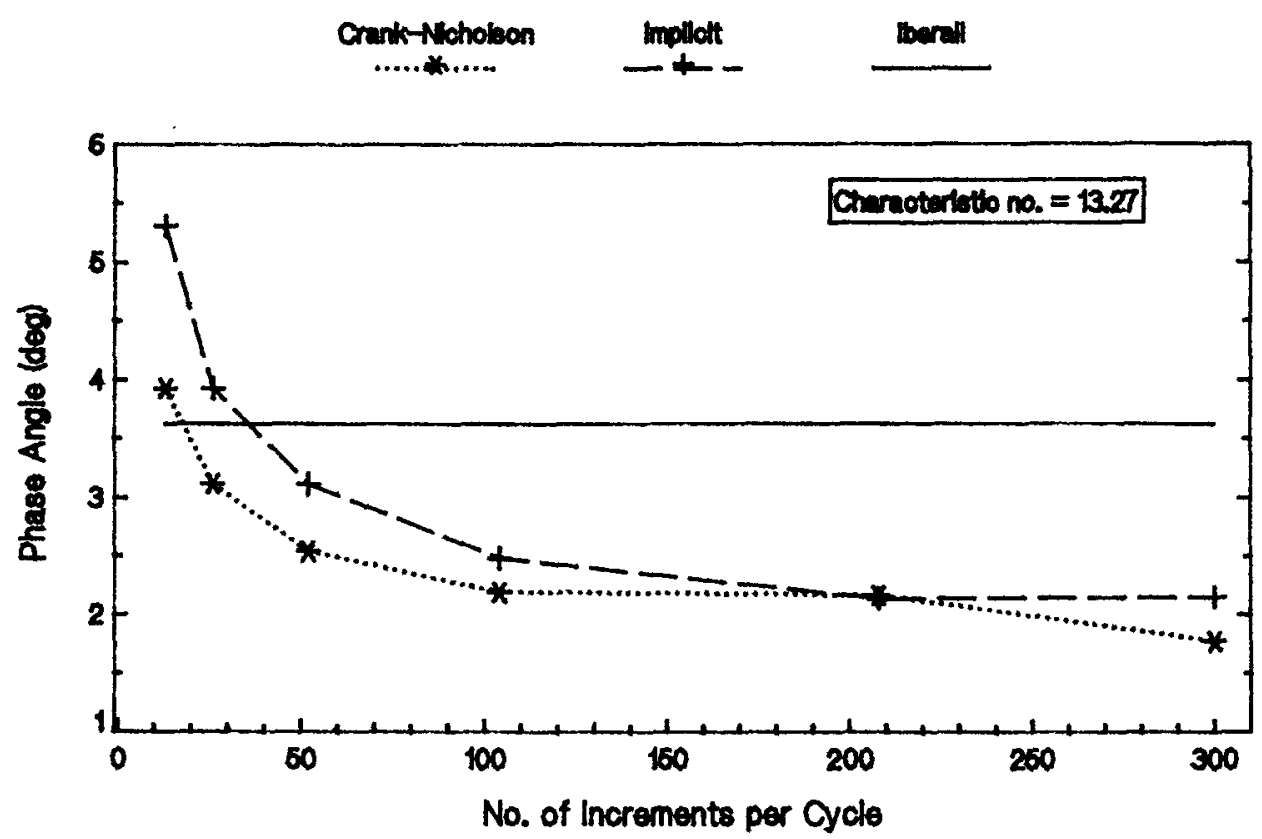

Figure 4.25 
EQUILIBRIUM ALGORITHM

Amplitude Ratio Errors at $21.5 \mathrm{~Hz}$

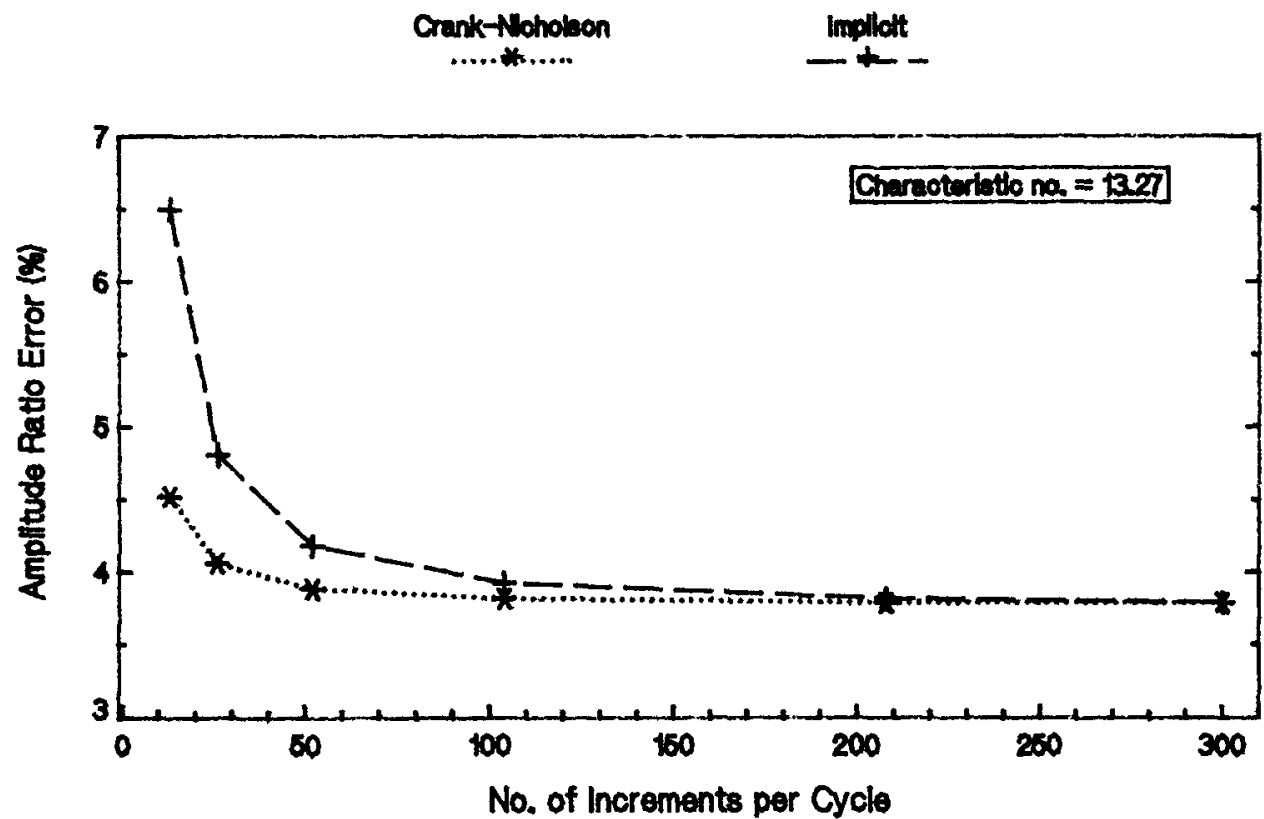

Figure 4.26

EQUILIBRIUM ALGORITHM

Phase Angle Errors at $21.5 \mathrm{~Hz}$

Crenis-Noholion

.............

ingliat

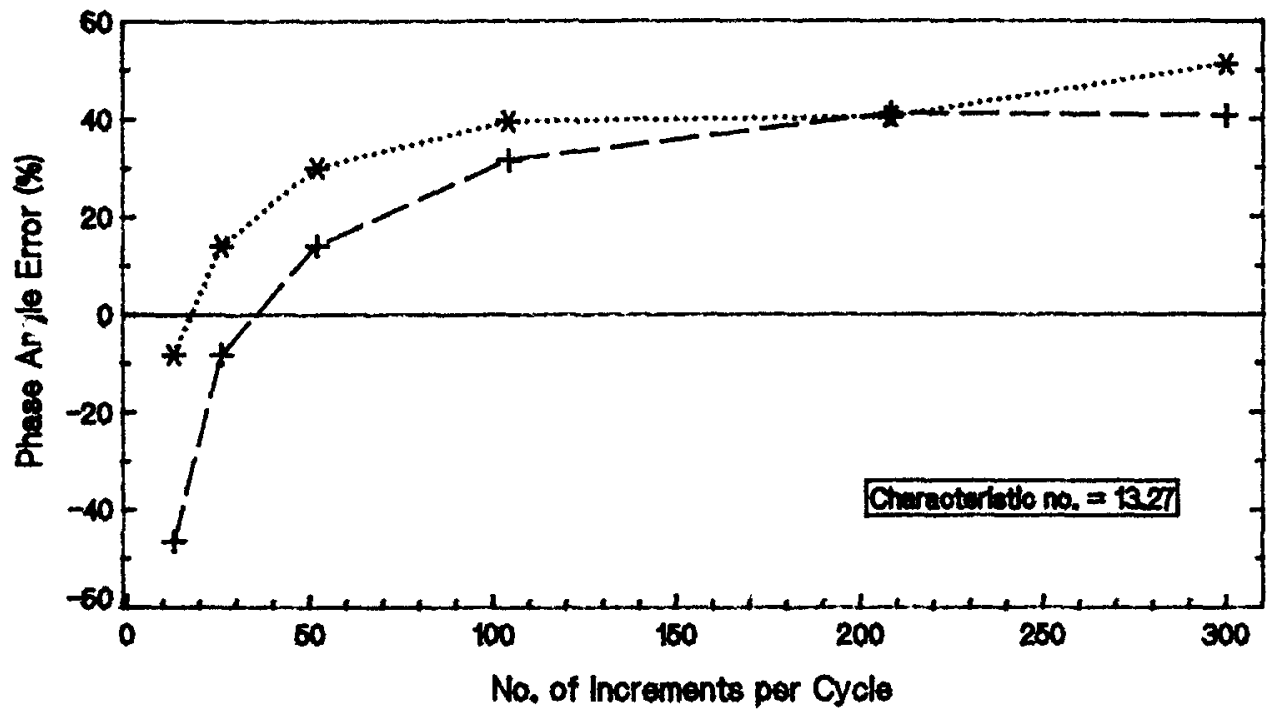

Figure 4.27 


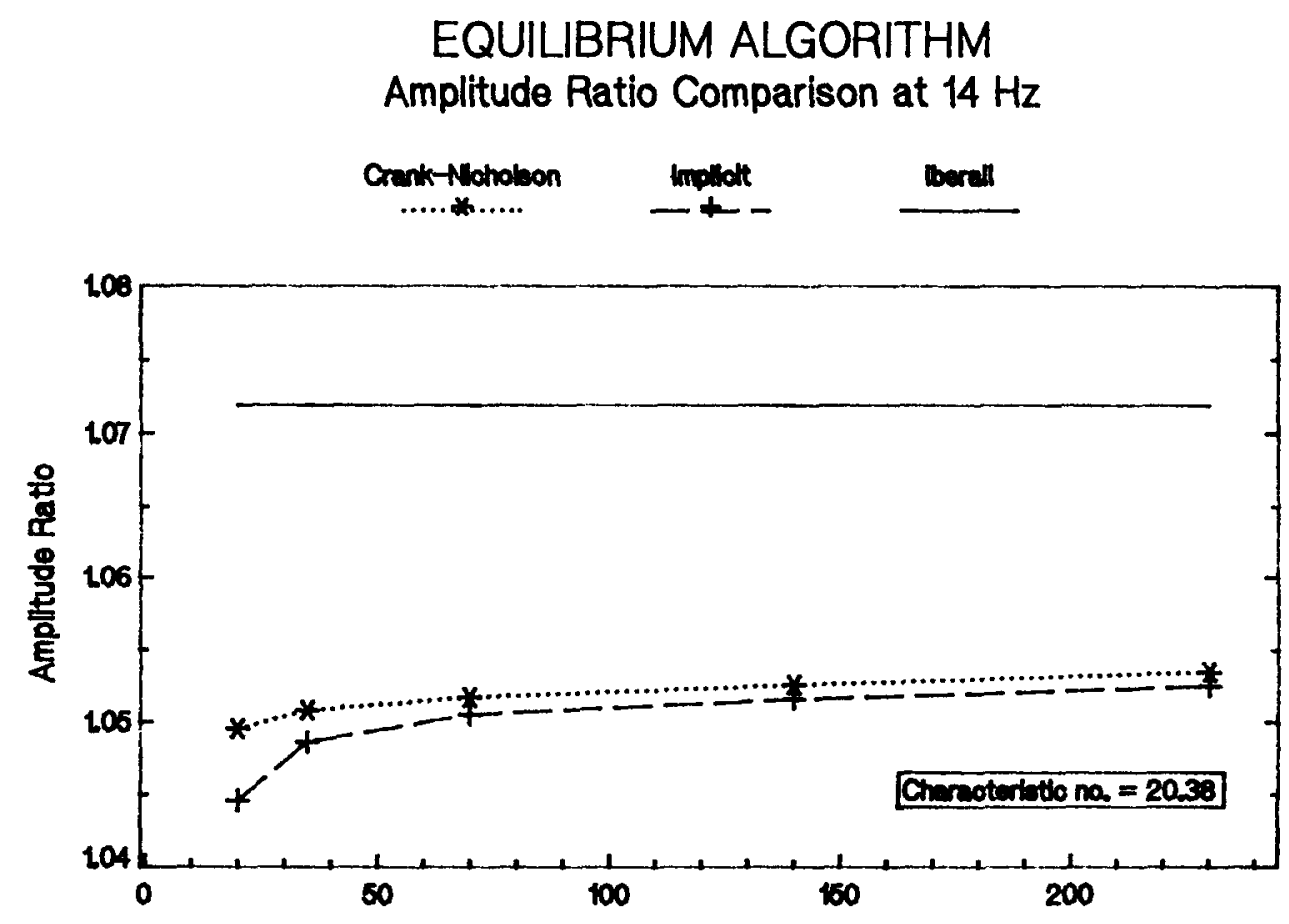

Figure 4.28

No. of increments per Cycle

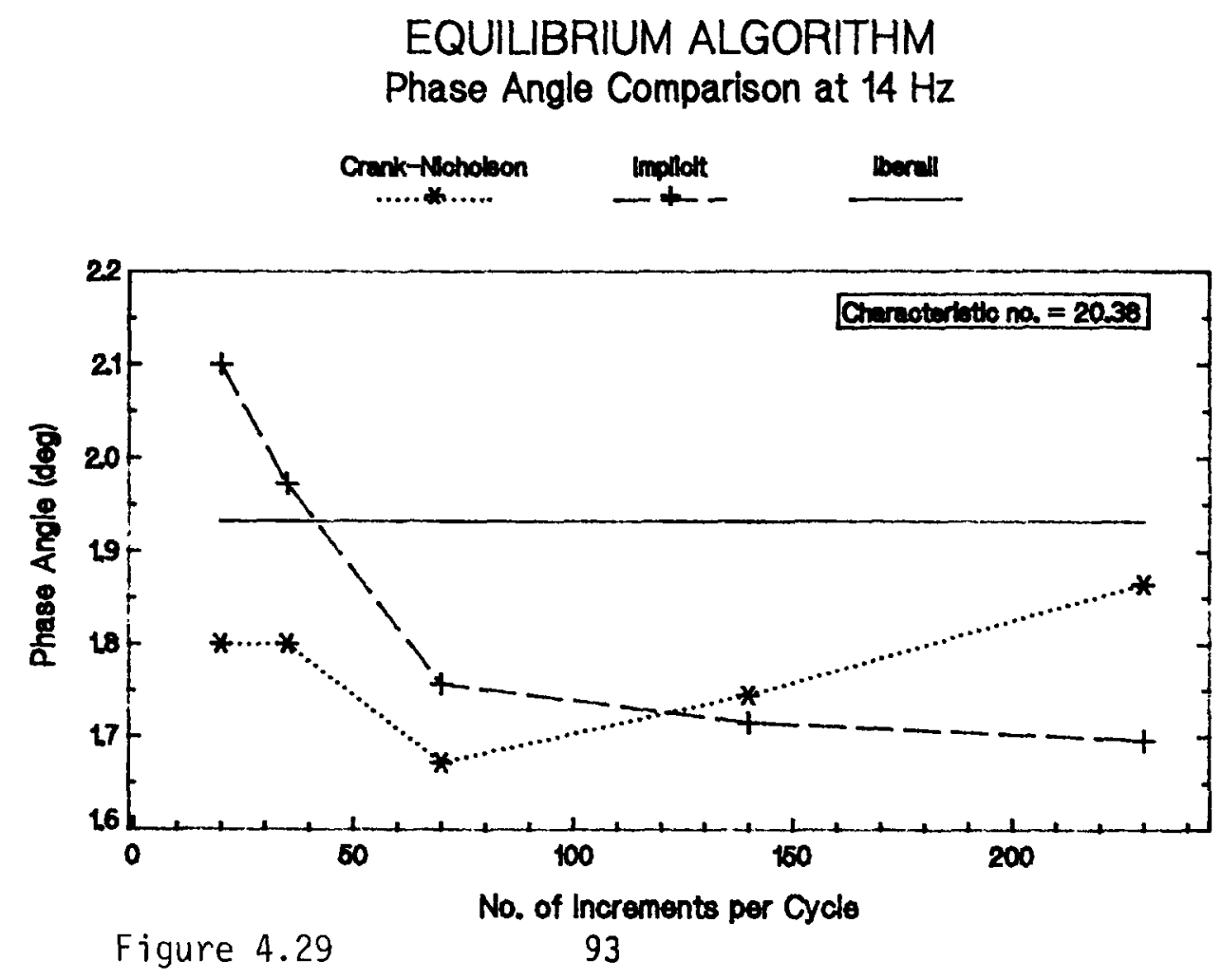


EQUILIBRIUM ALGORITHM

Amplitude Ratio Errors at $14 \mathrm{~Hz}$

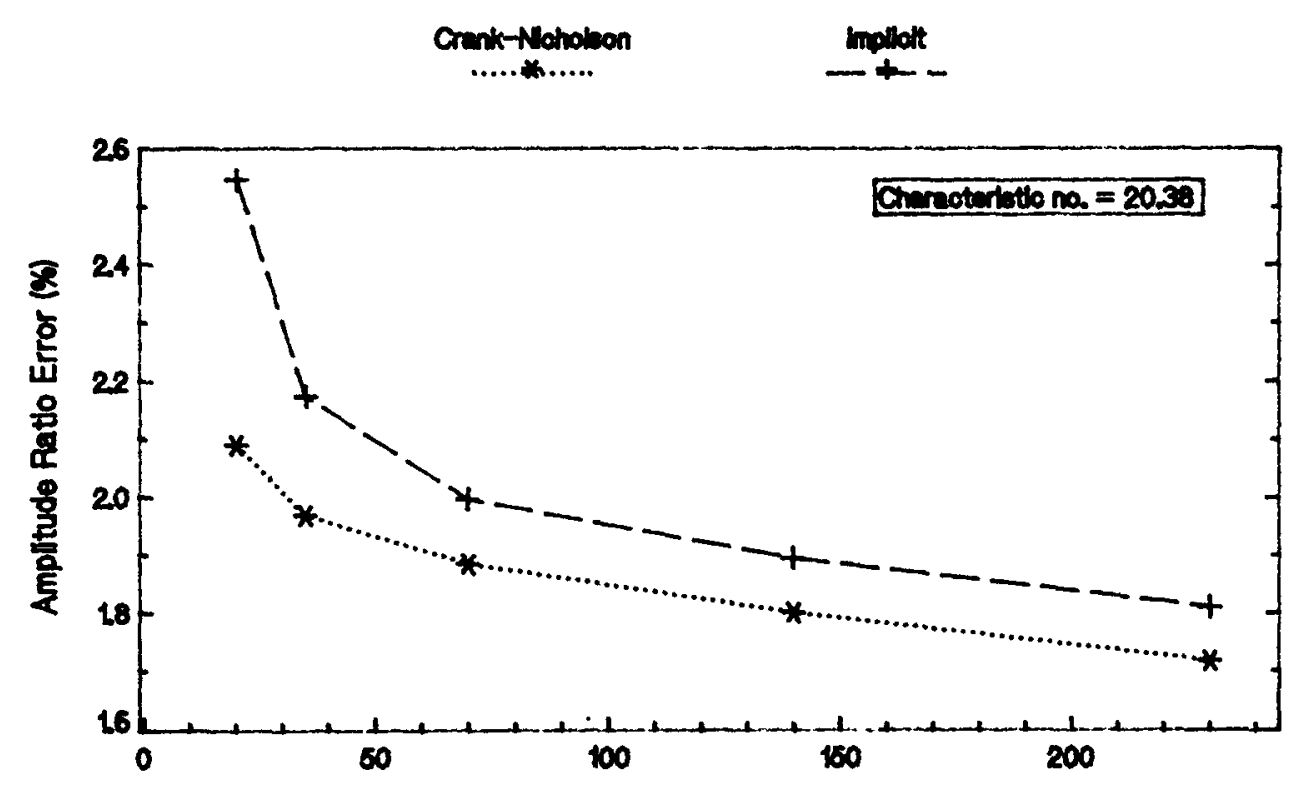

No. of increments per Cycle

Figure 4.30

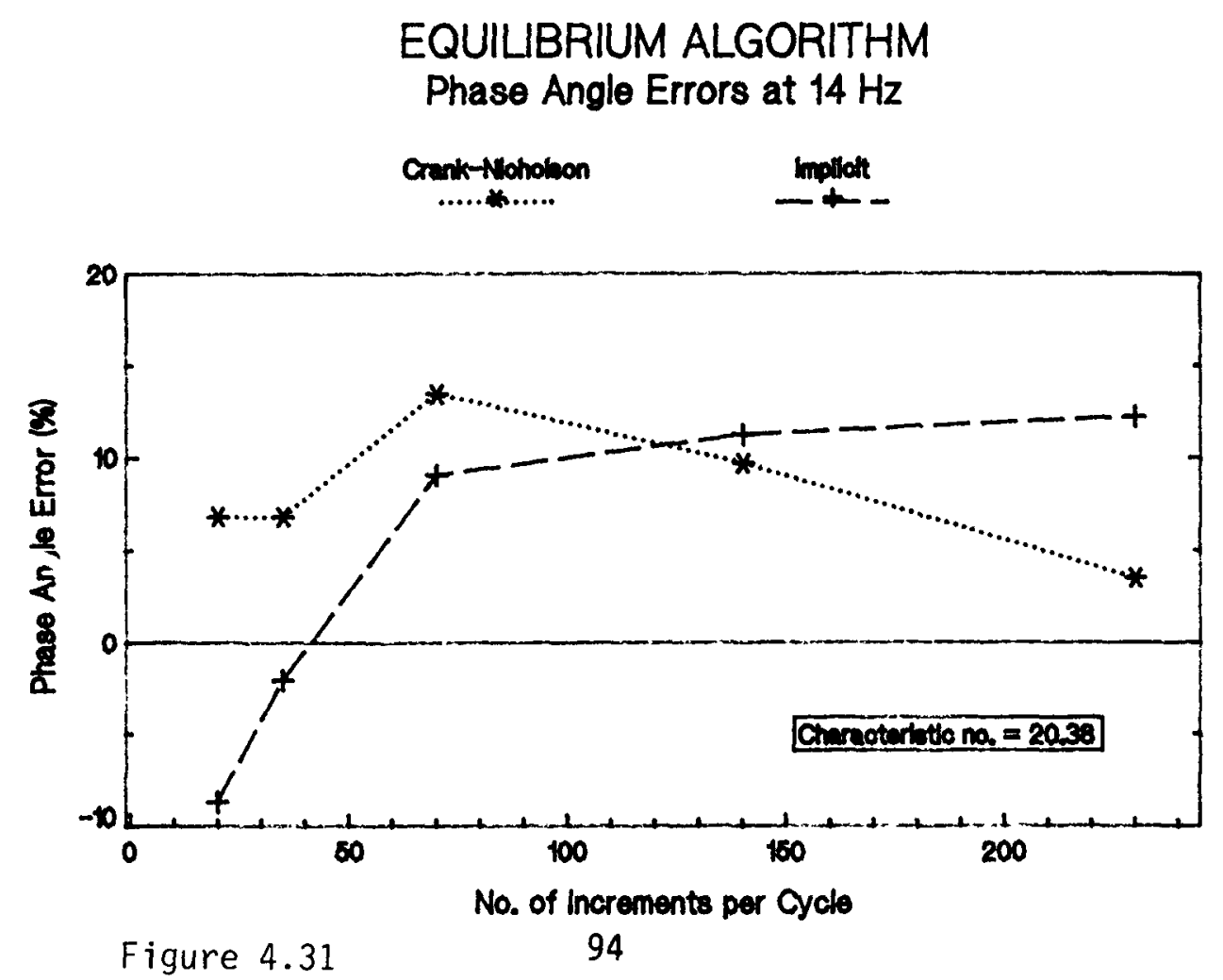


In general terms, these phenomena confirm those observed in section 4.5, namely, that under assumed equilibrium information propagation conditions at low characteristic numbers, the errors in modelling information propagation using the equilibrium hypothesis produce incorrectly simulated phase angles while reducing the simulated amplitude ratio errors. The fact that this behavior is exhibited by both the $\mathrm{CN}-\mathrm{s}$ and I-s indicates the existence of a physical effect that is not properly modelled by either the CN-s or the I-s techniques owing to their numerical limitations.

\subsection{CONCLUSION}

Essentially it seems that numerical integration errors are a reasonable hypothesis for describing the limited range of applicability of the one-step implicit application of the UPD algorithm. This conclusion is also consistent with the apparent success of the GLIMPS code in duplicating Watts' amplitude ratio data for $N_{c h}<8.15$. However, a generalised implementation of a multistep implicit method would have to be developed and applied to the transmission line to give this assertion better credence.

A further complicating issue arises from the difference between the UPD/equilibrium and GLIMPS codes in the numerical implementation of the conservation balances. The former uses a pressure-linked algorithm (with the limitations discussed in section 4.6) while the latter does not. This poses the question as to whether a pressure-linked algorithm is valid at low characteristic numbers or, for that matter, whether it is valid for gasdynamic flows at all. Hence, there is a possibility that such a lack of validity may synergistically or in isolation account for the behavior of the UPD/equilibrium simulations when applied to the SPDE as a particular case. In general, such behavior has not been observed (Go87) when, apparently, the characteristic numbers have been large enough (greater than 60 ) to make the assumption of infinite information propagation reasonable.

As a practical consequence of this work, it is recommended that the SPDE one-dimensional simulation code be converted from an I-s to a CN-s. This should provide a better margin of safety between the validity limit of the $\mathrm{CN}$ $s$ and the operating characteristic number of the SPDE.

In terms of the two-dimensional SPDE code, it may be argued that even the CN-s UPD simulation is inadequate, particularly in terms of implementing workable turbulence models. This arises because of the apparent conflict between the smaller time steps required for these models and the larger time steps required for modelling information propagation using a first order implicit algorithm. Thus, absent the availability of viable alternatives (such as PDS or multi-step implicit methods) it seems appropriate at this stage to concentrate on the turbulence modelling in the two-dimensional code using the equilibrium hypothesis and simply acknowledge the resulting existence of information propagation errors at low characteristic numbers. 


\subsection{PROGNOSIS}

In view of the wealth of information gleaned from comparing the simulation codes against Iberall's analysis, it is recommended that such a comparison be adopted as a universal validity check for stirling machine simulation codes.

The development of either a PDS or a generalised multi-step implicit method (with the latter alternative appearing preferable at this stage) for Stirling machine simulation as well as an independent investigation of the validity of pressure-linking both appear to be worthwhile future research activities. 
S I M ULA T I O N O F THE MECHANI C A L

E N G I N E E R I N

\subsection{INTRODUCTION}

The philosophy guiding the simulation of the mechanical engineering test rig (METR) was to provide a means of using its empirical data in a generalised simulation of Stirling cycle machines in both one and two dimensions. The following methodology was conceptualised for accomplishing this goal:

a. Validate a system-based simulation of the METR including a twodimensional description of the test section. The primary goal of this exercise was to define a turbulence model that will enable the experimental data to be matched in the context of a system simulation that includes an accurate description of actual oscillating flow boundary conditions.

b. Based on the validated two-dimensional code predictions, develop a set of friction factor and heat transfer correlations which may be used in a one-dimensional simulation.

c. Compare the one-dimensional predictions directly against the empirical data and, if necessary, refine the correlations.

d. Test the one- and two-dimensional simulations against available experimental data for actual Stirling hardware, in this case, the SPDE.

The work described in this chapter represents the progress made in accomplishing these tasks. The simulation activities listed fundamentally depend on the availability of METR experimental data of sufficiently high quality. For this reason, at the termination of the project, sufficient experimental data were available only to make a preliminary attempt at completing task a (see chapter 1 ).

Two sets of experimental data are available. The first set is used to define a baseline simulation case against which the effect of turbulence models may be measured. This baseline excludes all turbulence modelling from the two-dimensional components (that is, the test section is described twodimensionally in purely laminar terms) and uses standard Kays and London (KL64) steady-state friction factor and heat transfer correlations for all the one-dimensional components. 
A further objective of the baseline simulations was to provide feedback to Simon and Seume on refinements to the METR instrumentation system and, in particular, on which additional measurements were necessary in order to achieve adequate simulation validation. In this context, it must be noted that the baseline data provided by Seume and Simon are of a preliminary nature and were intended not only to 'shake down' the METR instrumentation system but also to refine the mechanics of the raw experimental data transfer and processing. These data are acknowledged to be unsuitable for turbulence model development and simulation validation (Se88).

The second set of data, which is nominally of sufficient quality for simulation development and validation, consists only of half a cycle of data at one probe location. Unfortunately, this data set is really too small and insufficiently free of systematic errors to allow a truly meaningful attempt at validating the turbulence model developed. Hence the model proposed is of a tentative nature only and, therefore, fulfillment of the overall goal as well as the stated objective (of completely validating the turbulence model developed) must await the availability of further experimental data.

\subsection{DESCRIPTION OF THE TEST RIG}

A schematic of the METR as simulated is shown in figure 5.1. The rig consists of a combination of seven components arranged axially in a symmetrical pattern about the test section midpoint. A piston/cylinder is located on the left-hand end of the rig while the right-hand end is open to the atmosphere. The universal sign convention adopted is that the positive direction is toward the right-hand or open end of the rig. The METR is simulated as operating with purely isothermal boundaries since no heat transfer measurements are as yet available. Hence all the boundary temperatures are kept constant at the ambient value.

The individual rig component descriptions used in the baseline simulations correspond to those listed in table 5.1. This table is the output of a geometry definition computer program which enables the rig dimensions to be arbitrarily altered in terms of the defined rig components and component sequence. The program may be readily modified to include new component definitions as well as different sequences of defined components. Table 5.1 reveals that care has been taken to define those minor details of the rig geometry that are judged to have a more than negligible effect on the fluid flow.

The spatial discretisations employed (table 5.1) conform to those established as suitable for an integral description of Stirling machine fluid dynamics (Go87). The $11 \times 8$ mesh used in the test section for the twodimensional description ( 21 axial control volumes are used in the onedimensional description) is a compromise between adequate spatial resolution and the available computing power. It should be noted that 'adequate' spatial resolution for an integral (or discrete volume) analysis is very different from that required for a differential analysis. In this context, increasing the spatial resolution toward differential levels would require the use of a 


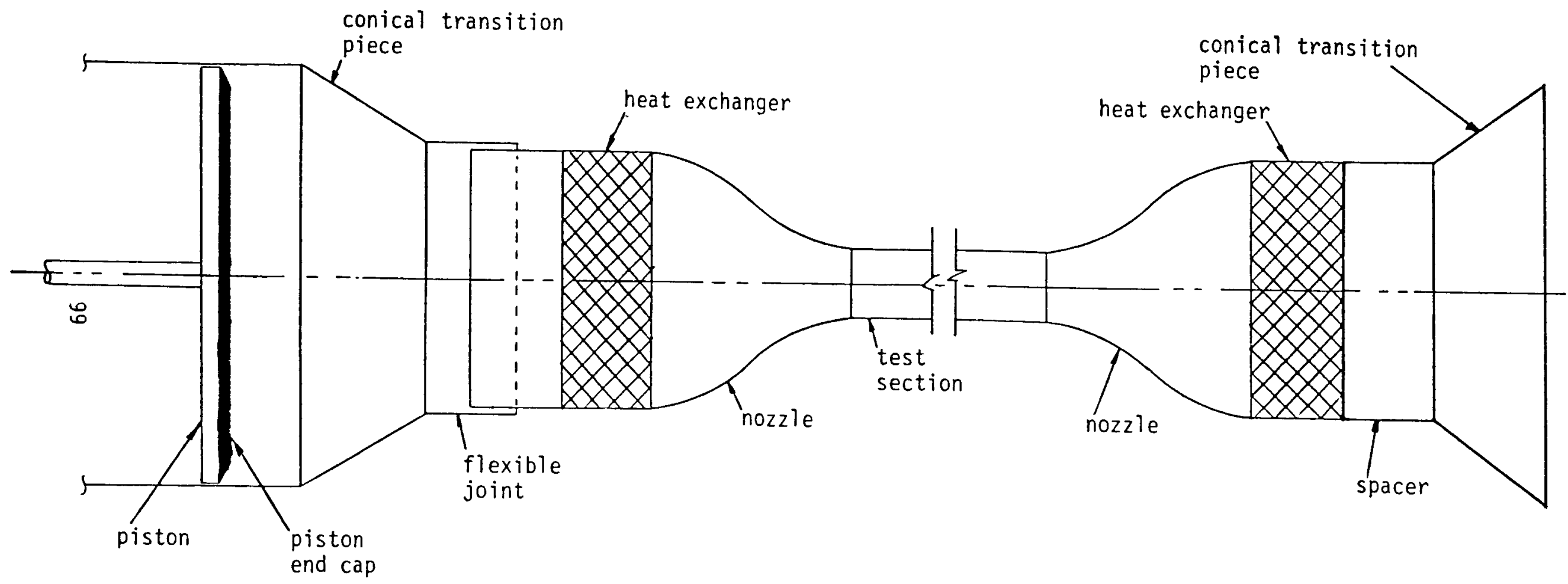

Figure 5.1 Schematic of Mechanical Engineering Test Rig 
Table 5.1 METR baseline simulation input parameter set.

\begin{tabular}{|c|c|}
\hline $\begin{array}{l}\text { Expansion Space } \\
\text { Piston stroke (mm) } \\
\text { Piston end cap thickness (mm) } \\
\text { Piston end cap taper (deg) } \\
\text { Clearance length at piston TDC (mm) } \\
\text { Cylinder diameter (mm) } \\
\text { Angular velocity (rpm) } \\
\end{array}$ & $\begin{array}{l}355.6 \\
6.0 \\
30.0 \\
3.1750 \\
355.6 \\
11.25 \\
\end{array}$ \\
\hline $\begin{array}{l}\text { Conical Transition Piece } \\
\text { Axial length(mm) } \\
\text { Cylinder mating diameter }(\mathrm{mm}) \\
\text { Flexible joint mating diameter }(\mathrm{mm}) \\
\text { \# of control volumes } \\
\end{array}$ & $\begin{array}{l}212.725 \\
355.6 \\
127.0 \\
\quad 3 \\
\end{array}$ \\
\hline $\begin{array}{l}\text { Flexible Joint } \\
\text { Cylinder side axial length(mm) } \\
\text { Cylinder side diameter(mm) } \\
\text { Cylinder side \# of control volumes } \\
\text { Nozzle side axial length(mm) } \\
\text { Nozzle side diameter }(\mathrm{mm}) \\
\text { Nozzle side \# of control volumes } \\
\end{array}$ & $\begin{array}{r}21.0 \\
152.0 \\
2 \\
63.0 \\
139.7 \\
2 \\
\end{array}$ \\
\hline $\begin{array}{l}\text { Spacer } \\
\text { Axial length(mm) } \\
\text { Cylinder side flange length }(\mathrm{mm}) \\
\text { Nozzle side flange length(mm) } \\
\text { Diameter(mm) } \\
\text { Cylinder side flange diameter }(\mathrm{mm}) \\
\text { Nozzle side flange diameter }(\mathrm{mm}) \\
\text { \# of control volumes } \\
\end{array}$ & $\begin{array}{r}63.5 \\
3.175 \\
6.35 \\
139.7 \\
127.0 \\
127.0 \\
3\end{array}$ \\
\hline $\begin{array}{l}\text { Heat Exchanger } \\
\text { Axial length }(\mathrm{mm}) \\
\text { Cylinder side flange length }(\mathrm{mm}) \\
\text { Nozzle side flange length }(\mathrm{mm}) \\
\text { Casing diameter }(\mathrm{mm}) \\
\text { Hydraulic diameter }(\mathrm{mm}) \\
\text { Flow area ratio } \\
\text { \# of control volumes }\end{array}$ & $\begin{aligned} 50.8 \\
9.525 \\
12.7 \\
127.0 \\
2.1824 \\
.556 \\
4\end{aligned}$ \\
\hline $\begin{array}{l}\text { Nozzle } \\
\text { Size (1=small, } 2=\text { =large) } \\
\text { \# of half-nozzle control vols }\end{array}$ & $\begin{array}{l}1 \\
3 \\
\end{array}$ \\
\hline $\begin{array}{l}\text { Test Section } \\
\text { External diameter(mm) } \\
\text { Axial length }(\mathrm{mm}) \\
\text { \# of axial control volumes (4 minimum) } \\
\text { \# of radial control volumes (2 minimum) } \\
\text { Wall conductivity }(\mathrm{W} / \mathrm{m}-\mathrm{K}) \\
\text { Wall density }\left(\mathrm{kg} / \mathrm{m}^{\wedge} 3\right) \\
\text { Wall specific heat capacity }(\mathrm{J} / \mathrm{kg}-\mathrm{K}) \\
\end{array}$ & $\begin{array}{lc}: & 50.8 \\
: & 2609.1 \\
: & 21(1-\mathrm{d}) \\
& 11(2-\mathrm{d}) \\
: & 8 \\
: & 1.0211 \\
: & 2466.84 \\
: \quad 753.624 \\
\end{array}$ \\
\hline $\begin{array}{l}\text { Oorking Fluid } \\
\text { Working fluid ( } 1=\text { Air } \quad 2=\text { Helium, } 3=\mathrm{Hydr}\end{array}$ & \\
\hline
\end{tabular}


supercomputer, for which no funds were available. Hence, determining the impact of radically changing the test section spatial resolution must of necessity be neglected here.

\subsection{BASELINE CASE SIMULATION APPLICATION}

The one- and two-dimensional simulation codes used to model the METR are in essence identical to those used for the SPDE. The codes are rearranged to conform to the geometry of the METR by excluding the regenerator and the compression space and by reconfiguring the connecting ductwork. The twodimensional heater module is adjusted to represent the geometry of the single tube test section. The pseudo-two-dimensional heater boundary condition used at the expansion space side of the SPDE heater is replaced with a onedimensional boundary condition appropriate to a nozzle (with an axially varying flow area).

In converting the SPDE codes to the METR geometry, the following revisions to the numerical algorithm have been implemented:

The energy balance reporting error has been corrected yielding typical energy balance percentage errors of order $10^{-2}$ or less (compared with errors of order $10^{\circ}$ previously).

- Computationally more efficient discretisation methods have been developed for calculating the dissipation and reversible thermal/kinetic energy conversion terms.

- Provision has been made for describing control volumes that may be generated as bodies of revolution based on continuous functions (cones, paraboloids, etc.).

In order to fulfill the objective of generating a baseline laminar flow case for the two-dimensional simulation of the test section, the following adjustments to the code have been made:

The Reynolds stress tensor, turbulent heat flux and dissipation terms have been removed from the momentum and thermal energy equations, respectively (equations 3.3 ).

- Mass flux fluctuations generated by the iterative integration algorithm are numerically filtered out (such fluctuations are a manifestation of a chaos model implicitly embedded in the algorithm). A radially polarised cut-off amplitude of $10^{-1}$ $\mathrm{kg} / \mathrm{m}^{2} . \mathrm{s}$ is imposed, which has the effect of removing all the turbulence with RMS amplitudes larger than the cut-off amplitude; the smaller scales remain. 


\subsection{BASELINE CASE RESULTS}

The primary focus of the following discussion is directed towards a comparison of the available experimental data with its simulated counterparts. This does not constitute a rigorous validation but does serve to expose those areas requiring further experimental and analytic attention.

\subsubsection{Experimental Data}

The experimental data set made available by Seume and Simon for validating the simulation consists of:

bulk mean velocities, that is, area-weighted, one-dimensional flow velocities

- axial ensemble-averaged mean velocities and root-mean-square (RMS) velocity fluctuations at seven locations on two radial planes (at the midpoint and toward the cylinder end of the test section)

These data were gleaned for the parametric conditions shown in table 5.2.

Table 5.2 Parameters for the baseline case experimental data.

\begin{tabular}{lcc}
\hline Date & $\begin{array}{c}\text { Engine Speed } \\
(\mathrm{rpm})\end{array}$ & $\begin{array}{c}\text { Probe Position (mm) } \\
\text { (relative to cylinder end) }\end{array}$ \\
\hline $4 / 22 / 88$ & 11.3 & 1304 (midpoint) \\
$4 / 24 / 88$ & 11.3 & 609 \\
$4 / 26 / 88$ & 33.5 & 1304 (midpoint) \\
\hline
\end{tabular}

The data set supplied is not entirely adequate for simulation validation purposes since several key data are missing. Specifically, the missing data include:

- ambient pressure and temperature conditions.

- transient leakage flow rates past the piston

The actual rig geometry is considered to be adequately represented by the description encompassed within table 5.1 with the usual uncertainties present in the fin surface geometry assumed for the heat exchangers. On the advice of Seume, a standard Kays and London (KL64) louvered plate-fin surface designated ' $1 / 4(\mathrm{~b})-11.1$ ' has been assumed together with its steady-state friction factor and heat transfer coefficient correlations.

In the absence of any cylinder leakage data, the simulation is performed on the basis that no leakage occurs in the cylinder. This may produce discrepancies between the experimental and simulated bulk flow velocities. 
Should cylinder leakage actually occur, then the discrepancies may be used as an a posteriori means of assessing what leakage should be incorporated into the simulation to match the experimental data.

The unavailability of measured ambient temperatures and pressures is of much greater significance because the rig operates as an open system. Furthermore, since the two tests at a constant rig speed of $11.3 \mathrm{rpm}$ were performed on different days, local weather conditions are such that 7 to $10 \%$ variations in barometric pressure are possible between tests. This can produce significant discrepancies in comparing the simulation and experimental data. Nevertheless, for the purpose of carrying out the validation exercise, it is necessary to assume an ambient temperature and pressure. Values of $26^{\circ} \mathrm{C}$ and 0.98274 bar, respectively, were chosen, these being typical averages for the days during which the tests were conducted.

\section{4 .2 Comparison of System Results at $11.3 \mathrm{rpm}$}

Figure 5.2 shows a comparison of the experimental bulk velocity profiles at $11.3 \mathrm{rpm}$ with those simulated in one dimension. The difference between the profiles simulated at probe positions of 609 and $1304 \mathrm{~mm}$ are so small relative to the scale of the graph that the two profiles appear coincident. Figure 5.3 compares the experimental bulk velocity at $11.3 \mathrm{rpm}$ at the $1304 \mathrm{~mm}$ probe position with that produced by the two-dimensional simulation. There is no discernable difference between the one- and two-dimensional bulk velocity profiles at the $1304 \mathrm{~mm}$ probe position, which is also true for the system axial velocity profiles shown in figures 5.4 and 5.5 . Other than the difference in axial discretisation, the one- and two-dimensional simulated profiles are identical.

The phase of the simulated and measured profiles in figures 5.2 and 5.3 are in agreement, while the difference in amplitude of the profiles shows the extent of the piston leakage. This leakage is less than that expected by Seume and Simon, who used an ideal velocity profile to make their determination. The irregularity of the measured profiles at peak flow velocities is believed to be caused by adhesion of the piston seal to the cylinder wall and/or other mechanical effects.

of particular concern in figure 5.2, however, is the large difference in experimental amplitudes relative to the coincidence of those measured. When the flow is negative between $0^{\circ}$ and $180^{\circ}$ (by convention, flow toward the piston), the pressure at $1304 \mathrm{~mm}$ is greater than that at $609 \mathrm{~mm}$ since the pressure gradient is opposed to the shear forces acting on the flow. This is confirmed by the both the one- and two-dimensional simulated pressure profiles shown in figures 5.6 and 5.7. Hence, since the system is essentially isothermal over the test section (see figures 5.8 and 5.9 ), the density at $1304 \mathrm{~mm}$ is greater than that at $609 \mathrm{~mm}$. Hence, between about 80 and 100 degrees of crank angle when the flow acceleration is small or zero, in order to conserve mass, the velocity at $609 \mathrm{~mm}$ should be greater than that at 1304 $\mathrm{mm}$, which is indeed the case (also shown by the simulated data). 


\section{SIMULATED AND EXPERIMENTAL BULK VELOCITY PROFILES}

RIG SPEED: 11.3 rpm

\begin{tabular}{cccc}
$1-d$ simul. & $\begin{array}{c}\text { experiment } \\
609 \mathrm{~mm}\end{array}$ & $\begin{array}{c}1-d \text { simul. } \\
1304 \mathrm{~mm}\end{array}$ & $\begin{array}{c}\text { experiment } \\
1304 \mathrm{~mm}\end{array}$ \\
\hline
\end{tabular}

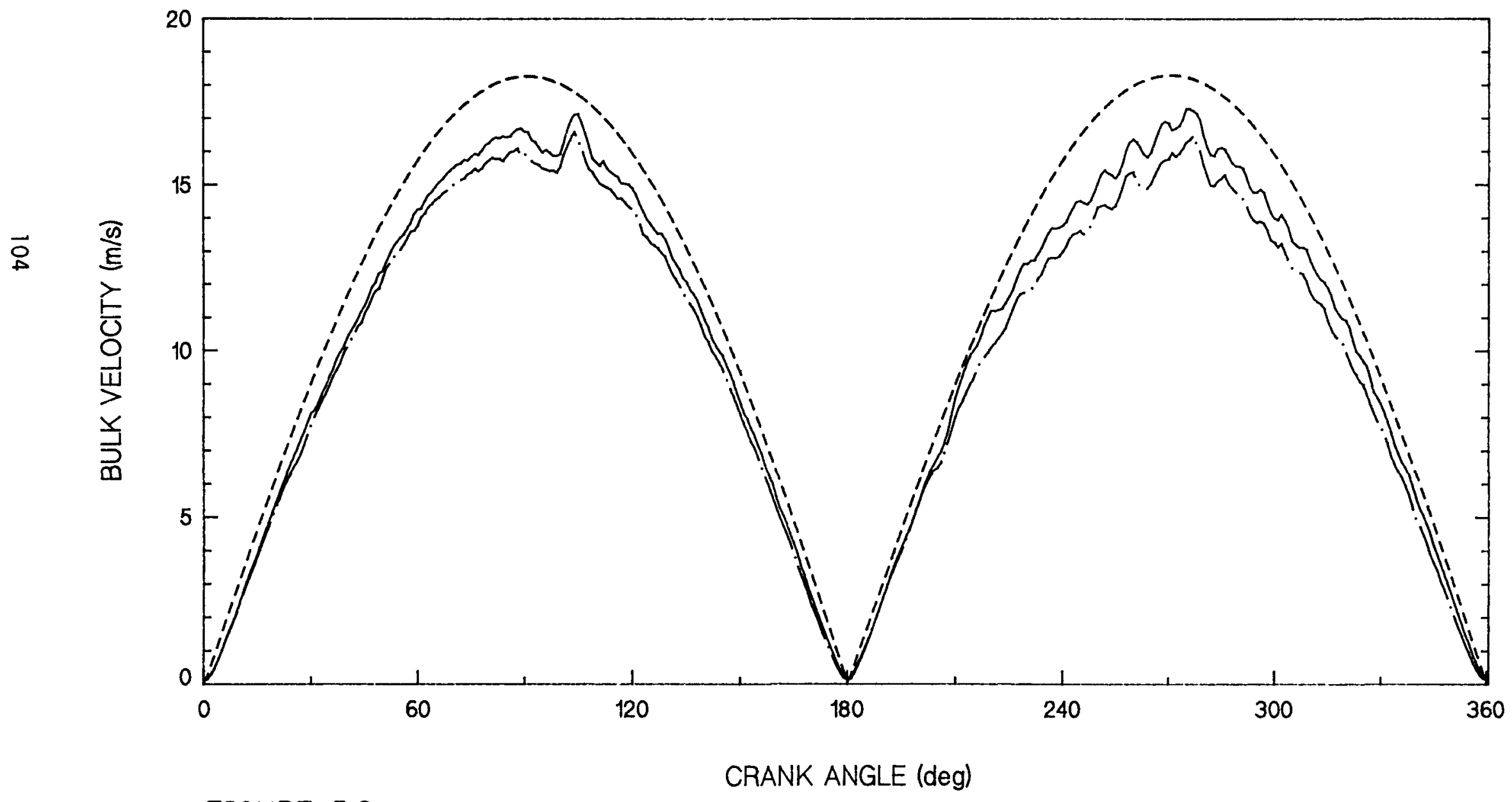

FIGURE 5.2 


\section{SIMULATED AND EXPERIMENTAL BULK VELOCITY PROFILES}

RIG SPEED: $11.3 \mathrm{rpm}$

2-d simul.

$1304 \mathrm{~mm}$

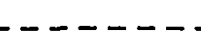

experiment

$1304 \mathrm{~mm}$

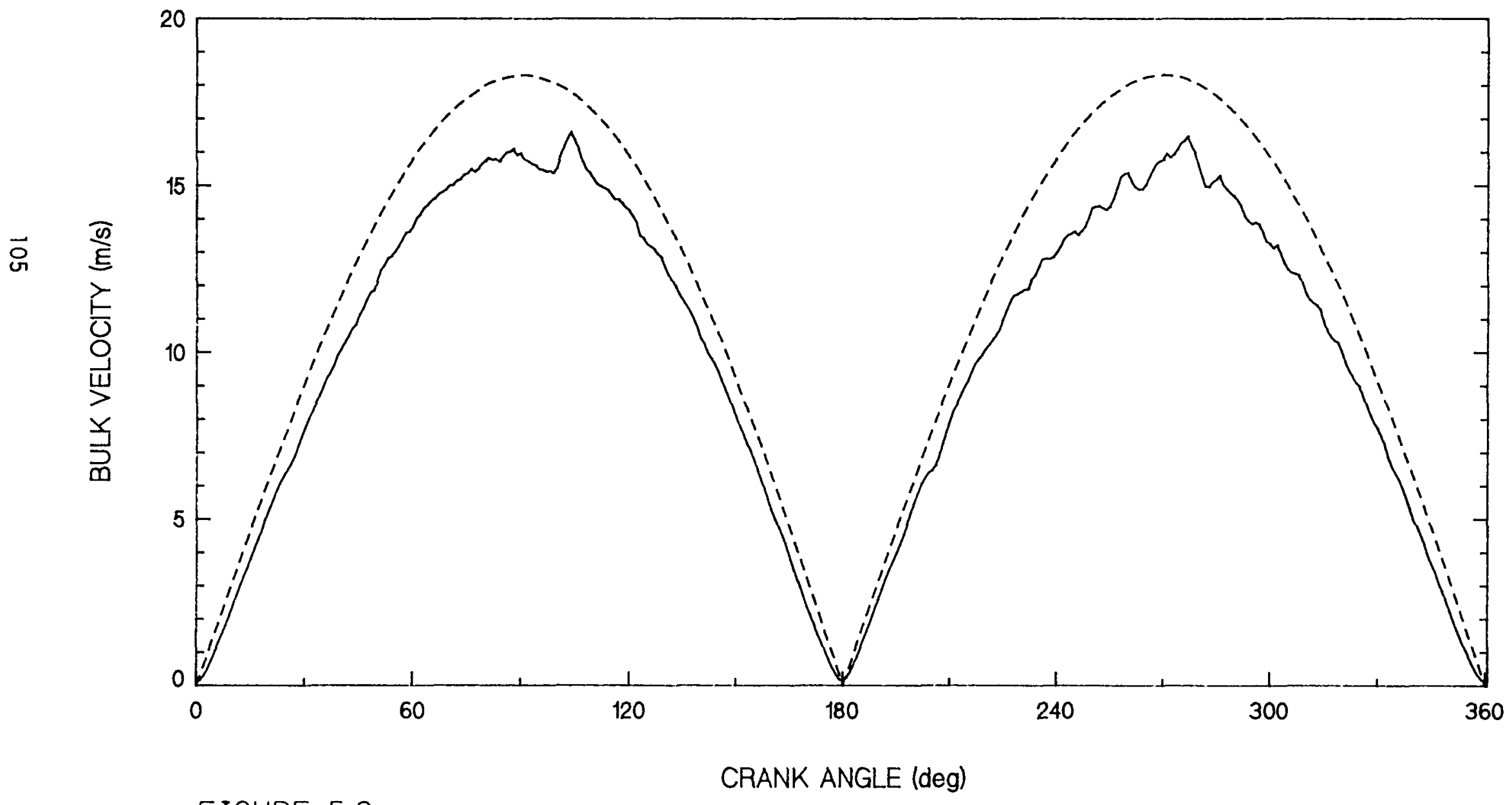

FIGURE 5.3 


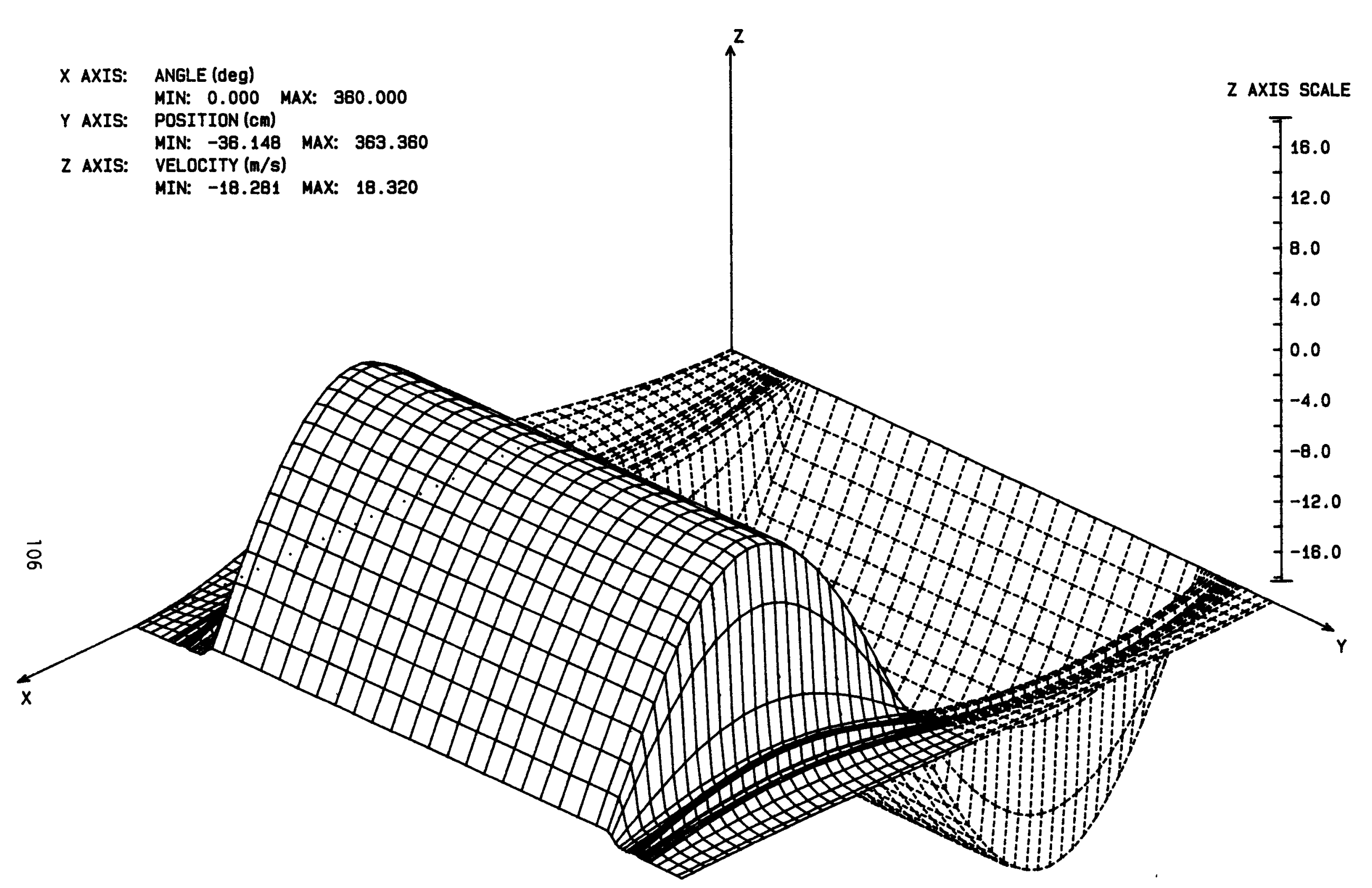

FIGURE 5.4 SYSTEM AXIAL VELOCITY PROFILE FROM 1-D CODE: $11.3 \mathrm{pmm}$ 


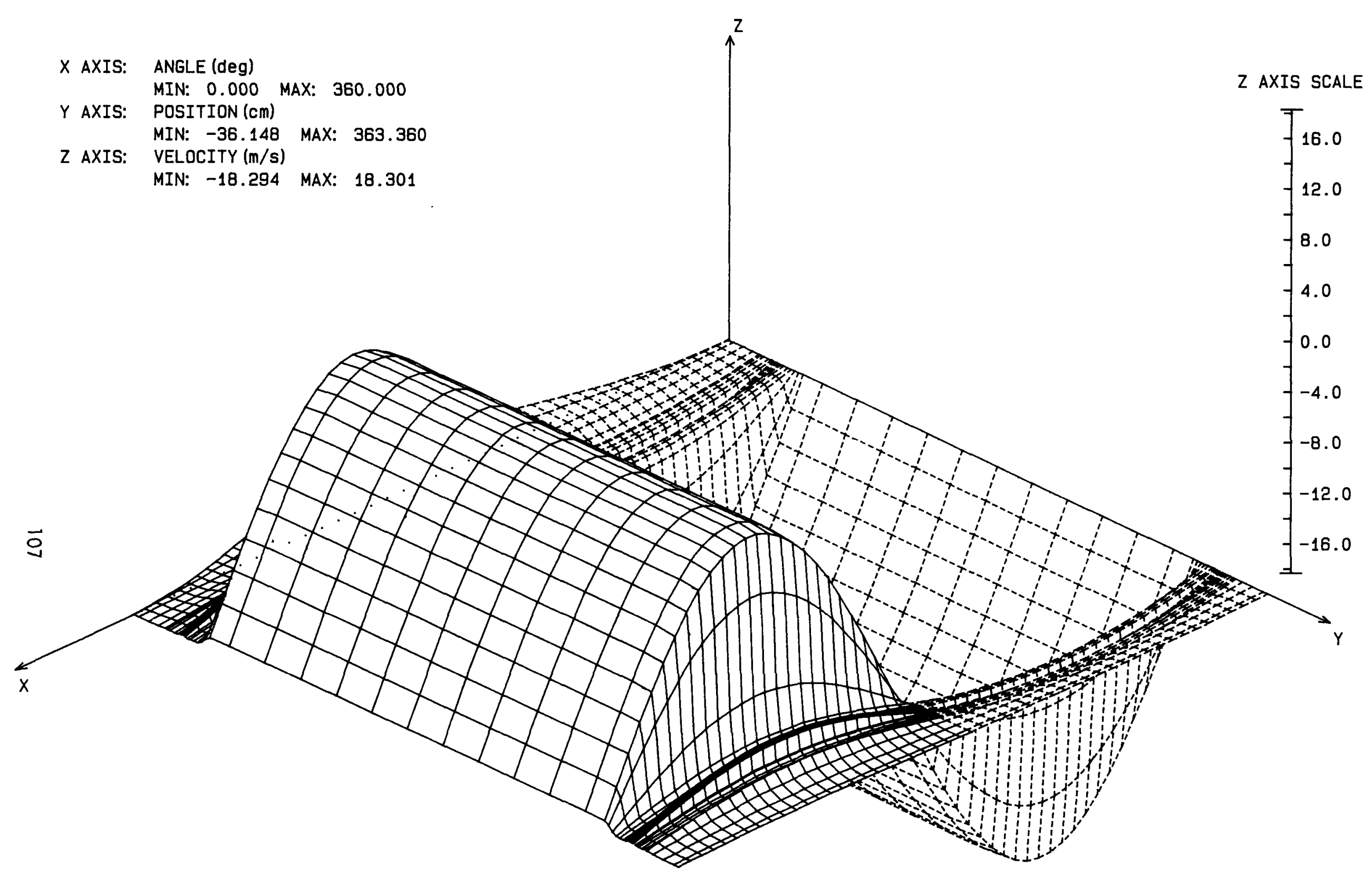

FIGURE 5.5 SYSTEM AXIAL VELOCITY PROFILE FROM 2-D CODE: $11.3 \mathrm{pmm}$ 

$X$ AXIS: ANGLE (deg)
MIN: 0.000 MAX: 360.000
$Y$ AXIS: POSITION (cm)
MIN: -36.148 MAX: 363.360
$Z$ AXIS: PRESSURE (bar)
MIN: 0.977 MAX: 0.987

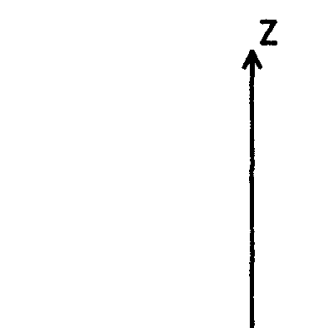

$\vec{\infty}$

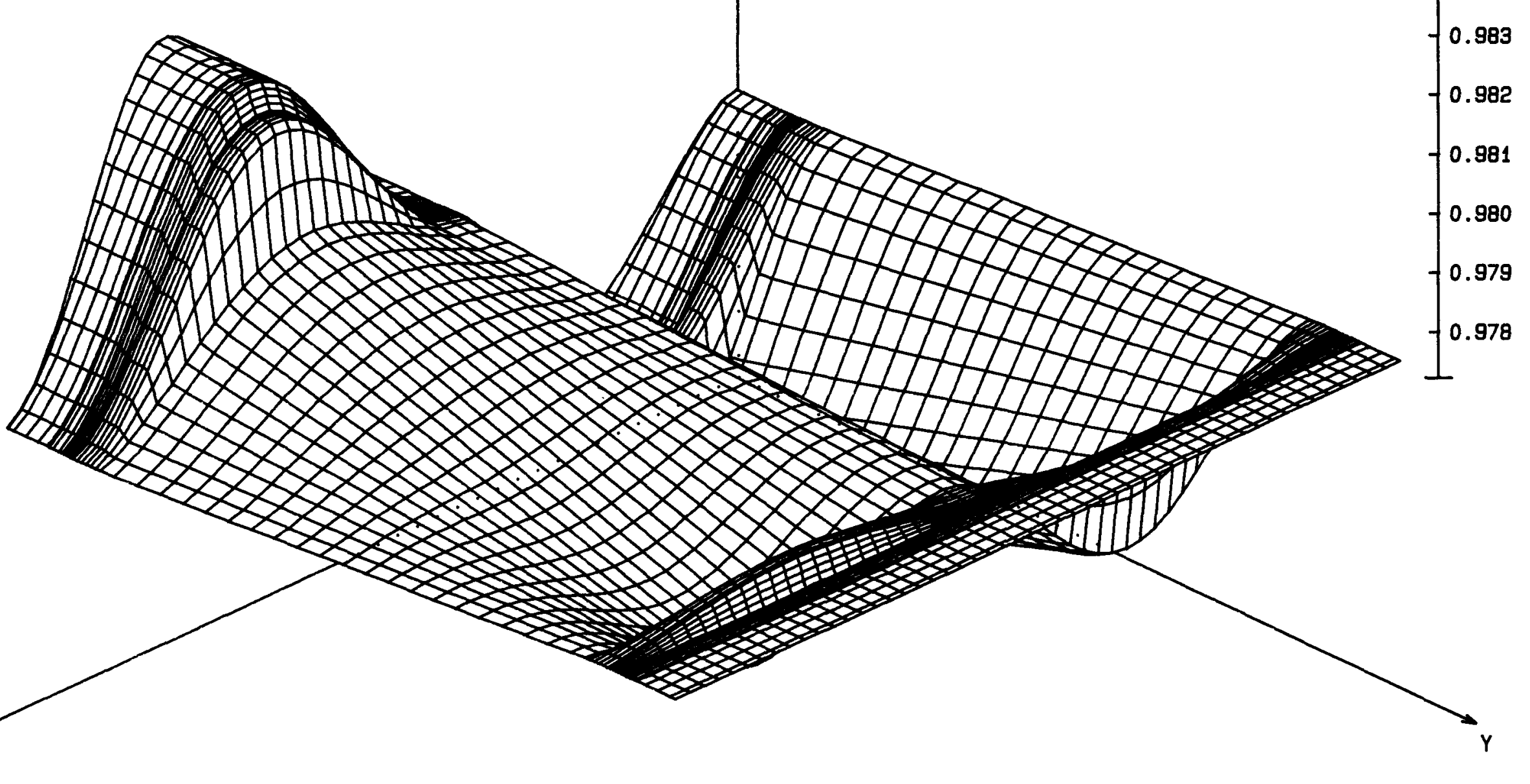

FIGURE 5.6 SYSTEM PRESSURE PROFILE FROM 1-D CODE: $11.3 \mathrm{rpm}$ 
$X$ AXIS: ANGLE (deg)

MIN: 0.000 MAX: 360.000

Y AXIS: POSITION $(\mathrm{cm})$

MIN: -36.148 MAX: 363.360

$Z$ AXIS: PRESSURE (bar)

MIN: 0.979 MAX: 0.984

$\overrightarrow{8}$

$\vec{\circ}$

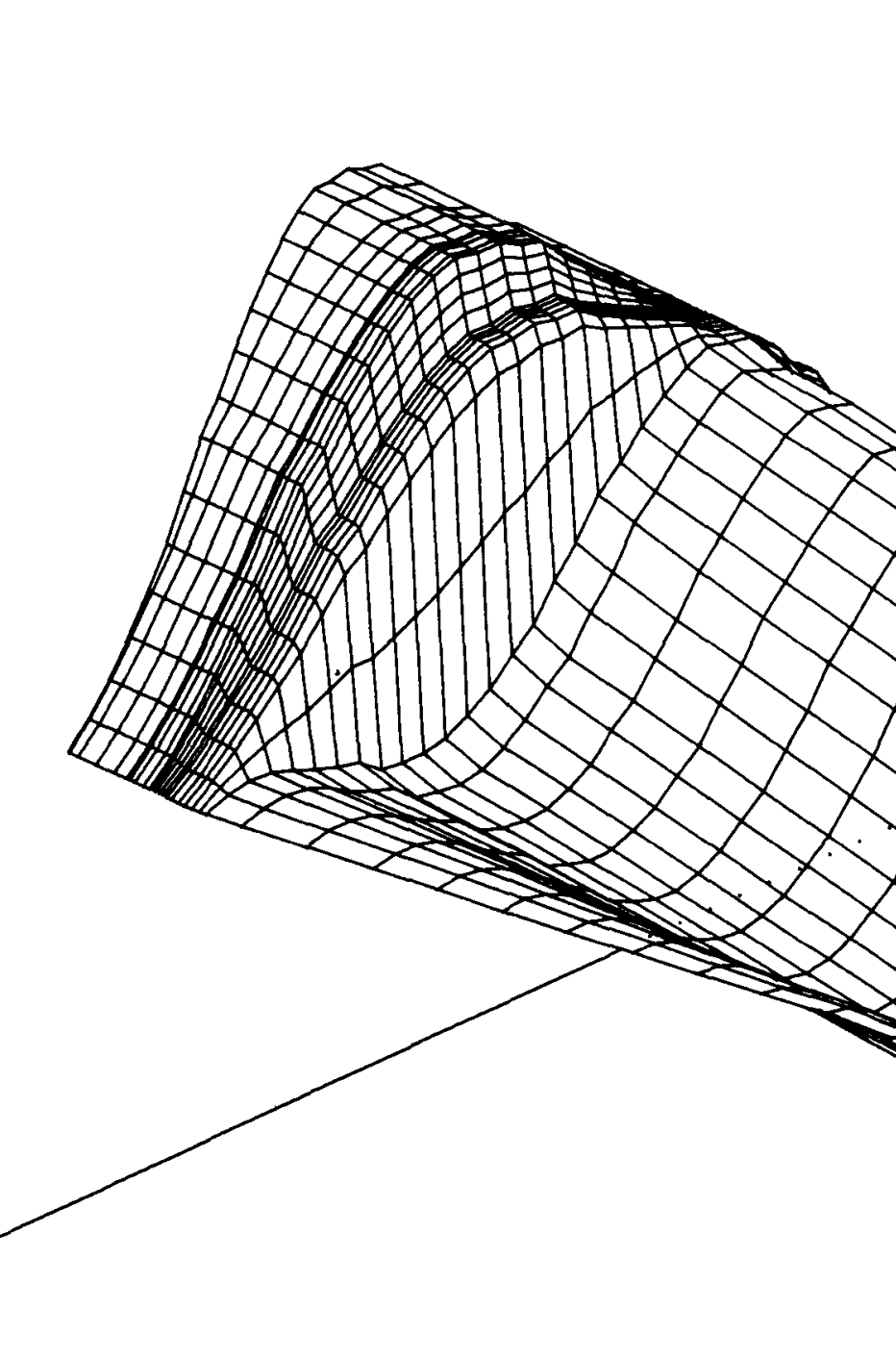

FIGURE 5.7 SYSTEM PRESSURE PROFILE FROM 2-D CODE: $11.3 \mathrm{rpm}$ 


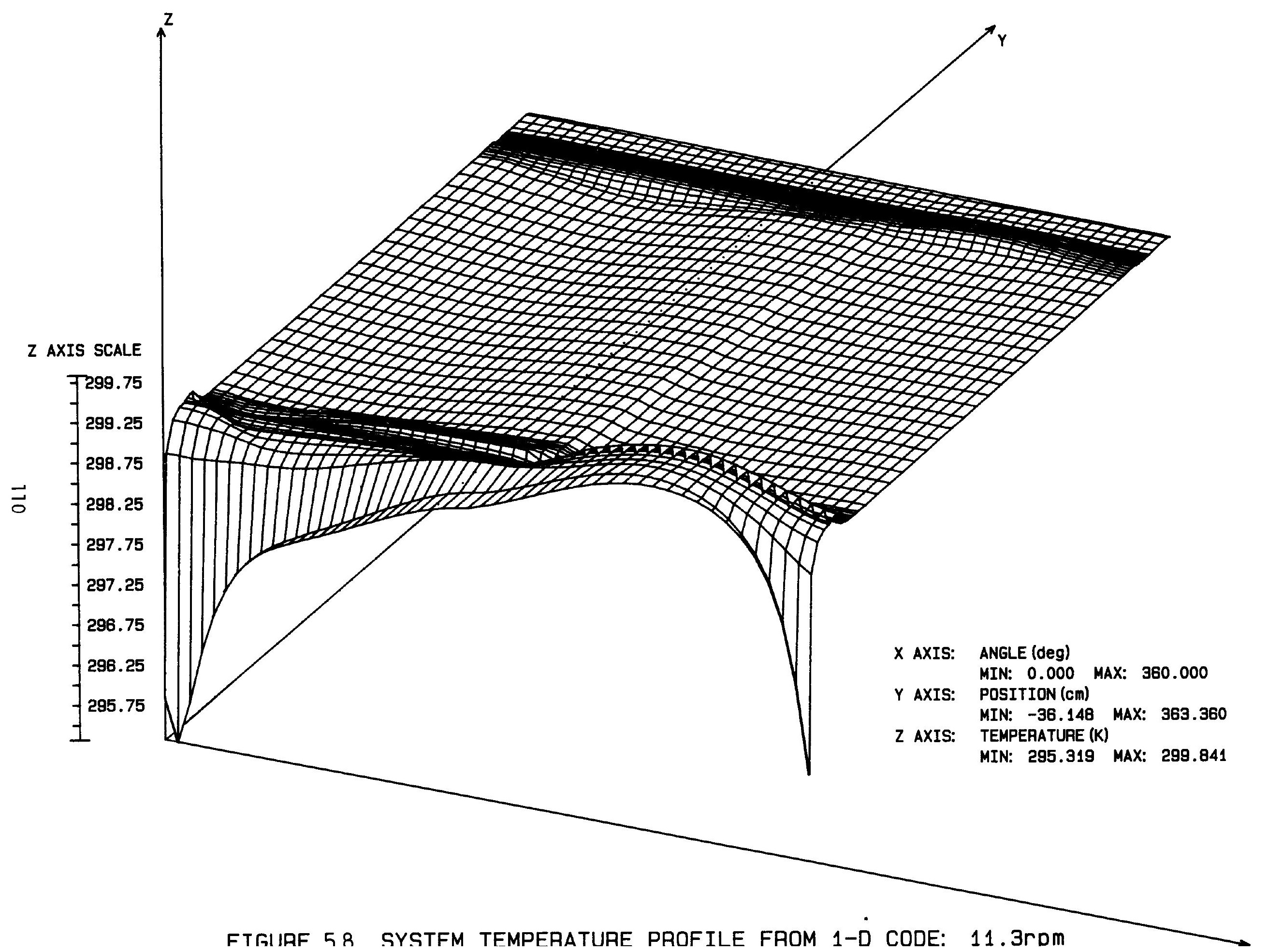




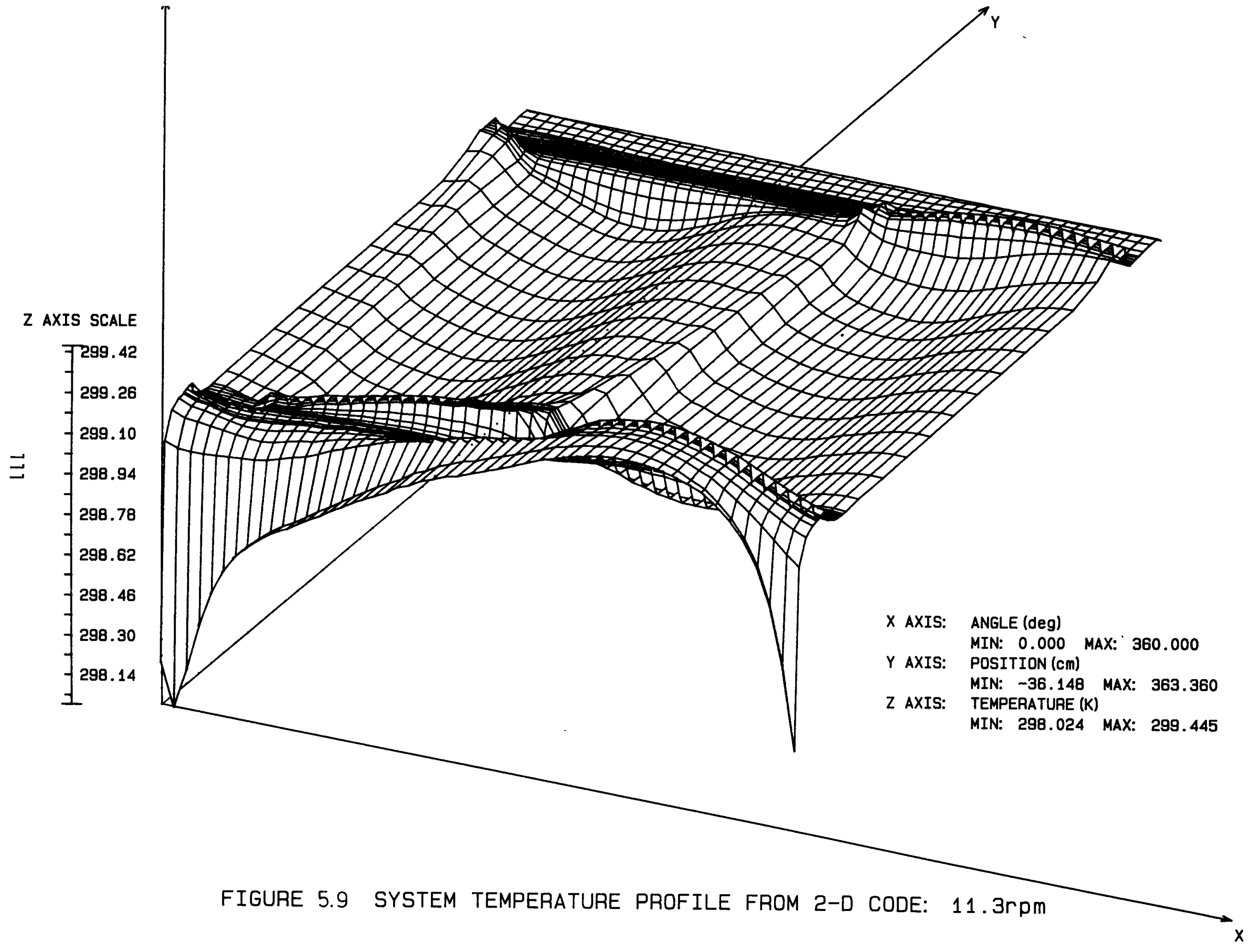


Conversely, during positive flow (between $180^{\circ}$ and $360^{\circ}$ ) the velocity at $609 \mathrm{~mm}$ should be less than that at $1304 \mathrm{~mm}$ (shown by the simulated data), which does not agree with the experimental observations. Furthermore, the magnitude of the experimental velocity drop is not intuitively credible in view of the small pressure drops expected in an atmospheric rig with piston leakage. After consultation with Seume, it was concluded that the most likely cause of the discrepancy is a systematic error in the hot-wire anemometer calibration. The 609 and $1304 \mathrm{~mm}$ tests were performed on different days and the anemometer calibration procedures used did not account for barometric pressure differences. Hence, because hot-wire anemometry is dependent on velocity as well as density, an ambient pressure variation of about 5 to 7 \& could account reasonably for the apparent axial velocity gradient measured. An additional source of systematic error is the manner in which the radial mean velocities measured are spatially integrated to yield the bulk flow velocity. It was thus agreed that future tests should include anemometer calibration procedures that include the effect of ambient pressure. Also, a better method of computing the bulk velocity was suggested.

A comparison of the system pressure profiles generated by the one- and two-dimensional codes at $11.3 \mathrm{rpm}$ is shown in figures 5.6 and 5.7. The pressure drop across the test section predicted by the one-dimensional code is larger than that predicted by the two-dimensional code over the cycle. Hence the pseudo-steady-state friction factor assumption used in the one-dimensional code generates larger dissipation than that produced by the two-dimensional code. The differences in profile topology apparent principally at the nozzle/test section interfaces are a manifestation of the difference in bulk fluid temperatures produced by the one- and two-dimensional codes. The twodimensional code produces a lower bulk temperature over the test section than its one-dimensional counterpart (see figures 5.8 and 5.9 ). This is intuitively reasonable since the one-dimensional code, which uses a pseudosteady-state heat transfer correlation, does not describe radial temperature gradients. Such gradients are shown clearly by the radial temperature profile (figure 5.10) produced by the two-dimensional code. Over most of the cycle, the temperatures adjacent to the wall are relatively larger than those of the flow core even though the net temperature difference of $.3 \mathrm{~K}$ is small. Thus, in the two-dimensional code, the actual wall/fluid heat transfer occurs in the boundary layer with the radial temperature gradient being constrained chiefly by turbulent enthalpy transport. Hence the extent to which the bulk temperatures predicted by the one- and two-dimensional codes differ is probably a direct effect of the exclusion of such transport from the twodimensional simulation.

Another difference between figures 5.6 and 5.7 is the relative smoothness of the one-dimensional plot. The jaggedness produced by the twodimensional code is caused by residual simulated turbulence below the cut-off of the numerical filter used to exclude such turbulence deliberately. It is interesting to note that the occurrence of the low level noise passing the filter is approximately in phase with the corresponding high levels of measured RMS velocity fluctuation shown in figures 5.11 and 5.12 . The magnitudes of the pressure drops simulated across the test section are less than $500 \mathrm{~Pa}$. An assessment of the accuracy of these predictions requires the 
$X$ AXIS: ANGLE (deg)

MIN: 0.000 MAX: 360.000

$Y$ AXIS: RADIUS (mm)

MIN: 1.684 MAX: 19.050

$Z$ AXIS: TEMPERATURE (K)

MIN: 298.937 MAX: 299.222

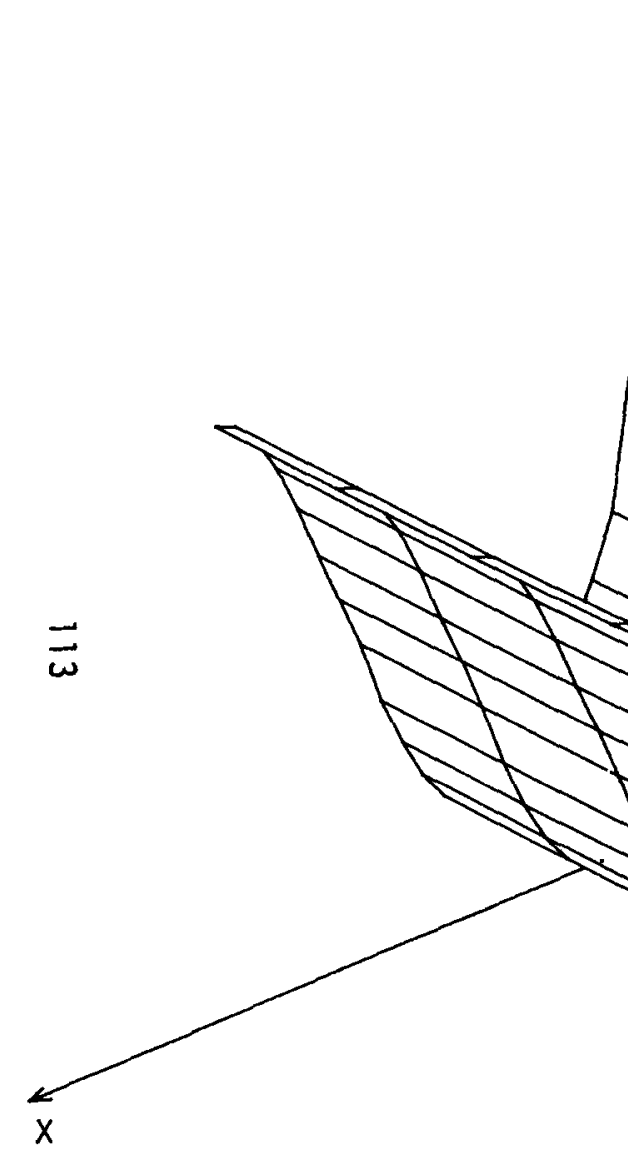

Z AXIS SCALE

T

299.20

$-299.16$

299.12

299.08

299.04

299.00

298.96

FIGURE 5.10 SIMUL. RADIAL TEMPERATURE PROFILE AT $1304 \mathrm{~mm}: 11.3 \mathrm{rpm}$ 


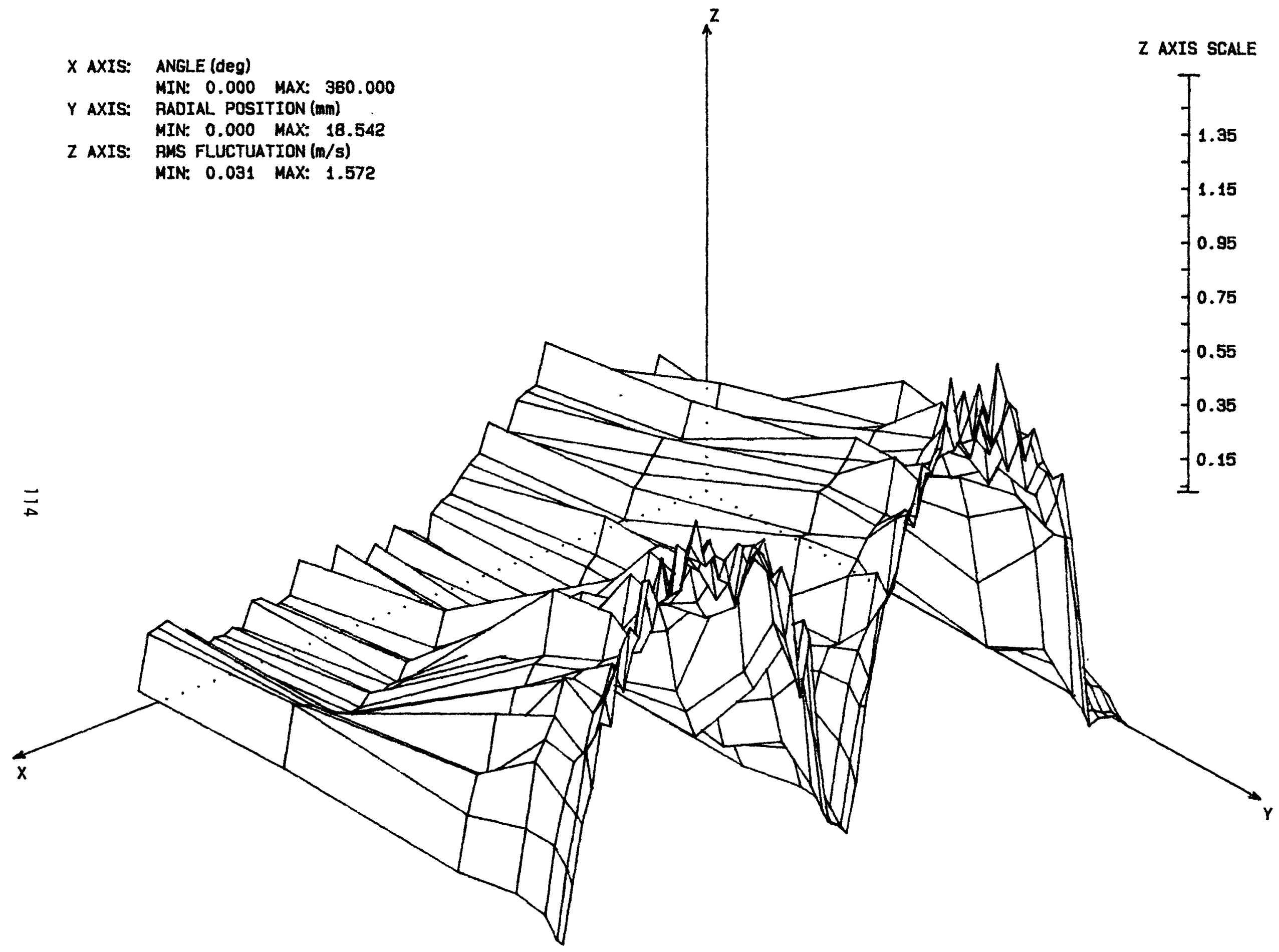

FTFIJRF 511 EXP. RADIAL PROFILE OF AXIAL RMS VEL. FLUCT. AT 609mm: 11.3rpm 


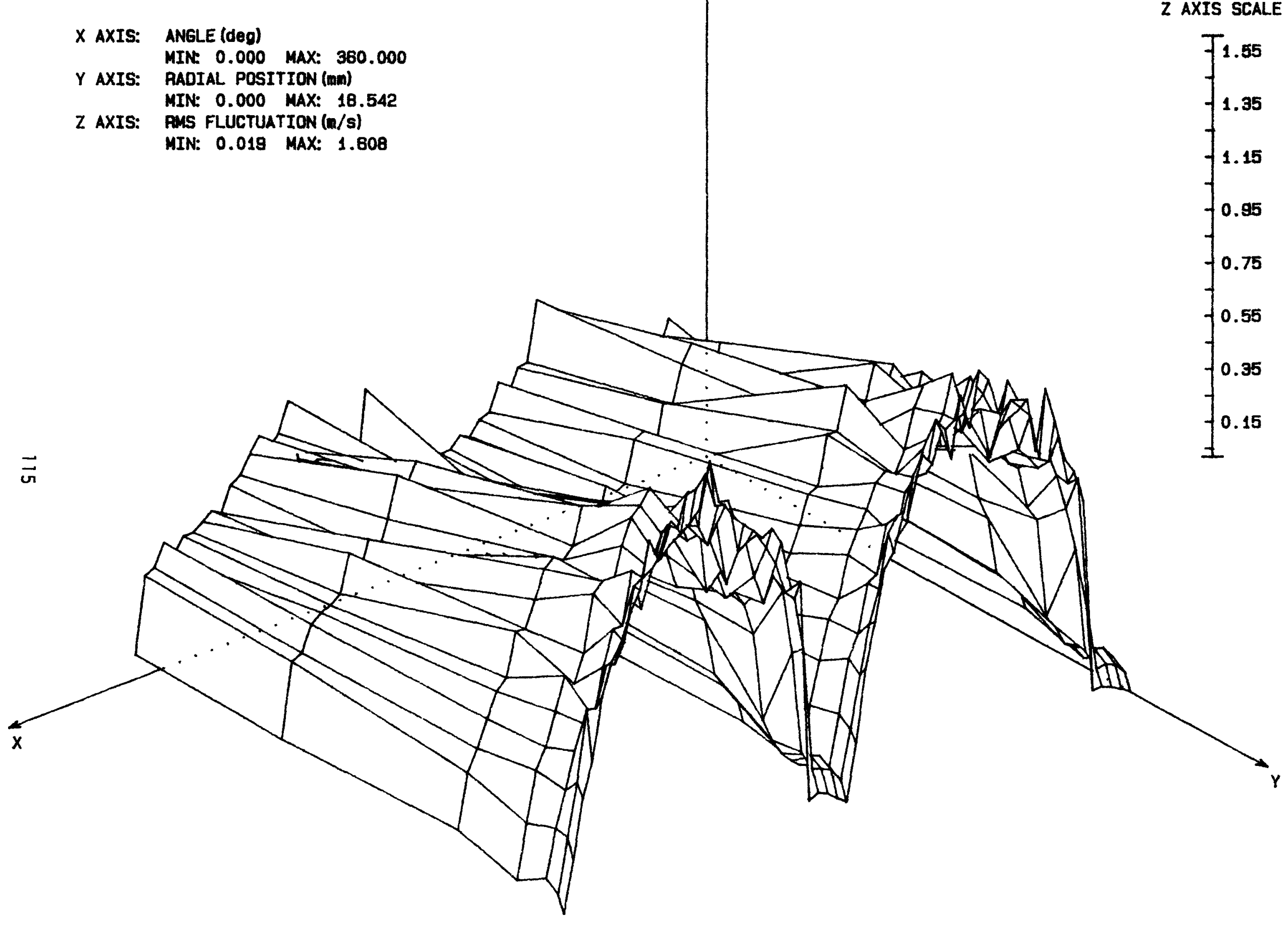

FIGURE 5.12 EXP. RADIAL PROFILE OF AXIAL RMS VEL. FLUCT. AT 1304mm: 11.3rpm 
installation of appropriate pressure transducers on the test rig. Hopefully, this will be accomplished in the future.

The system temperature profiles of figures 5.8 and 5.9 share a common topology but the two-dimensional profile magnifies the deviations from the isothermal mean over the test section. The adiabatic temperature variation in the cylinder is readily apparent, although the physical significance is exaggerated by scale effects ( 1 to $4 \mathrm{~K}$ in $300 \mathrm{~K}$ ).

The measured root-mean-square (RMS) velocity fluctuation profiles shown in figures 5.11 and 5.12 reveal the structure of the turbulence occurring in the test section. From figure 5.11, it may be observed that when the flow is negative, the centerline turbulence is considerably greater than that occurring when the flow is positive. This shows the extent to which turbulence is advected down the tube in the flow direction. In the positive flow direction, the $609 \mathrm{~mm}$ probe location is towards the tube entrance where the turbulence is nominally small by design. Hence the centerline fluctuation is smaller than that occurring during negative flow when the measurement location is toward the test section exit. Concomitantly, figure 5.12 shows that the centerline velocity fluctuation is largely independent of flow direction since the measurements in this case are made at the test section midpoint; the advected turbulence is thus the same regardless of the flow direction. It is also noteworthy that the largest fluctuations occur close to the boundary layer interface in all cases and, further, that the magnitude of these fluctuations is largely unaffected by flow direction (figure 5.10).

\subsubsection{Comparison of System Results at $33.5 \mathrm{rpm}$}

A comparison of the bulk velocity profiles at a rig speed of $33.5 \mathrm{rpm}$ is shown in figure 5.13. The piston leakage effects observed in this case are different from those observed at $11.3 \mathrm{rpm}$ (figure 5.2) since no net leakage is apparent; there may even be a net mass gain. Figures 5.16 and 5.17 reveal pressure variations about the ambient pressure of 0.98274 bars (as do figures 5.6 and 5.7 at $11.3 \mathrm{rpm})$. Hence, a possible mechanism for the absence of net cyclic leakage at $33.5 \mathrm{rpm}$ is that pressure differential driven leakage across the piston in one flow direction is canceled by mass gain when the flow reverses. However, this is not a totally satisfactory explanation in light of figures 5.6 and 5.7 (reversing pressure gradient with net leakage (figure 5.3)). A more probable hypothesis is that at $33.5 \mathrm{rpm}$, the period during which leakage flow exists is significantly smaller than that at $11.3 \mathrm{rpm}$. It may also be true that at higher speeds, the piston seals function better as a result of increased seal temperatures caused by greater friction. The net conclusion, therefore, is that piston leakage is an important boundary condition for the test section simulation and it requires more sophisticated experimental and modelling attention.

Ignoring the irregularity in the experimental bulk velocity profiles alluded to earlier (particularly at about $122^{\circ}$ ), the predicted and experimental bulk velocity profiles of figure 5.13 are generally in phase. The phase agreement of the one-dimensional profile is better than that of the two-dimensional profile, which may be ascribed to differences in dissipation 


\section{SIMULATED AND EXPERIMENTAL BULK VELOCITY PROFILES}

RIG SPEED: $33.5 \mathrm{rpm}$ PROBE POSITION: $1304 \mathrm{~mm}$

$1-d$ simul.

2-d simul.

experıment

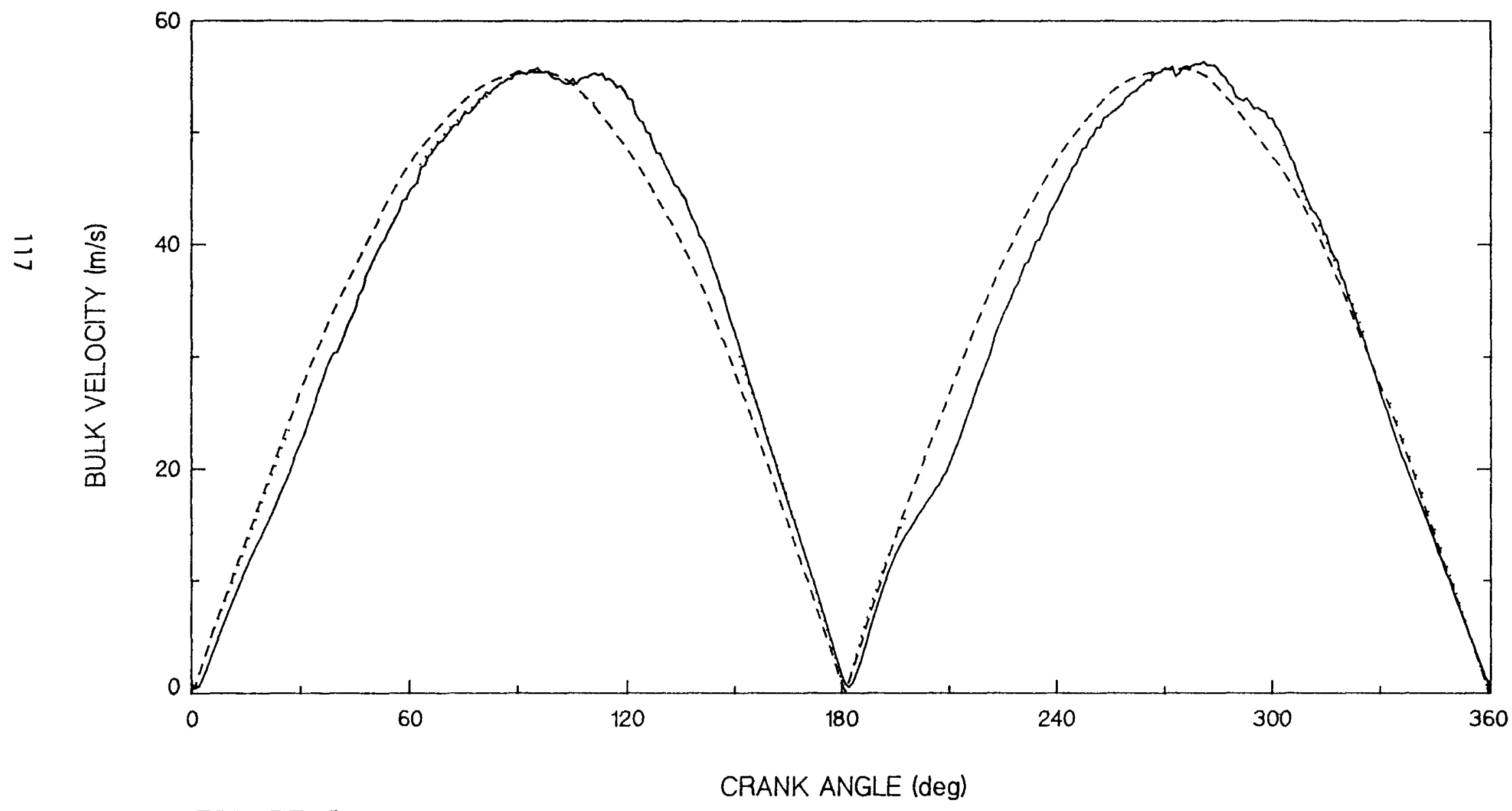

FIGURE 513 
$X$ AXIS: ANGLE (deg)

MIN: 0.000 MAX: 360.000

$Y$ AXIS:

POSITION (cm)

MIN: -36.148 MAX: 363.360

$Z$ AXIS: VELOCITY $(\mathrm{m} / \mathrm{s})$

MIN: -55.509 MAX: 55.818
Z AXIS SCALE

T50.0

40.0

30.0

20.0

10.0

0.0

$-10.0$

$-20.0$

$-30.0$

$-40.0$

$-50.0$

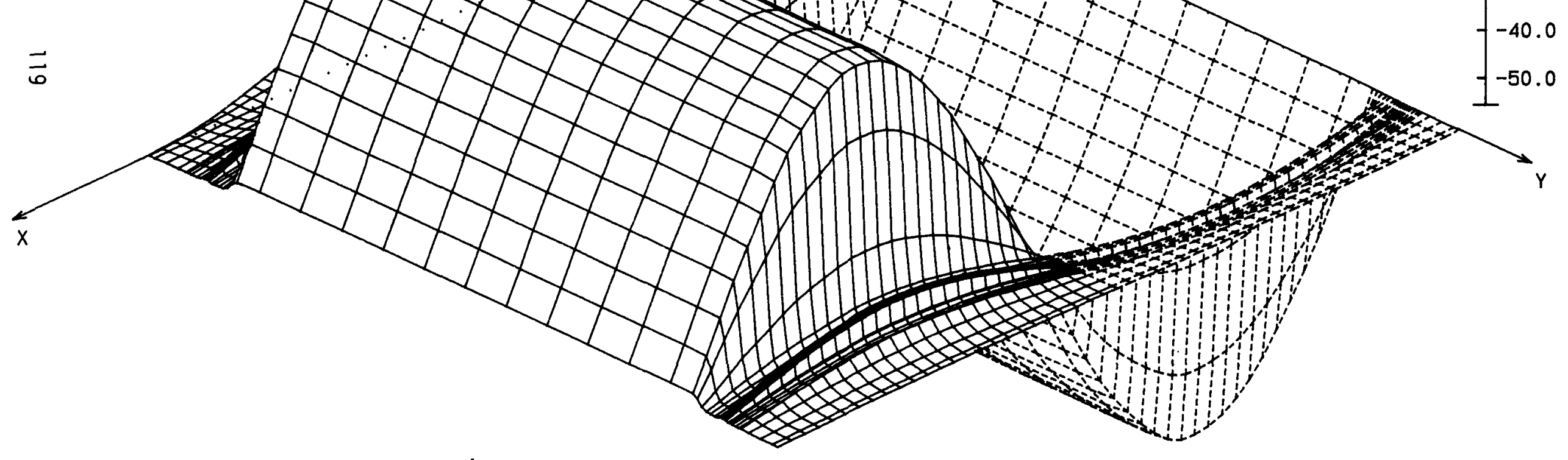

FIGURE 5.15 SYSTEM AXIAL VELOCITY PROFILE FROM 2-D CODE: $33.5 \mathrm{rpm}$ 


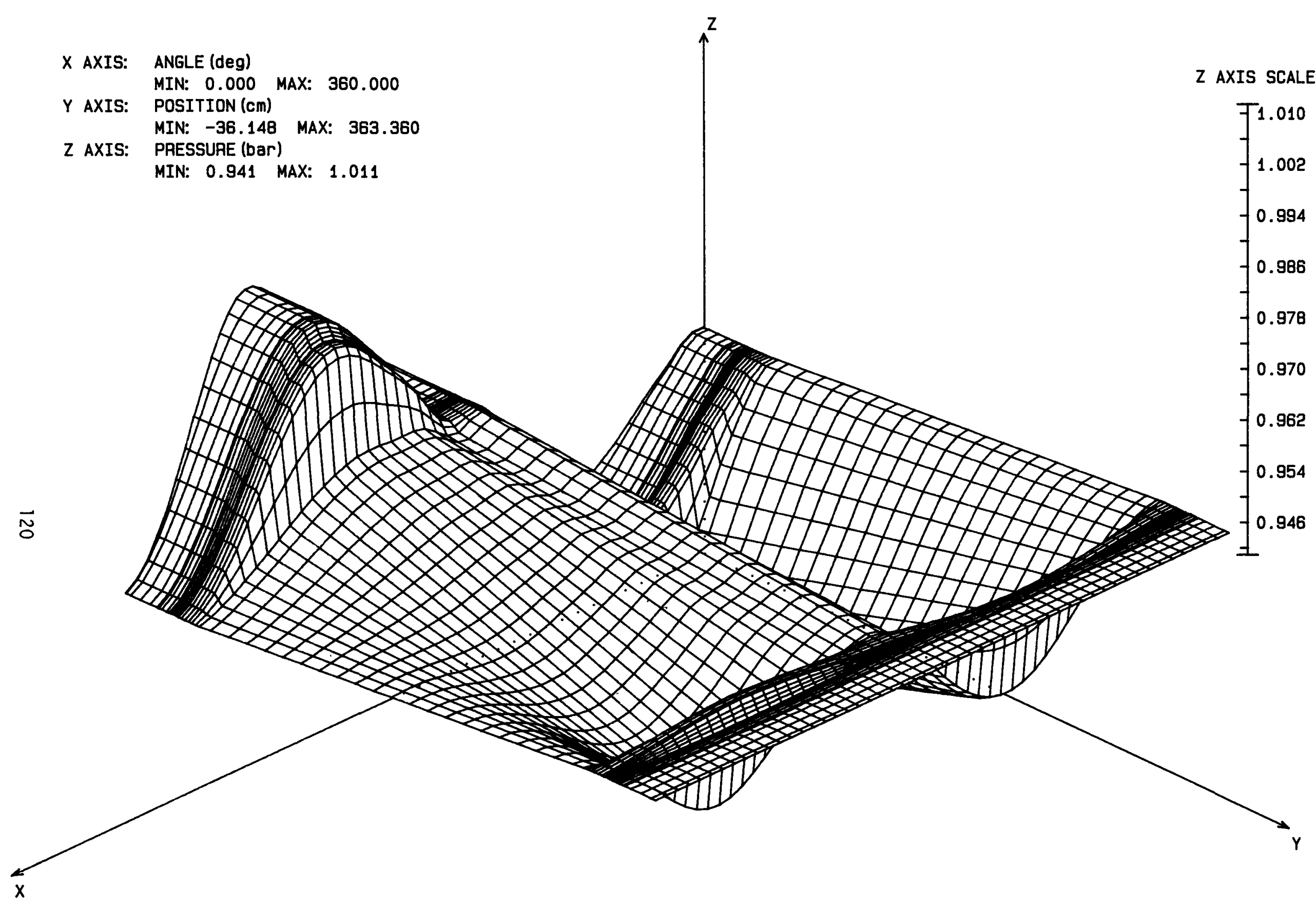

FIGURE 5.16 SYSTEM PRESSURE PROFILE FROM 1-D CODE: $33.5 \mathrm{rpm}$ 


\section{$X$ AXIS: ANGLE (deg)}

MIN: 0.000 MAX: 360.000

POSITION (cm)

MIN: -36.148 MAX: 363.360

$Z$ AXIS: PRESSURE (bar)
MIN: 0.958 MAX: 0.993

$Z$ AXIS: PRESSURE (bar)
MIN: 0.958 MAX: 0.993

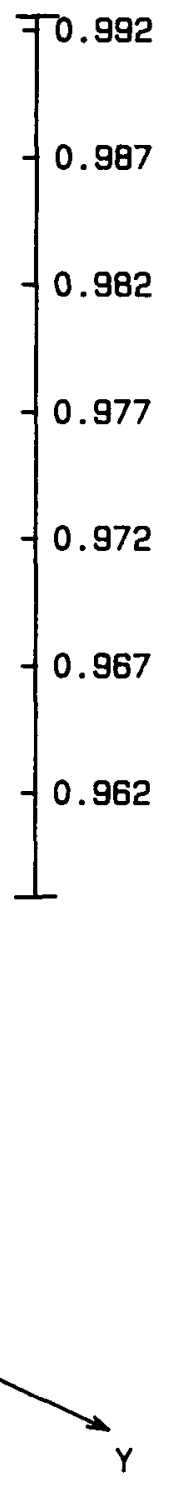

$\vec{\simeq}$

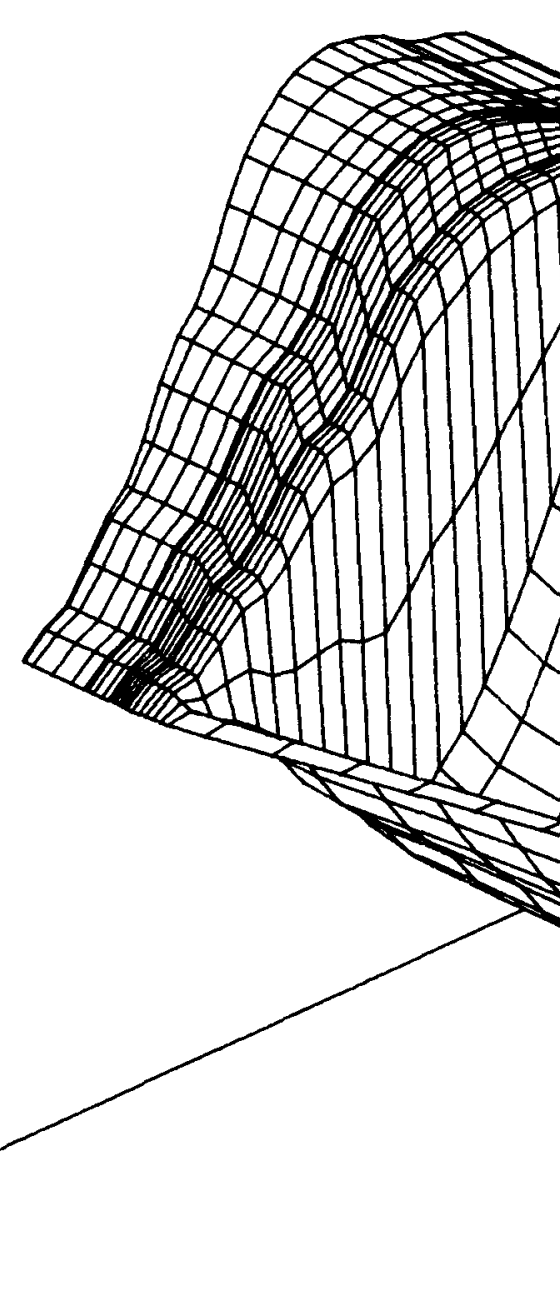

FIGURE 5.17 SYSTEM PRESSURE PROFILE FROM 2-D CODE: $33.5 \mathrm{rpm}$ 
modelling. As noted above, the pseudo-steady-state friction factor based calculation method used in the one-dimensional code produces more dissipation than the two-dimensional code. The reduction in simulated dissipation at 33.5 rpm (peak Reynolds numbers greater than 131,000) caused by neglecting turbulence in the two-dimensional model is thus manifest on a system basis, a manifestation absent at $11.3 \mathrm{rpm}$ (see figures 5.2 and 5.3 ).

The system axial velocity profiles depicted in figures 5.14 and 5.15 are identical in shape; the reduction in axial discretisation is apparent in the profile generated by the two-dimensional code. Comparing these figures with those generated at $11.3 \mathrm{rpm}$ (figures 5.4 and 5.5 ), it is evident that the system axial velocity profile topology is independent of rig speed, which principally affects the peak flow velocity. This is consistent for an openended system operating at atmospheric conditions.

The system pressure profiles produced by the one- and two-dimensional codes at $33.5 \mathrm{rpm}$ shown in figures 5.16 and 5.17 are similar in shape to their counterparts at $11.3 \mathrm{rpm}$ given by figures 5.6 and 5.7 , respectively. The features of these latter figures are accentuated at $33.5 \mathrm{rpm}$, particularly in the two-dimensional case (figure 5.17). The impact of lower bulk mean fluid temperatures simulated by the two-dimensional code in the test section is readily apparent. Figure 5.20 reveals a radial temperature difference between the wall and the centerline exceeding $2^{\circ}$ compared with $.3^{\circ}$ at $11.3 \mathrm{rpm}$. This is prima facie evidence of the importance of explicitly including turbulence phenomena in the simulation as well as the garnering of sufficient experimental heat transfer data to investigate the validity of the twodimensional code thermal predictions. The jaggedness of figure 5.17 compared with its one-dimensional counterpart in figure 5.16 is notable since the jaggedness is more pronounced than that occurring at $11.3 \mathrm{rpm}$ (figure 5.7). The observed pressure perturbations are a direct result of the temperature striations shown in figures 5.18 and 5.19 (which again are significantly greater than at $11.3 \mathrm{rpm}$ as reflected by figures 5.8 and 5.9 ). This is largely ascribable to the increased turbulence level, which produces peak RMS velocity fluctuations of $5.6 \mathrm{~m} / \mathrm{s}$ at $33.5 \mathrm{rpm}$ (figure 5.21 ) compared with only $1.6 \mathrm{~m} / \mathrm{s}$ at $11.3 \mathrm{rpm}$.

Ignoring the unfiltered turbulence effects, further comparison of figures 5.18 and 5.19 shows that the basic temperature profile of the rig at $33.5 \mathrm{rpm}$ is more complex and less isothermal than at $11.3 \mathrm{rpm}$. A comparison of figures 5.8 and 5.18 (one-dimensional predictions) shows that the flow is not nearly as isothermal over the test section at $33.5 \mathrm{rpm}$ as it is at 11.3 rpm. Of particular note is the relatively large drop in temperature between the heat exchangers and the test section. Part of this temperature gradient occurs as a result of the flow acceleration/deceleration in the nozzles where the reversible thermal/mechanical energy transfer (vdp) is greatest (see figures 5.16 and 5.17). This emphasizes the importance of generating accurate boundary condition data for any two-dimensional simulation of the test section. It also suggests that the rig instrumentation should be upgraded to include as complete a definition of transient test section boundary conditions 


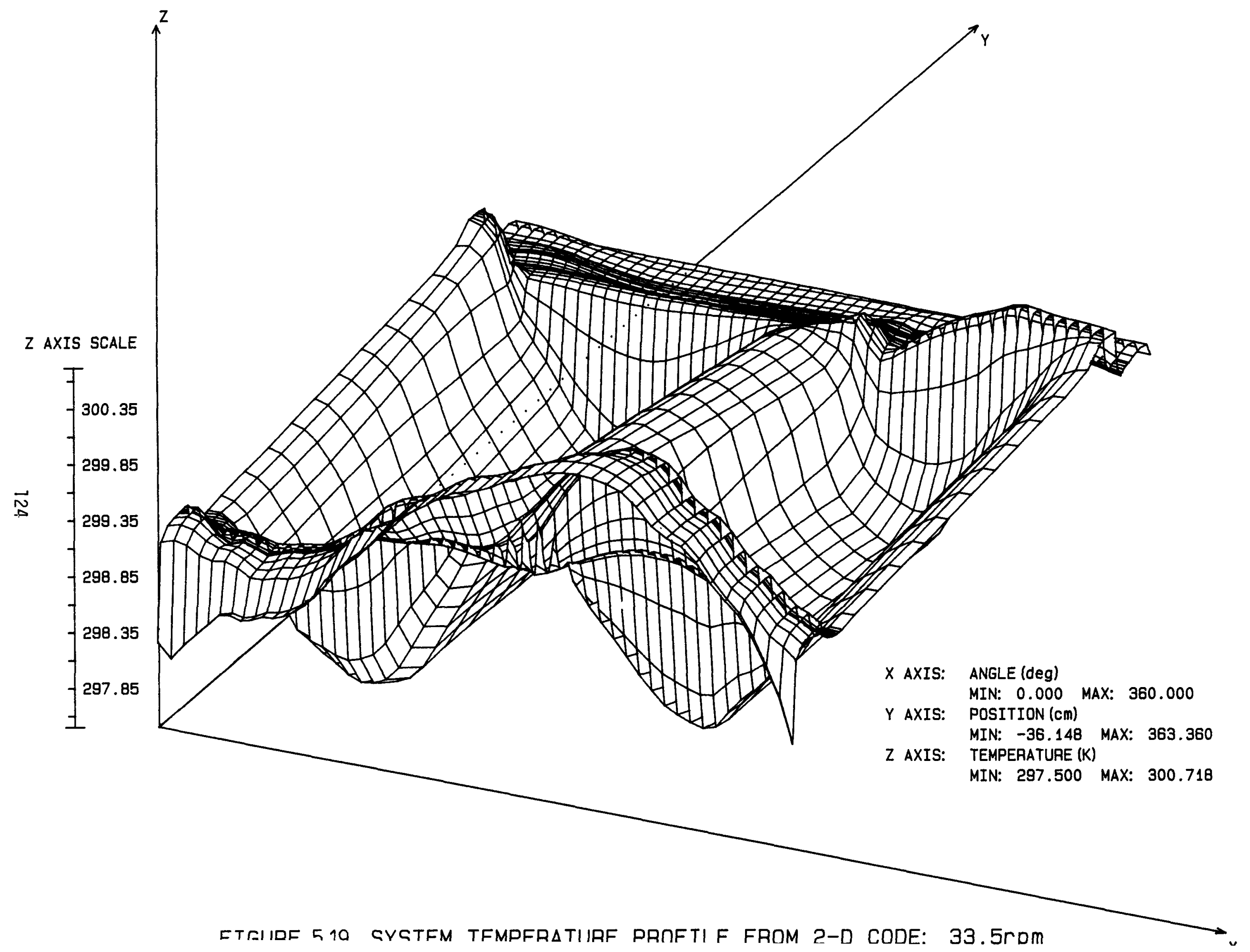




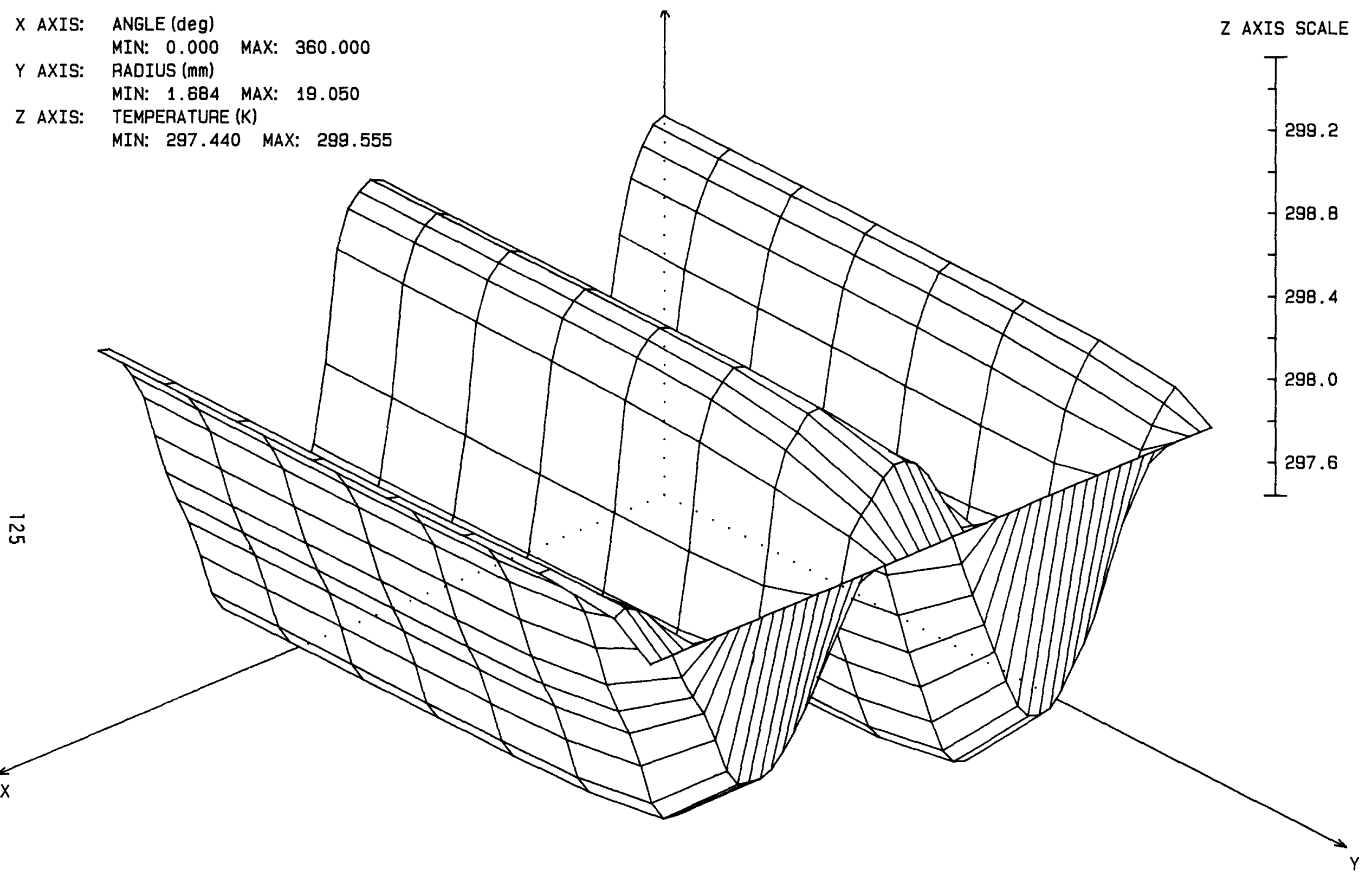

FIGURE 5.20 SIMUL. RADIAL TEMPERATURE PROFILE AT $1304 \mathrm{~mm}: 33.5 \mathrm{rpm}$ 
as possible. This is extremely important when attempting to apply the rig test data to actual Stirling hardware, which generally has very different heater and cooler tube boundary conditions than those of the rig.

Furthermore, it is important to ascertain the influence of boundary conditions on the test data since, particularly at elevated rig speeds, boundary condition effects may overwhelm the physical similarity between the rig and Stirling machine heat exchangers.

At $33.5 \mathrm{rpm}$, the radial temperature profile of figure 5.20 is comparable in shape to that at $11.3 \mathrm{rpm}$ (figure 5.10). Other than the radial temperature difference noted above, careful inspection shows figure 5.10 (at $11.3 \mathrm{rpm}$ ) to have irregularities at the points of flow reversal $\left(0^{\circ}\right.$ and $180^{\circ}$, figure 5.5) which are absent at $33.5 \mathrm{rpm}$. The reasons for this are not clear and require further investigation.

Many of the features of the RMS velocity fluctuation profile measured at $11.3 \mathrm{rpm}$ (figure 5.12) are evident at $33.5 \mathrm{rpm}$ in figure 5.21. The centerline fluctuations appear to be relatively smaller in magnitude at $33.5 \mathrm{rpm}$ while the degree of irregularity toward the tube wall apparently is not much affected by the rig speed. The monotonic relationship between $\mathrm{rig}$ speed and peak RMS fluctuation is also evident; a threefold increase in rig speed produces a peak RMS velocity fluctuation ratio of 3.5. This may prove useful in devising better friction factor and heat transfer factor correlations for use in one-dimensional simulations.

\subsubsection{Comparison of Test Section Results at $11.3 \mathrm{rpm}$}

Cyclic perspectives on the simulated and measured radial profiles of mean axial velocity at $11.3 \mathrm{rpm}$ and at the center of the test section (probe position of $1304 \mathrm{~mm}$ ) are given in figures 5.22 and 5.23 . The absence of negative velocities in figure 5.22 is a result of hot wire anemometry yielding data that have no flow direction discrimination. Clearly the simulated and experimental profiles have different shapes, with the experimental profile being more rounded. As noted for figures 5.2 and 5.3, the peak velocities simulated and measured are not dissimilar, their difference being related to piston leakage effects. Mechanically induced flow irregularities are also apparent in figure 5.22 (as discussed previously). Of particular note in figure 5.23 is the slight occurrence of positive and negative flows (the " $S$ " shape velocity profile) existing at the flow reversal points of 0 and $180^{\circ}$ (visible at $0^{\circ}$ and $360^{\circ}$ ). Bearing in mind the absolute nature of the experimental data, the same effect is discernable in figure 5.22 at $360^{\circ}$ where the absolute value of the velocity is greater toward the tube wall than at the centerline, which is not usually the case.

A better comparison of the shape of the radial velocity profiles is depicted 1 . figures 5.24 and 5.25 which are superimposed in figure 5.26. At $210^{\circ}$ to $275^{\circ}$, the simulated profiles are much flatter than their experimental counterparts although their terminating values at the tube centerline and at about $18.5 \mathrm{~mm}$ are comparable. At $200^{\circ}$, both experimental and simulated profiles have a negative radial gradient in the core flow region although the simulated gradient negativity is more pronounced. 


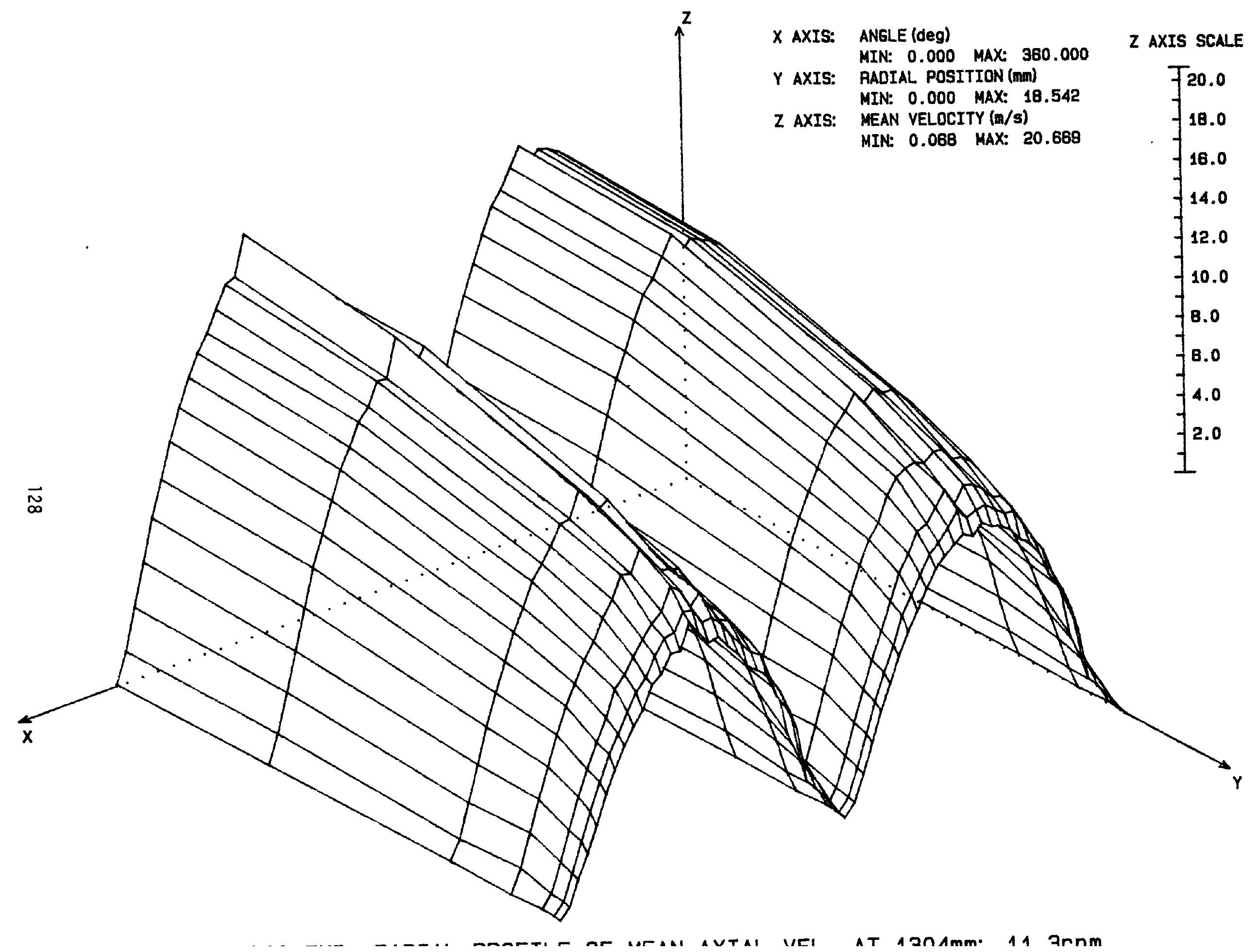




\section{SIMULATED RADIAL PROFILES OF MEAN AXIAL VELOCITY}

RIG SPEED: $11.3 \mathrm{rpm}$ PROBE POSITION: $1304 \mathrm{~mm}$

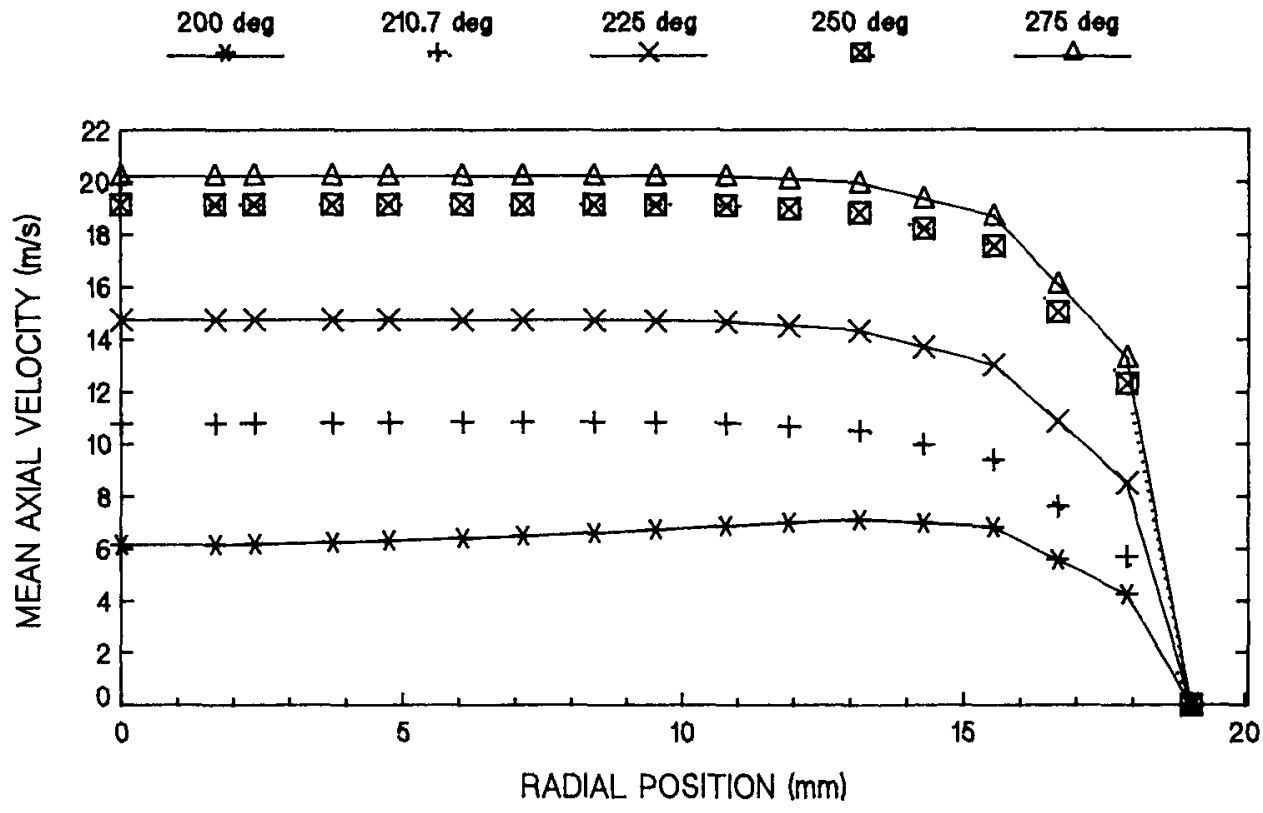

Figure 5.24

EXPERIMENTAL RADIAL PROFILES OF MEAN AXIAL VELOCITY RIG SPEED: $11.3 \mathrm{rpm}$ PROBE POSITION: $1304 \mathrm{~mm}$

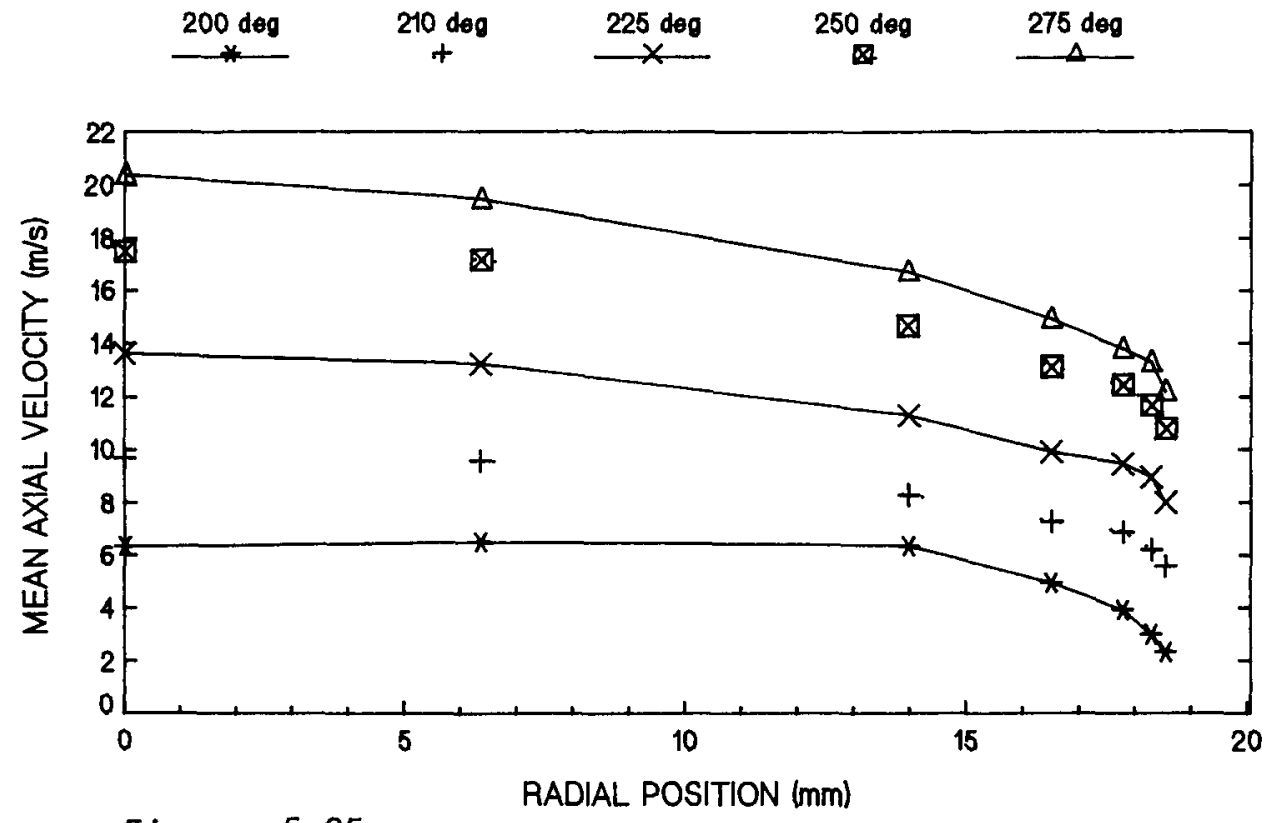

Figure 5.25 


\section{COMBINED RADIAL PROFILES OF MEAN AXIAL VELOCITY}

RIG SPEED: $11.3 \mathrm{rpm}$ PROBE POSITION: $1304 \mathrm{~mm}$

experiment

simulation

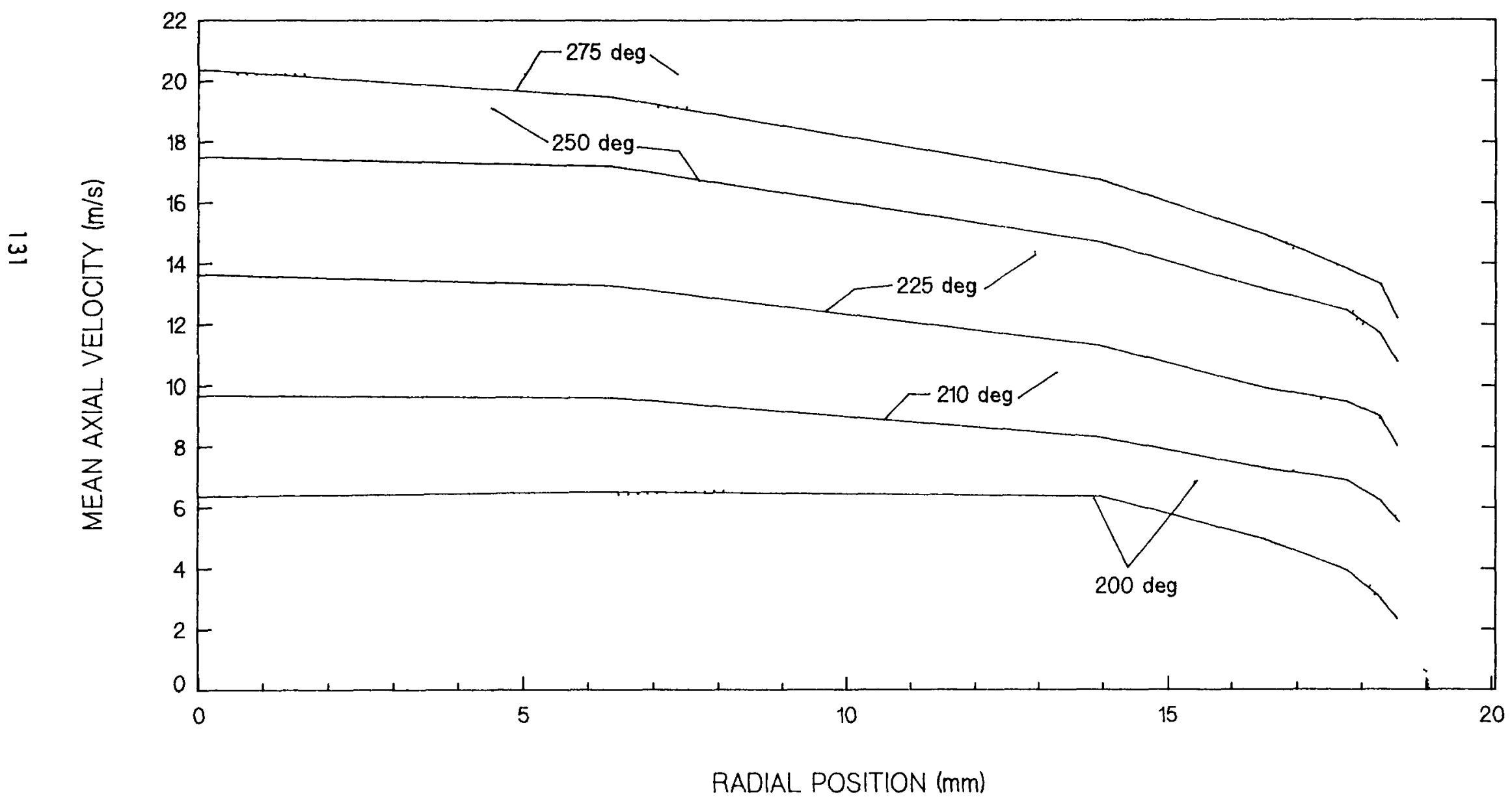

FIGURE 526 
An alternate explanation for the disagreement between the shape of the simulated and measured profiles could be sought in a mismatch of experimental and simulated test section boundary conditions. In other words, if the experimental flow at the test section / nozzle interface contains an embedded radial gradient of axial velocity, then the roundness of the experimental profiles could be a distorted version of the embedded boundary gradient. The absence of an embedded boundary gradient in the simulation (slab flow conditions are assumed at the test section entrance) would account for the observed discrepancy. However, figures 5.29 and 5.30 do not substantiate this hypothesis since the velocity profiles at a probe position of $609 \mathrm{~mm}$ from the entrance are much flatter than those at the test section midpoint. This behavior is consistent with the existence of slab flow at the test section entrance under experimental conditions. Thus it appears necessary to seek an explanation for the observed discrepancies in terms of other factors such as the exclusion of turbulence from the simulation.

\section{4 .5 Comparison of Test Section Results at $33.5 \mathrm{rpm}$}

The difference in shape noted for figures 5.22 and 5.23 at $11.3 \mathrm{rpm}$ is more pronounced at $33.5 \mathrm{rpm}$ as shown by figures 5.31 and 5.32 . In this case, the roundness of the experimental radial profile of mean axial velocity (figure 5.31) is sharply contrasted against figure 5.32, whose flatness at $33.5 \mathrm{rpm}$ is more marked than that of figure 5.23 at $11.3 \mathrm{rpm}$, particularly at the boundary layer interface. The reversed boundary flow (relative to the direction of the flow core) at 0 and $360^{\circ}$ is less than that revealed by figures 5.22 and 5.23; indeed, its existence in figure 5.31 is arguable.

The shapes of the radial profiles at discrete crank angles are shown separately for the simulated and experimental data in figures 5.33 and 5.34 respectively and together in figure 5.35. The reduction in roundness of the simulated profiles at $33.5 \mathrm{rpm}$ compared with those at $11.3 \mathrm{rpm}$ (figure 5.24) is evident. Figure 5.35 shows that the turbulence-induced radial flow effects are more apparent at $33.5 \mathrm{rpm}$ than at $11.3 \mathrm{rpm}$. This is consistent with an increase in peak measured RMS fluctuation from $1.6 \mathrm{~m} / \mathrm{s}$ at $11.3 \mathrm{rpm}$ to $5.6 \mathrm{~m} / \mathrm{s}$ at $33.5 \mathrm{rpm}$. The simulated peak radial flow velocity shown in figure 5.36 is still small at $-0.011 \mathrm{~m} / \mathrm{s}$; two orders of magnitude smaller than the turbulence fluctuations. Thus momentum conservation dictates that including higher negative radial velocities in the simulation would coerce the concurrence of figures 5.33 and 5.34. In this regard, it may also be noted that the volumetric flow rates produced by integrating the experimental and simulated axial velocity profiles with respect to radius would be similar (allowing for 


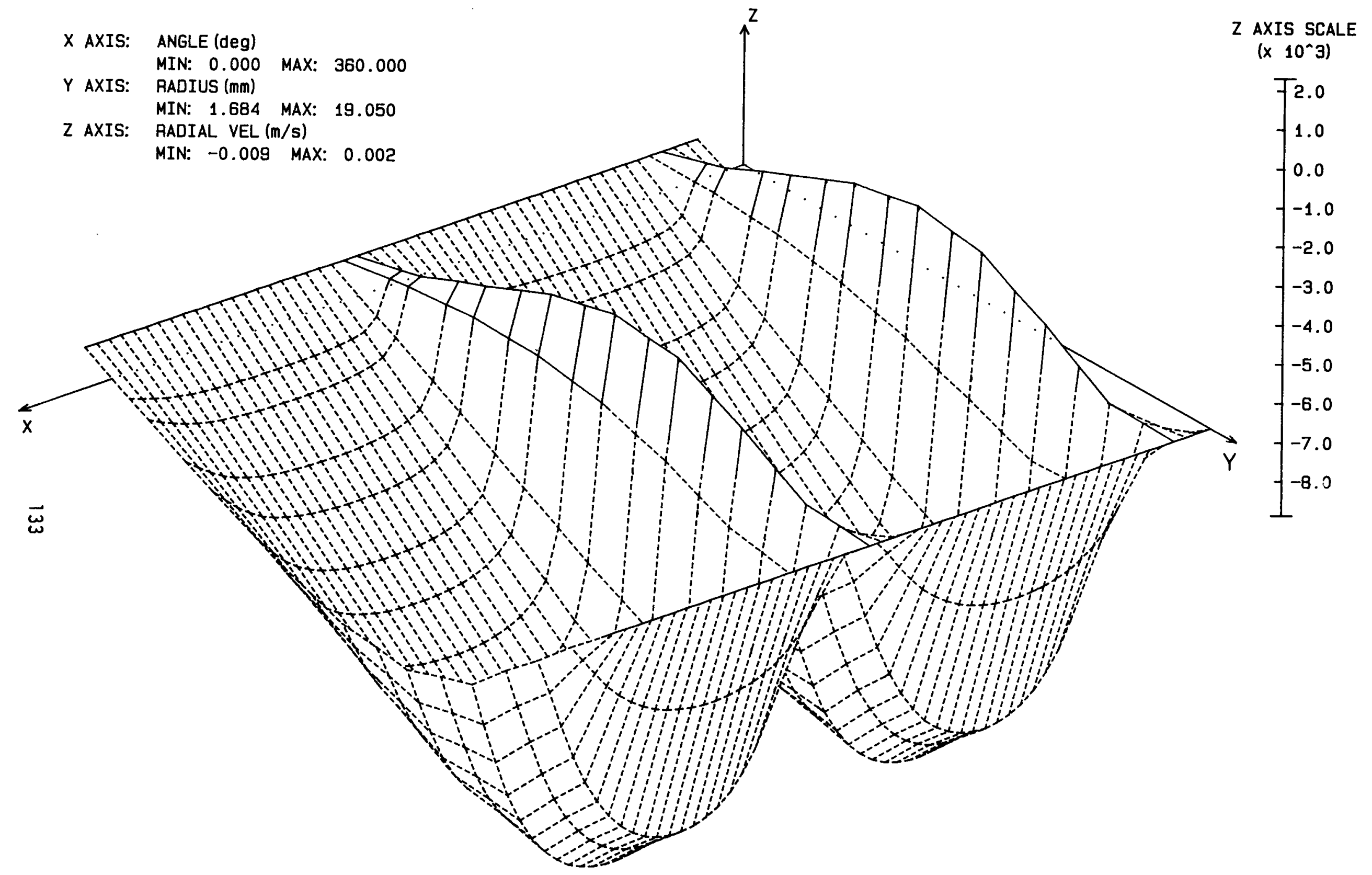

FIGURE 5.27 SIMUL. RADIAL PROFILE OF MEAN RADIAL VEL. AT 1304mm: 11.3rpm 


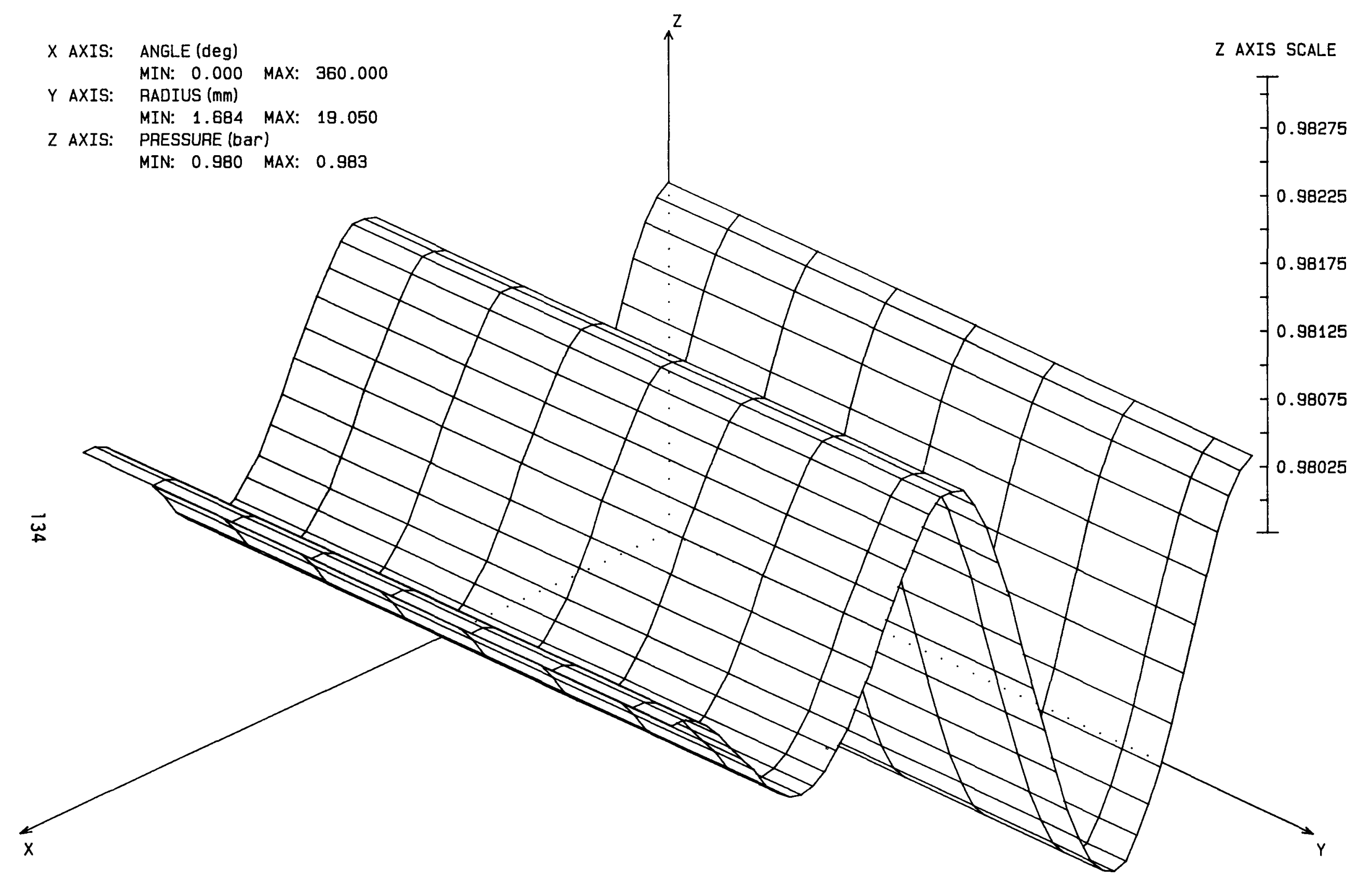

FIGURE 5.28 SIMUL. RADIAL PRESSURE PROFILE AT $1304 \mathrm{~mm}: 11.3 \mathrm{rpm}$ 


\section{$X$ AXIS: ANGLE (deg)}

MIN: 0.000 MAX: 360.000

$Y$ AXIS: RADIAL POSITION (mm)

MIN: 0.000 MAX: 18.542

$Z$ AXIS: MEAN VELOCITY $(m / s)$

MIN: 0.075 MAX: 20.631

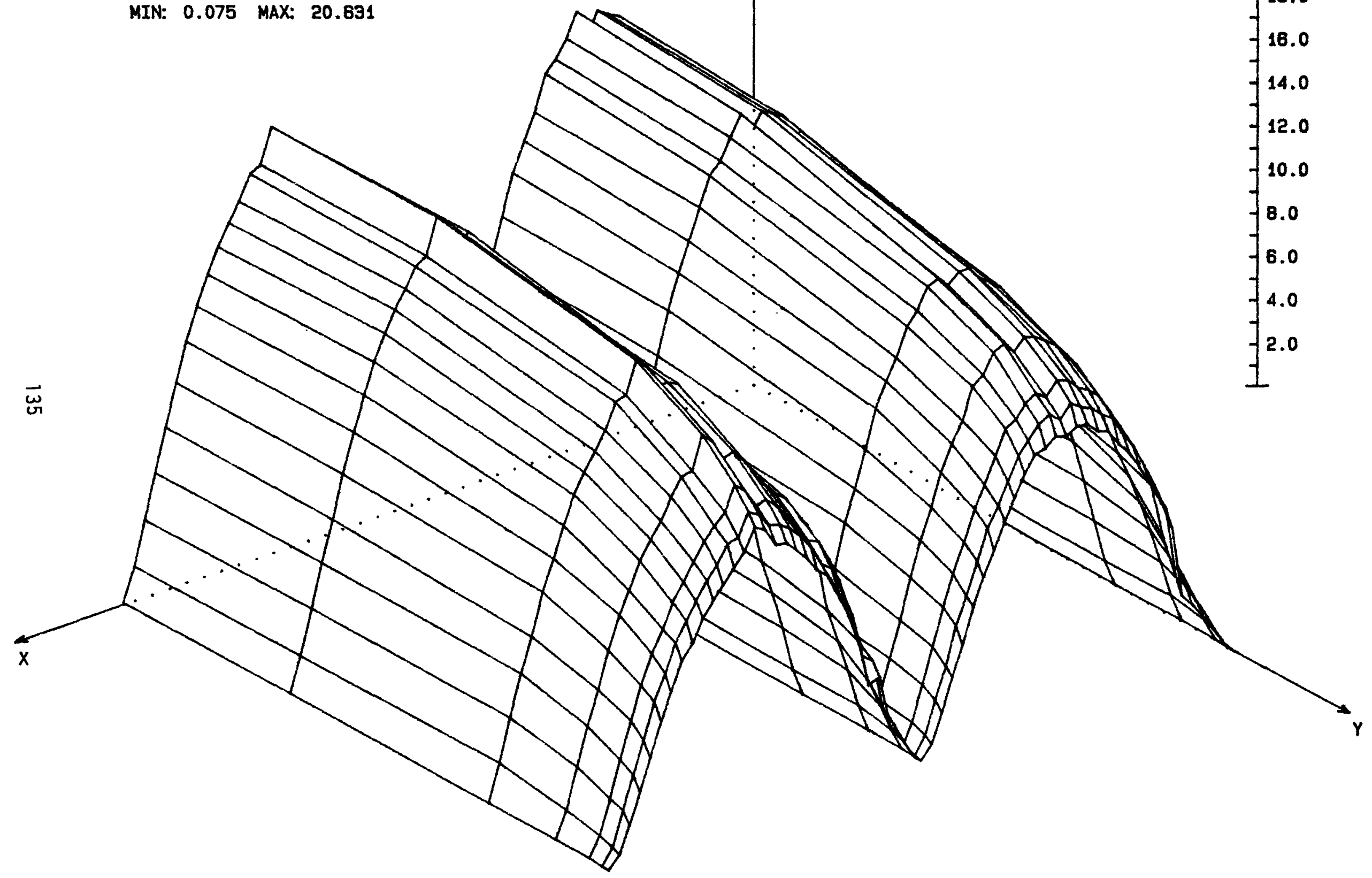

FIGURE 5.29 EXP. RADIAL PROFILE OF MEAN AXIAL VEL. AT 609mm: 11.3rpm 


\section{EXPERIMENTAL RADIAL PROFILES OF MEAN AXIAL VELOCITY}

RIG SPEED: $11.3 \mathrm{rpm}$ PROBE POSITION: 609mm

$200 \mathrm{deg}$
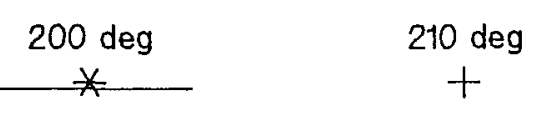

10 deg
+

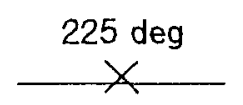

$250 \mathrm{deg}$

邓
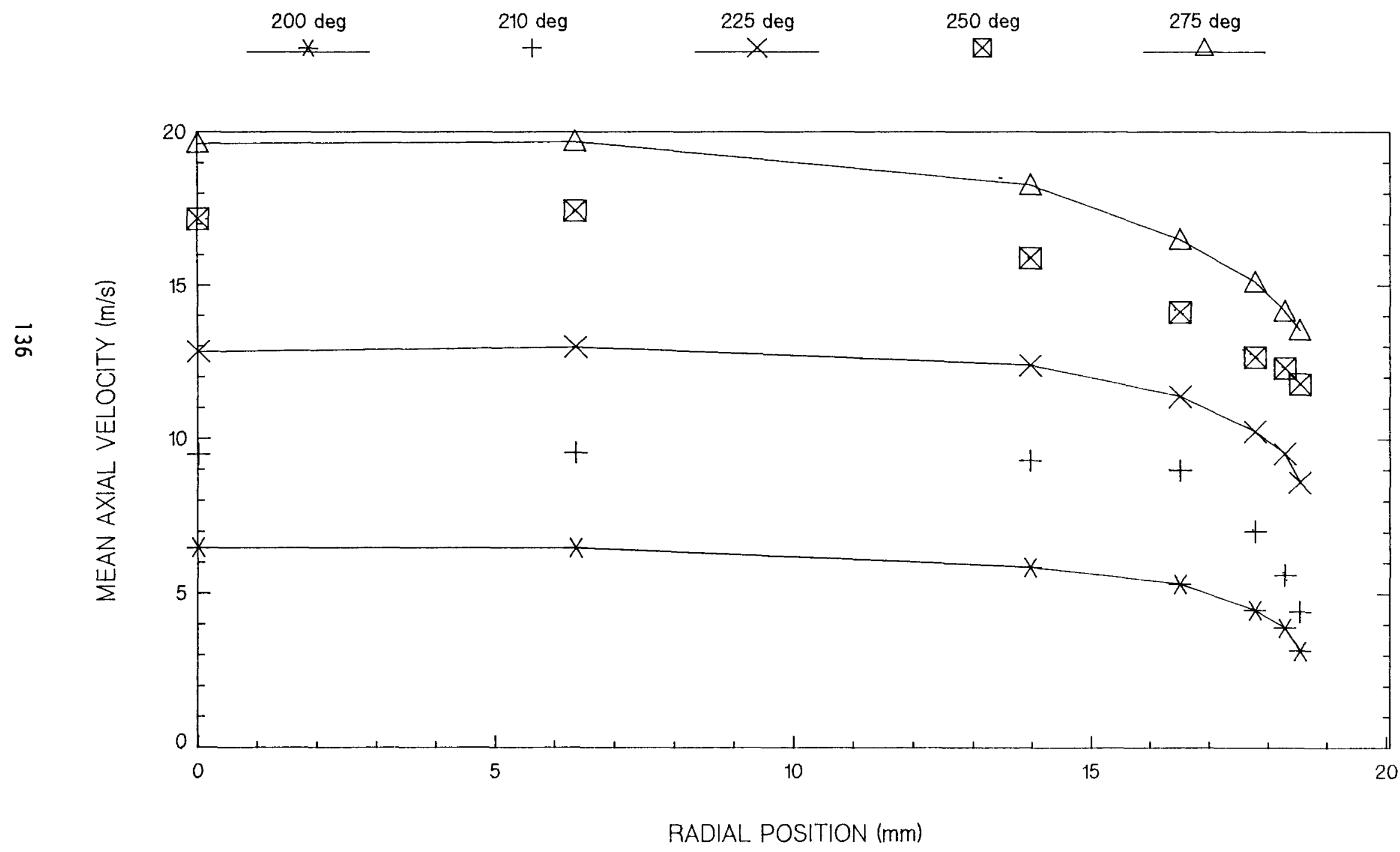

FIGURE 5.30 


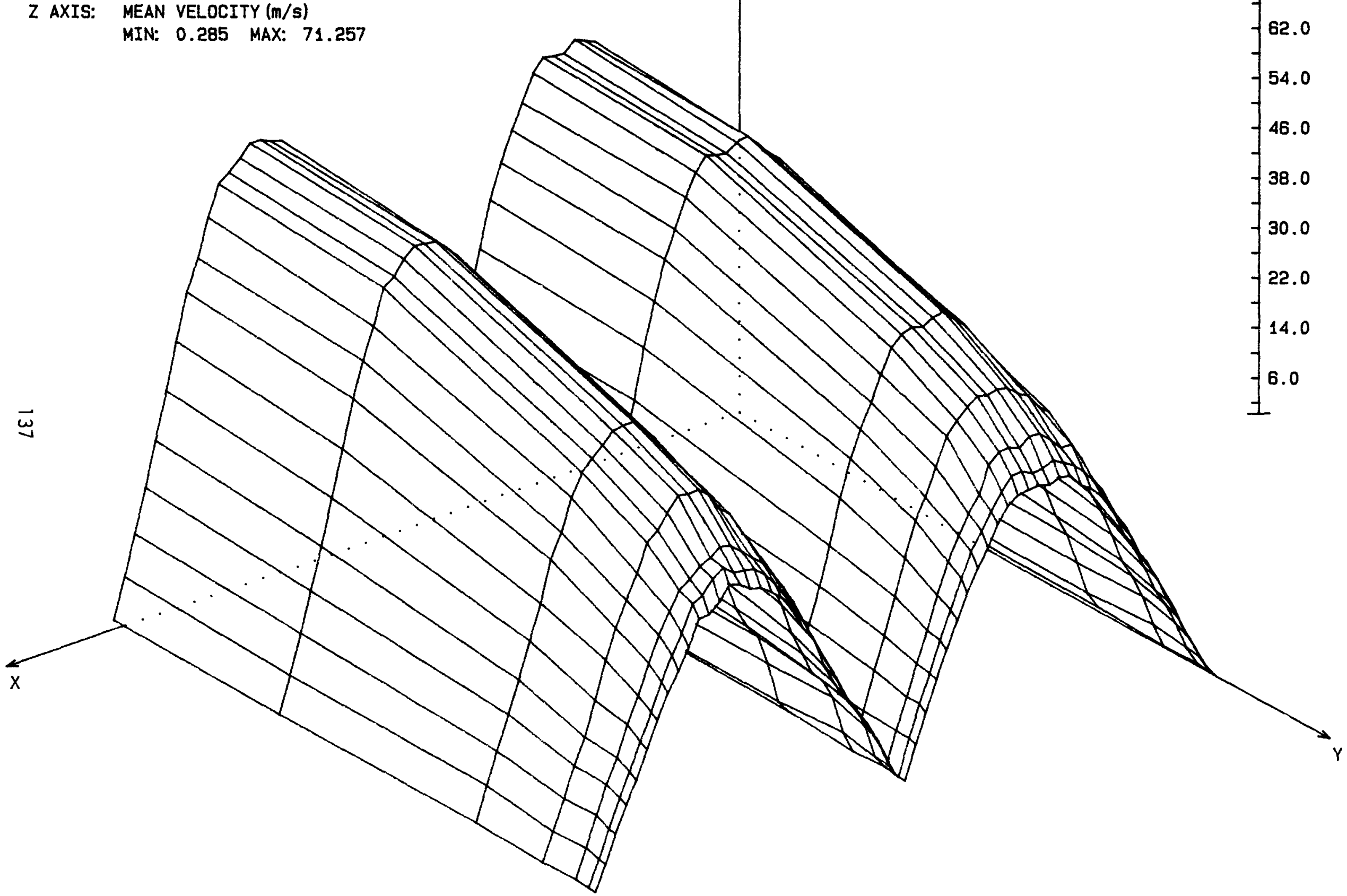

FIGURE 5.31 EXP. RADIAL PROFILE DF MEAN AXIAL VEL. AT 1304mm: $33.5 \mathrm{rpm}$ 


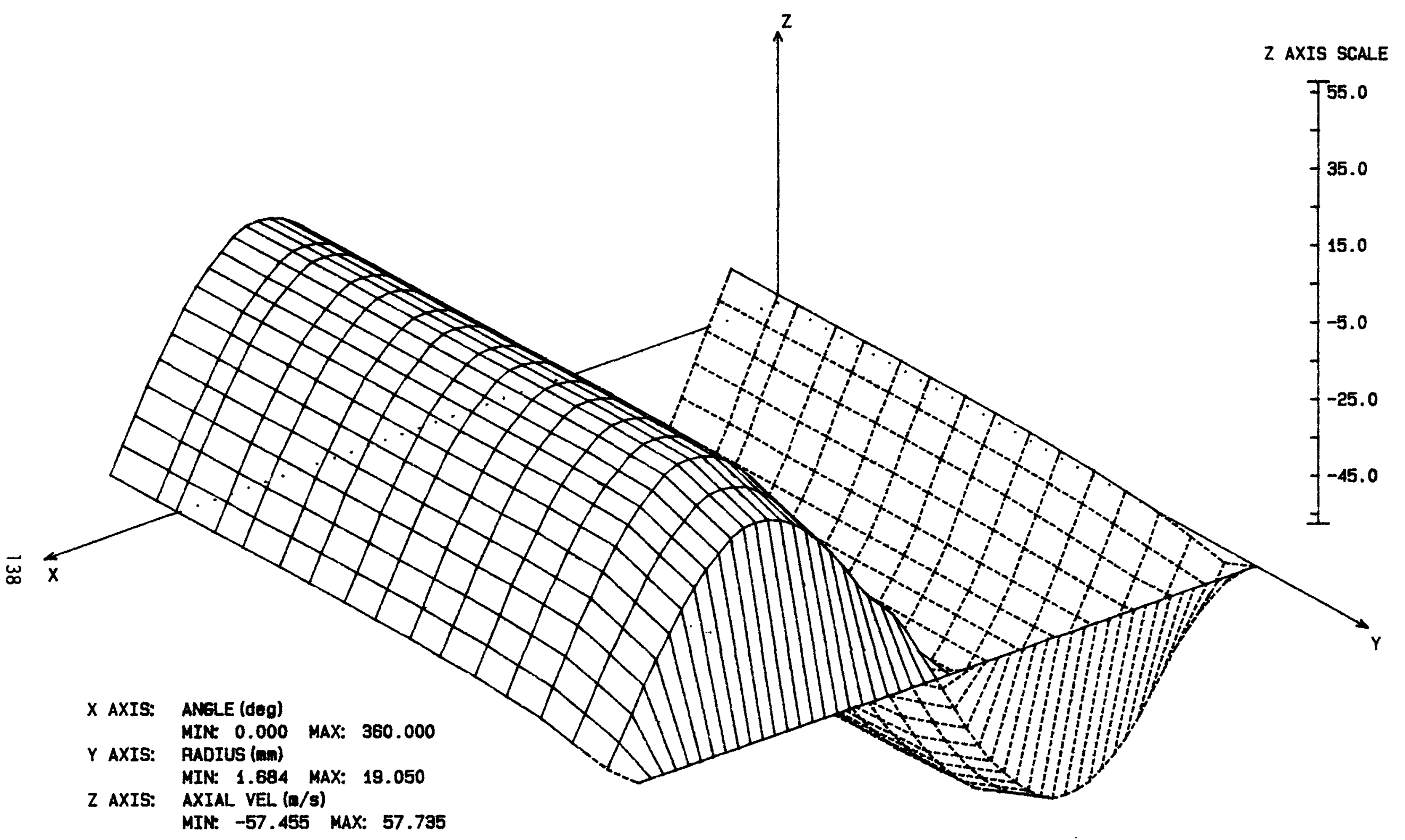

FIGURE 5.32 SIMUL. RADIAL PROFILE OF MEAN AXIAL VEL. AT 1304mm: $33.5 \mathrm{rpm}$ 


\section{SIMULATED RADIAL PROFILES OF MEAN AXIAL VELOCITY}

RIG SPEED: $33.5 \mathrm{rpm}$ PROBE POSITION: $1304 \mathrm{~mm}$

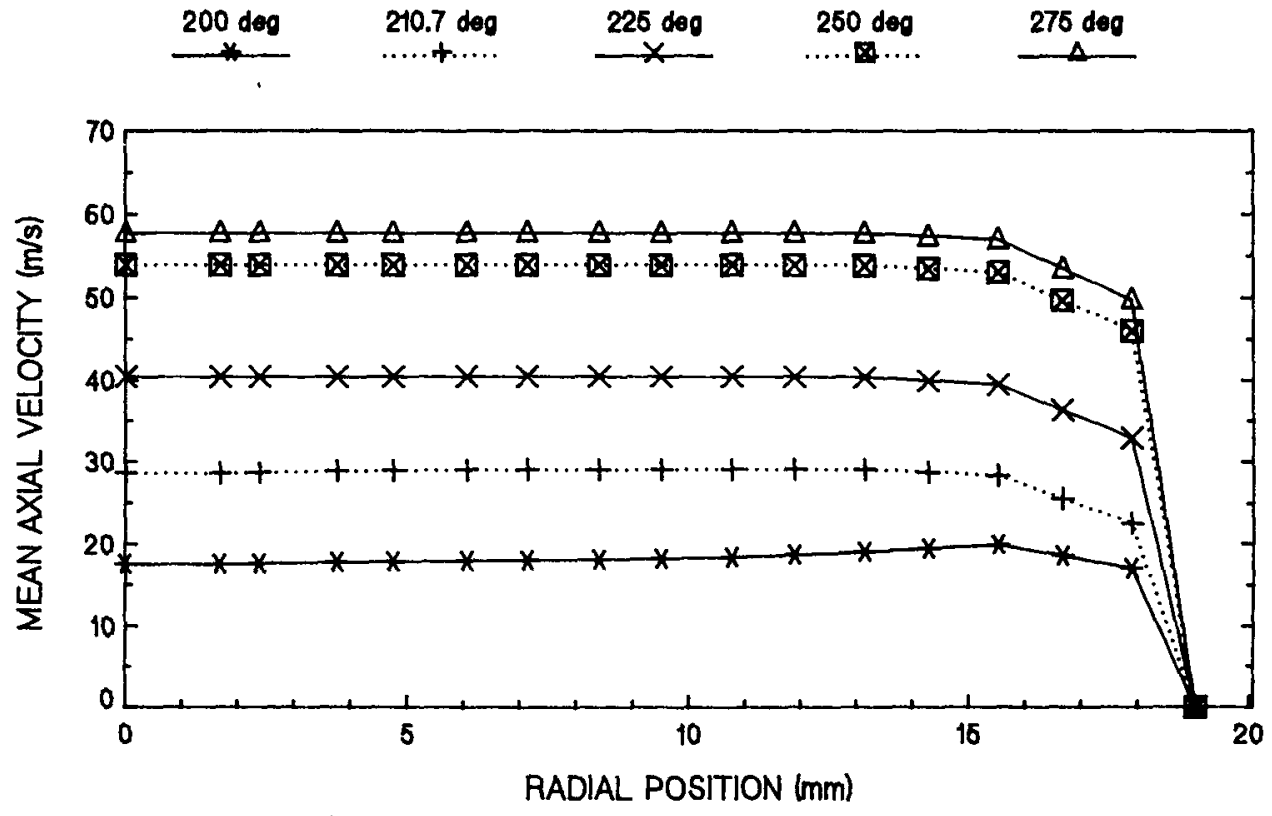

Figure 5.33

EXPERIMENTAL RADIAL PROFILES OF MEAN AXIAL VELOCITY RIG SPEED: $33.5 \mathrm{rpm}$ PROBE POSITION: $1304 \mathrm{~mm}$

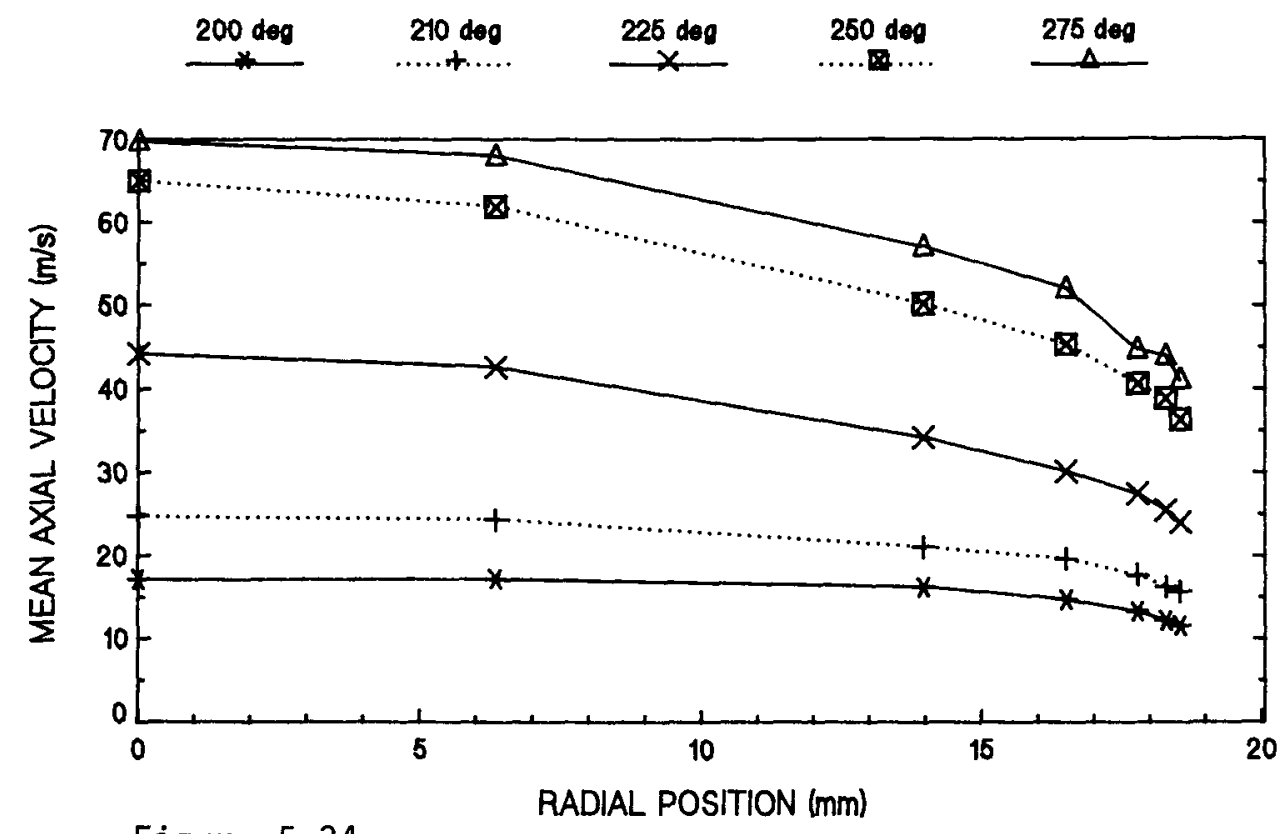

Figure 5.34 


\section{COMBINED RADIAL PROFILES OF MEAN AXIAL VELOCITY}

RIG SPEED: $33.5 \mathrm{rpm}$ PROBE POSITION: $1304 \mathrm{~mm}$

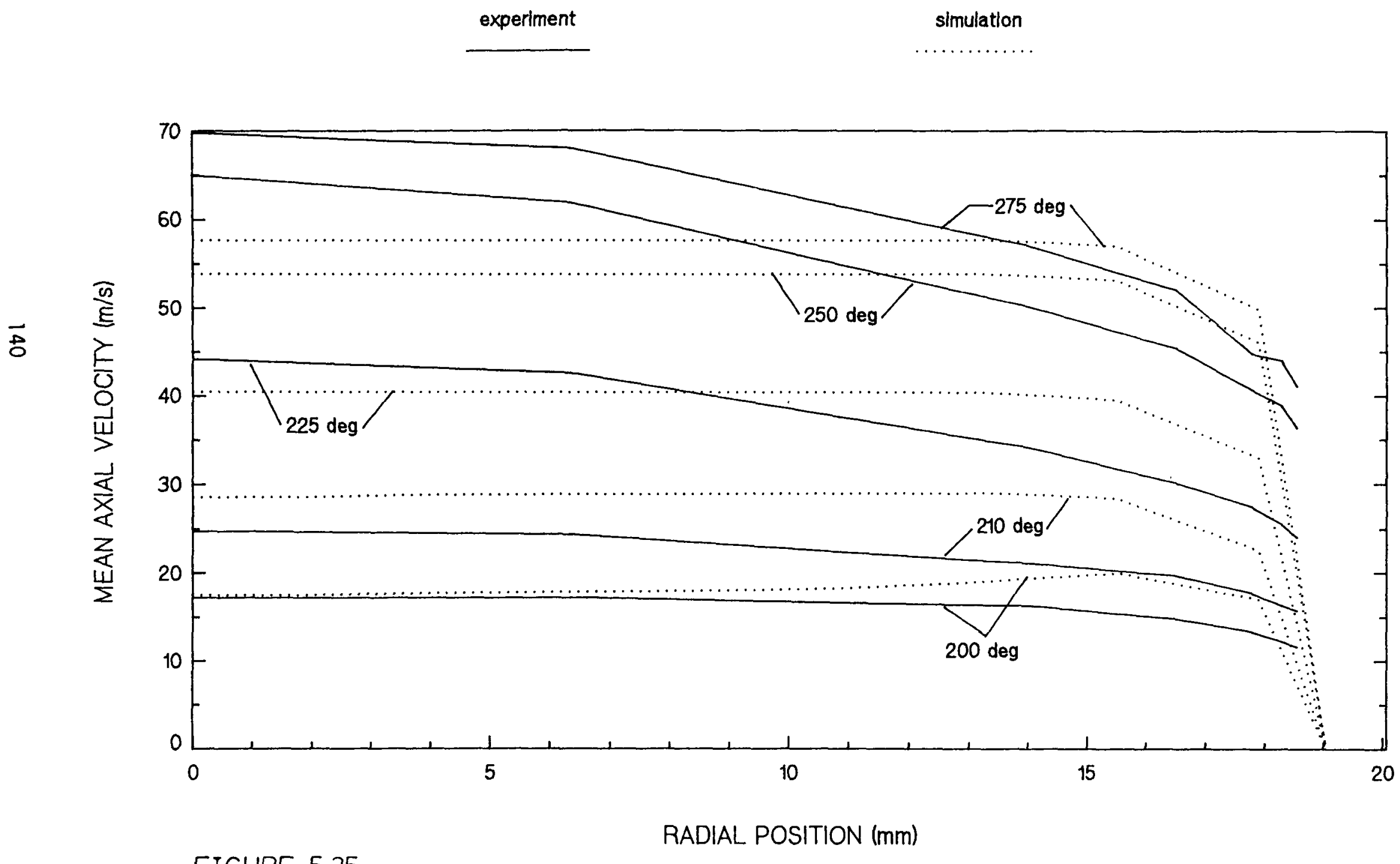

FIGURE 5.35 
$\left(x 10^{\wedge} 3\right)$

$Y$ AXIS: RADIUS (mm)

MIN: 1.684 MAX: 19.050

$Z$ AXIS: RADIAL VEL $(\mathrm{m} / \mathrm{s})$

MIN: -0.011 MAX: 0.006

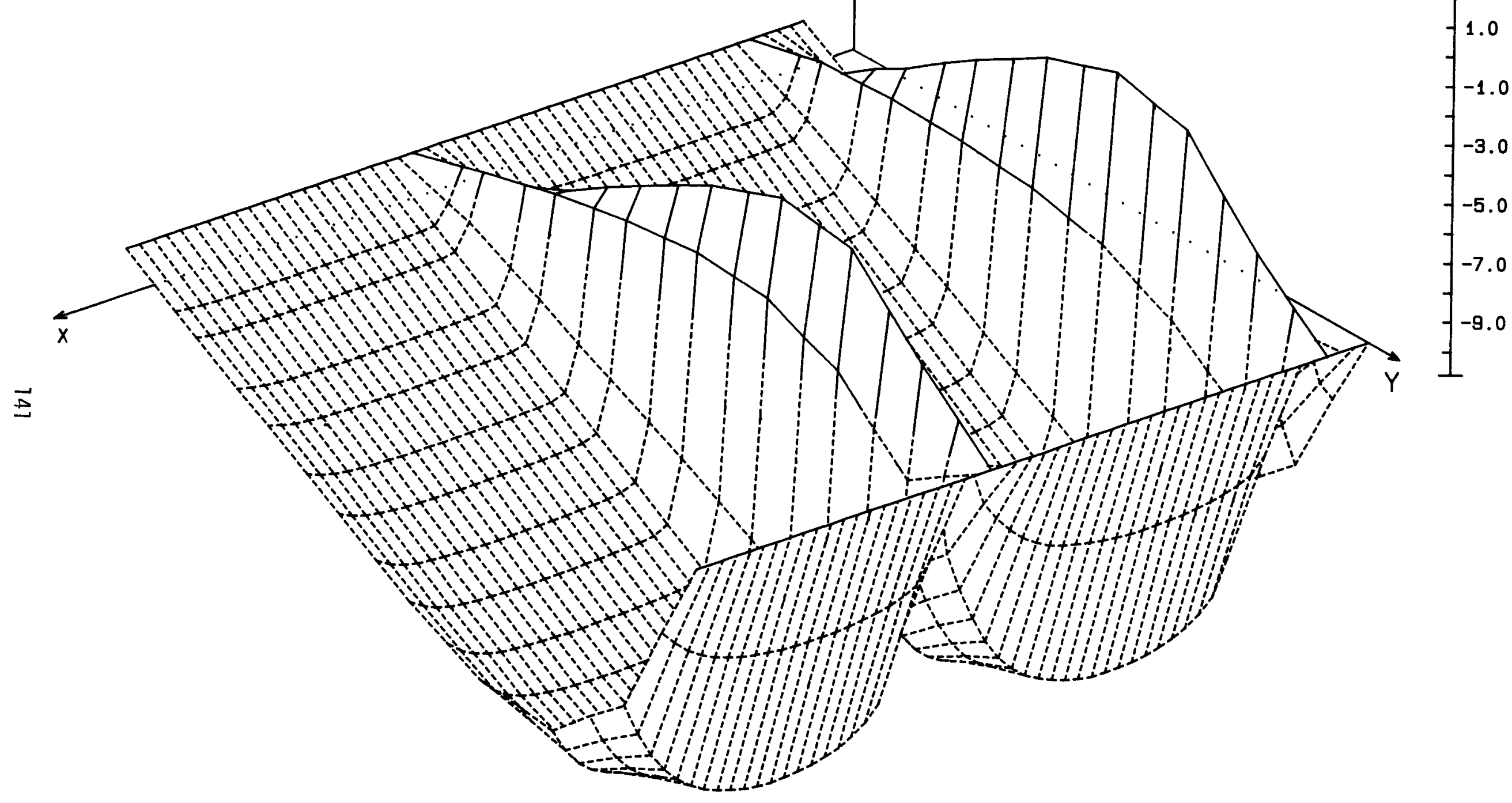

FIGURE 5.36 SIMUL. RADIAL PROFILE OF MEAN RADIAL VEL. AT 1304mm: 33.5rpm 


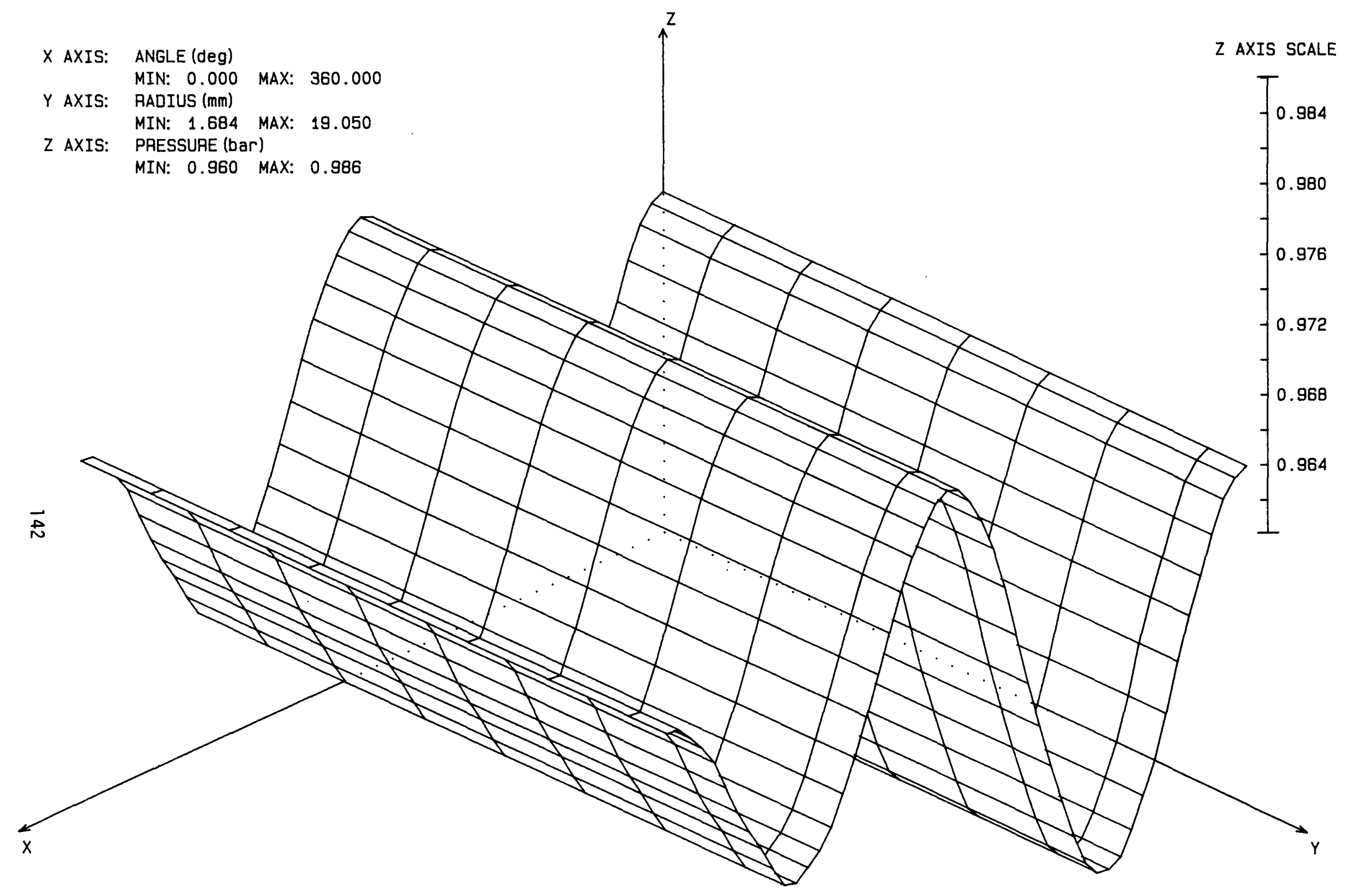

FIGURE 5.37 SIMUL. RADIAL PRESSURE PROFILE AT 1304mm: $33.5 \mathrm{rpm}$ 
piston leakage effects) as expected from the bulk velocity profiles shown in figure 5.13 ; in other words, the experimental and simulated results are consistent on a mass conservation basis.

The absence of any discernable radial pressure gradient in figure 5.37 is in accordance with the smallness of the radial velocities displayed by figure 5.36. The behavior revealed by figure 5.36 (as well as by figure 5.27 at $11.3 \mathrm{rpm}$ ) does not appear to be in conflict with the experimental data, namely, a radial flow reversal corresponding with the axial flow reversal at $0^{\circ}$ and $180^{\circ}$ (see figures 5.23 and 5.32). The extent to which this radial flow reversal is important in the laminar/turbulent flow transition process should be an important ingredient of future simulation and experimental investigations.

\subsubsection{Simulated Cyclic Performance}

An overview of the simulated cyclic performance of the test $\mathrm{rig}$ is given by table 5.3. The 'net kinetic energy conversion' is the sum of the irreversible kinetic/thermal energy conversion (or dissipation) and the isentropic heat generation rate (or v. $\nabla P$ 'work'). A positive value indicates that over the cycle, there is a net conversion of kinetic into thermal energy; a negative value indicates the reverse. Negative 'indicated work done' denotes that work is done on the fluid by the piston.

Table 5.3 Base case cyclic energy balances.

\begin{tabular}{llccccc}
\hline $\begin{array}{l}\text { Engine } \\
\text { Speed } \\
(\mathrm{rpm})\end{array}$ & $\begin{array}{l}\text { Simul } \\
\text { Code }\end{array}$ & $\begin{array}{c}\text { Net Heat } \\
\text { Input } \\
(\mathrm{J})\end{array}$ & $\begin{array}{c}\text { Net Heat } \\
\text { Output } \\
(\mathrm{J})\end{array}$ & $\begin{array}{l}\text { Net Kinetic } \\
\text { Energy Con- } \\
\text { version(J) }\end{array}$ & $\begin{array}{c}\text { Indicated } \\
\text { Work Done } \\
(\mathrm{J})\end{array}$ & $\begin{array}{l}\text { Energy } \\
\text { Balance } \\
\text { Error(8) }\end{array}$ \\
\hline 11.3 & $1-\mathrm{d}$ & 3.3101 & 23.1855 & -0.0165 & -19.8918 & 0.000065 \\
& $2-\mathrm{d}$ & 1.2399 & 13.5442 & 3.887 & -8.4173 & 0.000241 \\
\hline 33.5 & $1-\mathrm{d}$ & 20.8935 & 155.8568 & -0.0259 & -134.9901 & 0.000534 \\
& $2-\mathrm{d}$ & 6.5336 & 45.4136 & -1.4571 & -40.332 & 0.006644 \\
\hline
\end{tabular}

The magnitude of the difference in dissipation modelled by the one- and twodimensional codes is manifested by the large difference in indicated work predictions. At $11.3 \mathrm{rpm}$, the one-dimensional code predicts an indicated work input 2.4 times greater than the two-dimensional code, while at $33.5 \mathrm{rpm}$, the one-dimensional prediction is 3.3 times greater. The net heat output required to balance the indicated work input follows this trend while the net heat input is adjusted to close the energy balance. The two-dimensional net kinetic energy conversion values are large relative to their one-dimensional counterparts. This also is consistent with the under-prediction of 
dissipation in the two-dimensional code owing to the neglect of turbulence in the test section. All the energy balance errors are less than .018 at worst with the worst error being achieved after 3 cycles from rest.

\section{4 .7 Closure}

The salient conclusion to be drawn from the comparison of the baseline simulated and experimental results is that the two-dimensional simulation should include an adequate turbulence model in order to match the experimental data. The major physical impact of the measured turbulence appears to be a large increase in the radial advection fluxes in the test section compared with those generated under assumed laminar flow conditions. The effectiveness of the 'turbulence model' used in the one-dimensional code (that is, the pseudo-steady-state friction factor correlation) in replicating experimental conditions cannot be judged definitively due to the absence of adequate measurements. At the very least, such measurements should include ambient pressure and temperature as well as a transient pressure drop profile across the test section.

However, the comparisons performed appear to confirm that the use of the pseudo-steady-state friction factor approach to dissipation modelling is at least an approximation that produces the correct qualitative trends in the simulated results. The importance of correct boundary condition modelling as an integral part of the two-dimensional test section simulation is also highlighted. Hence, it is doubtful whether generic two-dimensional codes that just simulate the test section can be expected to yield good quantitative answers which may be generalised to Stirling machine heat exchangers with arbitrarily complex boundary conditions.

In terms of practical design application, these results point to the continued efficacy of a one-dimensional system analysis of Stirling machine fluid dynamics. The simulation/experimental result comparison shows that there is promise for modifying the pseudo-steady-state approach to reflect adequately the turbulence effects which, based on these data, seem to be a major cause of some of the anomalies in one-dimensional simulation so often cited in the literature.

\subsection{COMPARISON CASE SIMULATION APPLICATION}

The METR experimental parameters chosen for the development of the turbulence model are selected to represent the heater of the Space Power Demonstrator Engine (SPDE) operating at relatively high power. These parameters are shown in table 5.4 . 
Table 5.4 Comparison case experimental parameters.

Operating point: MTI Experimental test no. 42 of 9/11/86

Experimental indicated power: $17.9 \mathrm{~kW}$

Max. Reynolds number within the heater over a cycle: 13,985.3

Maximum Valensi number within the heater over a cycle: 91.3

Minimum Valensi number within the heater over a cycle: 71.7

Maximum heater bulk Reynolds number over a cycle: 11,706.4

Maximum heater bulk Valensi number over a cycle: 86.9

Minimum heater bulk Valensi number over a cycle: 72.2

where:

$\left(N_{R e}\right)_{b u l k}=d \Sigma(g V) / \Sigma(\mu V)$

$\left(N_{V a}\right)_{b u 1 k}=.5 \pi f d^{2} \Sigma(M) / \Sigma(\mu V)$

Two sets of Reynolds and Valensi numbers are defined. One set corresponds to the maxima and minima occurring within the heater while the second set defines 'bulk' values, namely those obtained from volume averages over the entire heater (equations (5.1) and (5.2)). In terms of the METR operating philosophy, the rig parameters were chosen to reflect the bulk Reynolds and Valensi numbers with the latter taken as the mean of the minimum and maximum values.

The adjusted rig parameters for the comparison case are noted in table 5.5. The chief differences from the baseline case occur in the expansion space, conical transition piece, and flexible joint parameters. The test section length is reduced by $323 \mathrm{~mm}$ and a 'transition length' within the test section has been defined to contain the transition from a one - to a twodimensional spatial discretisation. Previously, this transition was contained within the nozzle and hence occurred under axially varying flow area conditions. Placing the transition in the constant diameter test section is analytically preferable although no discernable numerical differences can be detected.

\subsection{THE TURBULENCE MODEL}

The only difference between the equation set used in the baseline and comparison cases is that the Reynolds stress tensor and turbulent heat flux terms have been reintroduced into the momentum and thermal energy equations respectively (equations $(2.42 .1$ ) and $(2.43 .1)$ ).

The six restrictions constituting the turbulence model are listed in section 2.3. However, of principal concern in this discussion is the selection of a model fulfilling the stipulation of restriction $V$. Currently, the relevant turbulence terms in the momentum and thermal energy equations are represented by pseudo-steady-state friction factor and heat transfer 
coefficient correlations. The purpose here, therefore, is to replace these correlations with a more fundamental turbulence model.

The selection of the turbulence model is based upon the following criteria:

a. General coordinate and dimensional invariance.

Essentially, this means that the form of the model should be independent of the spatial discretisation scheme and system of units used. Casting the model in tensor form and using dimensionless constants meets this criterion.

b. Ful1 realizability (Lu78).

In particular, this requires that all the components of the turbulent kinetic energy are non-negative for any possible flow condition. Further, full realizability means that the turbulence model must be capable of evolving positive turbulent kinetic energy from a rest state (that is, zero momentum) without the necessity of defining an artificial rest-state positive turbulence field. This is particularly important under oscillating flow conditions during which repeated laminar-to-turbulent transitions occur, since under these circumstances, numerically illconditioned models show steady-state solutions that are dependent on the initial conditions. However, physical realizability alone is insufficient in a numerical context since models that are physically realizable can still produce negative turbulent kinetic energies due to the errors inherent in a discrete numerical process, particularly in laminar-to-turbulent transitions. Thus the model must also be numerically realizable in the sense that its numerical or discrete analog will also yield non-negative turbulence kinetic energies under all flow conditions.

c. Material frame indifference.

This requires that the form of the Reynolds stress term (equation $(2.42 .2)$ ) in a two-dimensional turbulence field be the same 'in all non-inertial frames of reference which can undergo arbitrary time-dependent rotations and translations relative to an inertial framing' (Sp87). Basically, this implies that if the Reynolds stress is dependent upon the mean velocity gradient tensor $\nabla v$, 
Table 5.5 METR comparison simulation input parameter set.

\begin{tabular}{|c|c|}
\hline $\begin{array}{l}\text { Expansion Space } \\
\text { Piston stroke (mm) } \\
\text { Piston end cap thickness (mm) } \\
\text { Piston end cap taper (deg) } \\
\text { Clearance length at piston TDC (mm) } \\
\text { Cylinder diameter (mm) } \\
\text { Angular velocity (rpm) }\end{array}$ & $\begin{array}{c}251.46 \\
0 . \\
90 . \\
67.9 \\
127 . \\
33.75\end{array}$ \\
\hline Conical Transition Piece & \\
\hline $\begin{array}{l}\text { Axial length }(\mathrm{mm}) \\
\text { Cylinder mating diameter }(\mathrm{mm}) \\
\text { Flexible joint mating diameter }(\mathrm{mm}) \\
\text { \# of control volumes }\end{array}$ & $\begin{array}{l}219.1 \\
127.0 \\
127.0 \\
3\end{array}$ \\
\hline $\begin{array}{l}\text { Flexible Joint } \\
\text { Cylinder side axial length(mm) } \\
\text { Cylinder side diameter(mm) } \\
\text { Cylinder side \# of control volumes } \\
\text { Nozzle side axial length(mm) } \\
\text { Nozzle side diameter(mm) } \\
\text { Nozzle side \# of control volumes } \\
\end{array}$ & $\begin{array}{c}15.875 \\
152.4 \\
2 \\
73.025 \\
139.7 \\
2 \\
\end{array}$ \\
\hline $\begin{array}{l}\text { Spacer } \\
\text { Axial length(mm) } \\
\text { Cylinder side flange length }(\mathrm{mm}) \\
\text { Nozzle side flange length }(\mathrm{mm}) \\
\text { Diameter }(\mathrm{mm}) \\
\text { Cylinder side flange diameter }(\mathrm{mm}) \\
\text { Nozzle side flange diameter }(\mathrm{mm}) \\
\text { \# of control volumes }\end{array}$ & $\begin{array}{r}63.5 \\
3.175 \\
6.35 \\
139.7 \\
127.0 \\
127.0 \\
3\end{array}$ \\
\hline $\begin{array}{l}\text { Heat Exchanger } \\
\text { Axial length }(\mathrm{mm}) \\
\text { Cylinder side flange length }(\mathrm{mm}) \\
\text { Nozzle side flange length }(\mathrm{mm}) \\
\text { Casing diameter }(\mathrm{mm}) \\
\text { Hydraulic diameter }(\mathrm{mm}) \\
\text { Flow area ratio } \\
\text { \# of control volumes }\end{array}$ & $\begin{array}{l}50.8 \\
9.525 \\
12.7 \\
127.0 \\
2.1824 \\
.556 \\
4\end{array}$ \\
\hline $\begin{array}{l}\text { Nozzle } \\
\text { Size (1-small, 2-large) } \\
\text { \# of half-nozzle control vols }\end{array}$ & $\begin{array}{l}1 \\
3\end{array}$ \\
\hline $\begin{array}{l}\text { Test Section } \\
\text { External diameter(mm) } \\
\text { Axial length(mm) } \\
\text { Transition length (diameters) } \\
\text { \# of axial control volumes (4 minimum) } \\
\text { \# of radial control volumes (2 minimum) } \\
\text { Wall conductivity }(\mathrm{W} / \mathrm{m}-\mathrm{K}) \\
\text { Wall density }\left(\mathrm{kg} / \mathrm{m}^{\wedge} 3 \text { ) }\right. \\
\text { Wall specific heat capacity }(\mathrm{J} / \mathrm{kg}-\mathrm{K})\end{array}$ & $\begin{array}{lc}: & 50.8 \\
: & 2286.0 \\
: & 2 \\
: & 11(2-\mathrm{d}) \\
: & 8 \\
: & 1.0211 \\
: & 2466.84 \\
: & 753.624\end{array}$ \\
\hline $\begin{array}{l}\text { orking Fluid } \\
\text { Working fluid (1-Air, } 2=\mathrm{Helium}, 3=\mathrm{Hydr}\end{array}$ & \\
\hline
\end{tabular}


then $\nabla v$ must always be non-zero in a two-dimensional turbulence field and a transformation tensor must exist such that:

$\nabla^{*} \mathbf{v}^{*}=\nabla v+$ dual $*$

where ${ }^{*}$ indicates the target non-inertial frame of reference.

A fourth (although non-rigorous) selection criterion is that the model should be amenable to the induction of and the response to temporal discontinuities. This arises because of the nature of the turbulence triggering occurring in oscillating flows. An example of such a discontinuity is evident in figure 5.38 (Se88), which is the closest test point in Seume's data set to the conditions of table 5.4. The abrupt onset of turbulence at $255^{\circ}$ and to a lesser extent at about $20^{\circ}$ as well as the decay of turbulence at $303^{\circ}$ point to discontinuous phenomena. In this case, Seume postulates that the turbulence is triggered advectively and decays rapidly after passage of a turbulent fluid slug. In contrast, Hino et al. (HK83) concluded from their oscillating flow experimental data taken in a rectangular duct that turbulence is generated 'explosively' at the onset of flow deceleration, which suggests that local fluid acceleration is the trigger mechanism. Hence in this case as we11, the turbulence triggering is a discontinuous event.

The approach decided upon for implementing these empirically observed discontinuities in the model is via a foldback function formulation. This formulation has the advantage of introducing and controlling discontinuities by continuously changing selected parameters, an ideal characteristic for a numerical implementation. An example of a simple foldback function is given in figures 5.39 to 5.42. Consider a parabolic function of the form:

$y=a x^{2}+b x=f(x)$

Hence, initially if:

$y_{1}=a x_{0}^{2}+b x_{0}=f\left(x_{0}\right)$

then, if $y_{1}$ is folded back into $f(x)$, that is:

$y_{2}=f\left(y_{1}\right)=a y_{1}^{2}+b y_{1}$

equations (5.4) represent a foldback function. This function is depicted in figure 5.39 where the dashed line represents the foldback process as the output of the function is reflected by the $45^{\circ}$ line back into the function input. Hence if $a=-1$ and $b=3$, the function is 'ordered' since the solution converges to a unique value. Setting $b=4$ produces the effect of 

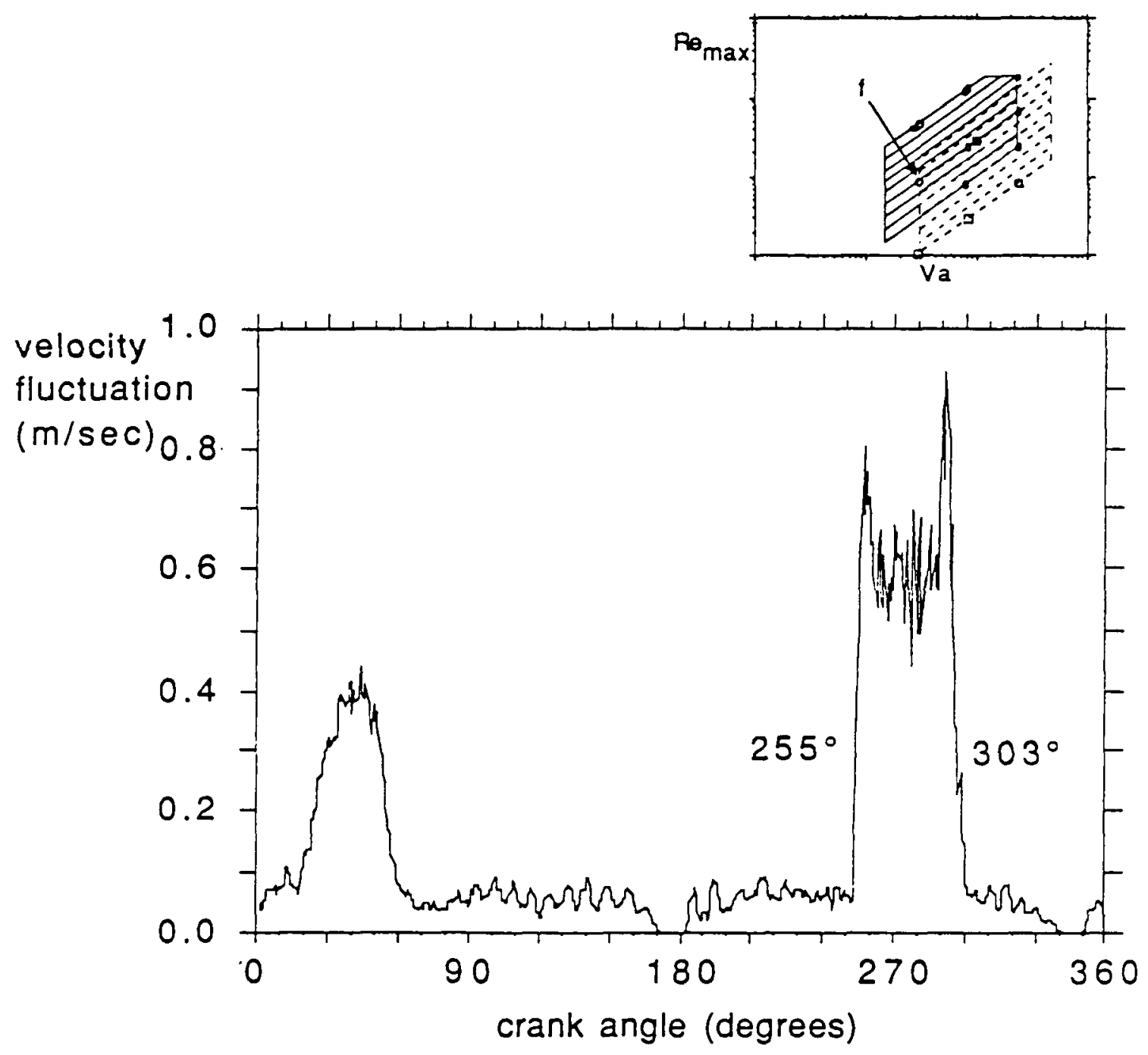

Velocity fluctuation near the open end case $f$

$$
\begin{array}{ll}
x / d=58 & \left(N_{R e}\right)_{\text {max }}=8630 \\
l / d=60 & \\
r / R=0.96 & N_{V a}=28.8
\end{array}
$$

Figure 5.38 (Extracted from reference Se88) 


\section{ORDERED CONDITION$$
y=-x^{\wedge} 2+3 x
$$

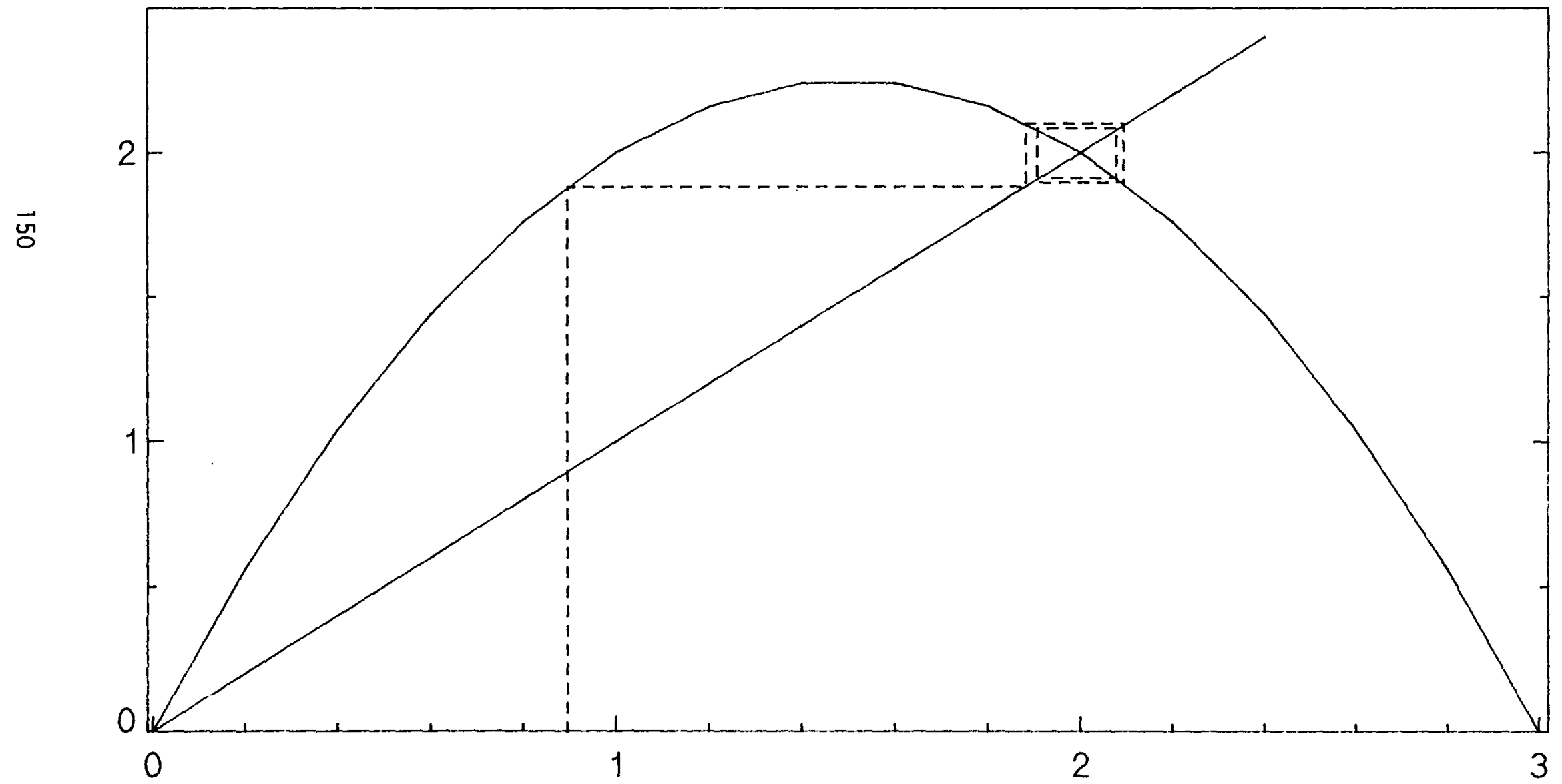

FIGURE 5.39 


\section{STABLE CONDITION \\ $y=-x^{\wedge} 2+4 x$}

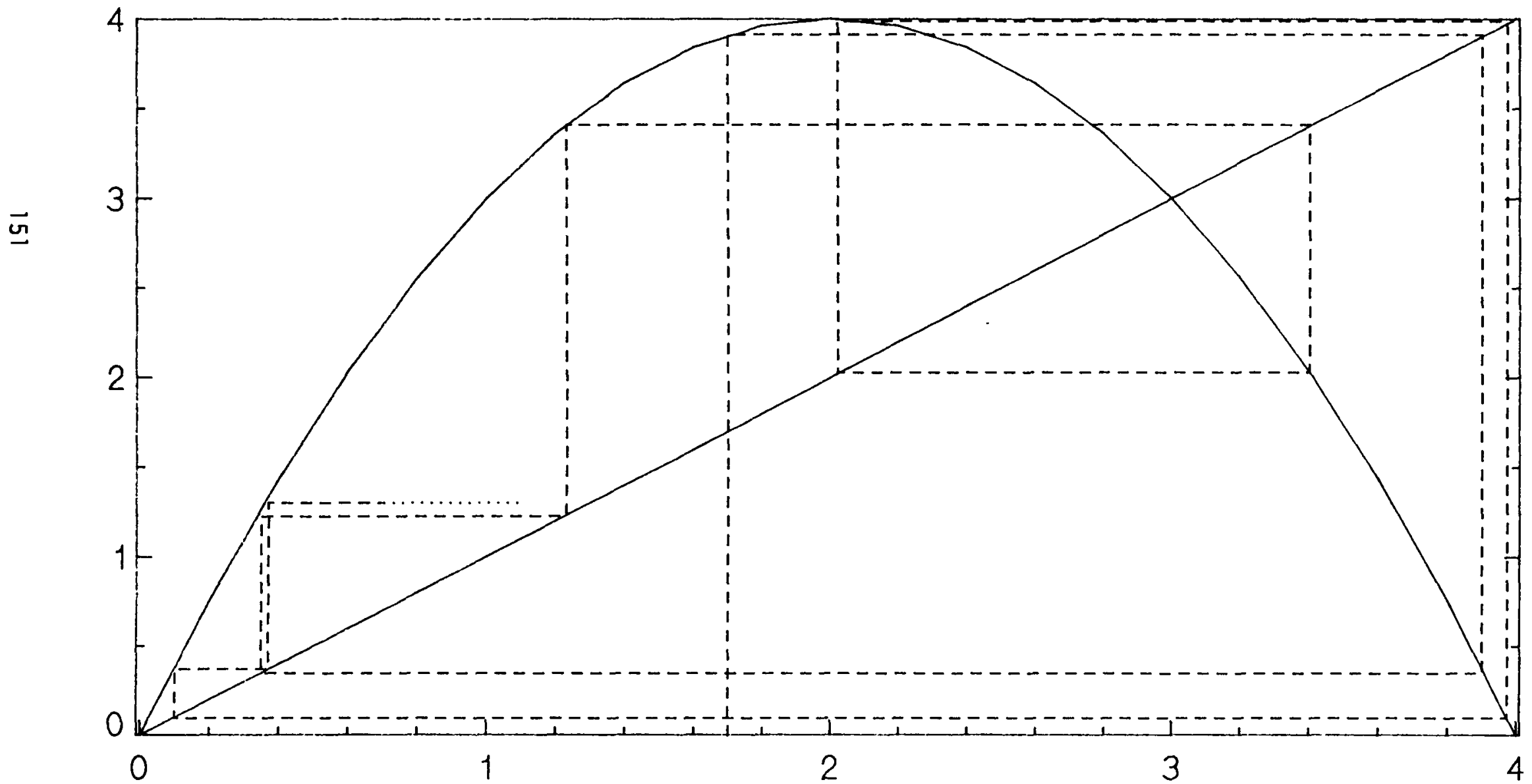

FIGURE 5.40 


\section{TRANSITION CONDITION

$$
y=-x^{\wedge} 2+5 x
$$

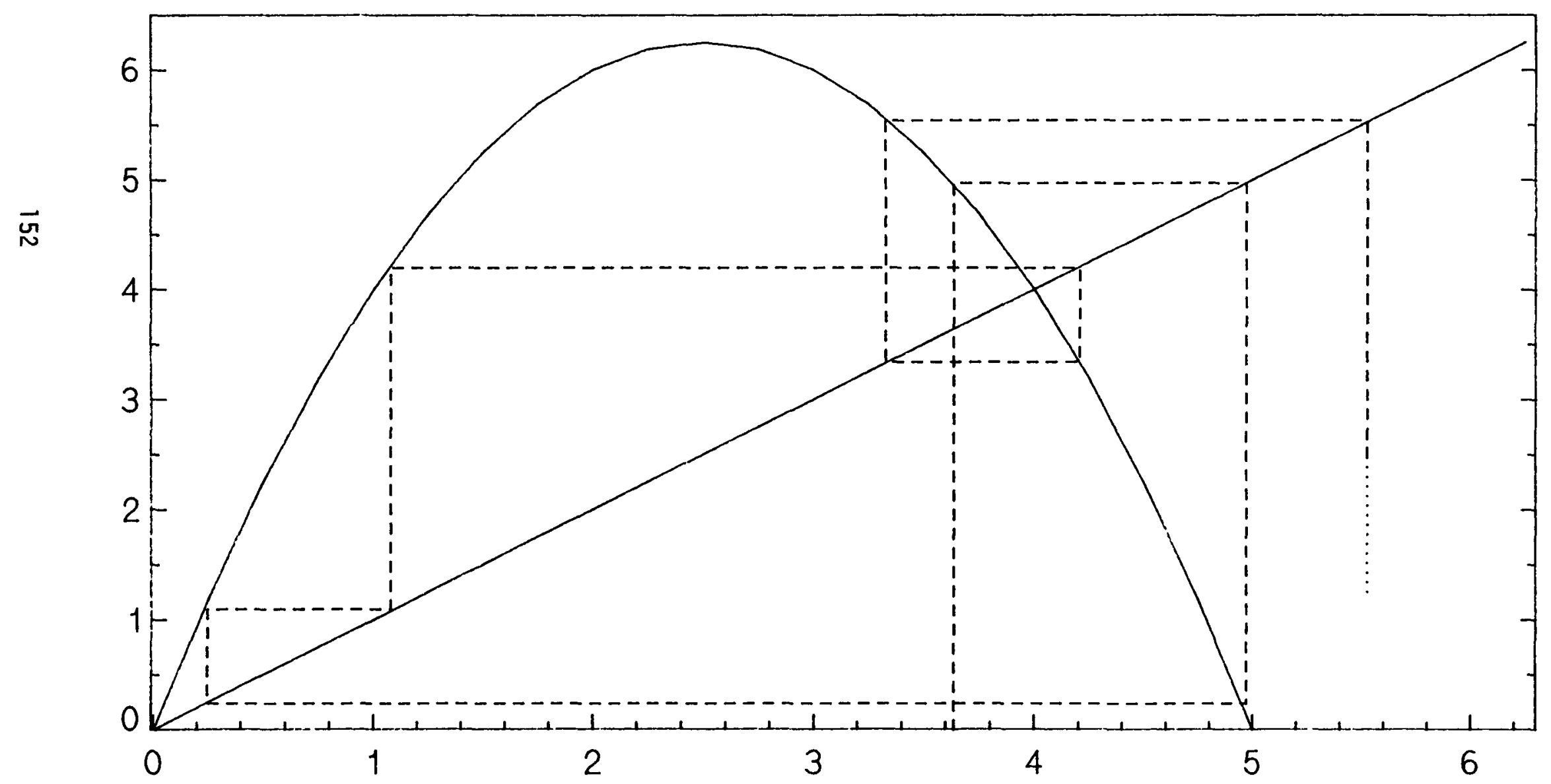

FIGURE 5.41 


\section{CHAOTIC CONDITION

$$
y=-.5 x^{\wedge} 2+5 x
$$

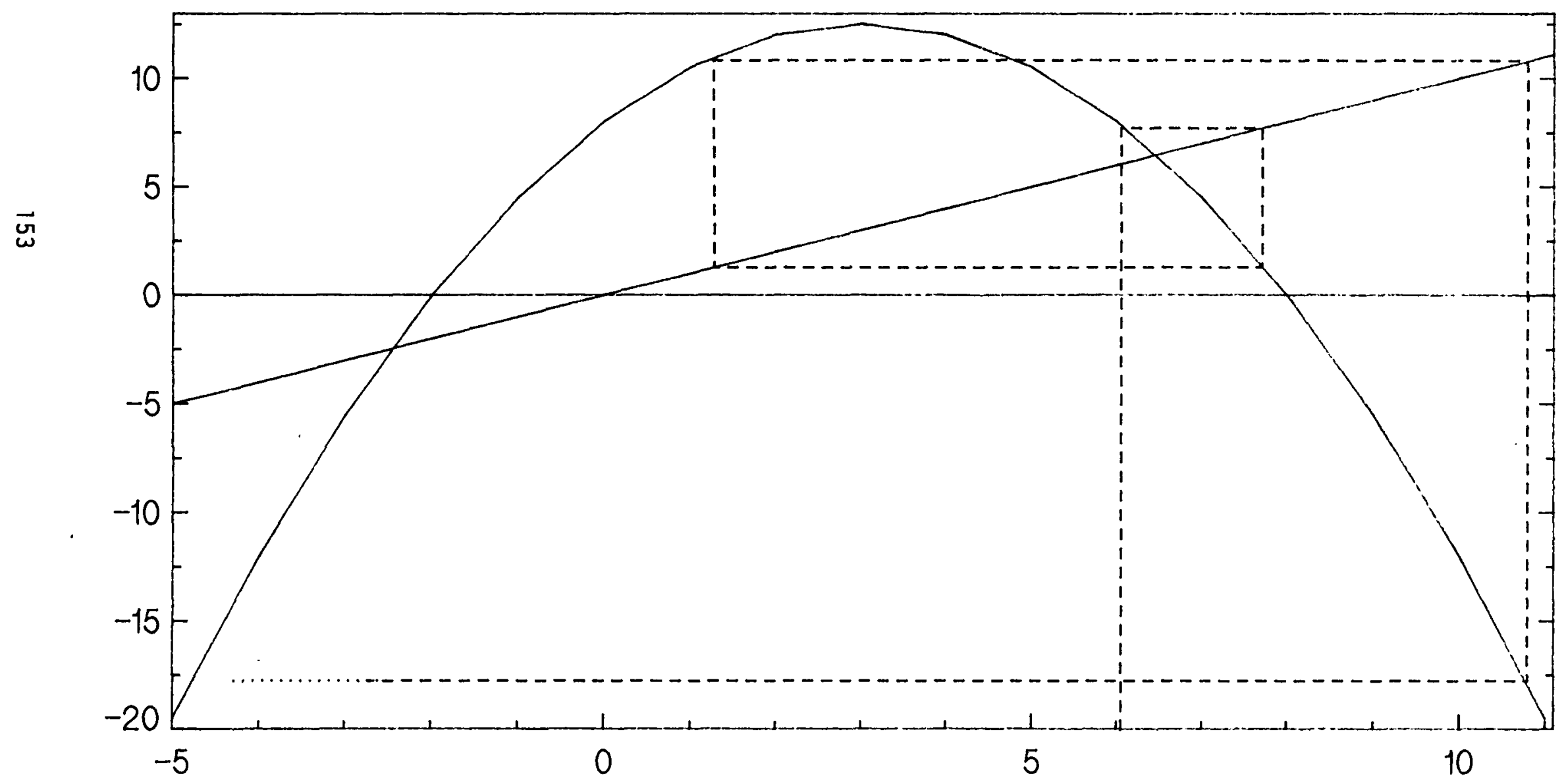

FIGURE 5.42 
figure 5.40, where the solution converges to a 'stable' condition by tracing a closed coordinate path. Increasing $b$ to 5 yields a 'transition' condition in which the solution gradually degenerates into chaos (figure 5.41). Finally, figure 5.42 shows a fully chaotic condition when $a--.5$. Hence a continuous change in $a$ and/or $b$ produces a discontinuous change in the stability of the foldback function so that the chaos 'evolves' in a fashion simulating that observed empirically without creating numerical anomalies.

The foldback function methodology invoked in this analysis was selected initially because it is ideally suited to the iterative structure of the numerical algorithm used (section 2.5), which strongly motivated its initial selection. Yet it must be emphasized that other classes of 'chaos' models (such as fractal methods) are potentially useful if not superior for modelling laminar-to-turbulent (and turbulent-to-laminar) transitions. Time constraints, however, did not permit the investigation of alternates to the foldback function methodology chosen.

\subsubsection{Selection of the Turbulence Model}

Consider the Reynolds or turbulent shear stress term in the momentum equation (2.42.1):

$[v]^{(t)}=-\overline{\left[v \bar{g}^{\prime}[\mathrm{v}]^{\prime}\right.}$

In terms of the rigorous discrete volume analysis used as the basis of the simulation (Go87) together with the six restrictions defining the turbulence model, the ideal choice of turbulence model would be a functional form yielding the correlation on the right-hand side (RHS) of equation (5.5) directly. By a manipulation of the Navier-Stokes equations (Hi75), the exact differential transport equations for the Reynolds stresses may be expressed as (HL72):

$$
\begin{aligned}
& \mathrm{D}\left(\overline{g_{i}^{\prime} v_{j}^{\prime}}\right) / \mathrm{Dt}=-\left(\overline{v_{j}^{\prime} g k} \partial \overline{v_{i}} / \partial \mathrm{x}_{k}+\overline{g_{\text {generation }}^{\prime} v_{k}} \partial \overline{v_{j}} / \partial \mathrm{x}_{k}\right) \\
& -2 \mu \overline{\left(\partial v_{i}^{\prime} / \partial x_{k}\right)\left(\partial v_{j}^{\prime} / \partial x_{k}\right)}+P^{\prime} \overline{\left(\partial v_{i}^{\prime} / \partial x_{j}+\partial v_{j}^{\prime} / \partial x_{i}\right)} \\
& -\partial\left[\overline{g_{i}^{\prime} v_{j}^{\prime} v_{k}}-\mu\left\{\partial\left(\overline{v_{i}^{\prime} v_{j}^{\prime}}\right) / \partial x_{k}+\overline{P^{\prime}\left(\delta_{j k^{v}}^{\prime}+\delta_{i k_{j}^{\prime} v^{\prime}}\right.}\right] / \partial x_{k}\right.
\end{aligned}
$$

where the subscripts $i, j$, and $k$ imply the usual tensor summations. In its exact form, equation (5.6) is not soluble since the RHS contains a number of new correlations (for example, in the dissipation and diffusion terms) for which closed form determinations must be found, that is, the ubiquitous 
closure problem. Typically, these closures are achieved (for example, see HL72, LR75, GE76) by specifying the unknown correlations in terms of algebraic functions of known correlations, the turbulence kinetic energy $(k)$, and the rate of dissipation of turbulence kinetic energy $(\epsilon)$, which are defined as follows:

$k=0.5\left(\overline{v_{i}^{\prime}}\right)^{2}$

$\epsilon=\nu \overline{\left(\partial v_{i}^{\prime} / \partial x_{j}\right)^{2}}$

Employing these techniques expands equation (5.6) into a set of six equations for the two-dimensional field simulated in the METR test section, namely, four transport equations for the Reynolds stresses and two for $k$ and $\epsilon$, respectively.

However, it is also possible to determine the Reynolds stresses directly from a $k-\epsilon$ equation set using Boussinesq's eddy-viscosity concept, which assumes that the turbulent shear stresses are proportional to the meanvelocity gradients (analogous to the viscous stresses in laminar flows) or:

$\tau_{i j}^{(t)}=\mu^{(t)}\left(\partial \overline{v_{i}^{\prime}} / \partial x_{j}+\partial \overline{v_{j}^{\prime}} / \partial x_{i}\right)-2 k \delta_{i j} / 3$

The turbulent viscosity $\mu^{(t)}$ can be obtained from the Kolmogorov-Prandt 1 expression (Ro84):

$\mu^{(t)}=\rho K k^{2} / \epsilon$

Equation (5.8), however, suffers from a major disadvantage because it yields isotropic principal Reynolds stresses, that is:

$\tau_{i i}^{(t)}=\tau_{j j}^{(t)}=\tau \int_{k k}^{(t)}$

This has the consequence of erroneously predicting uni-directional flows in non-circular ducts as well as producing substantial inaccuracies in the calculation of separated turbulent flows (such as that occurring over a backward facing step). Speziale ( $\mathrm{Sp} 87$ ) has proposed solving this problem by replacing the linear form of equation (5:8) with a non-1inear, quadratic form. He has solved several test problems successfully using this approach.

Therefore, in the context of the METR analysis, since a closure model of the two-parameter or $k-\epsilon$ type is in any event necessary for implementing the desirable full Reynolds stress model, in terms of the imposed temporal and 
fiscal constraints, it was judged prudent to limit the turbulence model in the METR simulation to such a two-parameter model, at least as a first step. Equations (5.8) and (5.9) are thus used to generate the necessary Reynolds stresses. Clearly, the limitations of equation (5.8) must be acknowledged, but, fortunately, they are of second order significance for the constant area, axi-symmetric METR test section to which the turbulence model is being applied in two dimensions. Hopefully, the experience gained with the $k-\epsilon$ class of two-parameter turbulence model as applied to oscillating flows will provide a basis for future work leading to the implementation of a full Reynolds stress model.

\subsubsection{The $k-\epsilon$ Turbulence Mode1}

Examination of the classic $k-\epsilon$ turbulent model provides a convenient starting point for the model selection process. A general form of the $k-\epsilon$ model in common usage is referred to as the 'low Reynolds number' version, which is purportedly valid throughout the laminar, semi-laminar, and turbulent regions of the flow (LS74). Usually, these characteristics are attributed to the model under steady-state flow conditions. It is not clear from the literature whether the model is intrinsically capable of handling general laminar-to-turbulent transitions (including those where the fluid is accelerated from rest), although local turbulent-to-laminar transitions have been modelled (JL72). The general form of the $k-\epsilon$ model also includes wall correction terms not only to account for near-wall effects but also to allow setting $\epsilon=0$ at the wall (JL72). In terms of the turbulence model restrictions, a tensor notation version of the low Reynolds number $k-\epsilon$ model suitable for compressible fluids has been adapted from LS74 and is given by the following differential transport equations:

$$
\begin{aligned}
& \overline{\rho D k} / \mathrm{D} t=\underset{\text { diffusion }}{\nabla \cdot\left\{\left(\bar{\mu}+K_{1} \mu^{(t)}\right) \nabla k\right\}}+\underset{\text { production }}{2 \mu^{(t)}(\bar{D}: \nabla \bar{v})}-\underset{\text { wall correction }}{2 \vec{\mu}\left(\nabla k^{1 / 2}\right)^{2}}-\underset{\text { dissipation }}{\bar{\rho} \epsilon} \\
& \bar{\rho} \bar{D} \epsilon / D t=\nabla \cdot\left\{\left(\bar{\mu}+K_{2} \mu^{(t)}\right) \nabla \epsilon\right\}+K_{3} \epsilon 2 \mu^{(t)}(\bar{D}: \nabla \bar{v}) / k \\
& \text { diffusion production } \\
& -K_{4} \bar{\rho} \epsilon^{2} / k+2 \overline{\mu \mu}{ }^{(t)}\left(\nabla_{1} \cdot \nabla \bar{v}\right)^{2} \\
& \text { destruction wall correction }
\end{aligned}
$$

These equations share a common form having diffusion, production, destruction (or dissipation), and wall correction terms on the RHS. Of particular note is the form of the production terms which are defined by the scalar prod"ct of the deformation tensor (equation 5.11.4) and the velocity gradient. As this product is non-negative under all flow conditions, the creation or evolution of $k$ and $\epsilon$ is dependent on the value of $\mu^{(t)}$, itself a function of $k$ and $\epsilon$ (equation 5.11.7). Hence, the system of equations is 
indeterminate even though it is closed. The balance of the equations filling out the complete model implementation are given by:

$$
\begin{aligned}
& T^{(t)}=2 \mu^{(t)} \bar{D}-2 \bar{\rho} k I / 3 \\
& \bar{D}=0.5\left\{\overline{\nabla v}+(\nabla \bar{v})^{T}\right\}
\end{aligned}
$$

$-\overline{g_{i}^{\prime} v_{j}^{\prime}}=\tau_{i j}^{(t)}$

$\overline{g_{i}^{\prime} T^{\prime}}=\mu^{(t)}\left(\partial \bar{T} / \partial x_{i}\right) / N_{P r}^{(t)}$

$\mu^{(t)}=K_{5} \rho k^{2} / \epsilon$

$\begin{array}{lll}K_{1}=1 & K_{2}=1 / 1.3 \quad K_{3}-1.44\end{array}$

$K_{4}=1.92\left\{1-0.3 \exp \left(-N_{R}^{(t)}{ }^{2}\right)\right\}$

$K_{5}=0.09 \exp \left(-2.5 /\left(1+N_{R e}^{(t)} / 50\right)\right.$

$N_{R e}^{(t)}=\bar{\rho} k^{2} / \bar{\mu} \epsilon$

Equation (5.11.6) defines the method by which turbulent enthalpy transport (equation (2.35)) is determined from the model while the constants $K_{4}$ and $K_{5}$ are dependent on the turbulent Reynolds number (equation (5.11.9)).

Examination of equations (5.11.1) and (5.11.2) reveals that the $k-\epsilon$ model satisfies the first selection criterion (general coordinate and dimensional invariance) while equation (5.11.3) satisfies the third criterion (material frame indifference). However, the second criterion (full realizability) is not satisfied.

First, equations $(5.11 .7)$ and $(\dot{5} .11 .9)$ are indeterminate if $\epsilon-0$, and equation (5.11.2) is indeterminate if $k=0$. Hence the equations are intrinsically incapable of evolving a positive turbulence while either $k$ or $\epsilon$ is zero. The usual methodology for dealing with this situation is simply to pre-initialise the $k$ and $\epsilon$ fields to values that are compatible with the realizability criterion; namely, the source terms in equations (5.11.1) and (5.11.2) should be positive, or:

$$
\left(2 \mu^{(t)}(\bar{D}: \overline{\nabla v})-2 \bar{\mu}\left(\nabla k^{1 / 2}\right)^{2}-\overline{\rho \epsilon}\right\}_{\text {init }}>0
$$


$\left(K_{3} \epsilon 2 \mu^{(t)}(\bar{D}: \nabla \bar{v})-K_{4} \bar{\rho} \epsilon^{2} / k+2 \bar{\mu} \mu^{(t)}\left(\nabla_{1} \cdot \nabla \bar{v}\right)^{2}\right\}_{\text {init }}>0$

In this sense, the $k-\epsilon$ model may be termed 'weakly' realizable.

However, in oscillating flows where $k$ and $\epsilon$ can be zero in the flow core as well as in the laminar boundary layers, such initialisation techniques mitigate against the prediction of strict laminar-to-turbulent transitions (that is, from $k-0$ to $k>0$ ) by the model per se. Furthermore, from a numerical perspective, there does not appear to be a physically credible way of ensuring that the $k$ source terms (LHS of equations (5.12.1)) will always interact with the transport terms in such a way as to unconditionally produce positive values of $k$ when considering the model from a full realizability perspective.

As a result of its system indeterminacy, the $k-\epsilon$ model is suitable for implementation as a foldback function, so fulfilling the fourth selection criterion. This may be readily achieved by casting equations (5.11.1) and (5.11.2) in implicit or advanced time form, thus rendering them naturally amenable to iterative solution. There are then a number of possibilities (notably in the generation terms) for introducing foldback parameters for continuously controlling the triggering and evolution of the turbulence. This capability opened the possibility that full realizability could be achieved for the model when cast in foldback function form, thus warranting a numerical investigation.

After much tedious experimentation, it eventually became clear that the realizability problem proved to be the main detractor in obtaining anything resembling the type of laminar-to-turbulent transition observed empirically by Seume as shown in figure 5.38. The model could be made to 'work', that is, portray discontinuous transitions, by treating the flow as being 'turbulent' throughout the cycle (that is, $k$ and $\epsilon>0$ for all $t$ ) simply by setting $\mu^{(t)}$ 0 when $N_{R e}<N_{R e}(t r a n s i t i o n)$ (taken to be 2,000). Further difficulties were encountered within the coarse grid framework of the discrete volume analysis (eight control volumes radially) as a result of the wall correction terms in equations (5.11.1) and (5.11.2). The spatial discretisation near the wall strongly determines the influence of these terms, thus violating the scalability inherent in the discrete volume analysis. Alternate wall correction procedures such as the logarithmic wall function method (LS74) were ; lso tested with mixed results. Such empirical procedures by definition are not spatially scalable or generalized.

These experiences prompted a search of the literature for alternate twoparameter turbulence models which could meet the full realizability criterion and also avoid the necessity of special wall functions. Accurate prediction of steady-. tate turbulence or turbulent-to-laminar transition phenomena alone (commonly used for validation) was not regarded as a qualifying criterion. 


\subsubsection{The Wilcox $k-\omega$ Turbulence model}

Wilcox (Wi88) has proposed a $k-\omega$ two-parameter turbulence model which, when implemented in a foldback function, nominally comes much closer than the $k-\epsilon$ model to achieving full realizability (which appears to be necessary for modelling discontinuous transitions). Although still not possessing complete system determinacy, the Wilcox $k-\omega$ model does appear to offer a better opportunity of meeting the full realizability criterion than most of the twoparameter models reported (Ro84, TL72). It may be noted that Spalding also published a report on the $k-\omega$ model (Sp72), but it is not known to what extent the Spalding $k-\omega$ model differs from the Wilcox $k-\omega$ model. $\omega$ is referred to as the specific dissipation rate and is related to $\epsilon$ via:

$\omega=\epsilon / K k$

After a series of numerical applications, Wilcox claims that the $k-\omega$ model is 'much more accurate than comparable two-equation models' although such a claim is, of course, disputable. The Wilcox $k-\omega$ model does not include any wall correction terms since the model can be integrated through the viscous sub-layer at the boundary. Hence these characteristics make the $k-\omega$ model an ideal candidate for implementation in a discrete volume analysis.

In terms of the turbulence model restrictions, a tensor notation version of the $k-\omega$ model given in Wi8 8 may be expressed by the following differential transport equations:

$$
\begin{aligned}
& \bar{\rho} \mathrm{D} k / \mathrm{D} t=\nabla \cdot\left\{\left(\bar{\mu}+K_{2} \mu^{(t)}\right) \nabla k\right\}+\left(\mathrm{T}^{(t)}: \nabla \bar{v}\right)-K_{1} \bar{\rho} \omega k \\
& \text { diffusion production dissipation } \\
& \bar{\rho} \mathrm{D} \omega / D t=\nabla \cdot\left\{\left(\bar{\mu}+K_{3} \mu^{(t)}\right) \nabla \omega\right\}+K_{5} \omega\left(\mathrm{T}^{(t)}: \nabla \bar{v}\right) / k-K_{6} \bar{\rho} \omega^{2} \\
& \text { diffusion production destruction }
\end{aligned}
$$

These equations are similar in form to their counterparts in the $k-\epsilon$ model (equations (5.11.1) and (5.11.2)) except that the wall correction terms are absent. Another notable difference is that the production terms are expressed in terms of the Reynolds stress tensor rather than the deformation tensor. By an argument similar to that invoked previously, the $k-\omega$ equation system is also indeterminate in explicit form. The balance of the equations and constants comprising the complete model are given by:

$$
\begin{aligned}
& T^{(t)}=2 \mu^{(t)}\{\bar{D}-(\nabla \bar{v}) I / 3\}-2 \bar{\rho} k I / 3 \\
& \bar{D}=0.5\left(\nabla \bar{v}+(\nabla \bar{v})^{T}\right\}
\end{aligned}
$$


$-\overline{g_{i}^{\prime} v_{j}^{\prime}}=\tau_{i j}^{(t)}$

$\mu^{(t)}=K_{4} \bar{\rho} k / \omega$

$\overline{g_{i}^{\prime} T^{\prime}}=\mu^{(t)}\left(\partial \bar{T} / \partial x_{\dot{i}}\right) / N(t)$

$$
\begin{array}{lll}
K_{1}=0.09 & K_{2}=0.5 & K_{3}=0.5 ; \\
K_{4}=1 & K_{5}=5 / 9 & K_{6}=3 / 40
\end{array}
$$

In this case, none of the constants show a turbulent Reynolds number dependence. However, the methodology employed by Wilcox to derive the model constants (equation 5.14.8) is open to question since it relies partly on numerical evaluation (as opposed to being entirely based on experimental data). This has particular significance in the light of the scalability requirements of a discrete volume application.

The foldback function formulation of the $k-\omega$ model is achieved via the following manipulation. Substituting equation (5.14.6) into equation (5.14.3) yields:

$\mathrm{T}^{(\mathrm{t})}=2 K_{4} \bar{\rho} k(\overline{\mathrm{D}}-(\bar{\nabla} \overline{\mathrm{v}}) \mathrm{I} / 3) / \omega-, \bar{\rho} \bar{\rho} \mathrm{KI} / 3$

Substituting equation (5.15) into the production term of equation (5.14.2) produces:

$\bar{\rho} \mathrm{D} \omega / \mathrm{D} t=\nabla \cdot\left(\left(\bar{\mu}+K_{3} \mu^{(t)}\right) \nabla \omega-K_{6} \bar{\rho} \omega^{2}+2 \bar{\rho} K_{5}\left[\left[K_{4}(\mathrm{D}-(\bar{\nabla} \mathrm{v}) \mathrm{I} / 3)-\omega \mathrm{I} / 3\right]: \bar{\nabla} \mathrm{v}\right]\right.$

Casting equation (5.16) into advanced time (or implicit) form by discretising the LHS and rearranging the source (production and destruction) terms yields:

$$
\begin{gathered}
\bar{\rho} \omega\left[1 / \Delta t+\left\{\left(2 K_{5} I / 3\right): \nabla \bar{v}\right\}+K_{6} \omega\right]-\nabla \cdot\left\{\left(\bar{\mu}+K_{3} \mu^{(t)}\right) \nabla \omega\right\} \\
-\bar{\rho} \omega^{3} / \Delta t+2 \bar{\rho} K_{4} K_{5}[(\bar{D}-(\nabla \bar{v}) I / 3\}: \nabla \bar{v}]
\end{gathered}
$$

Equation (5.17) is fully realizable. It is also determinate if in equation $(5.14 .6)$ : 
$\omega=0 \quad \Rightarrow \quad \mu^{(t)}=0$

Notice then that as long as the velocity gradient $\nabla \bar{v}$ is non-zero, equation (5.18) will evolve positive $\omega$ even when $\omega=0$ regardless of the value of $k$. This implies a much different physical description of turbulence than the $k-\epsilon$ model, which will only evolve positive $\epsilon$ when $k>0$. A determination of which model is physically 'correct' under oscillating flow conditions (particularly during laminar-to-turbulent transitions) requires a level of analysis beyond the scope of this project and a very detailed experimental oscillating flow data base, which is not yet available (at least from the METR). Nevertheless, Wilcox has demonstrated that under steady-state conditions and during turbulent-to-laminar transitions, the $k-\omega$ model is at least as physically 'correct' as the $k-\epsilon$ model. Hence for the sake of expediency, it will be assumed here that the physical implications of equation (5.17) are valid on the basis that the production term of equation (5.17) is a logical consequence of equations (5.14).

Casting equation (5.14.1) in implicit form yields:

$\bar{\rho} k\left(1 / \Delta t+K_{1} \omega\right)-\nabla \cdot\left(\left(\bar{\mu}+K_{2} \mu^{(t)}\right) \nabla k\right)=\bar{\rho} k^{s} / \Delta t+\left(T^{(t)}: \nabla \bar{v}\right)$

Equation (5.19) is also realizable (not as fully as equation (5.17) though) but it still suffers from the system indeterminacy of the $k-\epsilon$ model in that positive $k$ can only be evolved if $k>0$. Hence some additional empiricism is necessary to trigger the creation of turbulence.

\subsection{4 k- $\omega$ Model Enhancement and Foldback Function Parameters}

Dropping the implicit notation for the sake of clarity and transforming equations (5.17) and (5.19) into generalised discrete volume form (see chapter 2) yields:

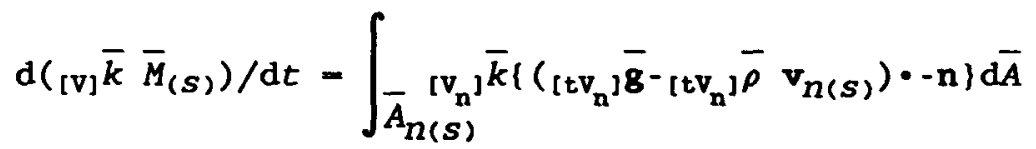

$$
\begin{aligned}
& -\int \bar{A}_{n(s)}\left({ }_{\left[v_{n}\right]} \bar{\mu}+K_{3}[\mathrm{v}] \bar{\mu}^{(t)}\right)\left(\nabla_{\left[v_{n}\right]} \bar{k} \cdot-\mathrm{n}\right) \mathrm{d} \bar{A} \\
& +\bar{V}_{(s)}\left(K_{2}\left([\mathrm{v}] \overline{\mathrm{T}}^{(t)}: \nabla_{[\mathrm{tV}]} \overline{\mathrm{v}}\right)-K_{1[\mathrm{tV}]} \bar{\rho}{ }_{[\mathrm{v}]} \bar{\omega}{ }_{[\mathrm{v}]} \bar{k}\right]
\end{aligned}
$$

and: 


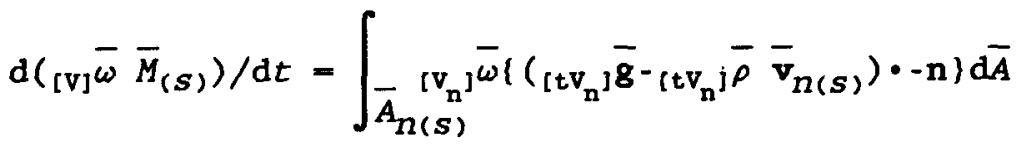

$$
\begin{aligned}
& -\int_{\bar{A}_{n(s)}}\left({ }_{\left[t v_{n}\right]} \bar{\mu}+K_{4}\left[v^{\prime} \bar{\mu}^{(t)}\right)\left(\nabla_{\left[v_{n}\right]} \bar{\omega} \cdot-n\right) d \bar{A}\right. \\
& \left.+K_{5} K_{6} \bar{M}_{(s)}\left[2 l_{[t v]} \bar{D} \cdot\left(\nabla \cdot{ }_{[t v]} \bar{v}-[v] \bar{\omega}\right) I / 3\right\}: \nabla_{[t v]} \bar{v}\right] \\
& -K_{7} \bar{M}_{(s)[\mathrm{v}\}} \bar{\omega}^{2}
\end{aligned}
$$

for the $k$ and $\omega$ transport equations, respectively. Note that the constants $K$ in these integral equations do not correspond exactly to those used in the equations of section 5.6.2.

The remaining equations and constants constituting the implemented version of the $k-\omega$ model will be listed first and discussed thereafter. Two turbulence triggering modes are investigated and are distinguished by referring to them as the 'discontinuous' and 'continuous' modes. The first block of equations and constants are common to both triggering modes and are given by:

$$
\begin{aligned}
& { }_{[\mathrm{V}]} \bar{\mu}^{(\mathrm{t})}=K_{5} K_{10}[\mathrm{tv}]^{\bar{\rho}}[\mathrm{v}]^{\bar{k}} /{ }_{[\mathrm{v}]} \bar{\omega} \\
& K_{5}=\left\{1-\exp \left(-r^{+} / 26.5\right)\right\}^{2} \\
& r^{+}=\left({ }_{[\mathrm{tV}]} \bar{\rho}_{[\mathrm{tv}]} \bar{\tau}_{\mathrm{wa} 11}\right)^{0.5}\left(r_{\mathrm{wa} 11}-r\right) /{ }_{[\mathrm{tV}]} \bar{\mu} \\
& K_{1}=0.09 \quad K_{3}=1 \quad K_{4}=0.5 \\
& K_{6}=\left(3+5 K_{8}\right) / 9 \quad K_{7}=3 / 40 \\
& N_{R e}^{(t)}={ }_{[\mathrm{tV}]} \bar{\rho}[\mathrm{v}] \bar{k} / K_{1}[\mathrm{tv}] \bar{\mu} \quad[\mathrm{V}] \bar{\omega} \\
& {[t v] \bar{D}=0.5\left(\nabla_{[t v]} \bar{v}+\left(\nabla_{[t v]} \bar{v}\right)^{T}\right)}
\end{aligned}
$$

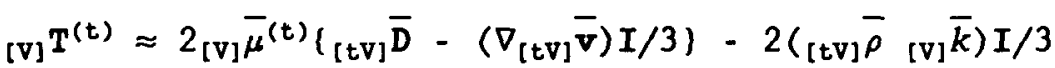


$[\mathrm{v}]^{(\mathrm{t})} \approx K_{1}{ }_{[\mathrm{tV}]} \bar{\rho}_{[\mathrm{v}]}^{\bar{k}}{ }_{[\mathrm{v}]^{\bar{\omega}}} \bar{x}$

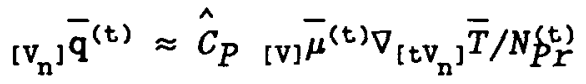

The second block of equations and constants are differentiated by triggering mode as follows:

discontinuous: $K_{8}=5\left[\log \left(1+K_{9}\right)^{\wedge}\left(1+\exp \left(1-K_{9}\right)\right\}\right] / 3$

continuous: $K_{8}=\min \left(\left[5\left[\log \left(1+K_{9}\right)^{\wedge}\left(1+\exp \left(1-K_{9}\right)\right]\right] / 3,1\right)\right.$

discontinuous: $K_{9}=N_{R e}^{(t)} /\left(N_{R e}^{(t)}\right)_{c r i t i c a l}$

continuous: $K_{9}=\left(N_{R e}^{(t)}-1\right) /\left(N_{R e}^{(t)}\right)$ critical

\begin{tabular}{|c|c|}
\hline discontinuous & continuous \\
\hline$\left(N_{R e}^{(t)}\right)_{c r i t i c a l}=486$ & $\left(N_{R e}^{(t)}\right)_{c r i t i c a l}=25$ \\
\hline$K_{2}=1$ & $K_{2}=K_{8}$ \\
\hline$K_{10}=1 \mid\left(N_{R e}\right) \geq\left(N_{R e}\right)_{\text {transition }}$ & $K_{10}=1$ \\
\hline \begin{tabular}{l|l}
$=0$ & $\left(N_{R e}\right)<\left(N_{R e}\right)_{\text {transition }}$
\end{tabular} & \\
\hline
\end{tabular}

Equations (5.22) and (5.23) embrace the foldback function parameters and include enhancements to the Wilcox $k-\omega$ model as discussed below.

a. Near-wall correction methodology

As alluded to previously, the Wilcox $k-\omega$ model includes no near-

wall correction terms. However, in contrast to the coarse radial discretisation typifying the discrete volume analysis used, a much finer spatial discretisation is necessary near the wall to achieve adequate physical accuracy with the basic model. This requirement substantially increases the computational cost of implementing a discrete volume analysis. Inclusion of an appropriate near-wall correction term, however, enables a much lower computational cost to be achieved while retaining scalability. Nagano and Hishida (NH87) investigated a range of existing near-wall correction terms as applied to a $k-\epsilon$ turbulence 
model and proposed an alternative which, they concluded, yields better performance than the existing methods.

Using the $k-\omega$ model, some investigations with both the JonesLaunder (JL72) and Nagano-Hishida near-wall correction methods were performed with the latter method yielding better agreement with the test data at the chosen test point (section 5.5). Hence on this basis, the Nagano-Hishida method was selected pending the availability of further experimental data. The model consists of equations (5.22.2) and (5.22.3) which serve to reduce exponentially the magnitude of the turbulent viscosity as the wall is approached. A dimensionless distance (zero at the wall) defined in terms of the wall shear stress is used as the independent parameter. This ensures that the method is independent of spatial discretisation, so retaining the scalability characteristic of the discrete volume analysis.

b. Constant modifications

Other than the introduction of $K_{5}$ (wall correction term), $K_{2}$ (continuous turbulence trigger parameter) and $K_{6}$ (foldback function parameter), with one exception, the constants are the same as those in the Wilcox $k-\omega$ model. The exception concerns the turbulent diffusion term constant $K_{2}$ in equation (5.14.1) which Wilcox assigned a value of 0.5 (equation $(5.14 .8)$ ) based on numerical experiment. When applied to an oscillating flow, changing this constant to a value of unity $\left(K_{3}\right.$ in equations $(5.20)$ and $(5.22 .4)$ ) yielded better agreement with the experimental data at the test point. Jones and Launder (JL72) and subsequent workers have also used a value of unity for the constant in the $k$ equation turbulent diffusion term (equation (5.11.1)).

\section{c. Turbulence triggering}

As noted in the discussion of equation (5.19), the $k-\omega$ model can only evolve positive turbulent kinetic energy if $k$ is made positive independently. In general, it seems that two approaches to introducing this independence can be considered.

As a first option, the advective triggering mechanism postulated by Seume ( $\mathrm{Se88}$ ) may be used to inject positive $k$ into the twodimensional test section of the METR. This is adequate for the general case provided that such positive $k$ can be generated outside the test section, that is, in the one-dimensional duct between the test section and the heat exchangers. Once a positive $k$ thus has been established, the turbulence evolution may be controlled via a foldback function parameter such as the turbulent Reynolds number (equation (5.22.5)).

A second, more fundamental, option is based on the local acceleration triggering postulate of Hino et al. (HK83). Such a postulate devolves to a triggering model based upon microscopic level entity (molecules or 'clumps' of molecules) dynamics in which random cross-stream or turbulence-inducing momentum arises when the microscopic viscous forces are exceeded by the inertial forces. This mechanism is depicted on a macroscopic level in contained flows by the usual Reynolds number transition criterion. Proceeding further, a microscopic entity 
approach also would allow the discontinuous increase in turbulence observed by Seume (apparently caused by the passage of a turbulent fluid slug) to be naturally modelled so harmoniously blending Hino et al.'s and Seume's observations.

The microscopic turbulence triggering mechanism is considered preferable by the author; however, the project time and budget constraints mitigated against investigation of this concept. Hence, by default, it was decided to proceed with the advective triggering methodology even in the presence of concerns about its physical credibility.

The advective triggering methodology is implemented by creating a pseudo-transport equation for $k$ which is used only in the onedimensional sections of the METR between the test section and the heat exchangers. All turbulence is assumed to be extinguished in the serpentine passages of the heat exchangers. The one-dimensional pseudotransport equation for $k$ is given by:

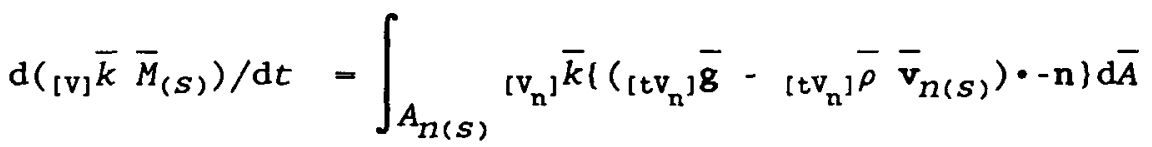

if:

$$
{ }_{[\mathrm{V}]} \bar{k}<{ }_{[\mathrm{tV}]} \overline{\boldsymbol{\tau}}_{\mathrm{wall}} /{ }_{[\mathrm{tV}]} \bar{\rho} \text { and } \quad N_{\operatorname{Re}}>2000
$$

then:

$$
{ }_{[\mathrm{v}]} \bar{k}={ }_{[\mathrm{tv}]} \bar{\tau}_{\mathrm{wa}} /{ }_{[\mathrm{tv}]} \bar{\rho}
$$

Equation (5.24.1) just reflects advective transport of $k$ while equation (5.24.2) initialises $k$ to a floor value of the square of the friction velocity if the Reynolds number is greater than a transition value of 2000. If $k$ is greater than the floor value (irrespective of the Reynolds number) it retains the value computed using equation (5.24.1). This approach yields a fully realizable turbulence triggering method including laminar-to-turbulent transitions from the rest state without the necessity of any $k$ or $\omega$ pre-initialization.

\section{d. Foldback function implementation}

Control of the foldback function is achieved through constants $K_{2}$ and $K_{6}$ (equations $(5.23 .4)$ and $(5.22 .4)$, respectively) together with their threaded substituent constants.

A stability analysis of the $k-\omega$ model showed that the $\omega$ production term in equation (5.21) is an appropriate vehicle for controlling the stability of the foldback function. In particular, the stability boundary hypersurface (Go87) for the model can be intersected for a value of $K_{6}$ between $7 / 9$ and $8 / 9$ (all other constants remaining at their 
listed values). If $K_{6} \leq 7 / 9$, the model exhibits chaos increasing in magnitude until a fully chaotic condition (figure 5.42) is reached for $K_{6} \approx 3 / 9$. For $K_{6} \geq 8 / 9$, the model exhibits 'ordered' behavior (figure 5.39). Thus, continuous variation of $K_{6}$ between $3 / 9$ and $8 / 9$ enables the $k-\omega$ model discontinuously to evolve turbulence in a random fashion.

The continuous variation of $K_{6}$ is achieved via $K_{8}$ (equation (5.23.1)), which varies as an inverse exponential between 0 and 1 , asymptoting to a straight line of small positive slope (0.084) for $K_{9} \geq$ 1. $K_{9}$ is defined as the ratio between the actual turbulent Reynolds number and a 'critical' value determined numerically on the basis of matching the available experimental data.

\section{e. Turbulence triggering modes}

The discontinuous turbulence triggering mode is implemented using the classic Reynolds number transition criterion for pipe flow as shown by equation (5.23.5). In essence, this zeroes $\mu^{(t)}$ for all Reynolds numbers less than the transition number, which is set at 2000 . Note that $K_{2}$ is maintained at unity, thus placing no additional restrictions on the production of $k$ while $\left(N_{R e}^{(t)}\right)_{c r i t i c a l}$ is assigned a relatively high value of 486 , again, based on purely numerical considerations.

The continuous turbulence triggering mode is achieved by allowing $\mu^{(t)}$ to exist regardless of the value of $k$ as long as $\omega>0$ (equation $(5.23 .5))$. However, the foldback function control parameter is modified slightly from its discontinuous form (equation (5.23)) and applied not only to the $\omega$ production term in equation (5.21) but also to the $k$ production term in equation (5.20). Production of $k$ and $\omega$ is thus independent of the bulk flow Reynolds number ( $K_{8}$ has a floor value of unity in its continuous form). ( $\left(N_{R e}^{(t)}\right)_{c r i t i c a l}$ is assigned a value based on the criterion that $k$ is equal to the square of the friction velocity under laminar flow conditions. Physically, this implies a 'continuous' model of fluid turbulence in which all flows contain some level of turbulence except that under laminar conditions, such turbulence is small, stable or 'ordered' and does not increase in the presence of imposed stimuli. Atmospheric flows, for example, are often considered in this light.

Thus, in summary, the $k-\omega$ model is implemented as defined by equations (5.20) to (5.24) at the chosen experimental test point. In view of the foldback function methodology employed, the empirical nature of its constituent parameters, the uncertainty as to which triggering mode is physically accurate and the basic reservations expressed about the advection triggering methodology, this version of the $k-\omega$ model should be viewed as entirely experimental and subject to even drastic revision.

\subsection{COMPARISON CASE EXPERIMENTAL DATA}

The experimental data made available for the comparison case simulation application consists of a half cycle of axial ensemble-averaged mean 
velocities and root-mean-square (RMS) velocity fluctuations at 12 locations on one radial plane. The flow during the half cycle is in the negative direction, that is, from the open end toward the cylinder. The parameters defining the experimental data are listed in table 5.6.

Table 5.6 Parameters for the comparison case experimental data.

\begin{tabular}{lc}
\hline Engine speed: & $33.75 \mathrm{rpm}$ \\
Probe position: & $1676.4 \mathrm{~mm}$ from the cylinder end \\
Dry bulb temperature: & $25.5^{\circ} \mathrm{C}$ \\
Wet bulb temperature: & $15.8^{\circ} \mathrm{C}$ \\
Ambient pressure: & $981 \mathrm{bar}$ \\
No. of cycles for ensemble averaging: & 500 \\
No. of probe radial locations: & 12 \\
\hline
\end{tabular}

Since 500 cycles are used for computing the ensemble averages, in this respect, the data are felt to be adequate for validation purposes. The probe position is located toward the open end of the METR, so enhancing the yield of the comparison and validation process because any experimental flow asymmetries would stress the simulation to a greater extent. Unfortunately, since only negative half-cycle data are available, this aspect of the comparison cannot be accomplished.

Unlike the base case data, no bulk mean velocities were provided for simulation calibration purposes. Hence these velocities are computed from the mean velocities provided using an area-weighted aggregation procedure. The resulting bulk velocity comparisons for the discontinuous and continuous triggering modes (henceforth referred to as the DTM and CTM simulations, respectively) are shown in figures 5.43 and 5.44 . The simulated velocity profiles are identical showing that the triggering mode has no influence on the bulk velocities, as expected from a conservative set of transport equations. The experimental data reveal a small phase shift relative to the simulated data which is apparent at $0^{\circ}$ and $180^{\circ}$. However, of major concern are the large amplitude, higher order harmonic fluctuations in the experimental data between $60^{\circ}$ and $120^{\circ}$. Apparently, these fluctuations are related to piston/cylinder stiction and/or sealing effects. Nevertheless, their magnitude and shape has a major impact on the behavior of the flow turbulence, so complicating the comparison of the experimental and simulated $k$ between $60^{\circ}$ and $120^{\circ}$ because the simulated $k$ is not subject to the same production stimuli as the experimental $k$. Clearly, these fluctuations in the mean experimental data should be removed before any further comparison or validation work is undertaken.

\subsection{COMPARISON CASE RESULTS}

An overview of the cyclic energy balances for the DTM and CTM simulation runs is shown in table 5.7. 


\section{SIMULATED AND EXPERIMENTAL BULK VELOCITY PROFILES}

Probe at $1676 \mathrm{~mm}$

Reynolds no: 11706

Valensi no: 79.6

simulation

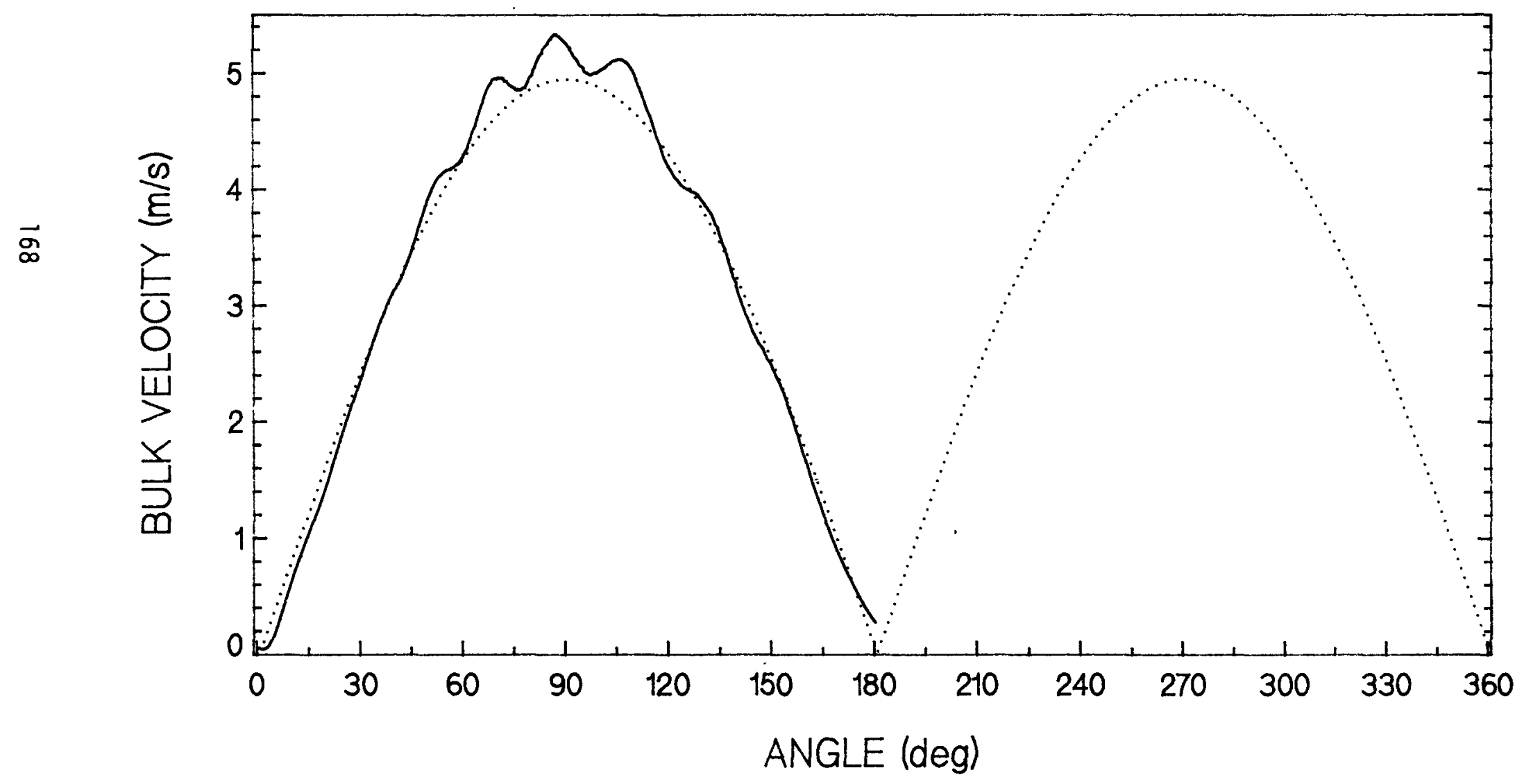

Figure 5.43 Discontinuous triggering mode 


\section{SIMULATED AND EXPERIMENTAL BULK VELOCITY PROFILES}

Probe at $1676 \mathrm{~mm}$ Reynolds no: 11706
Valensi no: 79.6

simulation

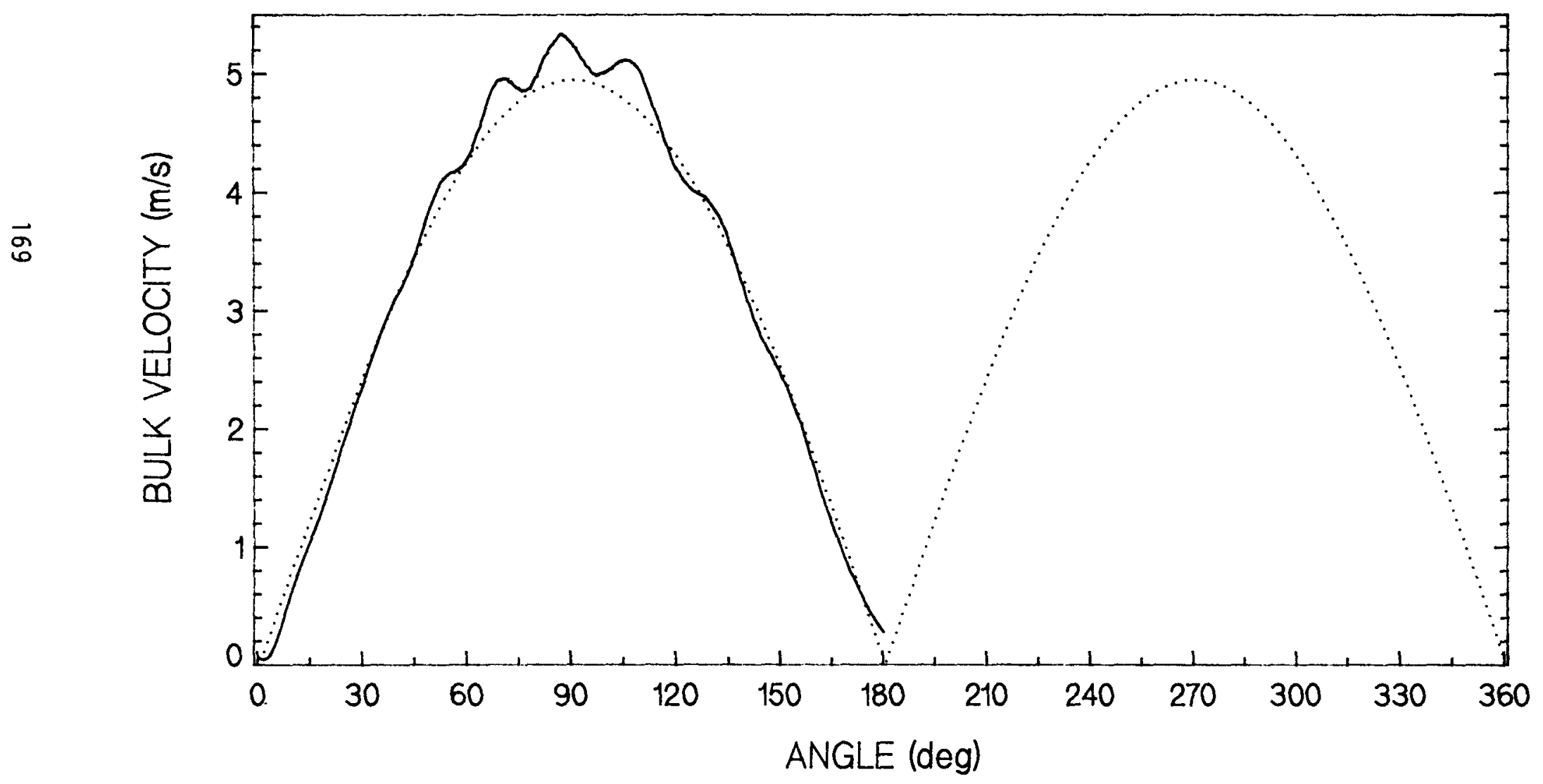

Figure 5.44 Continuous triggering mode 
Table 5.7 Comparison case cyclic energy balances.

\begin{tabular}{lccccc}
\hline Triggering mode & $\begin{array}{c}\text { Net Heat } \\
\text { Input } \\
(\mathrm{J})\end{array}$ & $\begin{array}{c}\text { Net Heat } \\
\text { Output } \\
(\mathrm{J})\end{array}$ & $\begin{array}{c}\text { Net Kinetic } \\
\text { Energy Con- } \\
\text { version }(\mathrm{J})\end{array}$ & $\begin{array}{c}\text { Indicated } \\
\text { Work Done } \\
(\mathrm{J})\end{array}$ & $\begin{array}{c}\text { Energy } \\
\text { Balance } \\
\text { Error }(8)\end{array}$ \\
\hline discontinuous & 0.2482 & 0.7023 & 0.2847 & -0.1693 & 0.01016 \\
continuous & 0.223 & 0.4347 & 0.0578 & -0.1538 & 0.015098 \\
\hline
\end{tabular}

The energy balance errors reported were realised after three simulated cycles. of significance is that the net kinetic energy conversion for the DTM simulation is four times greater than that of the CTM simulation. This is attributable to the turbulence magnitude being larger in the DTM simulation (see figures 5.55 and 5.56 ).

The mean velocity profiles at the test section are compared in figures 5.45 to 5.47. The fluctuations apparent in figures 5.43 and 5.44 are also evident in figure 5.45 and are manifest through the boundary layer. The magnitude of the $12.5 \%$ discrepancy in the simulated and experimental maximum mean velocities is mainly attributable to the fluctuations in the experimental mean velocities. Although figures 5.46 and 5.47 are similar, the DTM simulation appears to correspond more closely to the experimental data over the first quarter of the cycle than the CTM simulation. This is confirmed by figures 5.48 and 5.49 , particularly below $50^{\circ}$. As noted above, because the difference in core velocities is largely due ta systematic experimental effects which are not included in the simulation, the impact of turbulence model errors on the mean velocity profiles cannot be readily determined. Hence, ignoring the magnitude discrepancies, figure 5.50 shows that the CTM simulation yields velocity profiles that parallel their experimental counterparts fairly well in the core flow region. However, the simulated velocity profiles in the boundary layer do not agree well with the experimental data beyond $116.5^{\circ}$. Returning to figures 5.48 and 5.49 , the DTM simulation seems to yield a closer correspondence to the experimental data in the boundary layer than the CTM simulation. Comparing figures 5.48 to 5.50 with figure 5.35 (showing data produced without a turbulence model), it is evident that inclusion of a turbulence model does yield significantly better correspondence between the simulated and experimental data in the core flow region.

The discrepancies between the experimental and simulated boundary layer mean velocity profiles most likely are attributable to the turbulence triggering mechanism used in the simulation. The experimental axial RMS velocity fluctuations are shown in figure 5.51. These fluctuations are converted into turbulent kinetic energy $(k)$ in figure 5.52. Strictly, this $k$ cannot be compared with that simulated because the simulated $k$ includes both axial and radial components while the measured $k$ consists of an axial component only. The $k-\omega$ and $k-\epsilon$ models assume that $k$ is isotropic (that is, the axial and radial components are equal) which may not be true in oscillating flows. However, without experimental radial velocity fluctuation data, no further determination on this issue can be made here. 


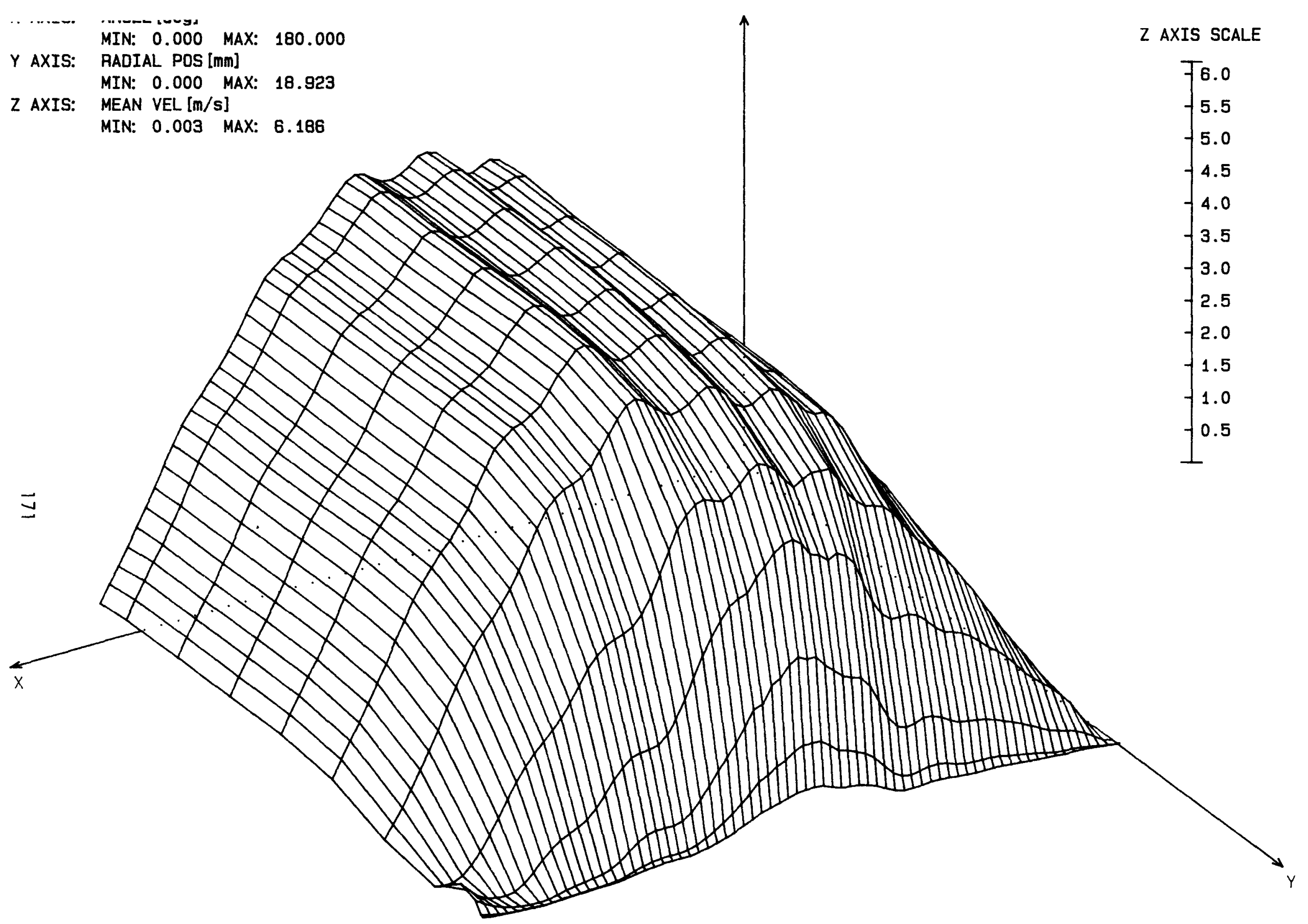

FIGURE 5.45 EXPERIMENTAL NEGATIVE HALF CYCLE DATA $(R e=11706 ; \quad V a=79.6)$ 


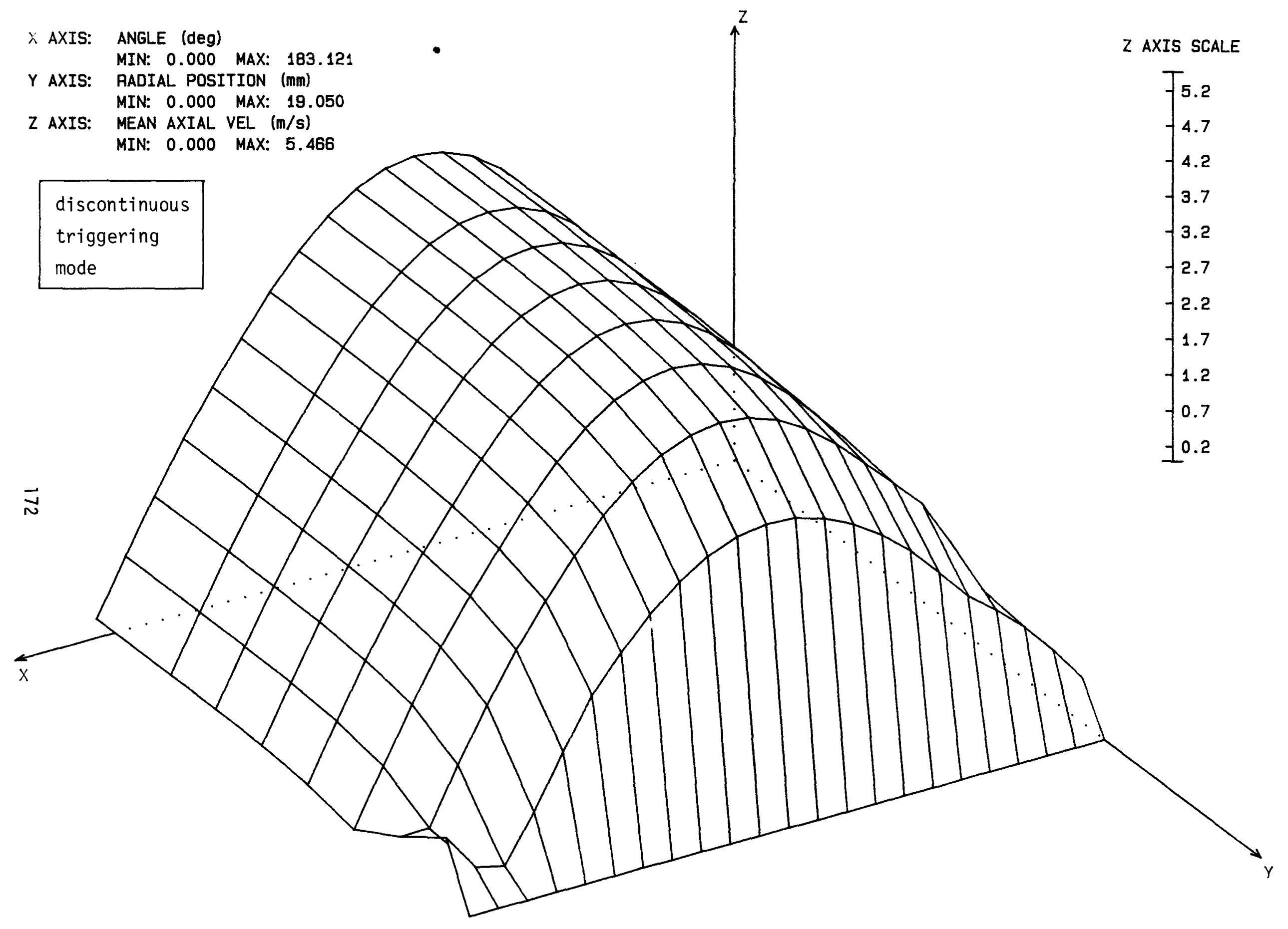

FIGURE 5.46 SIMULATION NEGATIVE HALF CYCLE DATA $(R e=11706 ; \quad V a=79.6)$ 


\section{RADIAL PROFILES OF MEAN AXIAL VELOCITY}

Probe at $1676 \mathrm{~mm}$ Reynolds no: 11706 Valensi no: 79.6

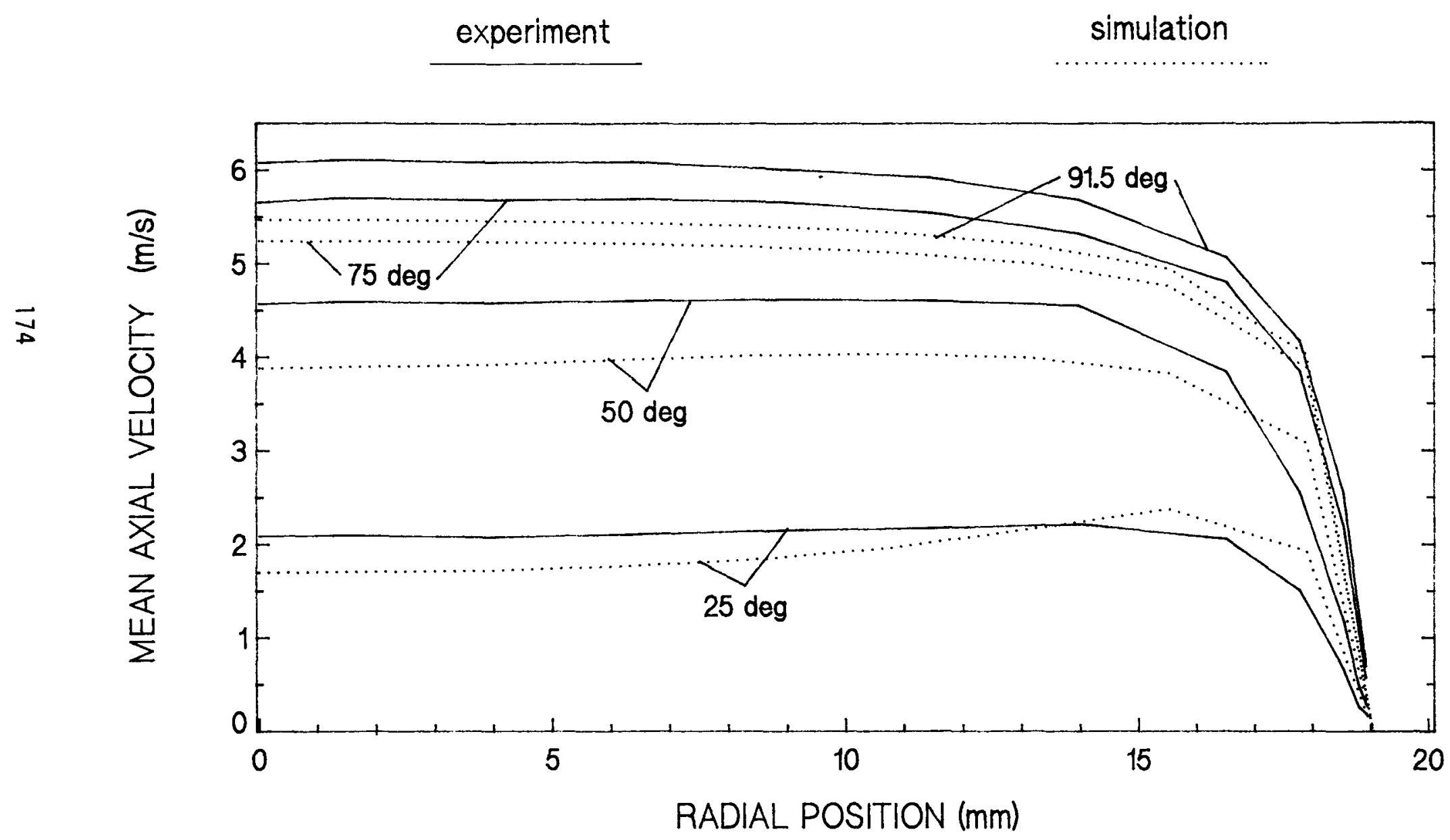




\section{RADIAL PROFILES OF MEAN AXIAL VELOCITY}

Probe at $1676 \mathrm{~mm} \quad$ Reynolds no: 11706 Valensi no: 79.6

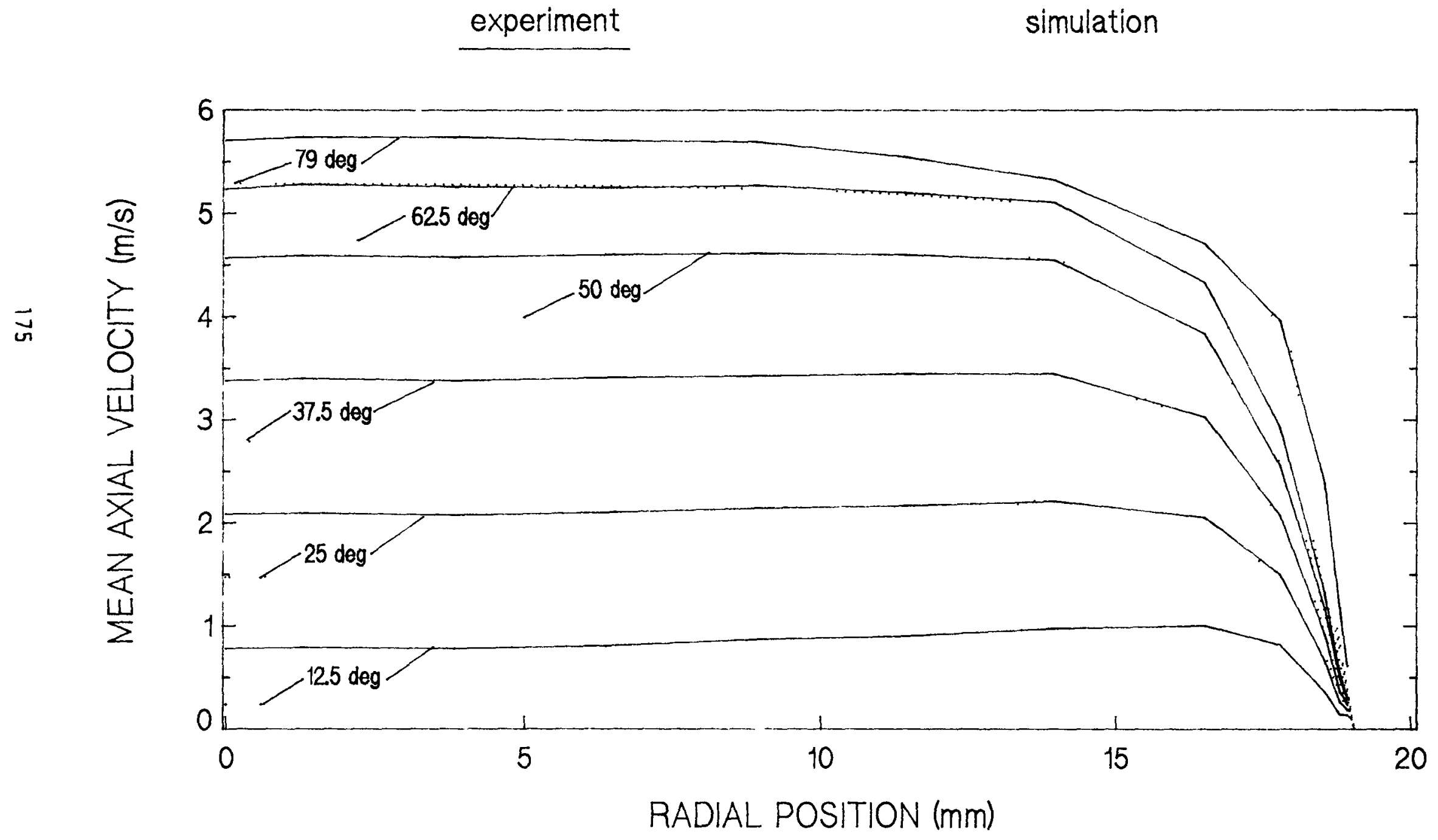

Figure 5.49 Continuous triggering mode 


\section{RADIAL PROFILES OF MEAN AXIAL VELOCITY}

Probe at $1676 \mathrm{~mm}$

Reynolds no: 11706

Valensi no: 79.6

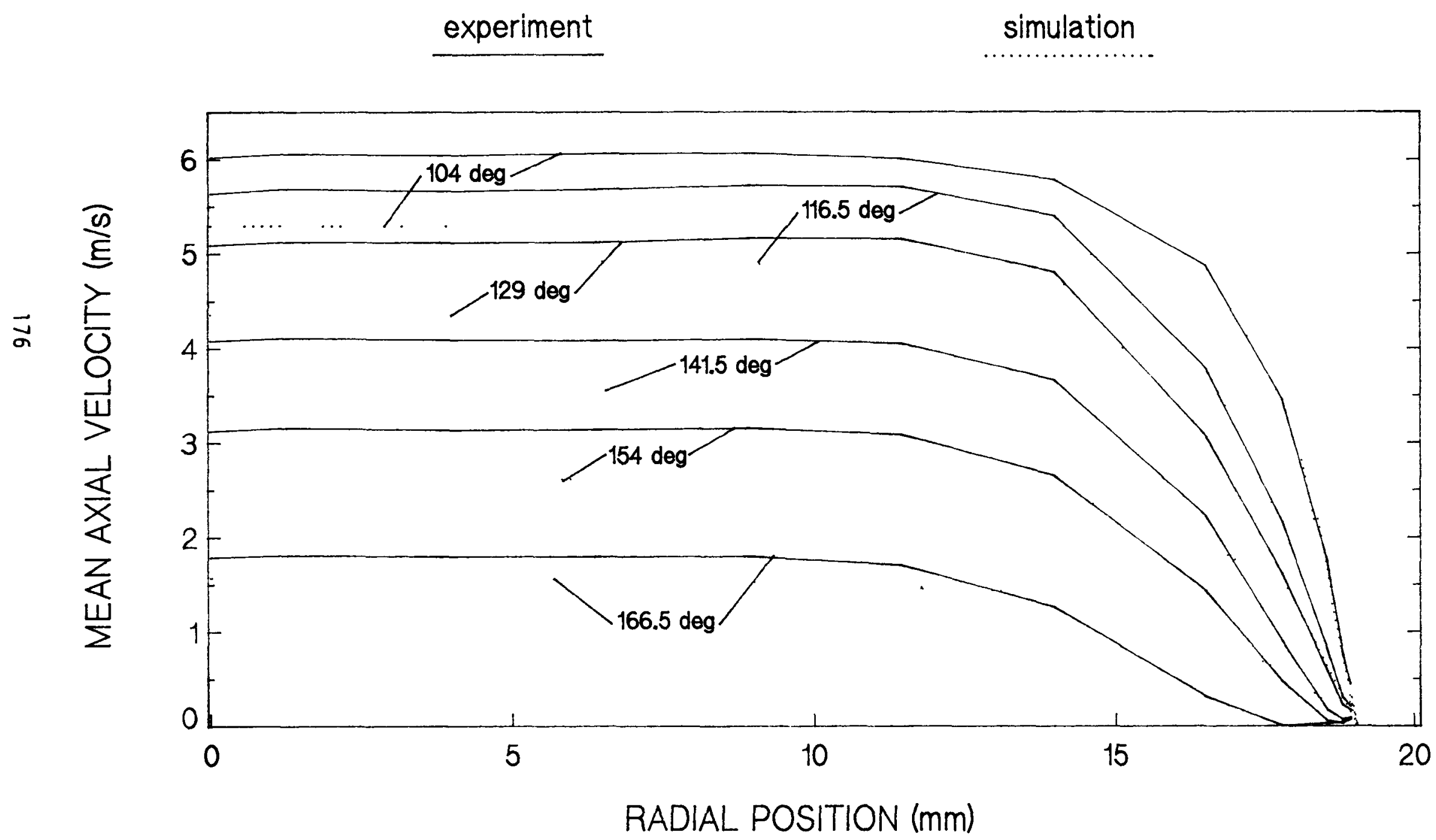

Fiaure 550 Continunus triaaerina mode 


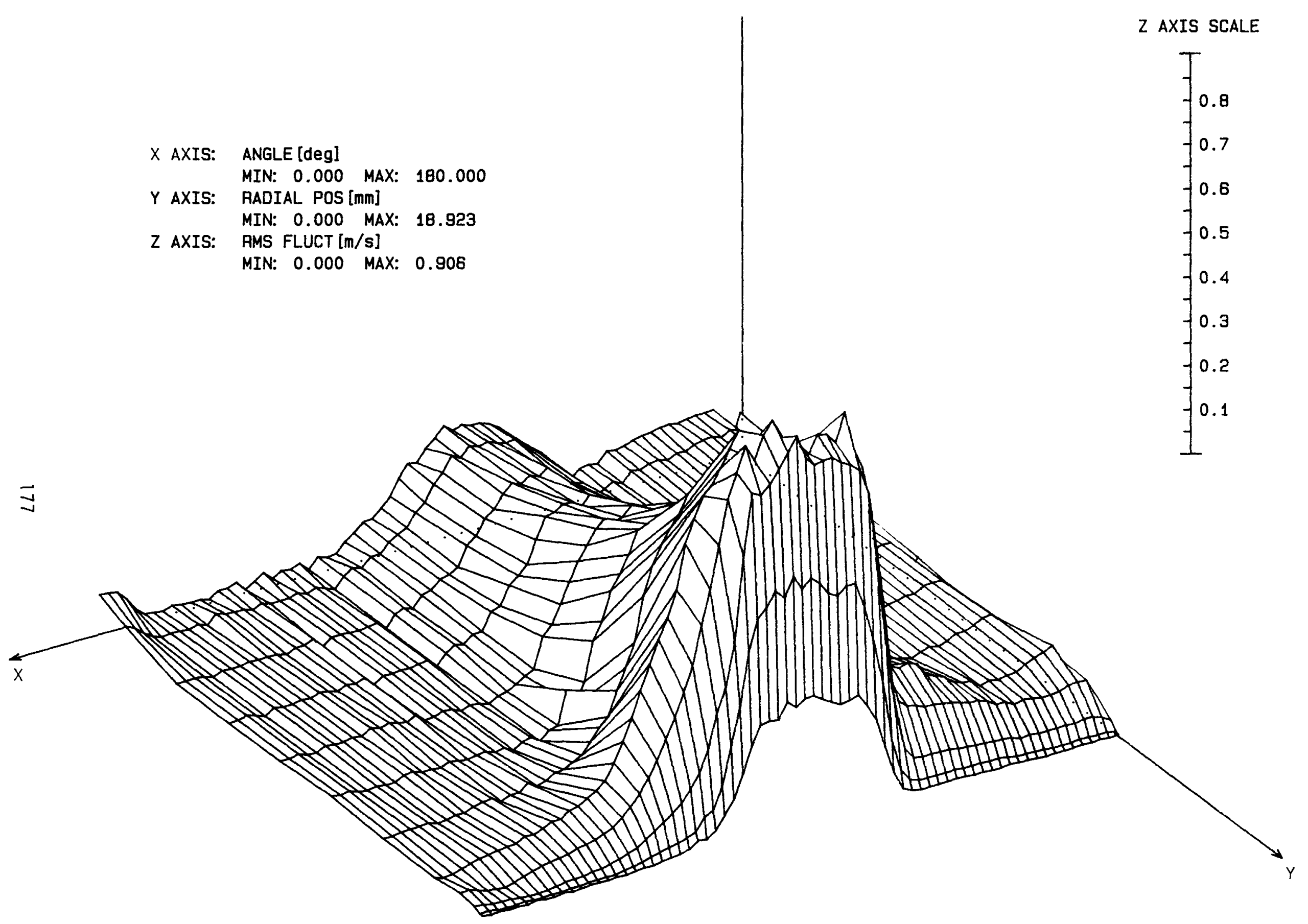

FIGURE 5.51 EXPERIMENTAL NEGATIVE HALF CYCLE DATA $(R e=11706 ; \quad V a=79.6)$ 


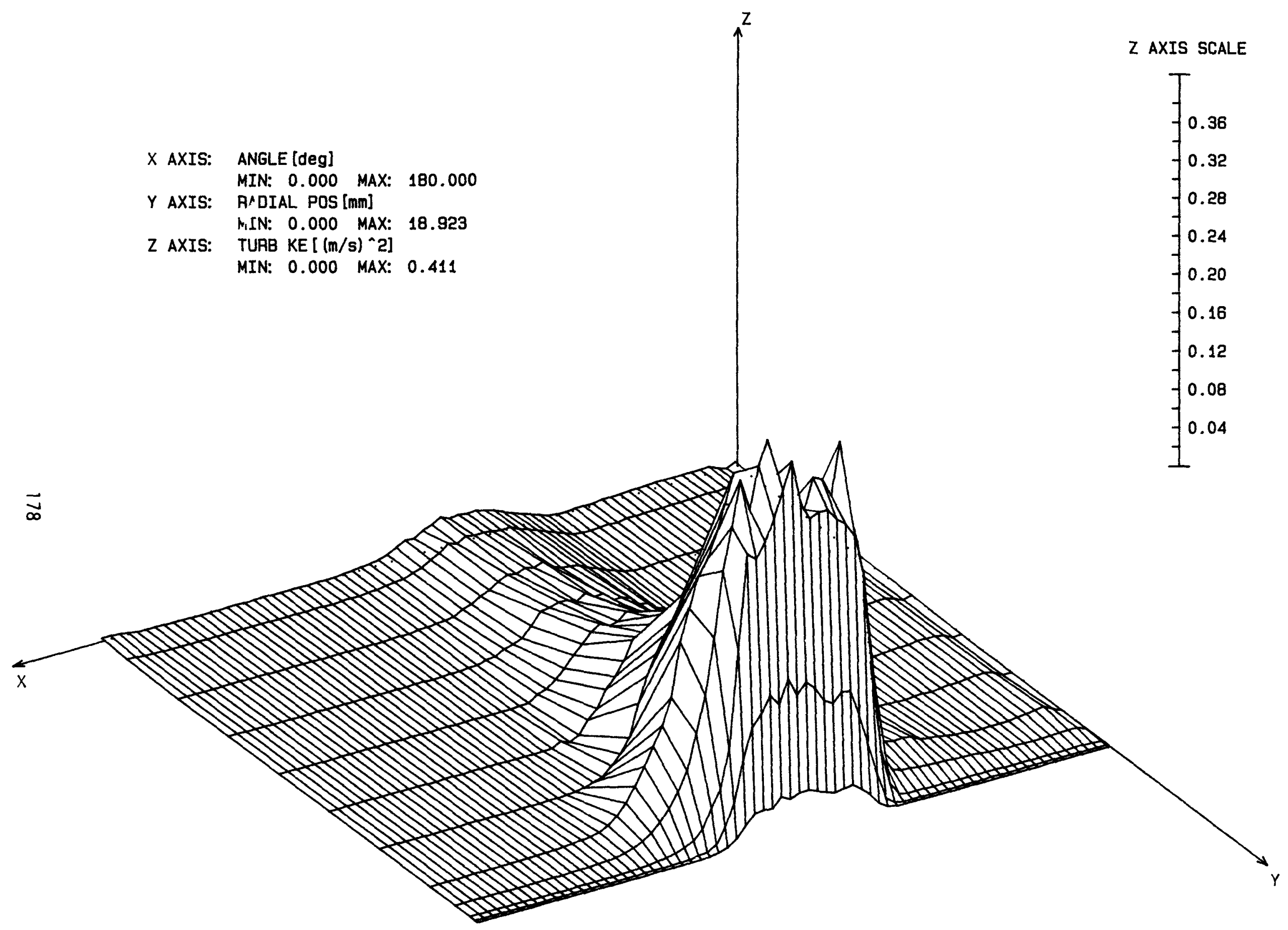

FTRIIRF 55) FXPFRTMFNTAL NEGATIVE HALF CYCLE DATA (Re=11706; Va=79.6) 
With this reservation, the experimental and simulated turbulent kinetic energies are compared in figures 5.52 to 5.54 . The effects of the experimental mean velocity fluctuations on the turbulent kinetic energy is readily apparent in figure 5.52, which thus does not display the same smoothness as figures 5.53 and 5.54 . The overall shape of the DTM simulation $k$ surface corresponds better to the experimental data than that of the CTM simulation (figure 5.54). However, the DTM simulation turbulence triggering occurs too early compared with the experimental observations, that is, the discontinuous triggering level (based on bulk flow considerations) of $\mathrm{N}_{R e}=$ 2000 is too low. It may thus be inferred that discontinuous triggering is physically more accurate but that a bulk flow parameter such as the Reynolds number is inadequate as a trigger parameter. As suggested in section $5.6 .3(\mathrm{c})$, use of a microscopically based trigger parameter may prove to be physically more realistic.

The CTM simulation depicts the existence of relatively large $k$ over the entire half cycle (figure 5.54) and thus does not correspond to the physical behavior depicted in figure 5.52. In contrast, the DTM simulation yields a maximum $k 3.8$ times greater than that measured while the CTM simulation and measured $k$ are within 208 of each other. Assuming that the isotropic turbulence assumption is valid for the experimental flow, then a net maximum experimental $k$ of $.811(\mathrm{~m} / \mathrm{s})^{2}$ falls one-third of the way between the CTM and DTM simulated values. This warrants a suspicion that the CTM simulation is more accurate in predicting the evolution of turbulence once it has been triggered while the DTM simulation better reflects the triggering mechanism itself. Lastly, the differences in turbulence triggering revealed in figures 5.52 to 5.54 can be seen to account for the discrepancy in the mean velocity profiles evident in figures 5.48 to 5.50 .

The full-cycle DTM and CTM simulations produce radically different surfaces as shown in figures 5.55 and 5.56. As there is no experimental data against which the simulated full-cycle $k$ data may be directly compared, qualitative recourse again must be made to figure 5.38 , which is the closest data point in Seume's thesis to the comparison case test point. Based on a comparison of figures $5.38,5.55$ and 5.56 , it is apparent that, topologically, the DTM simulation produces far better correspondence with the experimental data than the CTM simulation. Examination of figure 5.55 reveals that the magnitude of the negative half-cycle turbulence $\left(0^{\circ}\right.$ to $\left.180^{\circ}\right)$ is slightly less than the positive half-cycle turbulence, thus mimicking the trend of figure 5.38. However, the simulated relative positive and negative half-cycle kinetic energies are probably in error. Figure 5.56 bears little resemblance to figure 5.38 revealing inverted behavior, that is, negative half-cycle $k$ greater than the positive half-cycle $k$. Based on this comparison, it must be concluded that continuous triggering does not appear to be physically viable, at least for the two-parameter, $k-\omega$ model evaluated. 


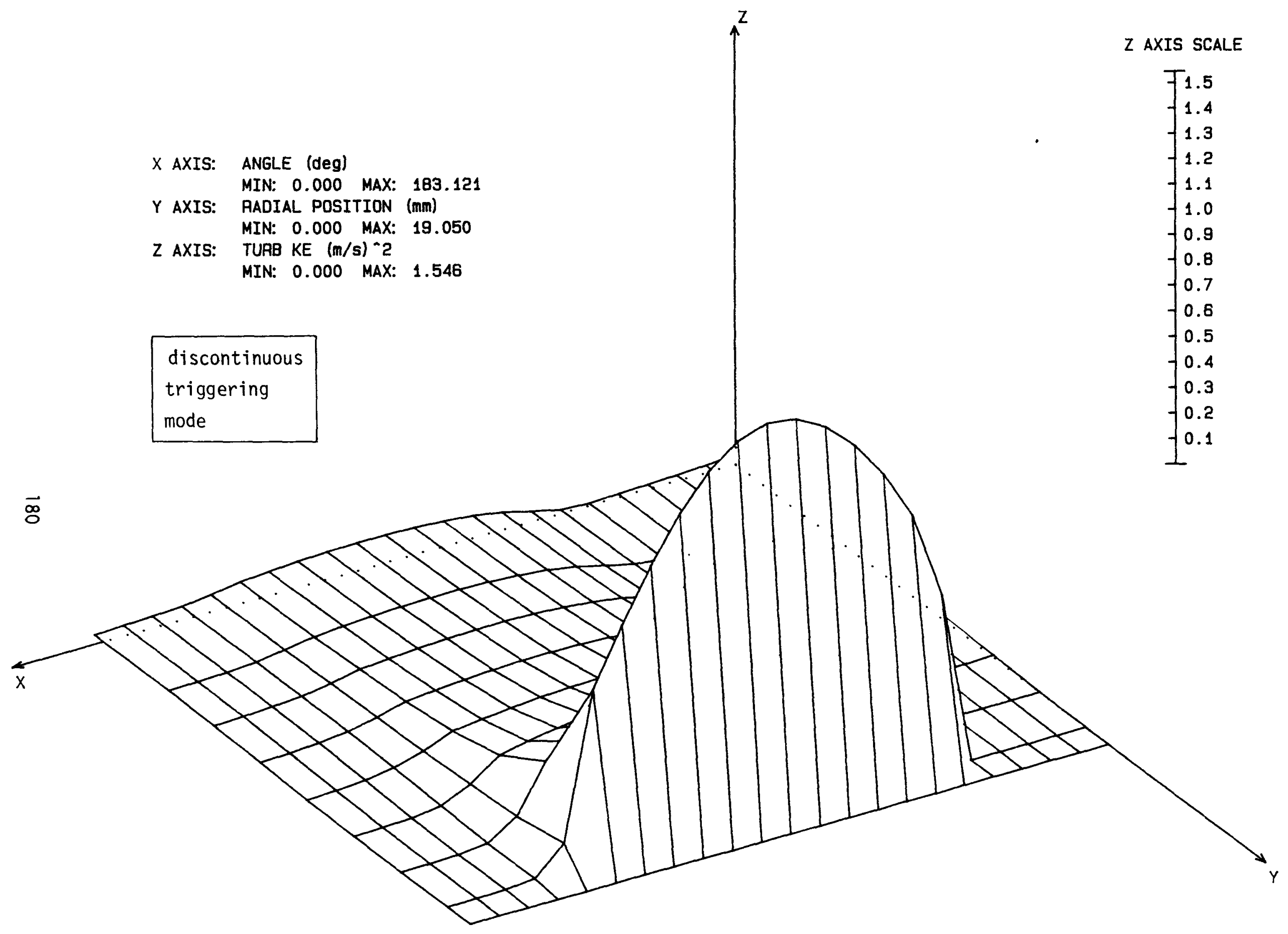

FIGURE 5.53 SIMULATION NEGATIVE HALF CYCLE DATA (Re=11706; Va=79.6) 


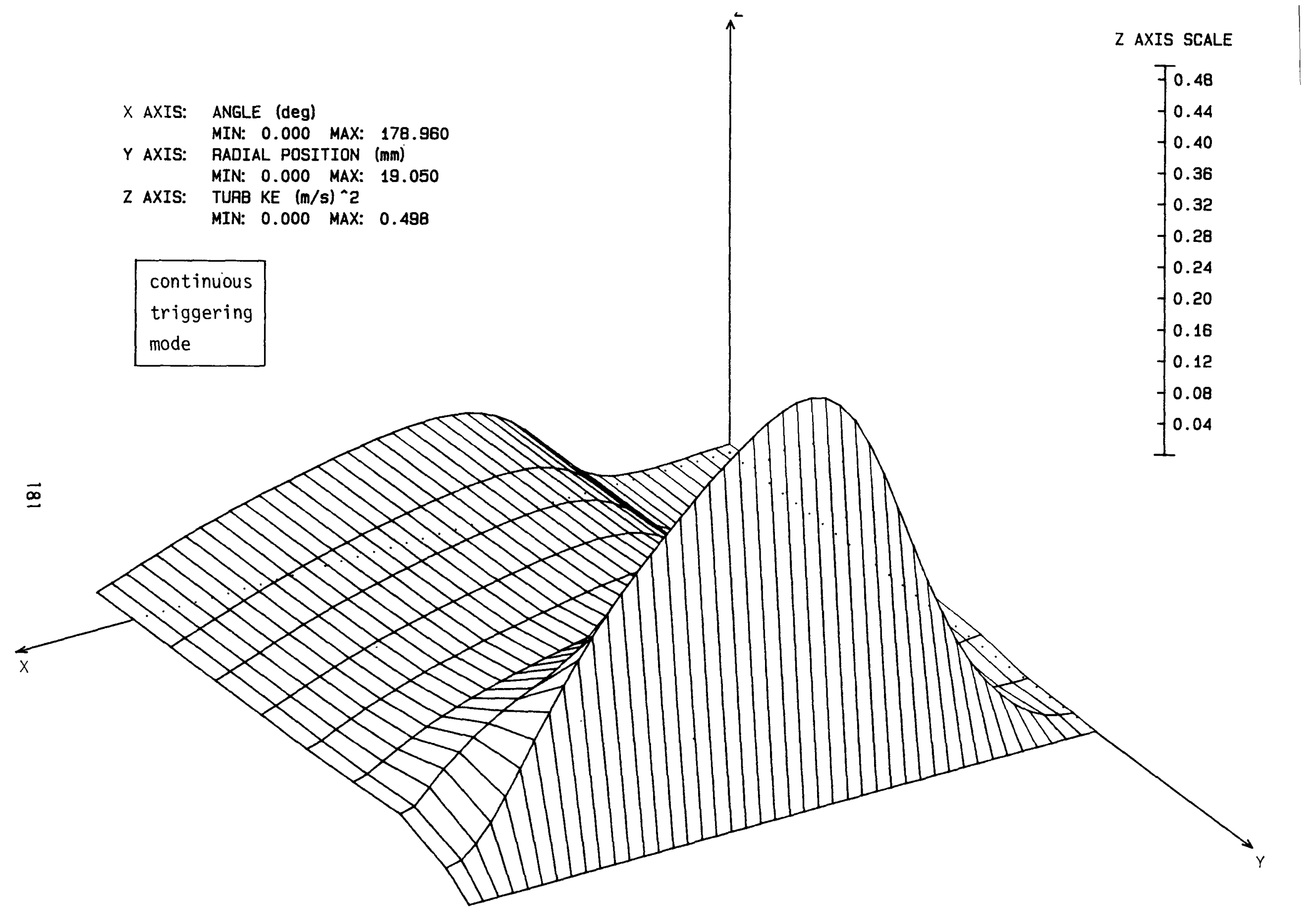

FIGURE 5.54 SIMULATION NEGATIVE HALF CYCLE DATA (Re=11706; Va=79.6) 


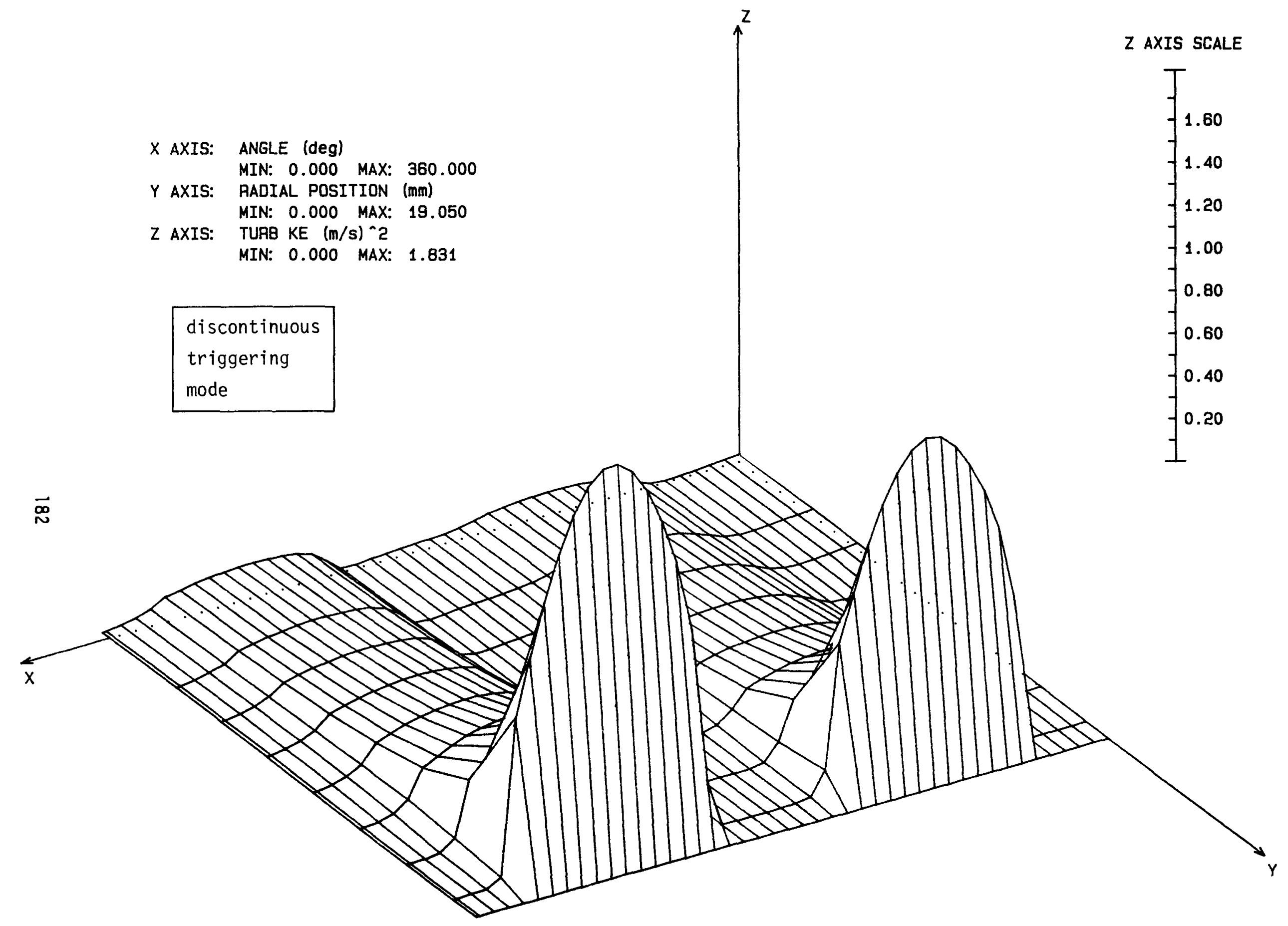

FIGURE 5.55 SIMULATION FULL CYCLE DATA (Re=11706; Va=79.6) 


\subsection{CONCLUSION}

In the context of the limited experimental data available, the results show that a two-parameter, $k-\omega$ foldback function turbulence model incorporating a discontinuous triggering mode is capable of producing qualitatively reasonable turbulence predictions. In terms of the mean flow parameter predictions, which are important to stirling machine design and analysis, two-parameter turbulence models of the $k-\omega$ type may be adequate for most purposes, so making use of a more sophisticated (although rigorously preferable) Reynolds stress turbulence model unnecessary.

Viewing the simulation and testing of the METR as a whole, it seems that the advective turbulence triggering hypothesis of Seume is the predominant triggering mechanism, while the local acceleration has more influence on controlling the evolution of the turbulence once triggering has occurred. This triggering mechanism is fundamentally discontinuous, requiring the development of a turbulence triggering parameter which is fully realizable in terms of the turbulence model variables. The bulk flow Reynolds number does not appear to be suitable because it is macroscopically rather than microscopically defined. The experimental and simulated data both suggest that a microscopically based triggering parameter is required for physical accuracy.

The turbulence model development and testing reported should be viewed only as an exploratory first step that has highlighted the turbulence triggering issue as being cardinal to the successful simulation of oscillating turbulent flows. More experimental data and simulation development is necessary before the $k-\omega$ turbulence model presented is suitable for application to the oscillating flows found in Stirling cycle machines. 


\section{CHAPTER 6}

$\begin{array}{llllllllll}C & 0 & N & C & L & U & S & I & O & N\end{array}$

Three of the issues that have emerged during the course of this project as being of significance to Stirling machine simulation are:

1. Two-dimensional component simulation of Stirling machines does not necessarily yield any improvement over one-dimensional simulation in overall performance prediction.

2. The ability to model information propagation effects at low Mach numbers may be a necessary attribute for Stirling machine simulation codes. This determination may be made in terms of the characteristic number $\left(N_{c h}\right)$ which appears to be a useful additional non-dimensional parameter for describing Stirling machine oscillating flows.

3. Turbulence triggering is a key element in implementing a successful turbulence model for oscillating flows.

While simulating the METR turbulent flow field, the need for reliable oscillating turbulent flow experimental data spanning a broad range of boundary conditions has become apparent. As advective boundary conditions have a major, if not overriding, significance on the turbulence triggering mechanism in oscillating flows, particular care should be taken to quantify these boundary conditions completely. However, it must be recognised that turbulence models validated using experimental data generated with nonStirling advective boundary conditions may not be immutably applicable to Stirling machines themselves because of the inherently chaotic nature of the triggering process. Thus, ultimately, such turbulence models may have to be validated against turbulent flow data gathered in Stirling machines directly.

Although two-dimensional component simulation is clearly of value in understanding the flow details of Stirling machines, from the work carried out here, it is not yet evident that such component simulation provides any better prediction of overall machine performance than one-dimensional simulation. From a design perspective, two-dimensional component simulation thus may serve best as a means of generating improved turbulence correlations for use in existing one-dimensional codes. Nevertheless, the continuing evolution of computer hardware will eventually make end-to-end, two-dimensional simulation of Stirling machines a cost-effective reality.

Use of qualifying benchmark tests for stirling machine simulation codes may prove to be an effective means of ensuring that these codes meet a minimum standard of validity, particularly if they are used to design novel (and expensive) Stirling hardware. Iberall's (Ib50) experimentally validated 
(Wa65, Go68) analytic solution of the transmission line problem is an ideal candidate for a qualifying benchmark test.

As a general proposition, numerical methods are an important facet of Stirling machine simulation to the extent that the numerical method itself emulates a physical process. Thus, simulation errors may be a result of the physical implications of a particular numerical method, rather than just its numerical accuracy implications. In this light, an isolated investigation of pressure-1inking as a legitimate numerical algorithm for simulating low characteristic number compressible flows is warranted. 
BB79 Book, D.L., Boris, J.P., and Hain, K. "Flux-corrected transport II: generalizations of the method," Journal of Computational Physics; vol. 18, 1975, pp. 248-283.

Be78 Berchowitz, D.M. A Computer and Experimental Simulation of Stirling Cycle Machines, MSc dissertation, University of the Witwatersrand, Johannesburg, 1978.

Br87 Brown, A.T. SPDE Phase I Final Report, NASA contractor report no. 179555 (MTI 87TR36), 1987.

BS60 Bird, R.B., Stewart, W.E., and Lightfoot, E.N. Transport Phenomena, John Wiley \& Sons, New York, 1960.

CF67 Courant, R., Friedrichs, K., and Lewy, H. "On the partial difference equations of mathematical physics," IBM Journal, vol. $11,1967, \mathrm{pp} .215-234$.

Ch64 Chester, W. "Resonant oscillations in closed tubes," Journal of Fluid Mechanics, vol. 18, part 1, 1964, pp. 44-46.

Fe83 Ferziger, J.H. "Higer-level simulations of turbulent flows," Computational Methods for Turbulent, Transonic and Viscous Flows, Essers, J.A., (ed.), Hemisphere, Washington, D.C., 1983.

Ge76 Geisow, A.D. The Onset of Oscillations in a Lossless Fluidyne, UK Atomic Energy Authority, Harwel1, report no. AERE-M2840, 1976.

GE76 Gessner, F.B., and Emery, A.F. "A Reynolds stress model for turbulent corner flows -- part I: development of the mode1," Journal of Fluids Engineering, pp. 261-268, June 1976.

Ge86 Gedeon, D. "A globally implicit Stirling cycle simulation," Proceedings of the 21st IECEC, paper no. 869121, pp. 550-554, 1986.

GM66 Gentry, R.A., Martin, R.E., and Daly, B.J. "A Eulerian differencing method for unsteady compressible flow problems," Journal of Computational Physics, vol. 1, 1966, pp. 87-118.

Go68 Goldschmeid, F.R. On the Dynamic Performance of Viscous Compressible Fluids in Rigid Tubes with Volume Termination as a Function of the Stokes Number, NASA TM X-53785, 1968.

Go87 Goldberg, L.F. A State Space and Continuum Mechanics Analysis of Stirling Cycle Machines, PhD thesis, University of the Witwatersrand, Johannesburg, 1987. 
HA71 Harlow, F.H., and Amsden, A.A. "A numerical fluid calculation method for all flow speeds," Journal of Computational Physics, vol. 8, 1971, pp. 197-213.

HF64 Harlow, F.H., and Fromm, J.E. "Dynamics and heat transfer in the von Karman wake of a rectangular cylinder," The Physics of Fluids, vol. 7, no. 8, 1964, pp. 1147-1156.

Hi75 Hinze, J.0. Turbulence, 2nd edition, McGraw-Hill, New York, 1975.

HK83 Hino, M., Kashiwayanagi, M., Nakayama, A., and Hasa, T.

"Experiments on the turbulence statistics and the structure of a reciprocating oscillatory flow," Journal of Fluid Mechanics, vol. 131, pp. 363-400, 1983.

HL72 Hanjalic, K., and Launder, B.E. "A Reynolds stress model of turbulence and its application to thin shear flows," Journal of Fluid Mechanics, vol. 52, part 4, pp. 609-638, 1972.

HW65 Harlow, F.H., and Welch, J.E. "Numerical Calculation of TimeDependent Viscous Incompressible Flow of Fluid with Free Surface," The Physics of Fluids, vol. 8, no. 12, 1965, pp. 2182-2189.

Ib50 Iberall, A.S. Attenuation of Oscillatory Pressures in Instrument Lines, research paper RP2115, vo1. 45, NBS, 1950.

Ji73 Jimenez, J. "Nonlinear gas oscillations in pipes. Part I. Theory," Journal of Fluid Mechanics, vol. 59, part 1, 1973, pp. $23-46$.

JL72 Jones, W.P., and Launder, B.E. "The prediction of laminarization with a two-equation model of turbulence," International Journal of Heat and Mass Transfer, vol. 15, pp. 301-314, 1972.

Jo69 John, J.E.A. Gas Dynamics, Allyn and Bacon, Boston, 1969.

KL64 Kays, W.M., and London, A.L. Compact Heat Exchangers, 2nd edition, McGraw-Hil1, New York, 1964.

LR75 Launder, B.E., Reece, G.J., and Rodi, W. "Progress in the development of a Reynolds-stress turbulence closure," Journal of Fluid Mechanics, vo1. 68, part 3, pp. 537-566, 1975.

LS74 Launder, B.E., and Spalding, D.B. "The numerical computation of turbulent flows," Computer Methods in Applied Mechanics and Engineering, vol. 3, pp. 269-289, 1974.

Lu78 Lumley, J.L. "Computational modelling of turbulent flows," Advances in Applied Mechanics, vol. 18, p. 123, 1978. 
Mc82 MacCormack, R.W. "A numerical method for solving the equations of compressible viscous flow," AIAA Journal, vol. 20, no. 9, pp. $1275-1281,1982$.

NH87 Nagano, Y., and Hishida, M. "Improved form of the $k-\epsilon$ model for wall turbulent shear flows," Journal of Fluids Engineering, vol. 109, pp. 156-160, June, 1987.

Or82 Organ, A.J. "Gas dynamics of the temperature determined Stirling cycle," Journal of Mechanical Engineering Science, vol. 23, no. 4, 1982, pp. 207-216.

RK49 Redlich, O., and Kwong, J.N.S. "On the thermodynamics of solutions. V," Chemical Reviews, vo1. 44, no. 1, 1949, pp. 233244.

Ro82 Roache, P.J. Computational Fluid Mechanics, Hermosa Publishers, Albuquerque, 1982 .

Ro84 Rodi, W. Turbulence Models and Their Application in Hydraulics -. A State of the Art Review, Institute für Hydromechanik, University of Karlsruhe, Karlsruhe, 1984.

$\operatorname{Sc} 1871$

Schmidt, G. "Theorie der Lehmann'schen calorischen maschine," Zeitschrift des Vereines Deutscher Ingenieure, vol. 15, part 1, Jan. 1871, pp. 1-12, part 2, Feb. 1871, pp. $97-112$.

Sc78 Schock, A. "Stirling engine analysis program," AIAA Journal of Energy, vol. 2, no. 6, Nov.-Dec. 1978, pp. 354-362.

Sc79 Schlichting, H. Boundary-Layer Theory, 7th edition, McGraw-Hill, New York, 1979.

Sc83 Schreiber, J. Testing and Performance Characteristics of a $1-\mathrm{kW}$ Free Piston Stirling Engine, NASA Technical Memorandum 82999, 1983 .

Se88 Seume, J.R. An Experimental Investigation of Transition in Oscillating Pipe Flow, PhD thesis, University of Minnesota, Minneapolis, 1988 .

S181 Slattery, J.C. Momentum, Energy and Mass Transfer in Continua, 2nd edition, Krieger, New York, 1981.

Sp72 Spalding, D.B. The $k-w$ Model of Turbulence, Imperial College, Mech. Eng. Departmental Report TM/TN/A/16, 1972.

Sp87 Speziale, C.G. "On nonlinear $k-1$ and $k-\epsilon$ models of turbulence," Journal of Fluid Mechanics, vol. 178, pp. 459-475, 1987. 
St26 Strutt, J.W. (Lord Rayleigh) The Theory of Sound (Vo1. II), 2nd edition, Macmillan, London, 1926.

Ta84 Taylor, D.R. "The method of characteristics applied to Stirling engines," Proceedings of the 19th IECEC, paper no. 849177, San Francisco, 1984.

Te83 Tew, R.C., Jr. Computer Program for Stirling Engine Performance Calculations, NASA TM-82960, DOE/NASA/51040-42, 1983.

Te88 Tew, R. Personal communication, 1988.

Th79 Thieme, L.G. Low-Power Baseline Test Results for the GPU 3 Stirling Engine, NASA Technical Memorandum 79103, 1979.

Ti66 Tisza, L. Generalized Thermodynamics, MIT Press, Cambridge, 1966.

TL72 Tennekes, H., and Lumley, J.L. A First Course in Turbulence, MIT Press, Cambridge, 1972 .

TM72 Taylor, A.E., and Mann, W.R. Advanced Calculus, 2nd edition, John Wiley and Sons, New York, 1972.

Ur77 Urieli, I. A Computer Simulation of Stirling Cycle Machines, PhD thesis, University of the Witwatersrand, Johannesburg, 1977.

Wa65 Watts, G.P. An Experimental Verification of a Computer Program for the Calculation of Oscillatory Pressure Attenuation in Pneumatic Transmission Lines, report no. LA-3199-MS, Los Alamos Scientific Laboratory, 1965.

Wa73 Walker, G. Stirling-Cycle Machines, Clarendon Oxford University Press, 1973.

Wi88 Wilcox, D.C. "Reassessment of the scale-determining equation for advanced turbulence models," AIAA Journal, vol. 26, no. 11, pp. $1299-1310,1988$.

ZH76 Zucrow, M.J., and Hoffman, J.D. Gas Dynamics, John Wiley and Sons, New York, 1976. 


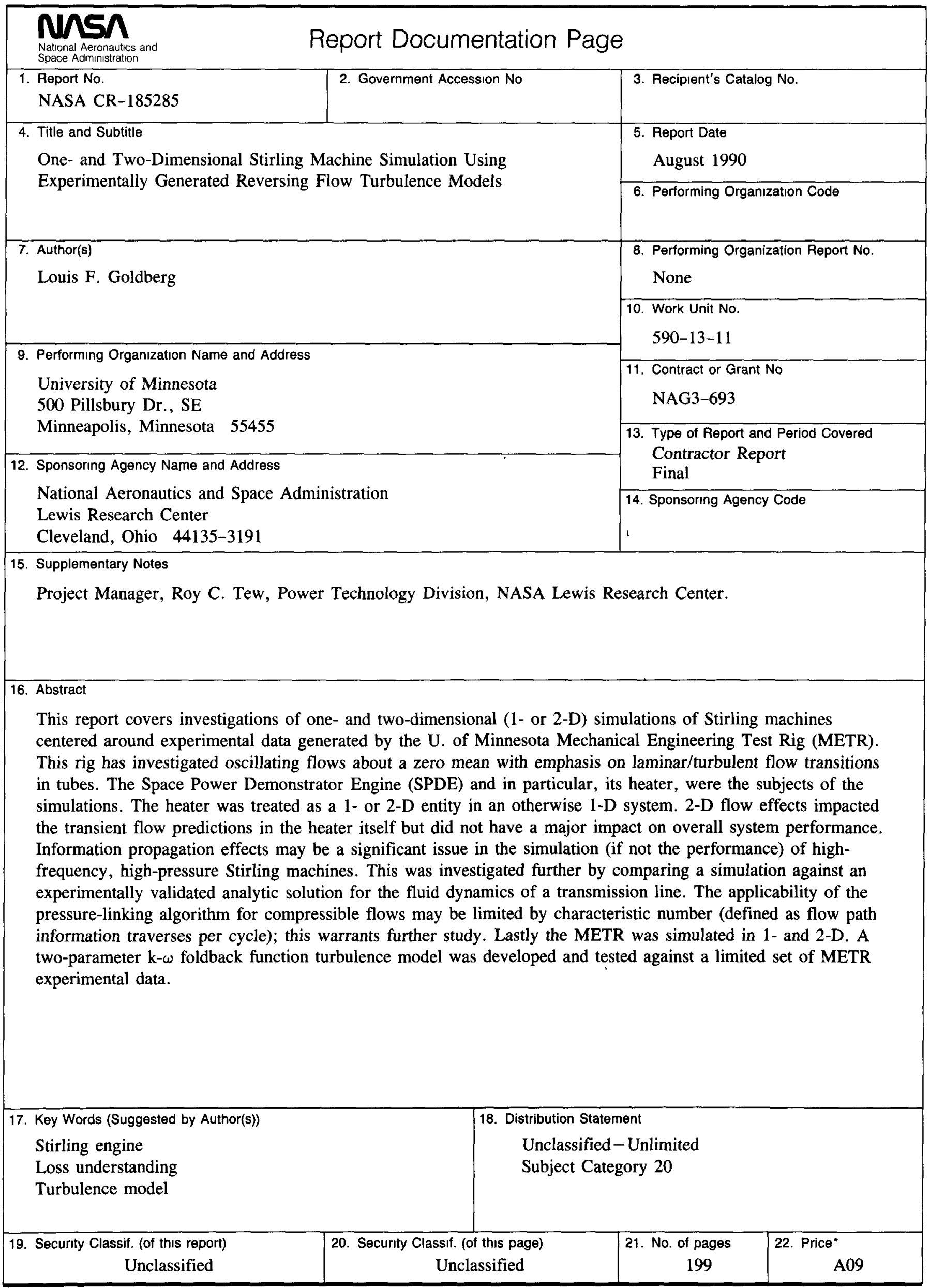


National Aeronautics and Space Administration

Lewls Research Center

Cleveland, Ohio 44135

Orfielel Budnese

Penaiky for Private Uee $\mathbf{s 0 0}$
FOURTH CLASS MAIL

ADDRESS CORRECTION REQUESTED

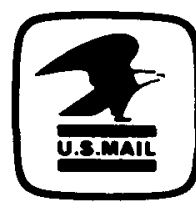

Postage and Fees Paid

Natıonal Aeronautics ano

Space Administration NASA 45 